\title{
PHYSICAL TESTING OF POTENTIAL FOOTBALL HELMET DESIGN ENHANCEMENTS
}

\author{
A Thesis \\ presented to \\ the Faculty of California Polytechnic State University, \\ San Luis Obispo
}

\author{
In Partial Fulfillment \\ of the Requirements for the Degree \\ Master of Science in Mechanical Engineering
}

by

Michael Jeremy Schuster

June 2016 
(C) 2016

Michael Jeremy Schuster

ALL RIGHTS RESERVED 


\section{COMMITTEE MEMBERSHIP}

TITLE:

Physical Testing of Potential Football Helmet Design Enhancements

AUTHOR:

Michael Jeremy Schuster

DATE SUBMITTED:

June 2016

COMMITTEE CHAIR:

Peter Schuster, Ph.D.

Professor of Mechanical Engineering

COMMITTEE MEMBER:

Brian Self, Ph.D.

Professor of Mechanical Engineering

COMMITTEE MEMBER:

Joseph Mello, Ph.D.

Professor of Mechanical Engineering 


\begin{abstract}
Physical Testing of Potential Football Helmet Design Enhancements

Michael Jeremy Schuster
\end{abstract}

Football is a much loved sport in the United States. Unfortunately, it is also hard on the players and puts them at very high risk of concussion. To combat this an inventor in Santa Barbara brought a new design to Cal Poly to be tested.

The design was tested in small scale first in order to make some preliminary conclusions about the design. In order to fully test the helmet design; however, full scale testing was required. In order to carry out this testing a drop tower was built based on National Operating Committee on Standards for Athletic Equipment, NOCSAE, specification. The drop tower designed for Cal Poly is a lower cost and highly portable version of the standard NOCSAE design. Using this drop tower and a 3D printed prototype the new design was tested in full scale.

With the results of the full scale testing regime and computer modeling done by another graduate student, it was concluded that the new design did not reduce a player's risk for concussion. The new design increased the SI value for the helmet by a factor of 2.5. 


\section{ACKNOWLEDGMENTS}

I would like to thank several people who without them my thesis would not have been possible. The main person who I would like to thank is Steven Warnert. Without his help throughout the process my testing and assembly could not have been finished. Additionally, I would like to thank George Rodriguez with his help with accomplishing my small scale testing. I would like to thank my committee chair, Dr. Schuster, and my committee members, Drs. Mello and Self, for their help and assistance throughout the

year. Their advice helped me through my thesis and the trials and tribulations which were involved.

Finally, and most importantly, I would like to thank my family and especially my girlfriend, Danielle. They have had to put up with me being incredibly busy the last year and have never held it against me.

Thank you to everyone! 


\section{TABLE OF CONTENTS}

Page

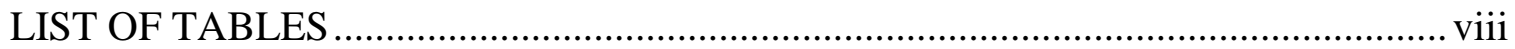

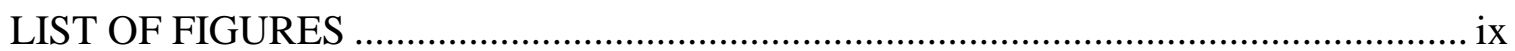

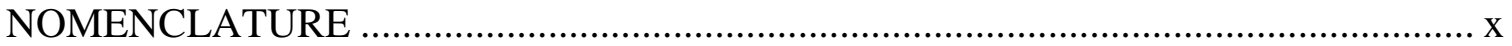

CHAPTER

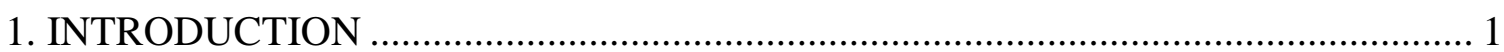

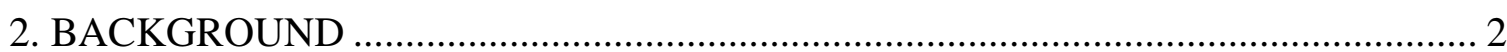

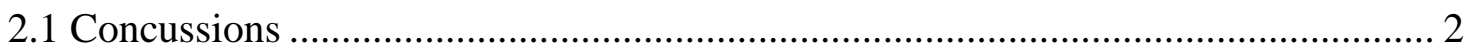

2.2 Current Helmet Designs................................................................................... 5

2.2.1 Football Helmets ...................................................................................... 6

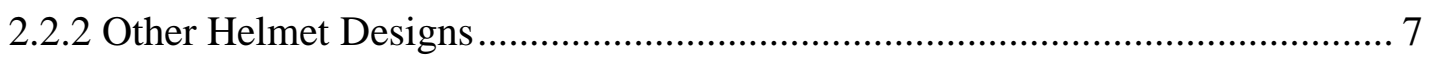

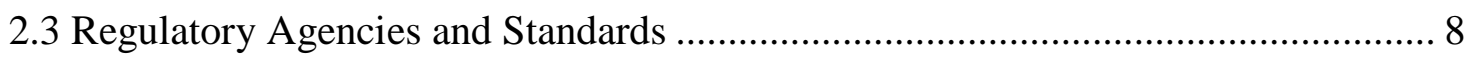

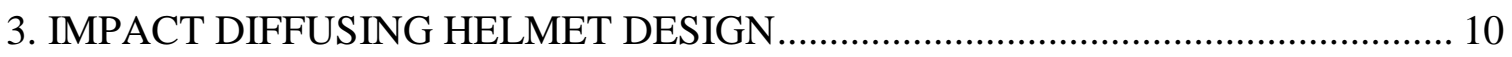

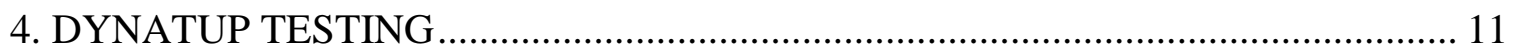

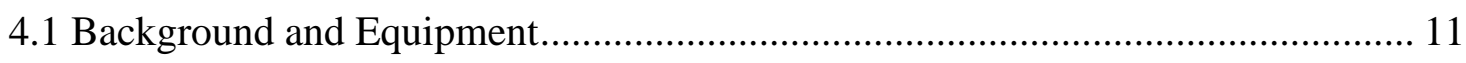

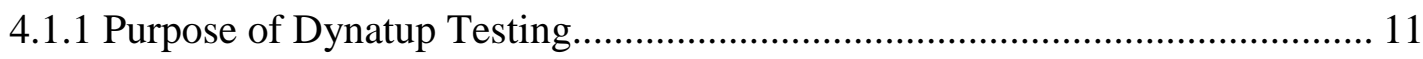

4.1.2 Dynatup Impact Tester........................................................................ 12

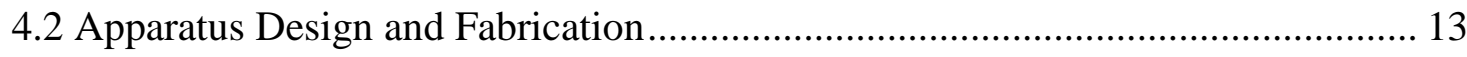

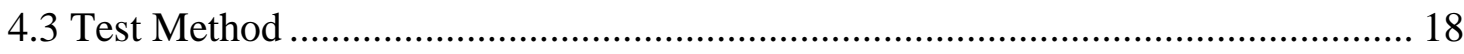

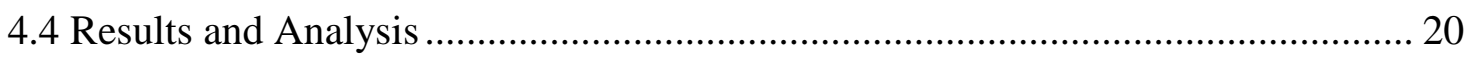

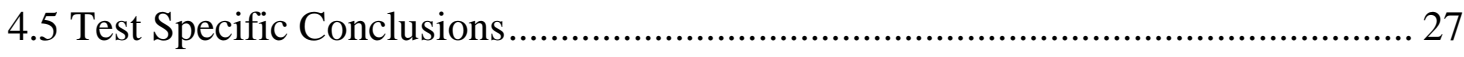

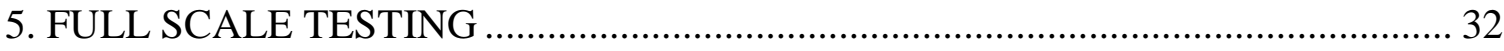

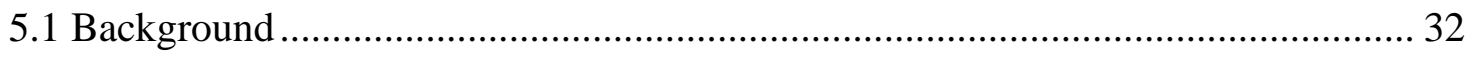

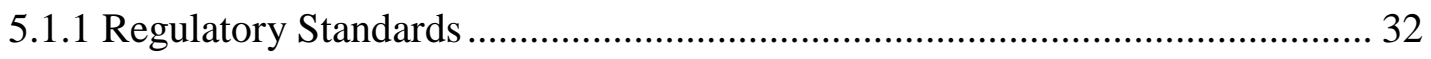

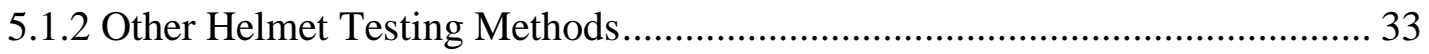

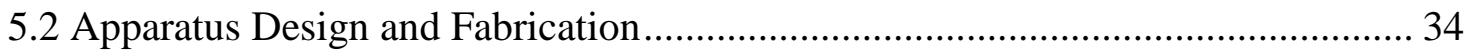

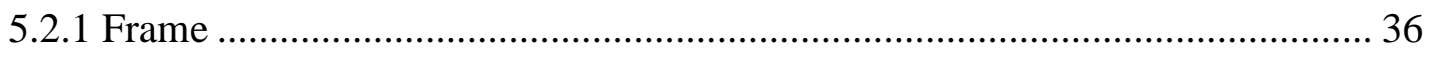

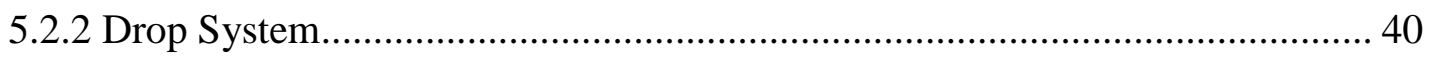

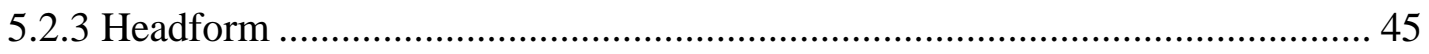

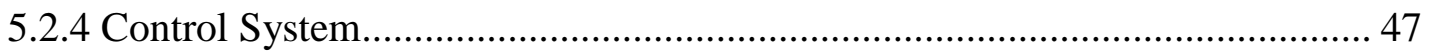

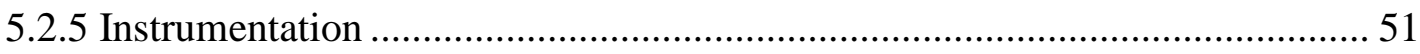

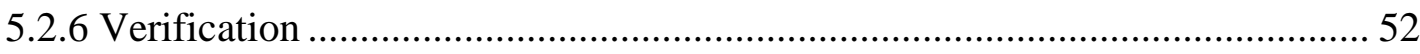

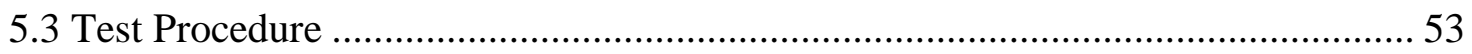




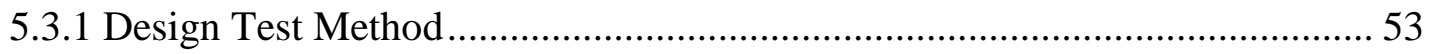

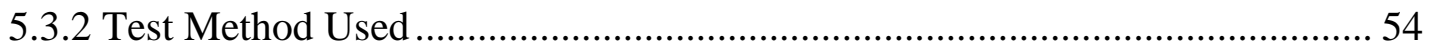

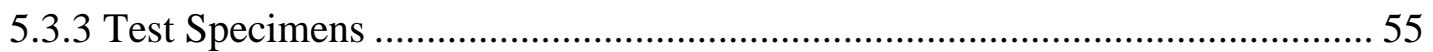

5.4 Drop Tower Observations, Discussion, and Future Work .................................. 58

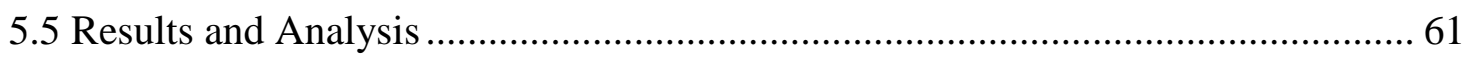

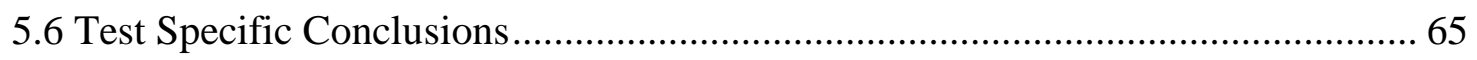

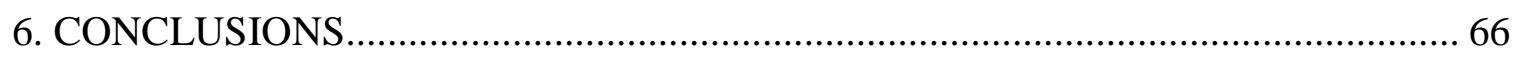

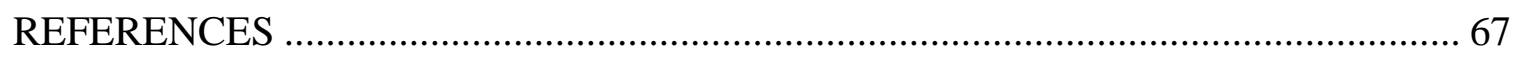

APPENDICES

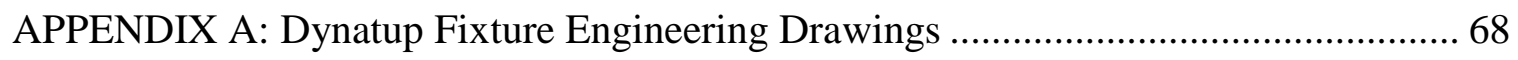

APPENDIX B: Small Scale Testing Analysis MATLAB Code.................................... 77

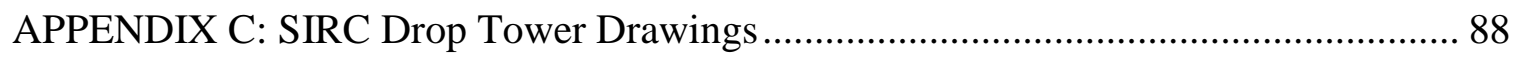

APPENDIX D: Helmet Drop Tower Engineering Drawing ....................................... 102

APPENDIX E: Full Scale Testing Analysis Code.................................................... 121

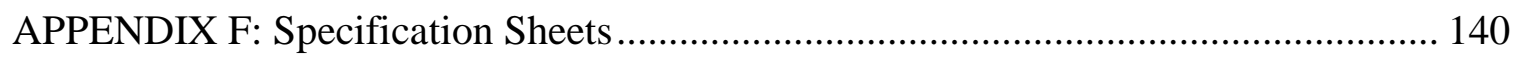

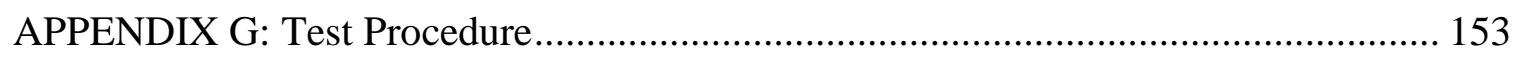

APPENDIX H: Axial Accelerations for Full Scale 2ft drop test................................. 156 


\section{LIST OF TABLES}

Table Page

Table 1. Small Scale test results from 1ft drop height for all three helmet types ............ 21

Table 2. Data from the small scale two foot drop height tests....................................... 25

Table 3. Drop test machine verification runs ............................................................... 53

Table 4. Drop tower tolerances for both test heights ................................................ 53

Table 5. Results from the full scale testing of the football helmet prototypes ................ 64 


\section{LIST OF FIGURES}

Figure Page

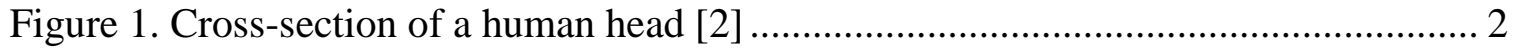

Figure 2. A common football helmet available today [12] ............................................ 5

Figure 3. The Xenith Epic Varsity football helmet....................................................... 7

Figure 4. An example of the marking a helmet receives when NOCSAE certified ...........9

Figure 5. The Dynatup 8250 that the Mechanical Engineering Departments owns ......... 12

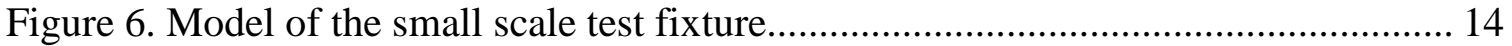

Figure 7. Dynatup test fixture base shown separate and installed in Dynatup ................. 17

Figure 8. Set up for a test run, sample attached is blurred due to the NDA ..................... 18

Figure 9. Boxplots for SI and EDC for the $1 \mathrm{ft}$ drop tests ........................................... 23

Figure 10. Raw, filtered, processed data from 1ft control, the processed first impulse ... 24

Figure 11. Boxplots for SI and EDC for 2ft drop heights.......................................... 26

Figure 12. Tukey comparison plots for $1 \mathrm{ft}$ drop height of SI and EDC ......................... 28

Figure 13. Tukey comparison plots for 2ft drop height for SI and EDC ......................... 29

Figure 14. NOCSAE specified drop tower design...................................................... 33

Figure 15. The NOCSAE style drop tower designed for Cal Poly .................................. 35

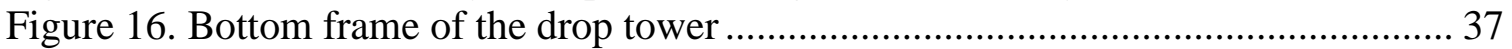

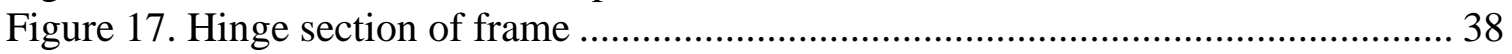

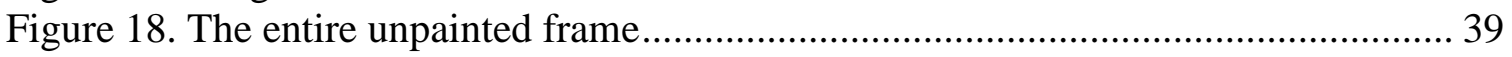

Figure 19. The cable rigging to connect the cable to the upper frame............................ 40

Figure 20. The lift bar showing the electromagnet, the limit switch, and the eyebolt...... 41

Figure 21. Harbor Freight winch responsible for positioning of lift bar ......................... 42

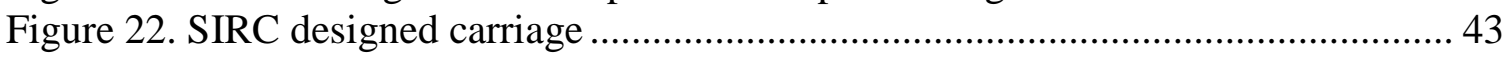

Figure 23. The safety bracket attached to the carriage ............................................... 44

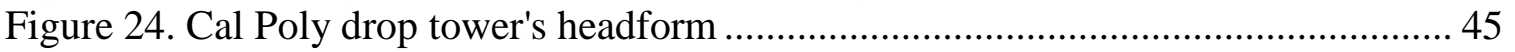

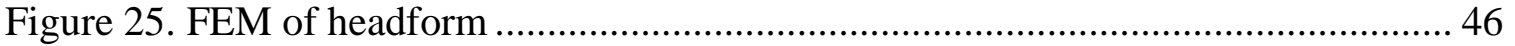

Figure 26. The outside and inside of the control system enclosure ................................. 47

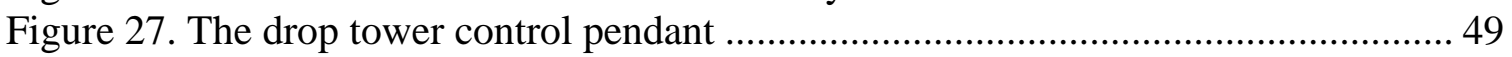

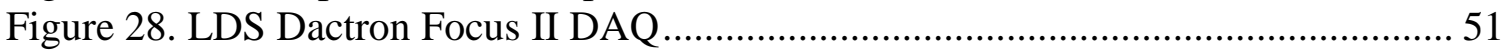

Figure 29. The PCB 482C05 Signal Conditioner ........................................................... 51

Figure 30. Photoscale for velocity measurement ..................................................... 52

Figure 31. FDM control shell outer surface and inner surface with padding installed..... 56

Figure 32. Control helmet after being destroyed by drop testing .................................. 57

Figure 33. The bent stem attached to the headform...................................................... 59

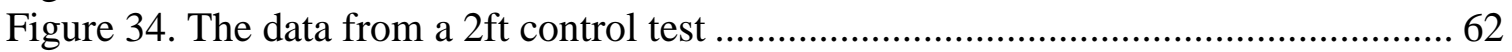

Figure 35. Single impulse (filtered) from full scale control test.................................... 63 


\section{NOMENCLATURE}

EDC - Energy Diffusion Coefficient

HIC - Head Impact Criterion

ISO - International Organization for Standardization

NOCSAE - National Operating Committee on Standards for Athletic Equipment

SAE - Society of Automotive Engineers

SI - Severity Index

SIRC - Southern Impact Research Center 


\section{INTRODUCTION}

In today's world the safety of athletes is very important. One of the most dangerous sports for players is American Football. In this sport the players run into each other at

full speed and sustain very energetic impacts. These impacts put the players at a great risk for concussions. This fact has been recently brought to mainstream attention by news articles and even a feature motion picture starring Will Smith. To combat this risk, the NFL, NCAA, and major manufacturers have been working of various designs to combat the risk of concussion. An inventor in Santa Barbara, California has also come up with a new design which he feels will be able to better reduce the risk of concussions. His design uses a new method of reducing the impact felt to the player's head. 


\section{BACKGROUND}

The purpose of this new football helmet design is to reduce the risk of concussion in football players. In college football, about 3,400 concussions were endured last year by athletes despite the use of current football helmets. Additionally, many of these players sustained more than one concussion in their athletic career. [1]

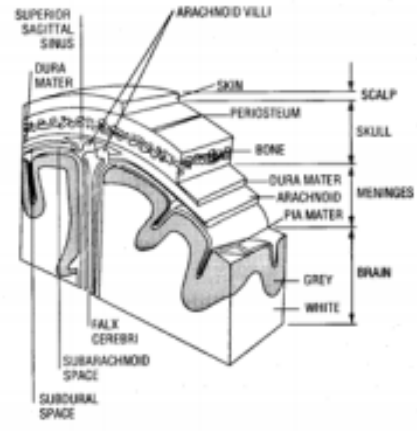

Figure 1. Cross-section of a human head [2]

\subsection{Concussions}

A concussion is a type of traumatic brain injury. Injuries to the brain are caused by a head impact or sudden head movement. Injuries can be caused by the skull fracturing and pushing against the brain. Additionally, injury can be caused by the brain impacting the interior of the skull or from the brain being stressed through shear, tension, or compression. [2] The anatomy of the human head can be seen in Figure 1.

Concussions can cause a variety of symptoms; from headaches to memory and cognitive problems to the condition Chronic Traumatic Encephalitis, CTE, which can cause death. If a head sustains another impact before the brain is fully able to recover, whether the 
second impact could have caused a concussion or not, the symptoms can exacerbate and cause even more damage to the brain. [1]

Not much is known about concussions and predicting the possibility of a concussion is a very complex issue. The risk of concussion is based off of both the magnitude and duration of the acceleration. This was first studied by researchers at Wayne State University. The curve they developed is called the Wayne State Tolerance Curve, or WSTC, which shows the tolerance level of humans. Another curve was developed based on WSTC, called the Gadd Severity Index or GSI. The combination of both of these techniques brought about the Head Injury Criterion, or HIC, developed by the NHTSA.

To compute HIC, the formula below is used

$$
H I C=\left[\frac{1}{t_{2}-t_{1}} \int_{t_{1}}^{t_{2}} a d t\right]^{2.5}\left(t_{2}-t_{1}\right)
$$

Where:

$\mathrm{a} \equiv$ resultant acceleration

$\mathrm{t}_{2}-\mathrm{t}_{1}$ is less than or equal to $36 \mathrm{~ms}$

$\mathrm{t}_{2}$ and $\mathrm{t}_{1}$ maximize HIC

Values of HIC above 1000 are considered to be above the safe limit. Later on, the maximum length of time was changed from 36 milliseconds to 15 milliseconds. [2] This criterion is what is regularly used in automotive testing and vehicle impacts for the automotive industry. A value of 1000 is the threshold of safety for general use of $\mathrm{HIC}_{36}$ and 700 for $\mathrm{HIC}_{15}$. 
Unlike the automotive industry the athletic industry is regulated to use Severity Index or SI. It is very similar to HIC. It is computed using

$$
S I=\int_{0}^{T} A^{2.5} d t
$$

Where:

$\mathrm{A} \equiv$ resultant acceleration

$\mathrm{T} \equiv$ time when acceleration drops below 4 g's

Time 0 is when acceleration rises above 4 g's

This criterion is what is used by NOCSAE, the main body which regulates football and other athletic helmets and protective gear. [3] If the SI value exceeds 1200 it means that the risk of concussion is extremely great and the helmet would be considered a failure.

Both of these criteria are based upon the work of Charles Gadd who first proposed the formulas consisting of the integral of the acceleration raised to a weighting factor be used to quantify concussion risk. This is based upon a curve fit of experimental data of probable concussions that were recorded when the skull of a cadaver fractured. [4]

Another approach which is sometimes used to quantify whether a concussion may occur is simple maximum linear acceleration. This method is used in standards published by ASTM. They specify the max acceleration the head can endure with limited concussion risk to be 300 g's. This method does not take into consideration the full effect of an impulse event since the length of time the impact takes place in is not taken into account. 


\subsection{Current Helmet Designs}

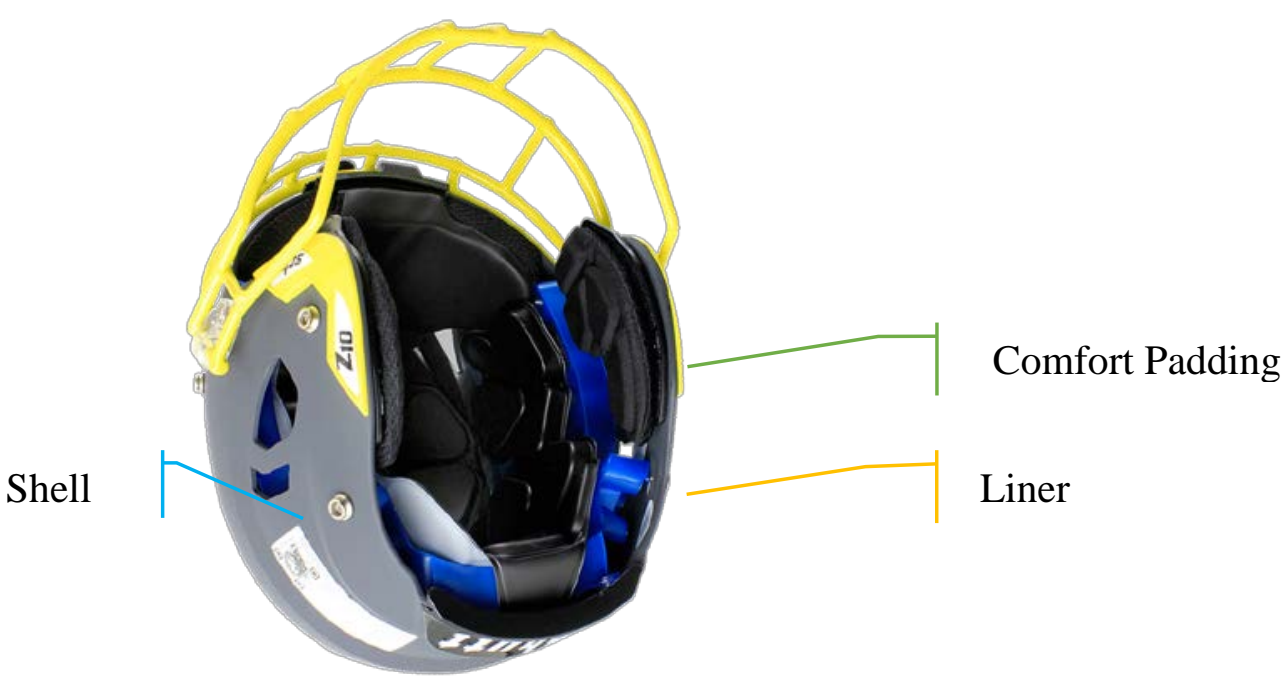

Figure 2. A common football helmet available today [12]

In this day and age there is a lot of research that is going into new helmet designs.

Helmets normally consist of four main parts: a rigid shell, a liner, a retention system, and a comfort padding. The rigid shell's main purpose is to prevent things from penetrating the helmet as well as to help distribute the force of an impact around the helmet. The liner's job is to absorb energy and prevent it from being transferred to the head, normally by crushing or other types of deformation. The retention system is required to keep the helmet positioned on the head correctly and to keep it on the head during an impact. Finally, the comfort padding is in place to allow the helmet to be worn for hours without pain to the wearer. [5] A good helmet can slow the acceleration felt by the head by as much as fifty percent. [1]

The purpose of a helmet is twofold: to spread the force of an impact to a greater surface area and to increase the duration of an impact. By increasing the duration of an impact, 
even if by a millisecond, the same amount of energy is transferred to the helmet but the impulse felt by the brain is reduced. This can turn what may be a hit that causes a concussion, or death, to one which is safe for a player. Like the common phrase, "it's not the fall that kills you, it's the sudden stop," it's the sudden stop which causes a concussion. Making the impact less sudden lessens the risk of concussion. [5]

\subsubsection{Football Helmets}

Football helmets have the same basic structure of any other helmet but are built to sustain multiple impacts without having to be replaced. A traditional football helmet has a shell made of polycarbonate or a similar polymer. This shell is used to hold together the rest of the helmet as well as display the team's colors and logo. The liner of a football helmet is where the largest difference exists between a typical helmet and one designed for football. The liners in football helmets are made to work multiple times. There are a variety of designs that manufacturers use. Some helmets utilize an egg-crate design made of plastic while others use foam which will not plastically deform. The retention system of a traditional football helmet is a chin strap. The chin strap uses quick release snaps to attach to the helmet. Like the name implies, the strap uses a chin cup which rests against the chin to keep the helmet in place. Comfort padding is another area where helmets manufacturers vary. Most will use a soft foam to provide comfort to the player's head. Used in both the comfort and retention systems of many helmets are inflatable air bladders. These bladders have two purposes: to keep the helmet in contact with the head, its job in the retention system, and to perfect the fit of the helmet, its job in the comfort system. 
The current top performing helmet, rated by the NFL, is the Xenith Epic Varsity, as shown in Figure 3. [6] This helmet uses an air filled shock absorber system as a liner.

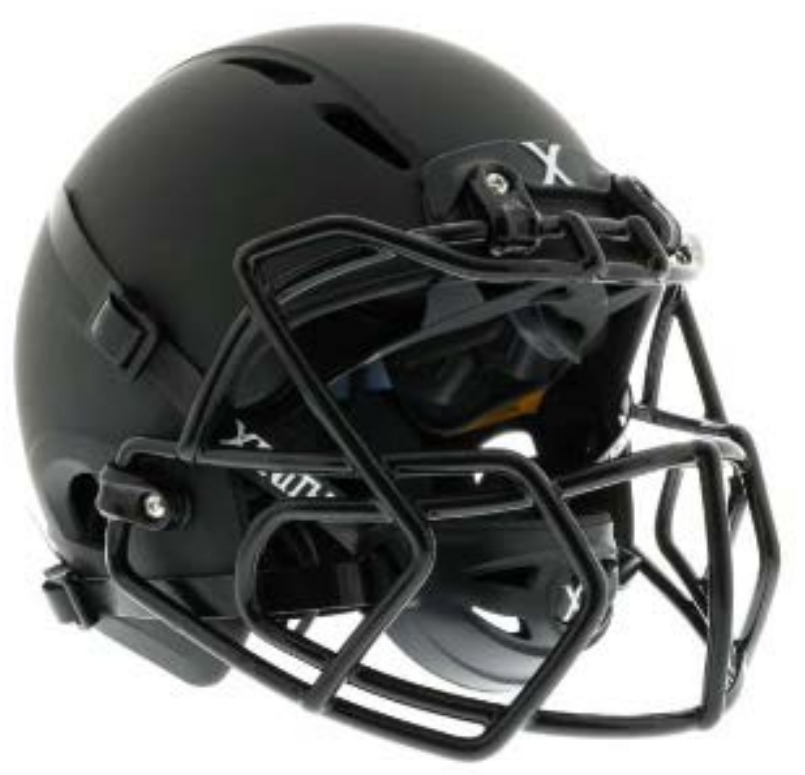

Figure 3. The Xenith Epic Varsity football helmet

A new helmet which is getting a lot of press is the VICIS Zero1 Impact Reduction helmet. It is a combination helmet which uses both a newly designed liner system and shell they call the LODE shell. This shell locally deforms, just like a car bumper does. This helmet design has won awards from the NFL for its unique and superior features. [7]

\subsubsection{Other Helmet Designs}

A traditional helmet, one not used in football, is designed to be used only once. Because of this, typical helmets such as motorcycle or bicycle helmets, can more effectively reduce concussion risk. The liner of a motorcycle helmet tends to be made out of a foam material, such as expanded polystyrene. This material crushes or fractures to dissipate 
the impact energy. [5] Since these helmets can only take a single impact they are not useful for football, where a single player can experience many impacts even in one game.

\subsection{Regulatory Agencies and Standards}

With the safety of the many athletes in the United States who play football, it is understandable that many agencies produce regulations to make sure protection equipment is adequate. The main agency for the safety of non-professional football players is the National Operating Committee on Standards for Athletic Equipment, or NOCSAE (pronounced “Nock-See”). However, many other agencies publish standards that are relevant to football helmets and their testing. ASTM International also publishes standards that describe the testing and design requirements for football helmets.

Standards published by the Society of Automotive Engineers, or SAE, are also used since impact testing of football helmets is similar to impact testing of crash test dummies in cars.

For NOCSAE there are two standards that are most noteworthy for testing of football helmet designs, they are NOCSAE Documents 001 and 002. 


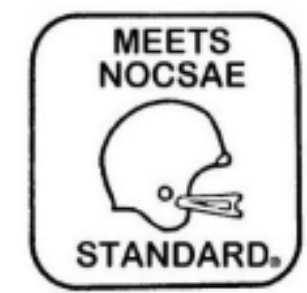

Figure 4. An

example of the

marking a helmet

receives when

NOCSAE certified

Document 001 describes the test methods for testing headgear. This document describes the test methods as well as the apparatus used to test football and other sports helmets. Document 002 lists the performance specification required for newly manufactured football helmets. It lists things such as the speeds and temperatures a football helmet must be tested at to be certified. If a helmet meets these specifications it receives a marking similar to Figure 4. Another NOCSAE specification of note is Document 004, which lists the specifications for football helmets which are being recertified.

Recertification has lower requirements, since the helmet already passed the more stringent newly manufactured specifications.

ASTM also has two standards relating to football helmets. ASTM F429 describes the test method to determine the shock-attenuation characteristics of football helmets and F717 lists the specifications for football helmets. The SAE standard used in football helmet testing is J211-1. This standard describes the electronic instrumentation used for impact tests. It describes methods to determine required sampling frequencies and ranges needed for certain impacts. 


\section{IMPACT DIFFUSING HELMET DESIGN}

There is a new helmet design that is being developed by an inventor. This design's goal is to reduce the risk of concussion in football players from youth to professional. This new design for a football helmet’s inventor came up with this idea while watching a game on television. The inventor is Brad Bartholomay, from Santa Barbara, California. Mr. Bartholomay brought his idea for this helmet to Cal Poly professors Peter Schuster and Brian Self to discuss the helmet design and these two professors are now on the committee for this thesis.

Bartholomay’s design attempts to lessen the impact felt by the player. The design uses features added to the helmet that try to add to the damping effect of the helmet. There are two experimental samples of this helmet, Experimental Design 1 and 2. These two helmets will be referred to as ED1 and ED2. ED2 is a modification of ED1 which is a modification of a standard, control helmet. Unfortunately, the exact features of the helmet designs that may decrease the concussion risk are proprietary and covered by a non-disclosure agreement. Because of this the designs will not be explained and referred to only by their names. 


\section{DYNATUP TESTING}

The Dynatup test apparatus is the only impact test apparatus owned by the Cal Poly Mechanical Engineering department. It was decided that this apparatus would be used for the testing of Bartholomay’s design because impact tests are more representative of the stresses undergone by helmets during a game of football than tests previously run by Mr. Bartholomay.

\subsection{Background and Equipment}

\subsubsection{Purpose of Dynatup Testing}

Dynatup testing was conducted so that small scale testing of the helmet designs could be done. The designer of the helmets had a three dimensional model made by additive manufacturing that was previously used by a Senior Project Team in Cal Poly's Industrial and Manufacturing Engineering Department. The testing done by the senior project team; however, did not simulate an impact so a new testing program was desired. Testing these small scale prototypes allowed a quicker turnaround for preliminary results without having to wait for full scale models and a full scale test apparatus to be built. 


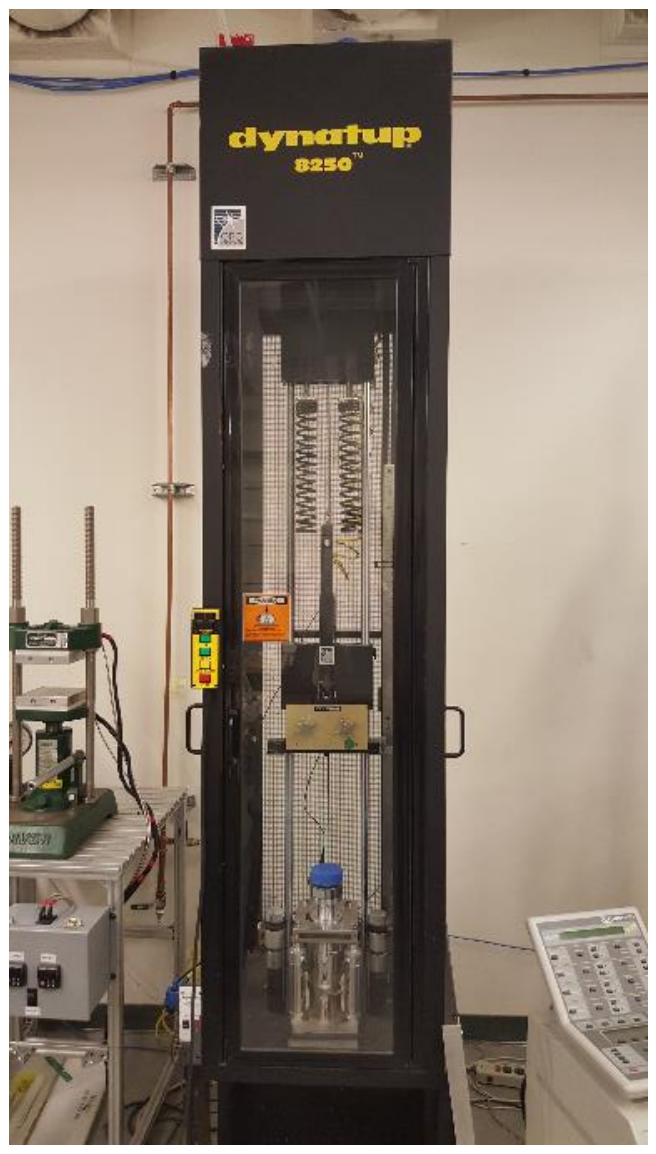

Figure 5. The Dynatup 8250 that the Mechanical Engineering

Departments owns

\subsubsection{Dynatup Impact Tester}

The California Polytechnic State University’s Mechanical Engineering Departments owns an impact test machine called a Dynatup 8250, it can be seen in Figure 5. The machine has a crosshead which is released and accelerated downwards towards a sample by gravity or through a pneumatic cylinder. The machine that the department possesses also has a pneumatically actuated clamp. The clamp uses four pistons to pull a moving plate down which clamps against a stationary plate. The crosshead is able to have a variable weight attached to it to increase or decrease the energy involved in impact. This machine was the impact tester used in the small scale testing for the football helmet 
prototypes. The crosshead was weighted with a 5.24lbf weight. This weight was chosen because it is the lightest, non-specialty weight so was the safest to use. The Dynatup machine normally has a Tup, a small rod shaped metal impactor with or without a load cell, attached to the crosshead. The tup was replaced for this testing with a specifically designed impactor which carried both the model prototypes, padding from a football helmet, and an accelerometer which was used to measure the acceleration versus time data to be later converted to severity index. The impactor weighed two pounds, bringing the falling weight to 7.24lbf. Instead of the normal sample which would be placed in the pneumatic clamp, an impact surface was placed.

\subsection{Apparatus Design and Fabrication}

In order to utilize the Dynatup for testing the helmet designs a fixture had to be created to modify the operation of the test apparatus. The fixture was designed in Solidworks and analyzed using hand calculations for the strength of the stem. The fixture was made of two parts, the impactor assembly and the base. The drawings for this fixture are included in APPENDIX A: Dynatup Fixture Engineering Drawings and the fixture can be seen in Figure 6. 


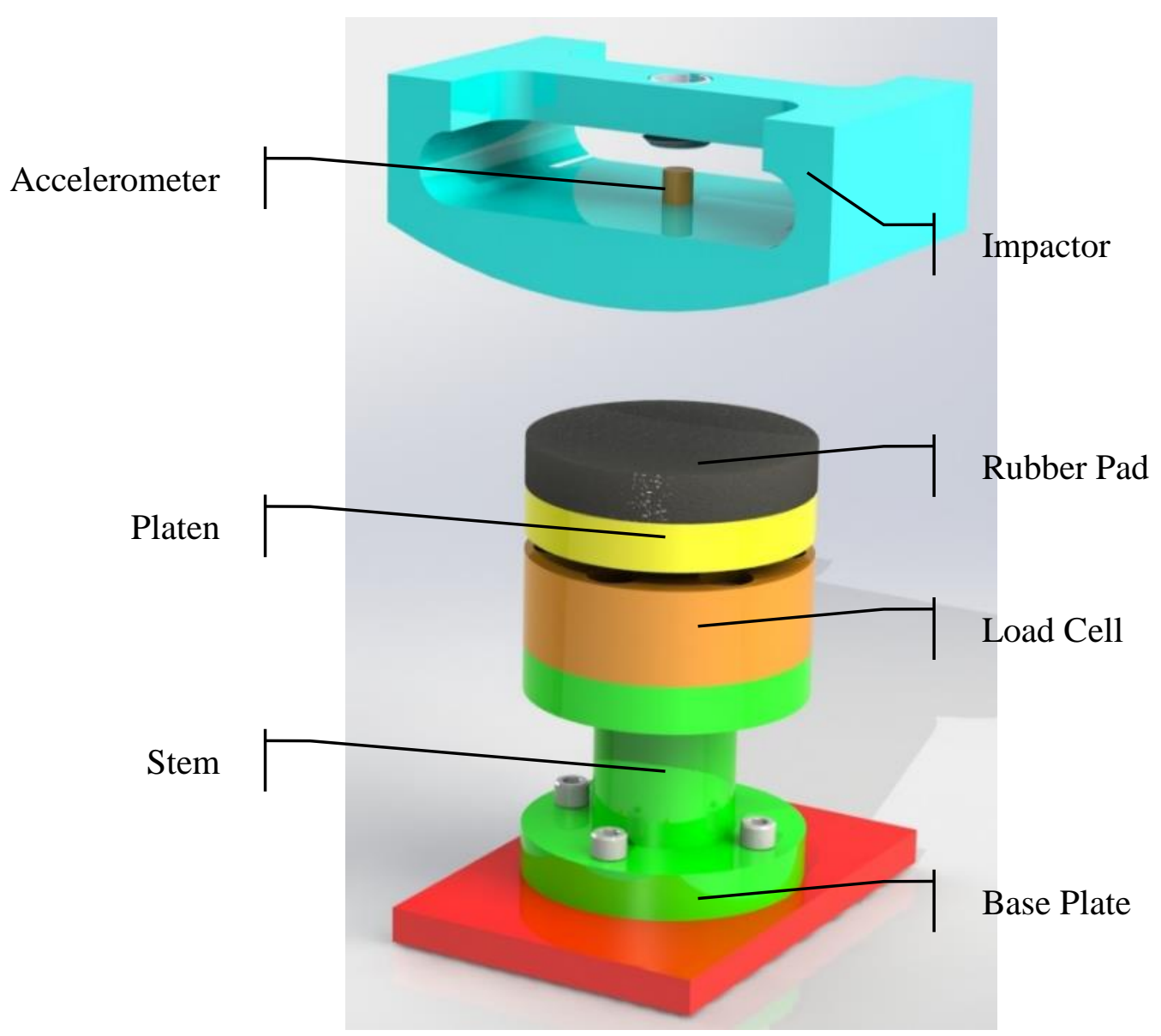

Figure 6. Model of the small scale test fixture

The blue part in Figure 6 is the impactor. It holds the model of the experimental and control helmets. The impactor also holds an accelerometer which is used to quantify the impact. The impactor is held to the crosshead of the Dynatup with the same bolt which is normally used to hold tups onto the machine. The impactor was made of Aluminum 2024 and machined using a CNC mill in the Cal Poly Mustang '60 machine shop.

The other parts in the model make up the base. The purpose of this subassembly is to provide a surface for the impactor to hit. The surface which the impact occurs on is shown as the black piece in Figure 6. The black piece is a piece of Buna-N Rubber with a durometer of 40A. This durometer is specified by NOCSAE to be $38 \mathrm{~A} \pm 5$ [3]. The 
specification is for a specific rubber pad called an MEP pad, however these pads are very expensive so the Buna- $\mathrm{N}$ pad was substituted. Buna- $\mathrm{N}$ was chosen because it was the only rubber available with the correct durometer rating. This pad is attached with adhesive to the platen, yellow in the figure. This platen is used to transmit the force of the impact to a load cell, shown in orange. The platen was machined on a lathe out of Aluminum 7075. The load cell used in this apparatus was an Omega LCH-1K 1000lbf load cell. The load cell is supported by the stem shown in green. This was also machined out of 7075 Aluminum. The stem was analyzed to ensure that it would not fracture or yield under repeated impacts. The stem was analyzed because it has the smallest diameter and the stepped shaft makes it susceptible to stress concentrations.

The first analysis carried out on the stem was an impulse momentum calculation to determine the force that the falling impactor will impart on it. Using the mass of the impactor and the speed at which it falls, $17.94 \mathrm{ft} / \mathrm{sec}$ [8], the force can be determined to be $852 \mathrm{lbf}$. This is calculated using an estimated impact time of one millisecond. The stress in the stem was then calculated to be 3736 psi. This is with the stress concentration factor of the flat bottom groove. Without the concentration factor the stress is only 482 psi. The final step in the calculations was to do fatigue calculations. This determined that for 7075 aluminum the stem is good for over 1000 impacts with a factor of safety of seven. 
The stem is held in place by the base plate, in red. The pneumatic clamp of the Dynatup clamps down on the base plate to hold the base of the test fixture in place. The completed base fixture is shown in Figure 7. 

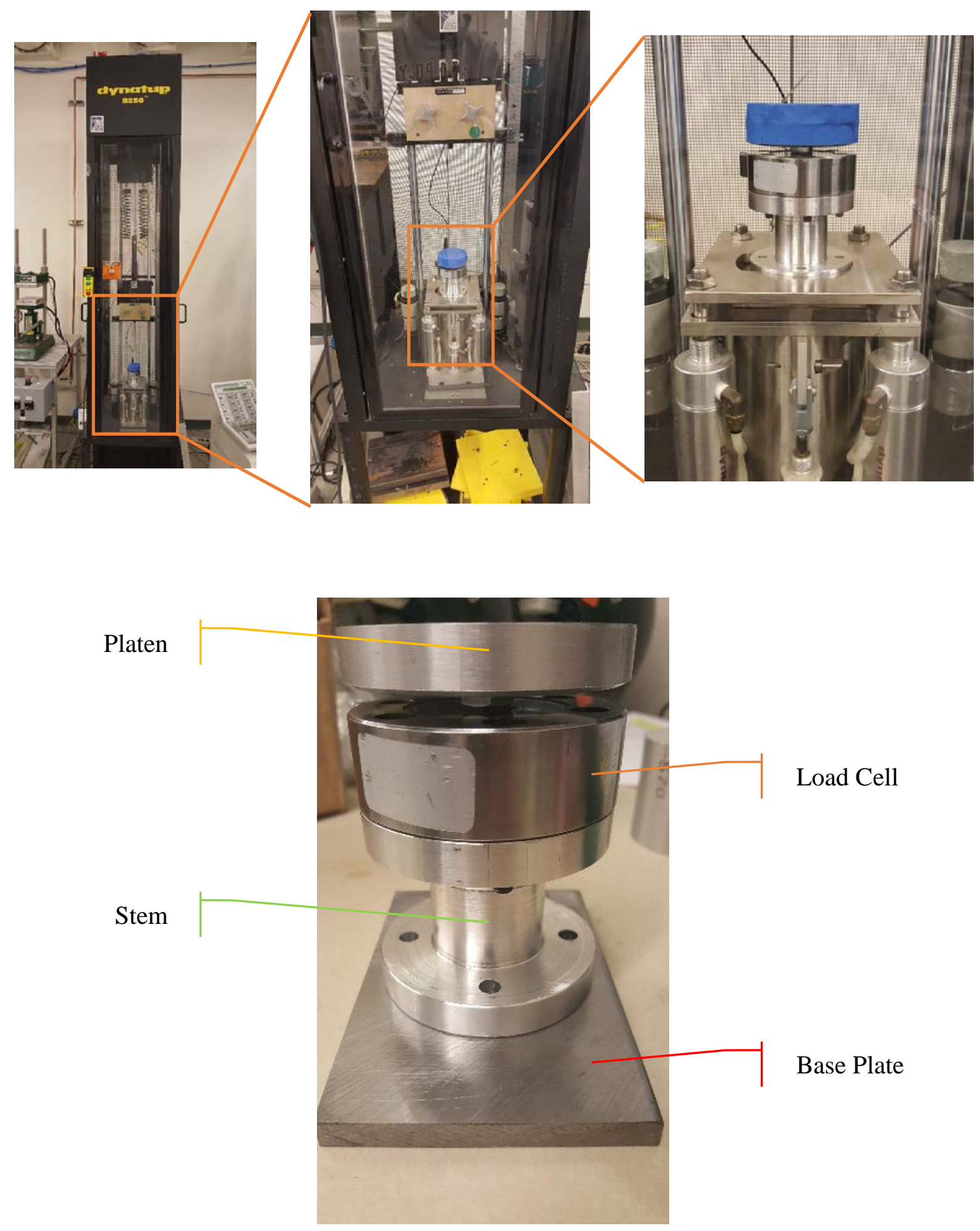

Figure 7. Dynatup test fixture base shown separate and installed in Dynatup 


\subsection{Test Method}

The test fixture which was created for the Dynatup was placed in the machine as shown in Figure 8. The accelerometer used for this test was a PCB 353B15. This is an ICP type accelerometer with a rating of 500g's. The accelerometer was attached to a signal conditioner to allow the data from accelerometer to be measured by the data acquisition system. The DAQ used was an LDS Dactron Focus II. It is normally used as a signal analyzer, but for this testing was merely used as a recording device. The sampling frequency used for this impact event was $16384 \mathrm{~Hz}$. This frequency was picked using the

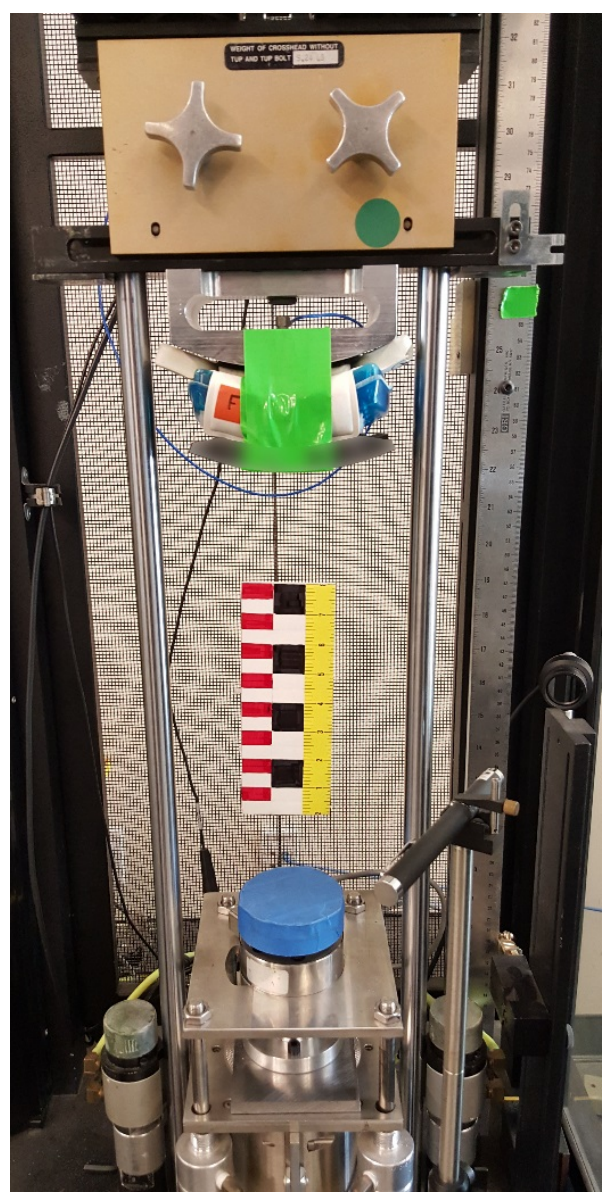

Figure 8. Set up for a test run, sample attached is blurred due to the NDA 
charts and tables that are presented by SAE in their specification relating to impact test instrumentation. [9]

The experimental samples; ED1, ED2, and the control; were attached to the impactor with standard padding from a Schutt football helmet sandwiched in between the impactor and the sample. The experimental samples were held to the impactor with duct tape. There were six sets of experimental conditions that were tested in the small scale testing phase. Each of the samples was dropped from two heights, a one foot drop and a two foot drop, to enable different drop speeds to be tested. For each of these different condition sets, ten test runs were conducted to ensure a statistical sample.

The initial test plan included using the load cell to corroborate the accelerometer data. This, however; was not possible due to the load cell readout possessed by the Mechanical Engineering department at the time of testing was inoperable. This led to the load cell not being used for the small scale tests.

The data that was recorded for each test run was the acceleration measured by the accelerometer. This was then processed to determine the Severity Index for each run. Additionally, for the first three test runs of each experimental condition the impacts were recorded with a high speed camera recording at 5000 frames per second. Using a scale in the background, the speed of the drops could be calculated by determining the number of frames that the impactor took to drop one inch. 
MATLAB was used to analyze the data once the tests were complete. Two MATLAB scripts were written, one to analyze the SI value of a single test and one to analyze the SI of multiple test runs to find an average SI. The scripts can be found in APPENDIX B: Small Scale Testing Analysis MATLAB Code.

\subsection{Results and Analysis}

The tests were carried out over the course of two days, with all of one height of test done in one day without adjustment. The mean SI was analyzed for ED1 and ED2 at both drop heights and then compared to the control's drop tests.

In order to help analyze the results, a new value was used. The value used is the Energy Dispersion Coefficient, or EDC. The EDC is a value not used by other researchers and was created for this research. This value is the ratio of the second to first impulse, shown as a percentage. The EDC was used as an alternative to the Coefficient of Restitution, known as COR. Originally, COR was to be used but its calculation was unreliable due to the difficulty in determining the arrival and departure speeds of the helmet from the impact pad. Alternatively, EDC is easily calculated from the accelerometer data and is calculated using the same MATLAB scripts used to calculate SI.

The data were refined first by a 4 pole Butterworth low pass filter with a cutoff frequency of $1000 \mathrm{~Hz}$, as required by NOCSAE, and then processed by setting any value of acceleration that is less than $4 \mathrm{~g}$ 's to zero. This is because the calculation of SI is only triggered once the acceleration passes above 4 g's and is ended when it drops back below 
4. Once the data is processed, the MATLAB code identifies the first impulse of the data and determines the SI from that. It then finds the second peak and determines the EDC from both the first and second impulses.

Table 1. Small Scale test results from 1ft drop height for all three helmet types

\begin{tabular}{|c|c|c|c|c|c|c|c|c|}
\hline \multicolumn{3}{|c|}{ Control from $1 \mathrm{ft}$} & \multicolumn{3}{|c|}{ Experimental Design 1 from $1 \mathrm{ft}$} & \multicolumn{3}{|c|}{ Experimental Design 2 from $1 \mathrm{ft}$} \\
\hline Run & SI & EDC & Run & SI & EDC & Run & $\mathrm{SI}$ & EDC \\
\hline 1 & 161.29 & 41.87 & 1 & 135.00 & 42.50 & 1 & 135.22 & 42.01 \\
\hline 2 & 164.04 & 42.33 & 2 & 134.99 & 42.77 & 2 & 136.03 & 42.74 \\
\hline 3 & 166.53 & 42.10 & 3 & 135.07 & 42.30 & 3 & 136.97 & 42.84 \\
\hline 4 & 167.04 & 42.41 & 4 & 133.62 & 42.96 & 4 & 136.74 & 42.39 \\
\hline 5 & 165.57 & 42.75 & 5 & 134.69 & 42.65 & 5 & 134.32 & 42.46 \\
\hline 6 & 161.42 & 42.18 & 6 & 135.30 & 41.70 & 6 & 135.99 & 42.16 \\
\hline 7 & 162.04 & 41.25 & 7 & 137.01 & 42.31 & 7 & 136.73 & 42.36 \\
\hline 8 & 165.27 & 42.23 & 8 & 133.21 & 42.06 & 8 & 134.54 & 42.36 \\
\hline 9 & 167.19 & 42.33 & 9 & 134.84 & 42.60 & 9 & 135.74 & 42.50 \\
\hline 10 & 164.67 & 42.00 & 10 & 135.33 & 42.07 & 10 & 134.47 & 42.75 \\
\hline 11 & 166.02 & 42.12 & 11 & 132.73 & 42.94 & & & \\
\hline 12 & 165.93 & 42.29 & 12 & 134.67 & 43.09 & & & \\
\hline 13 & 164.93 & 42.57 & 13 & 134.58 & 42.82 & & & \\
\hline & & & 14 & 135.19 & 43.12 & & & \\
\hline & & & 15 & 134.69 & 42.94 & & & \\
\hline MEAN & 164.76 & 42.19 & & 134.73 & 42.59 & & 135.68 & 42.46 \\
\hline DEVIATION & 2.03 & 0.36 & & 0.96 & 0.41 & & 1.00 & 0.26 \\
\hline
\end{tabular}

The one-foot drop results are shown in Table 1 and represented as a boxplot in Figure 9.

For the control and ED1 samples extra runs were conducted.

The data, first impulse, and EDC calculation are all plotted by the MATLAB script. The data from one of the 1ft drop height control test is shown in Figure 10. It can be seen from the plot of the data that this test has 6 discernable impact events, each peak coinciding with the helmet bouncing on the impact plate. It is also seen that the filtering of the data reduces the peak acceleration seen by the helmet specimen. This reduction in peak value is why the filter used for the data is standardized. From the plot of the single impulse it is observed that the control impact event shown had an impulse time of about 
13 milliseconds and a peak acceleration of about 75 g's, this is consistent with the other control drops from $1 \mathrm{ft}$. 


\section{Boxplot of SI}

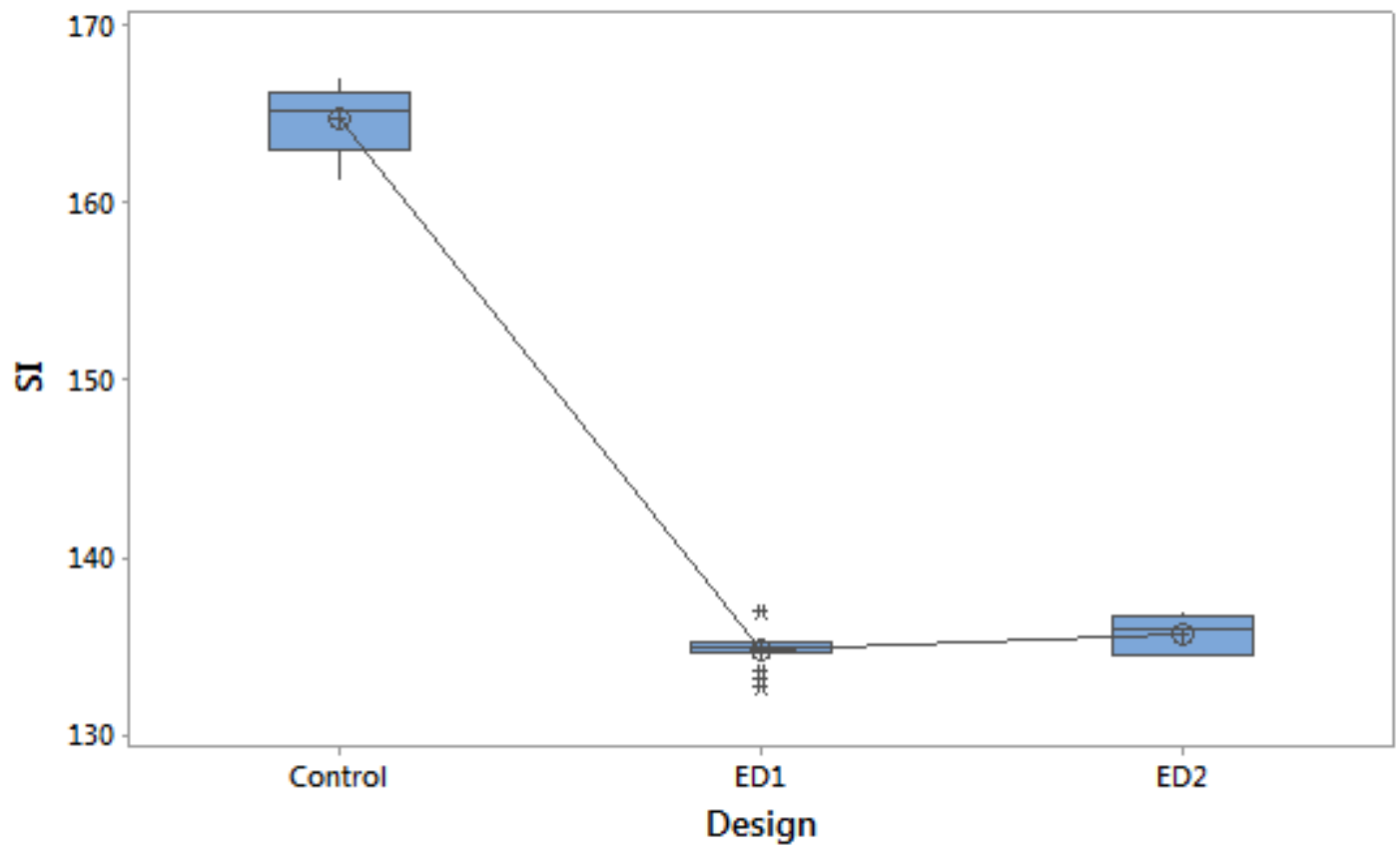

Boxplot of EDC

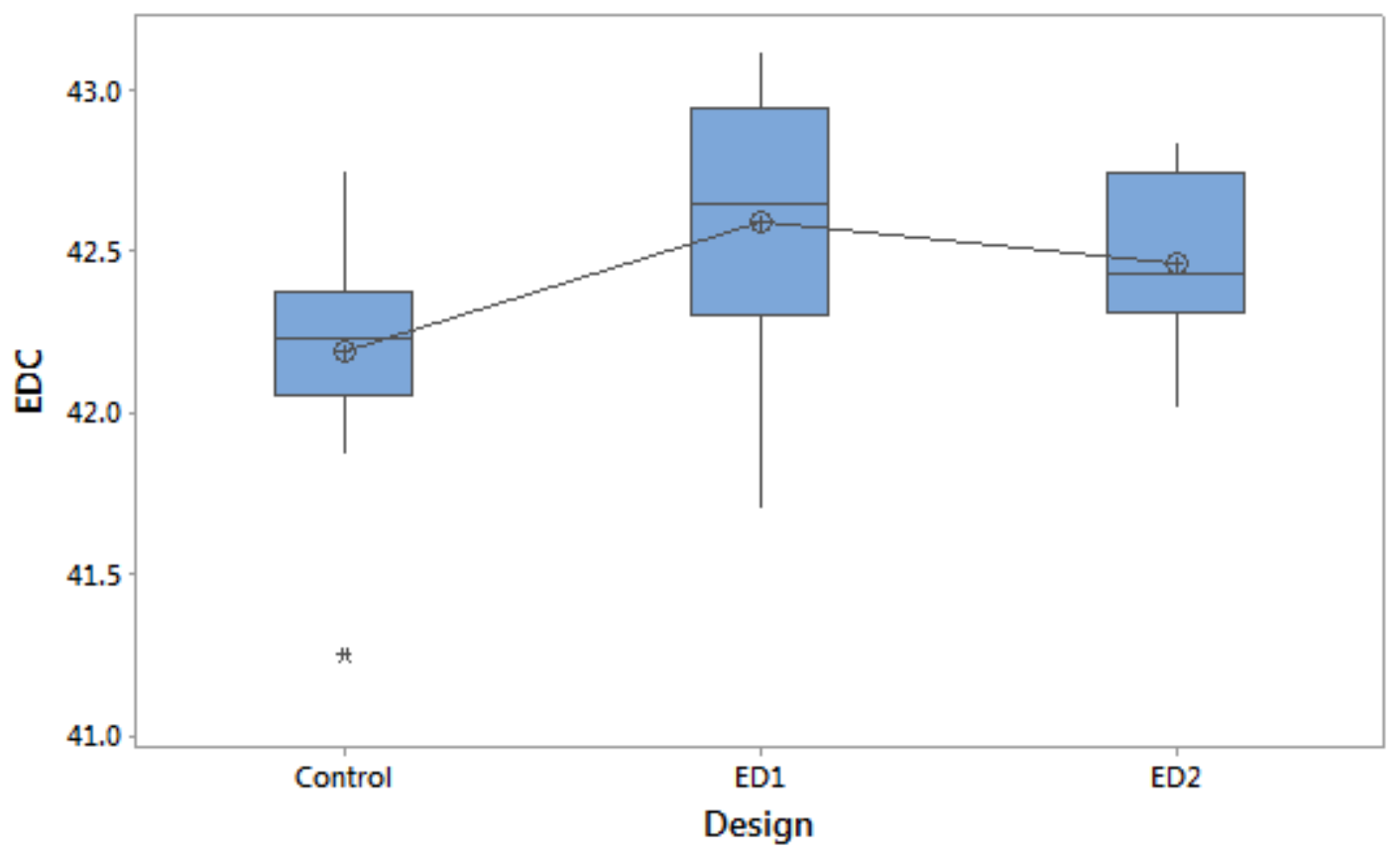

Figure 9. Boxplots for SI and EDC for the $1 \mathrm{ft}$ drop tests 

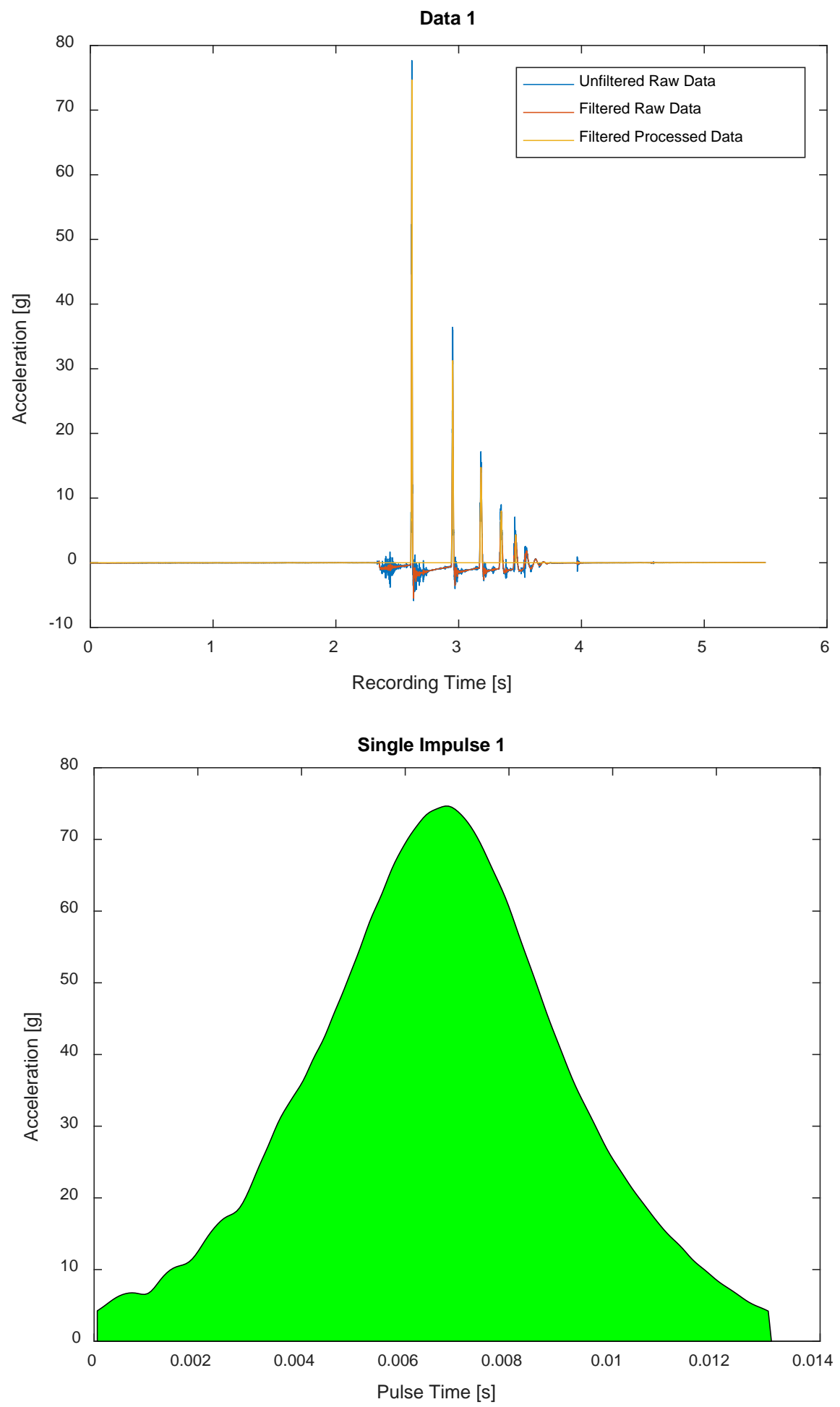

Figure 10. Raw, filtered, processed data from $1 \mathrm{ft}$ control, the processed first impulse 
Table 2. Data from the small scale two foot drop height tests

\begin{tabular}{|c|c|c|c|c|c|c|c|c|}
\hline \multicolumn{3}{|c|}{ Control from $2 \mathrm{ft}$} & \multicolumn{3}{|c|}{ Experimental Design 1 from $2 \mathrm{ft}$} & \multicolumn{3}{|c|}{ Experimental Design 2 from $2 \mathrm{ft}$} \\
\hline Run & $\mathrm{SI}$ & EDC & Run & SI & EDC & Run & $\mathrm{SI}$ & EDC \\
\hline 1 & 446.66 & 49.92 & 1 & 408.89 & 37.09 & 1 & 405.33 & 37.86 \\
\hline 2 & 447.22 & 49.46 & 2 & 412.06 & 37.21 & 3 & 418.30 & 38.47 \\
\hline 3 & 445.72 & 50.36 & 3 & 412.52 & 36.44 & 4 & 411.99 & 37.68 \\
\hline 4 & 447.00 & 50.31 & 5 & 407.47 & 37.20 & 5 & 413.05 & 38.07 \\
\hline 5 & 447.88 & 50.69 & 6 & 413.66 & 36.86 & 6 & 416.54 & 38.25 \\
\hline 6 & 446.38 & 50.44 & 9 & 409.92 & 38.50 & 7 & 413.44 & 36.52 \\
\hline 7 & 446.69 & 50.11 & 10 & 414.34 & 37.64 & 8 & 412.00 & 37.19 \\
\hline 8 & 446.83 & 49.80 & & & & 9 & 412.04 & 38.31 \\
\hline 9 & 446.83 & 50.27 & & & & 10 & 413.67 & 36.76 \\
\hline 10 & 446.54 & 50.28 & & & & & & \\
\hline MEAN & 446.77 & 50.16 & & 411.26 & 37.28 & & 412.93 & 37.68 \\
\hline DEVIATION & 0.56 & 0.35 & & 2.56 & 0.65 & & 3.59 & 0.70 \\
\hline
\end{tabular}

The two foot drop data is shown in Table 2 and represented as a boxplot in Figure 11.

The raw data and impulses are similar to the 1 foot drops. The differences are that the 2 foot data has a higher peak as well as a longer impact time, both leading to a higher calculated SI. There are some tests runs missing from the tables due to errors in triggering the DAQ and the first impulse not being recorded correctly. 


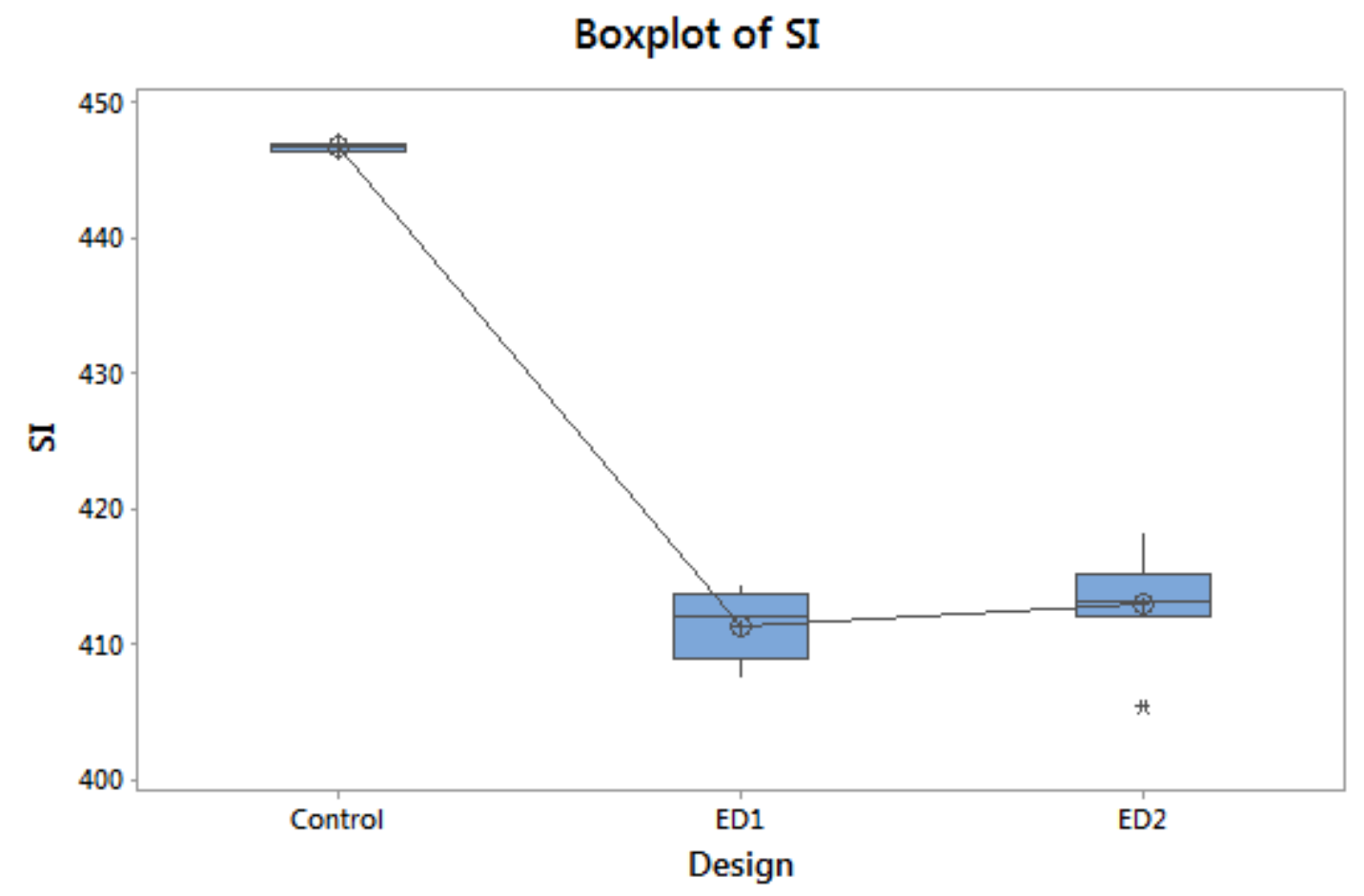

Boxplot of EDC

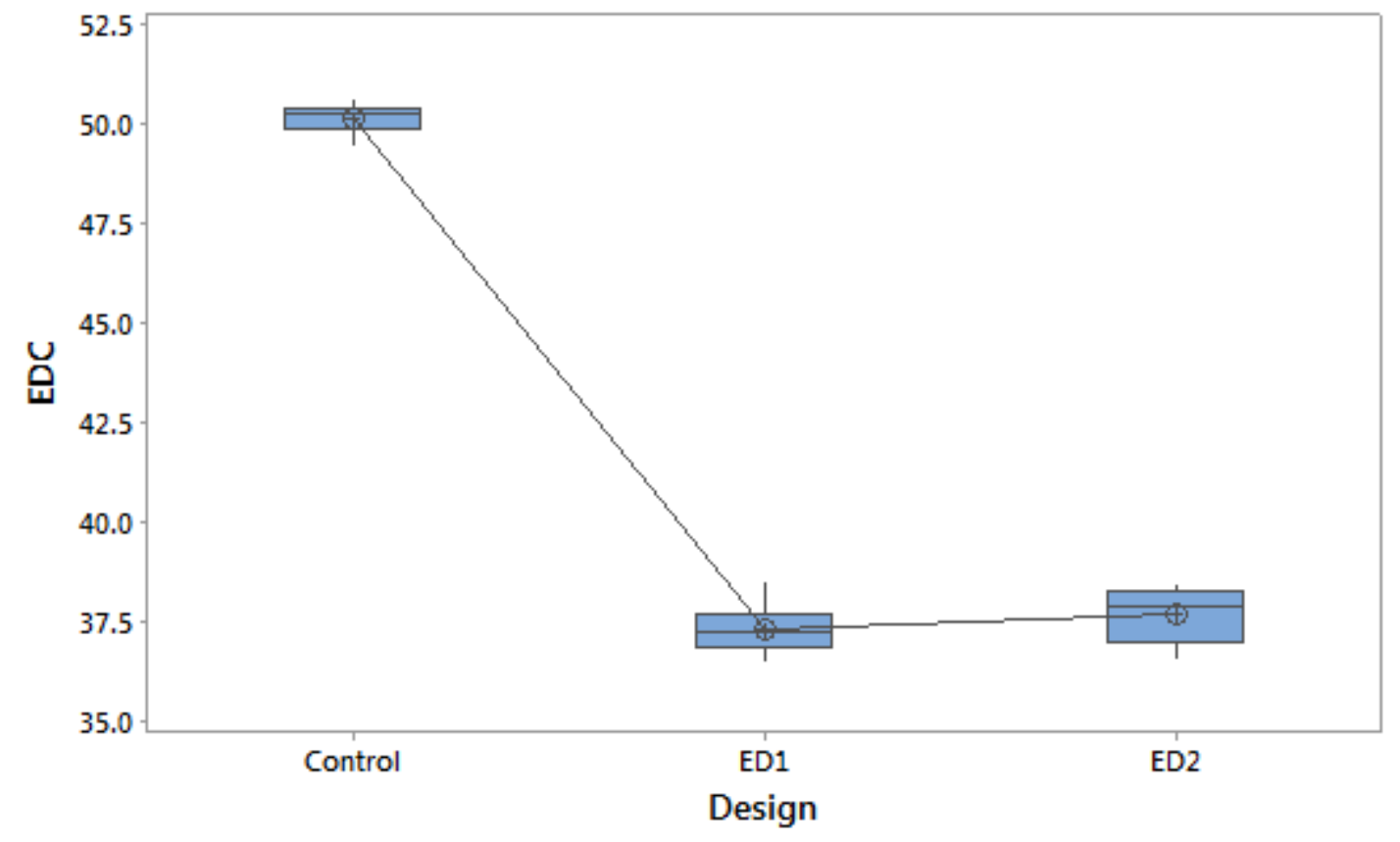

Figure 11. Boxplots for SI and EDC for 2ft drop heights 


\subsection{Test Specific Conclusions}

The small scale tests were conducted to get an idea of whether the design enhancements present in ED1 or ED2 reduce the SI values of the potential designs. In order to make a conclusion from the data that were collected during the small scale tests, a one way ANOVA was used. From the ANOVA, a Tukey comparison was run. These statistical analyses were run in Minitab 17. The Tukey comparisons are able to compare the control, ED1, and ED2 all to each other and determine whether the means of each sample are statistically different from the other means. 


\section{Tukey Simultaneous 95\% CIs}

Differences of Means for SI

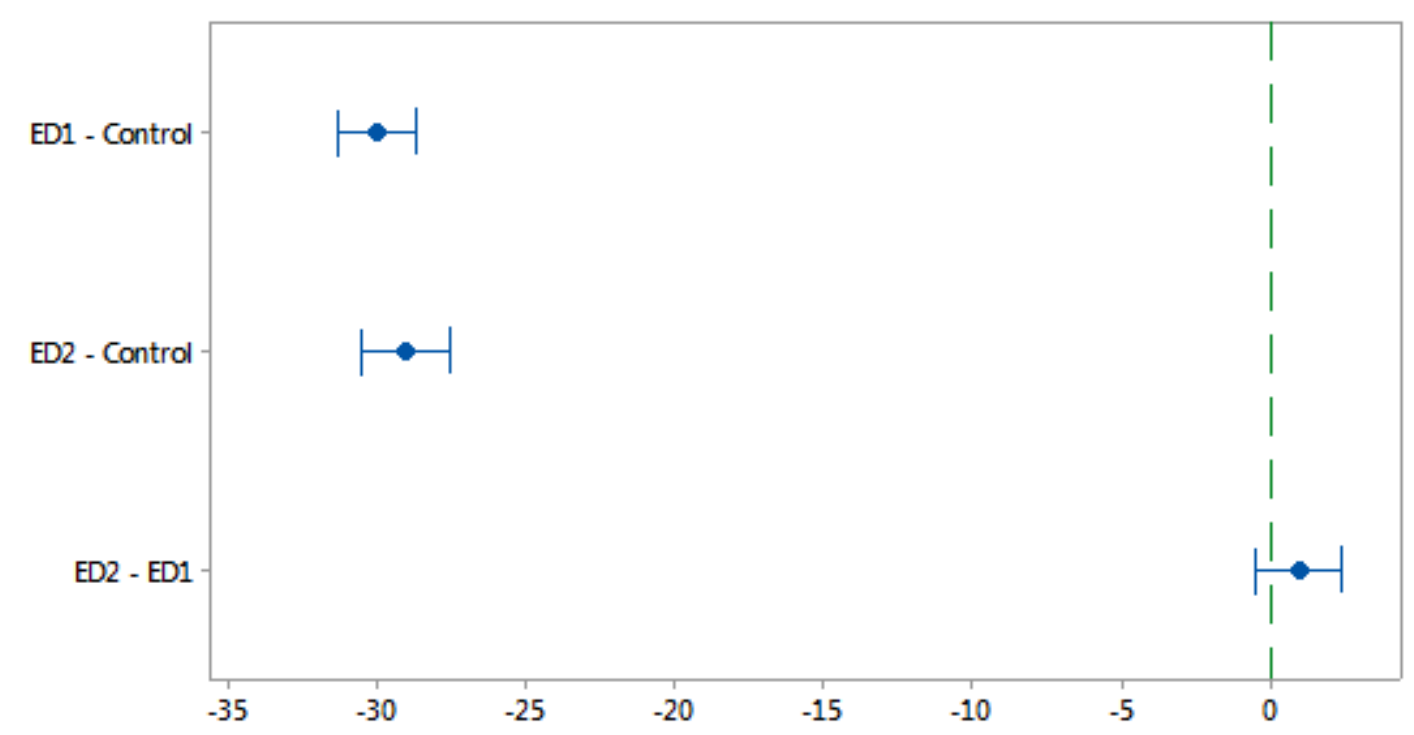

If an interval does not contain zero, the corresponding means are significantly different.

Tukey Simultaneous 95\% CIs

Differences of Means for EDC

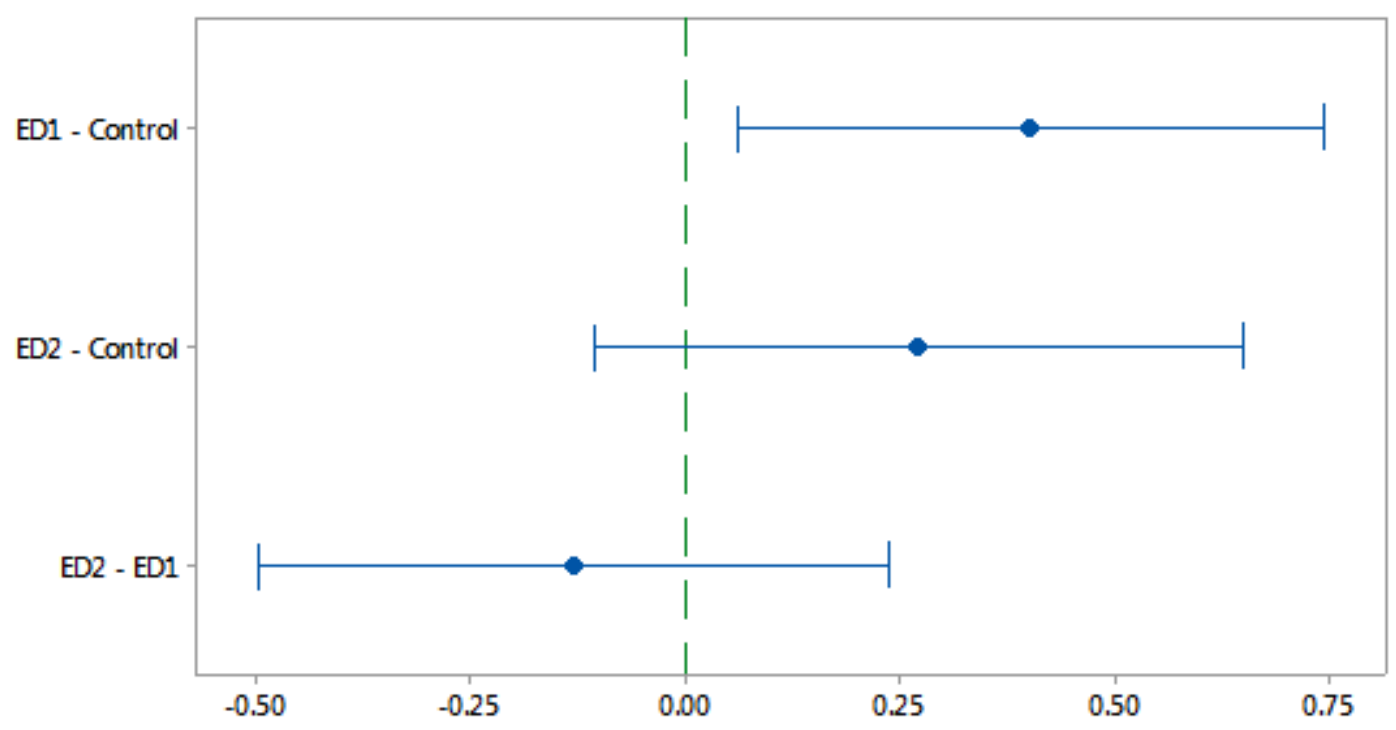

If an interval does not contain zero, the corresponding means are significantly different.

Figure 12. Tukey comparison plots for 1ft drop height of SI and EDC 


\section{Tukey Simultaneous 95\% CIs}

Differences of Means for EDC

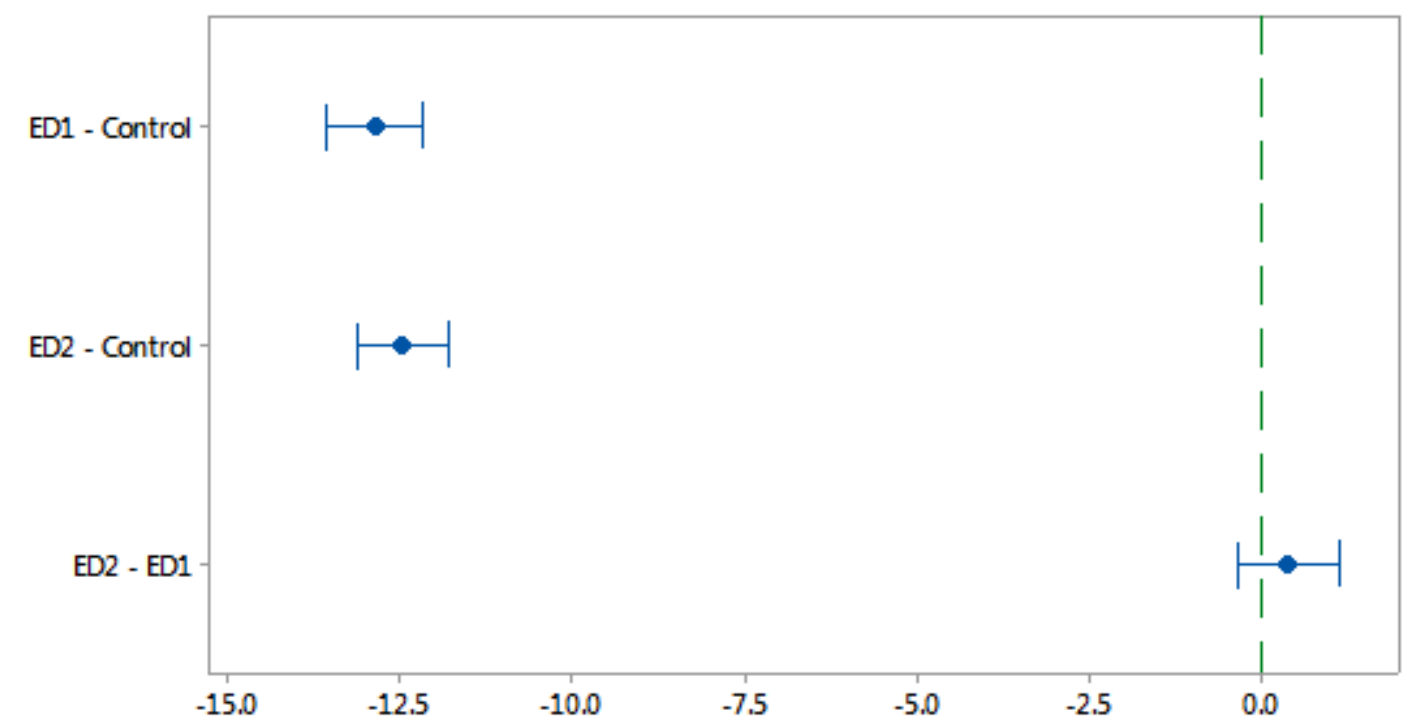

If an interval does not contain zero, the corresponding means are significantly different.

\section{Tukey Simultaneous 95\% CIs}

Differences of Means for SI

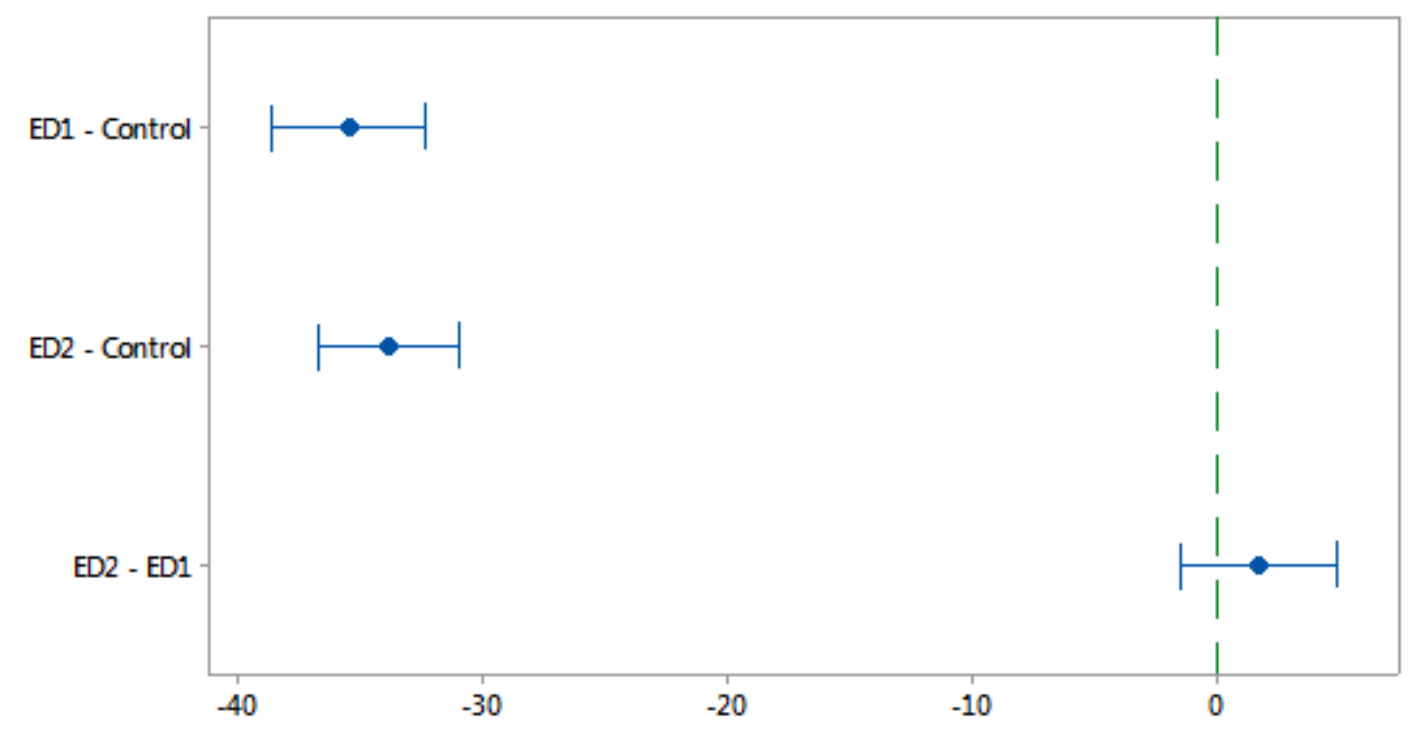

If an interval does not contain zero, the corresponding means are significantly different.

Figure 13. Tukey comparison plots for $2 \mathrm{ft}$ drop height for SI and EDC 
Figure 12 shows the conclusions from $1 \mathrm{ft}$ drop tests. It shows that for SI, both ED1 and ED2 perform better than the control helmet. The SI is reduced by $18 \%$ by using the design of ED1 versus the control. ED1 and ED2 are not statistically different. It also shows that with respect to EDC, the ED1 specimen performs better than the control but ED2 does not.

The conclusions from the 2ft drop tests are shown in Figure 13. The two foot drops show a statistical difference between the control and the ED1 and the control and ED2, but not between ED1 and ED2. The reductions in SI values versus the control are 8\%. There is also 1\% increase in EDC between the experimental designs and the control.

Between the $1 \mathrm{ft}$ and $2 \mathrm{ft}$ drop test regimes conclusions can be made about the specific test presented in the small scale testing. It was determined that both ED1 and ED2 reduce SI value. However, the differences between ED1 and ED2 are negligible. Because of this, ED2 was dropped from future test regimes as well as the computer modeling conducted by Steven Warnert. [10]

The reduction in SI was lower for the $2 \mathrm{ft}$ drop than the $1 \mathrm{ft}$ drop. As much as it may be desired to find some sort of trend in this fact, it would not be a valid conclusion. This is because an infinite number of lines can be drawn between the two points of varying curvatures. All that is known is that from a $2 \mathrm{ft}$ drop the prototypes performed worse than they performed from a $1 \mathrm{ft}$ drop. 
All the conclusions made from the small scale tests are only truly applicable to the prototypes used in the testing. The prototypes that were provided by the project sponsor were very different from the way the designs would actually be implemented in real production. This means that the conclusions made from the small scale tests must be taken with the knowledge of their limitations, such as the non-real world proportions of the prototypes and the incorrect material. 


\section{FULL SCALE TESTING}

It was determined that full scale testing was required in order to make a final determination on whether Mr. Bartholomay’s design enhancements have merit. Unfortunately, Cal Poly did not possess a device which would be able to accommodate the testing of a full size helmet. It was decided a purpose built impact tester would be constructed to accomplish the required testing. The design and manufacture of this apparatus was a major portion of the work for this thesis.

\subsection{Background}

Full scale testing of football helmets is the standard test method used in industry. These tests are done to either NOCSAE or ASTM standards with NOCSAE being the most common. Testing of football helmets has become a top priority for the sports industry to achieve reductions in concussion risk.

\subsubsection{Regulatory Standards}

The full scale testing of the designs was done as close to the standards set forth by NOCSAE in Document 001. The drop test device specified by NOCSAE is designed by the Southern Impact Research Center, or SIRC, and is a twin guide wire drop tower. The drawings provided by SIRC can be found in APPENDIX C. The drop tower specified by ASTM is very similar to that specified by NOCSAE, seen in Figure 14. [11] 
The requirements that the helmets must be tested to are specified in NOCSAE Document 002. [8]

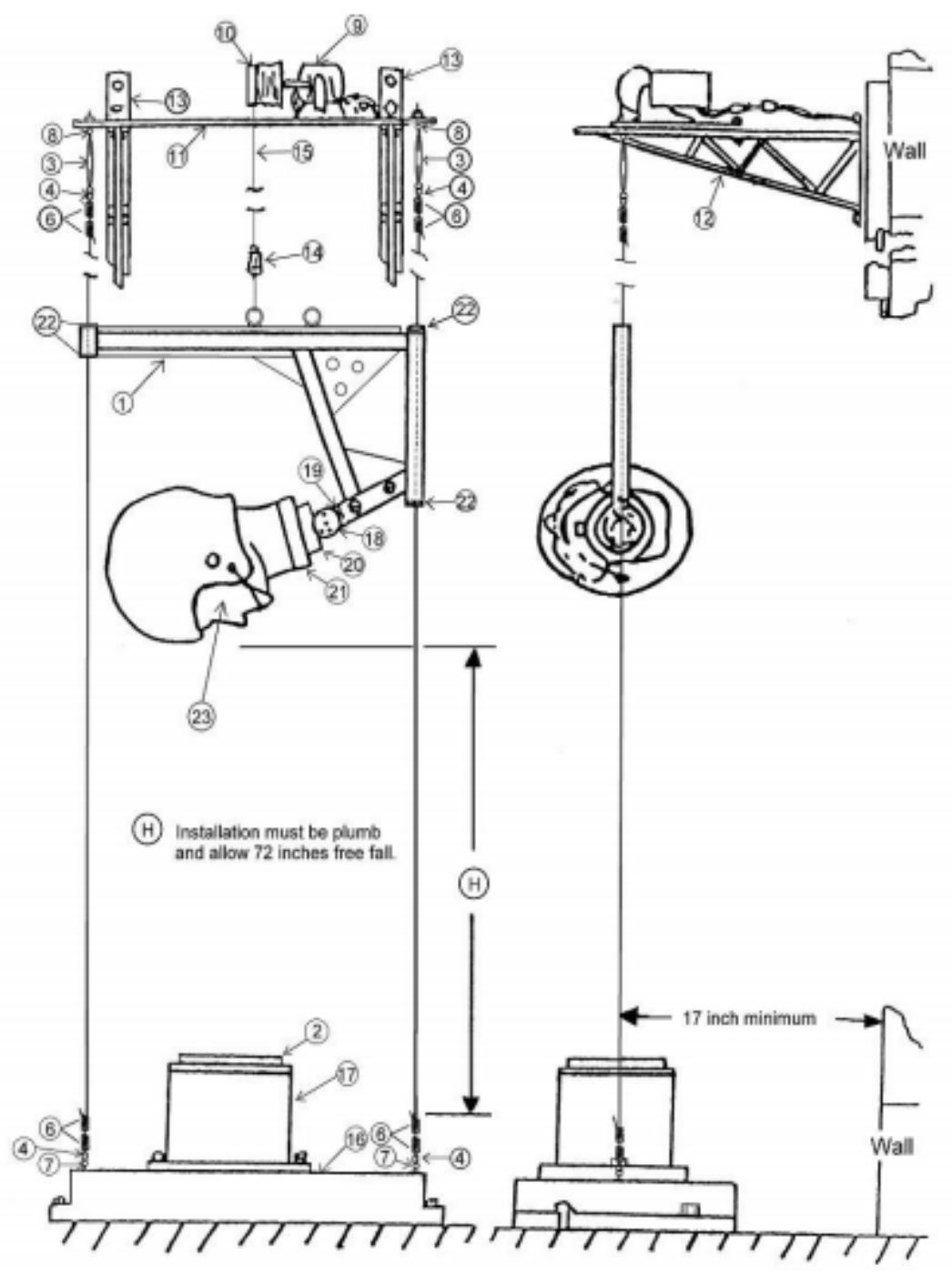

Figure 14. NOCSAE specified drop tower design

\subsubsection{Other Helmet Testing Methods}

There are two main categories of helmet test machines: drop towers and impactors. A drop tower uses gravity or other methods to accelerate the helmet into an impact surface 
guided by some sort of system into the surface. Impactors instead move an impactor into the helmet while the helmet remains mostly stationary.

There are two main types of drop towers, the twin wire and the monorail. Both NOCSAE and ASTM football helmet testing specifications specify twin wire systems. [3] [11] Twin wire systems use two tensioned wires as the guides to ensure the helmet impacts the correct spot. Wire guided towers allow the helmet to move slightly more freely due the flexible nature of the wires. Monorail towers on the other hand use a single rigid support with a roller system to guide the helmet into the impact surface. This system is much more rigid.

Impactors come in several types. Everything from pneumatic rams to pendulums are used to accelerate an impactor into a helmet. In many of these systems the helmet is mounted on a crash test dummy headform, sometimes able to slide to simulate the compliance of the human neck. Impactors specifically made for baseball batting helmet testing fire baseballs with pneumatic cannons into the helmet.

\subsection{Apparatus Design and Fabrication}

In order to carry out the testing of full scale helmets a testing apparatus had to be designed and built. In order to stay as true as possible to the design set forth by NOCSAE, the design was based upon NOCSAE's design with some modifications for 


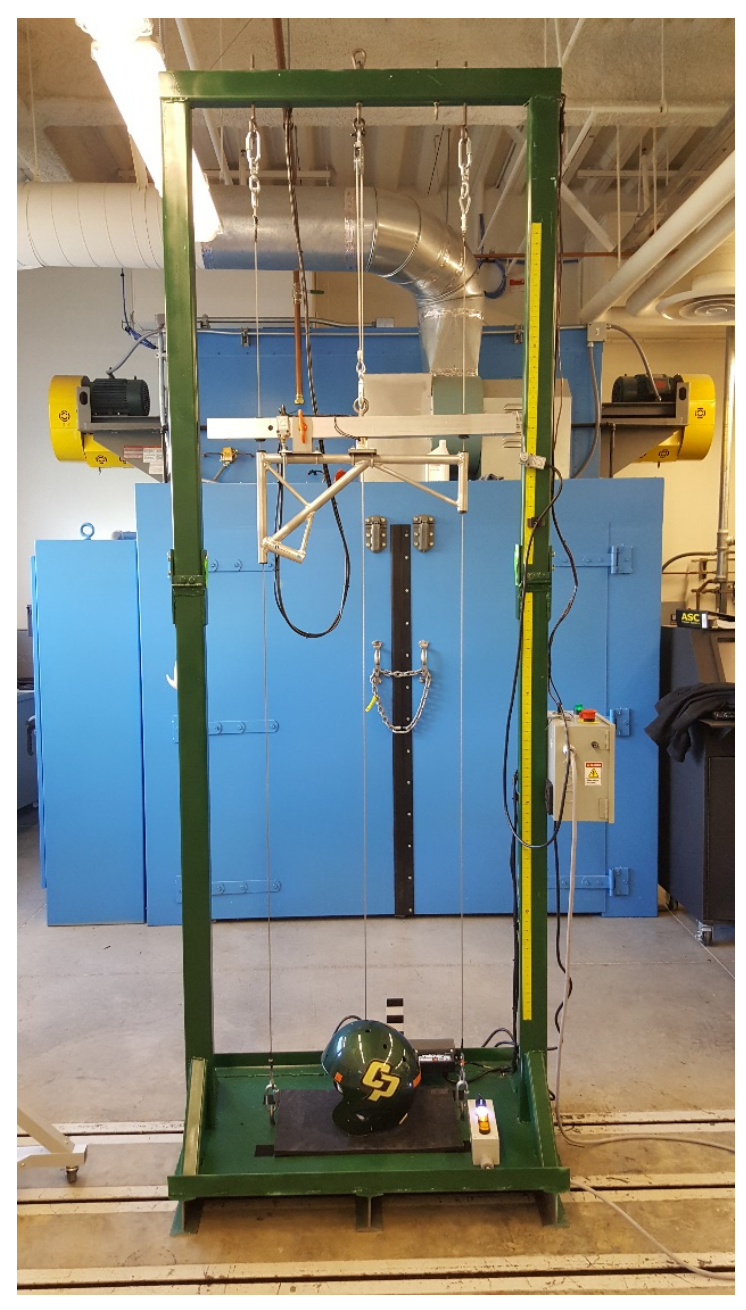

Figure 15. The NOCSAE style drop tower designed for Cal Poly

Cal Poly’s purposes. Specifically, changes were made for portability and cost. Normal NOCSAE drop towers are fixed in place and permanently attached to a wall; this new design, however, can be moved anywhere and only requires four floor mounting points. The drawings for the design can be found in APPENDIX A and the tester can be seen in Figure 15. The drop tower was funded by grants from a College of Engineering R-IDC, Returned Indirect Cost, grant and a Cal Poly university Baker-Koob grant. A majority of the apparatus was constructed by Michael Schuster with assistance from Steven Warnert 
and the Mechanical Engineering shop technicians in the Mechanical Engineering department's machine shops. The Bill of Materials is included in APPENDIX D: Helmet Drop Tower Engineering Drawin.

\subsubsection{Frame}

The frame of the drop tower is one of the main differences between the drop tower designed for Cal Poly and the standard drop tower per NOCSAE specifications. In the NOCSAE specifications the drop system is attached to a wall and the floor. This was not possible at Cal Poly due to university rules not allowing things to be attached to the buildings. Attaching the test fixture to a building also would require a space where the fixture could stay permanently.

To accommodate a portable system a frame was designed. Its purpose is to hold the entire system together. The frame supports the system as well as provides a method of rigidly attaching the system to the ground. There are three parts of the frame: the baseplate and lower frame, the hinge assembly, and the upper frame. 


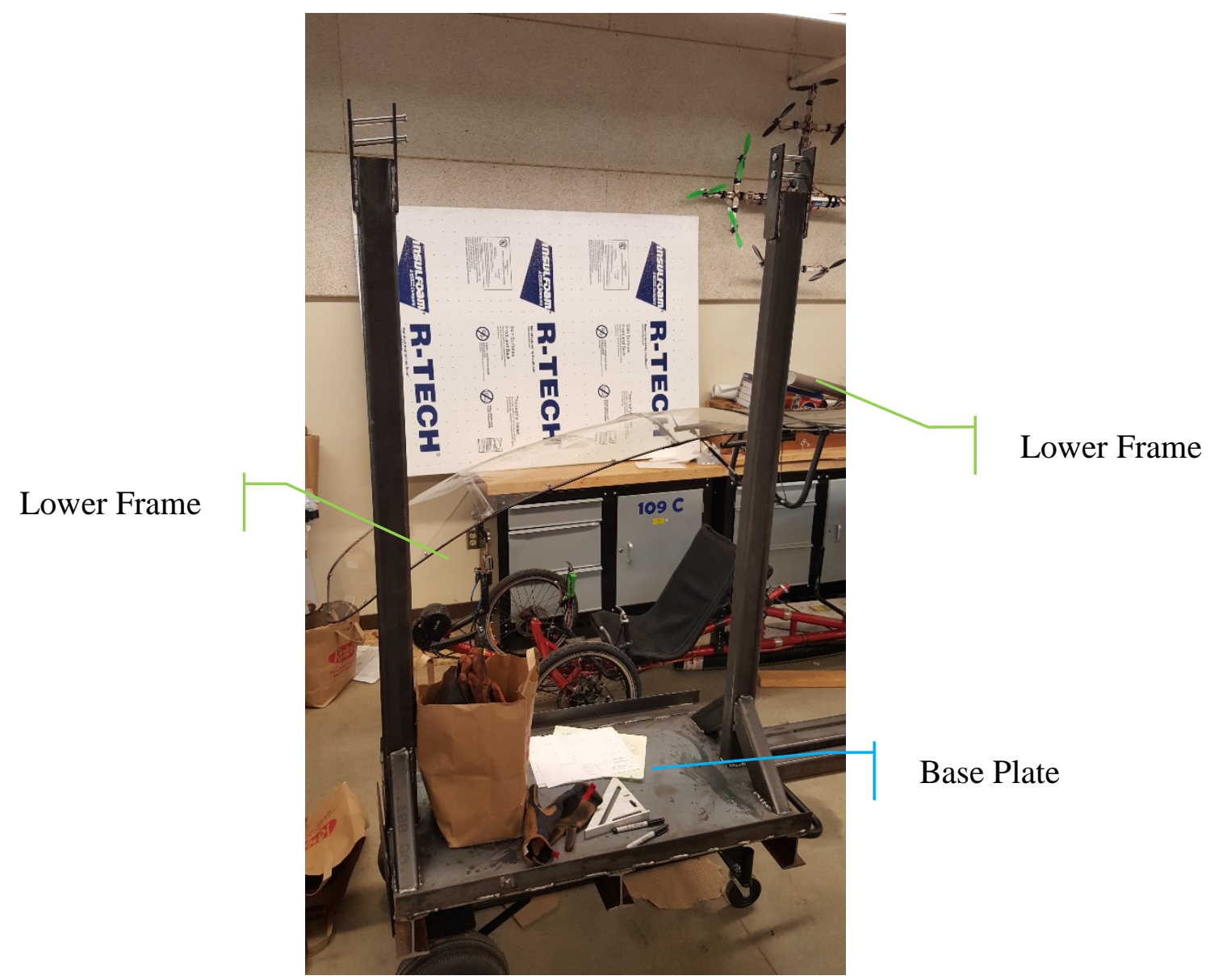

Figure 16. Bottom frame of the drop tower

The baseplate and lower frame is the bottom portion of the structure and can be seen in Figure 16. Starting from the bottom is the base. The base is sized similar to a standard pallet. This allows a standard forklift or pallet jack to move the system around campus. Resting on top of the base is the impact surface, a Buna-N rubber pad. This pad is specified by NOCSAE to be a durometer of $38 \mathrm{~A} \pm 5$. The actual pad that NOCSAE specifies is called a MEP pad. [3] This pad was not used because it was too expensive, costing around \$600 for a single pad. Another difference from the standard NOCSAE design is in the baseplate. Whereas the NOCSAE design uses a sliding anvil as an impact surface, this design uses the top surface of the baseplate with a much larger impact area. 
There are mounting holes on the baseplate which allow the structure to be rigidly attached to a strong floor with a one-foot rail spacing. Additionally, concrete anchors could be purposefully sunk to attach the structure to the ground. Bolting the structure to the ground allows it to be lighter without having to worry about the structure moving during impacts. Attached to the base plate are the two lower frame beams. Like the rest of the frame beams, the lower beams are made from $2 \times 3$ inch steel tubing with 3/16 inch walls. This tubing was chosen because of availability and cost.

The hinge system connects the upper and lower frame sections as seen in Figure 17. The

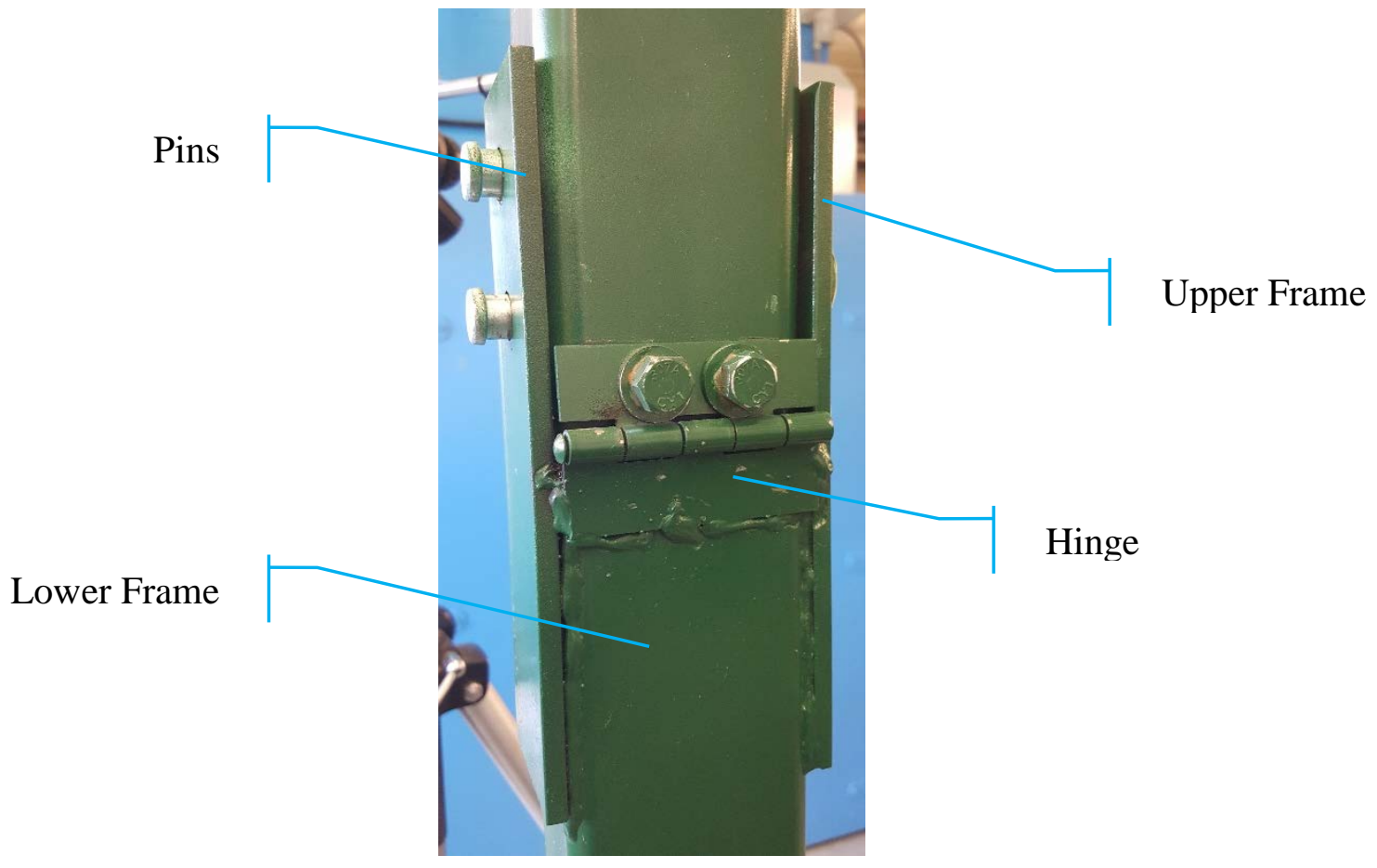

Figure 17. Hinge section of frame

purpose of the hinge is to allow the frame to collapse to a height which will fit through a standard door frame. This adds to the portability of the system. The lower portion of the hinge is welded to the lower frame and the upper portion is bolted to the upper section. This enables slight misalignment of the frame sections. The sections are held upright 
with clevis pins running through plates welded to the lower frame and cotter pins. These pins are supported in double shear and ideally support limited load if the frames are properly aligned and assembled. This is due the fact that the upper frame will rest on the lower frame when assembled.

The upper frame is made of three beams. The two side beams are bolted to the hinges. The top beam is drilled to accept the eye bolts from the drop system. The entire frame can be seen in

Figure 18.

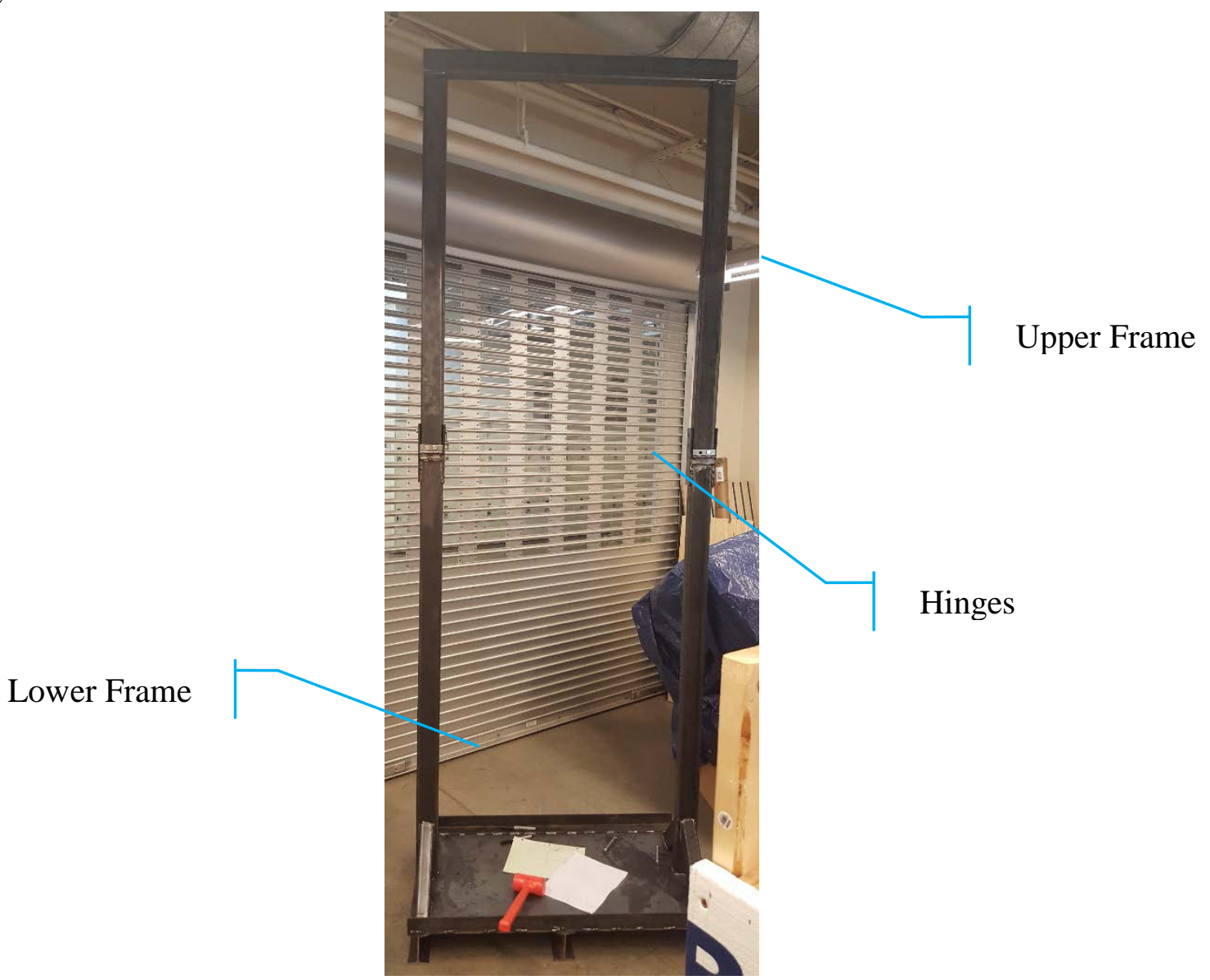

Figure 18. The entire unpainted frame 
The frame was analyzed for both bending of the top beam and buckling of the upright supports. The cables of the drop system are tensioned to 300lbf [11]. This specification is per ASTM standards as NOCSAE does not specifically give a tension requirement. These calculations gave a factor of safety for buckling of greater than 8 and a deflection in the top beam of around 0.006 inches. Both of these values are acceptable.

\subsubsection{Drop System}

The drop system consists of all of the rigging and parts used to actually drop a helmet into the impact surface. The drop system has the following major parts: the cabling, the lift bar and winch, and the carriage.

The cabling consists of the steel cables and the rigging required to keep the cables under

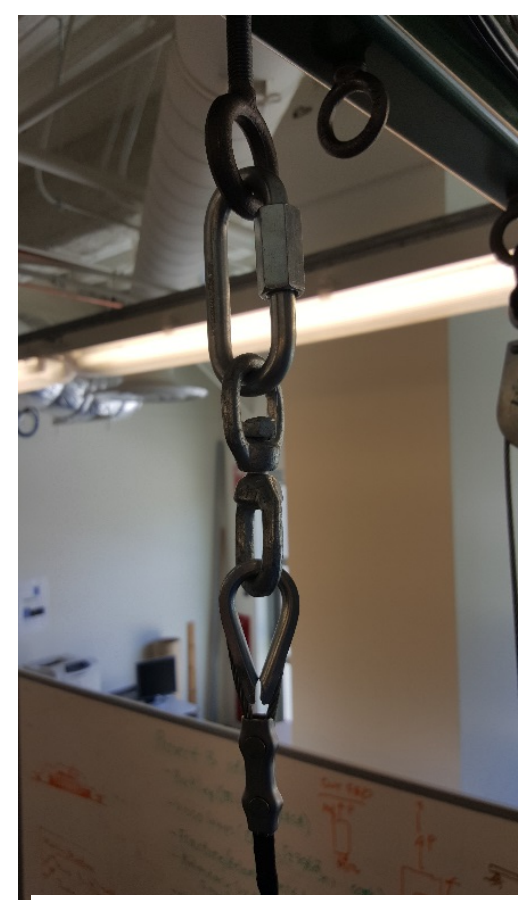

Figure 19. The cable rigging to connect the cable to the upper frame

tension. The cables themselves are one-eighth inch diameter galvanized steel cables with 
tensile strengths of 400lbf. These cables were chosen because they were the strongest commonly available cables with the required diameter. To keep the cables under tension, an eyebolt with a long thread is run through the top beam of the frame. Each eyebolt is then tightened with a serrated locknut to provide the tension. The base of the cabling is attached to an eyebolt in the baseplate. The cable ends are finished with thimbles to protect the cables and wire clamps. To attach the cables to the eyebolts screw links are used. Between the cable and the top screw link is a rotating link to allow the top eyebolt, which provided the tension, to be turned. The upper rigging is shown in Figure 19. The cabling tension was not measured for this series of testing due to the lack of a method to measure it. There are commercially available devices designed for measuring the tension in rigging, such as sailboat rigging, which could be used but were unavailable for this

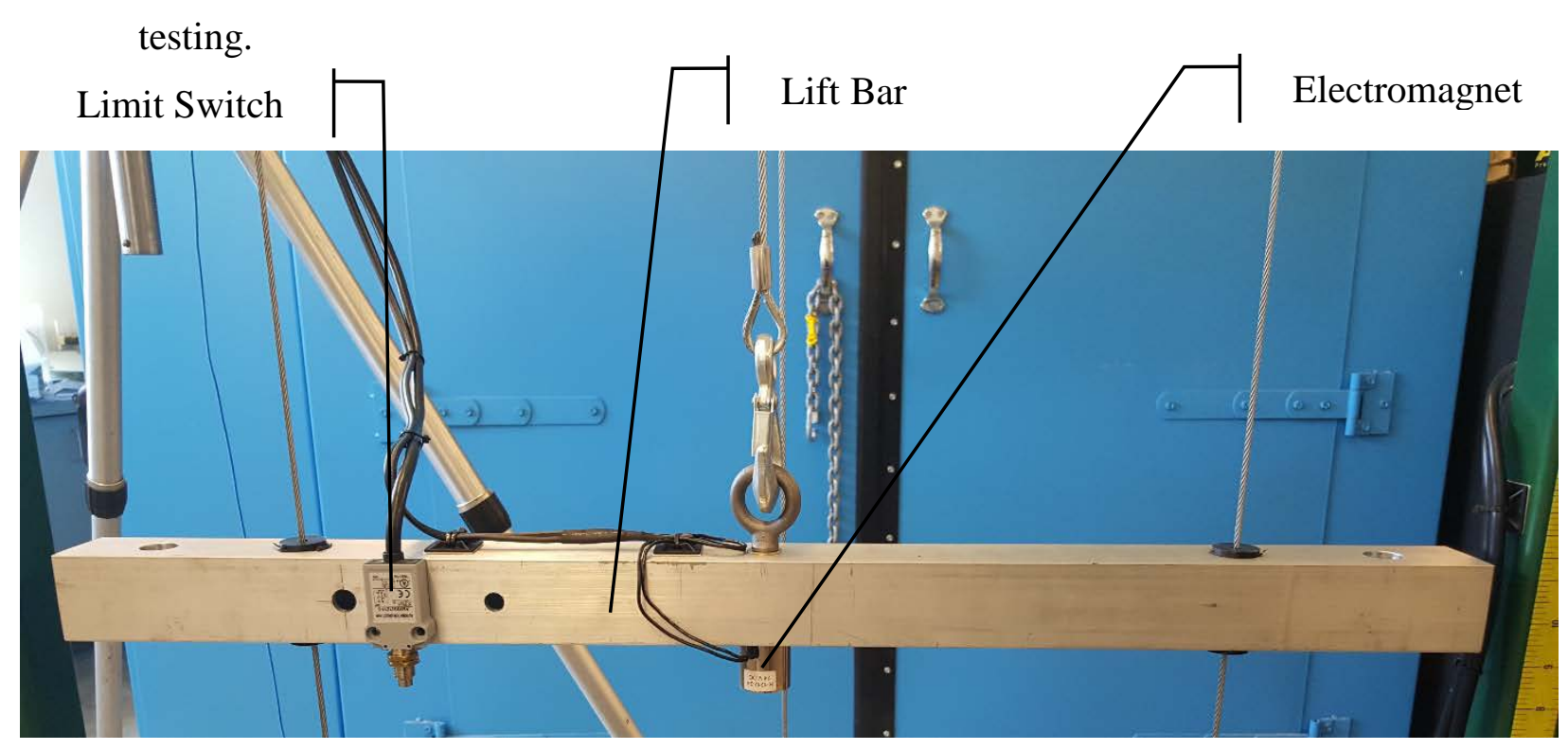

Figure 20. The lift bar showing the electromagnet, the limit switch, and the eyebolt

The lift bar is the assembly which is responsible for lifting and releasing the carriage, it is seen in Figure 20. It is an aluminum bar which runs along the guide wires. To reduce the 
friction with the cables, delrin bushings are used. These bushings were designed by SIRC. The bar is lifted and lowered by the winch, which is attached by an eyebolt screwed into a threaded insert. The carriage is held in place as well as released by an electromagnet. The magnet has a pull force of 50lb. The magnet attracts a steel plate attached to the carriage. Additionally, the lift bar holds a limit switch to stop the winch from being able to lower it once the carriage is engaged. The lift bar is under very little stress since the only load is the aluminum carriage and so was not analyzed for strength.

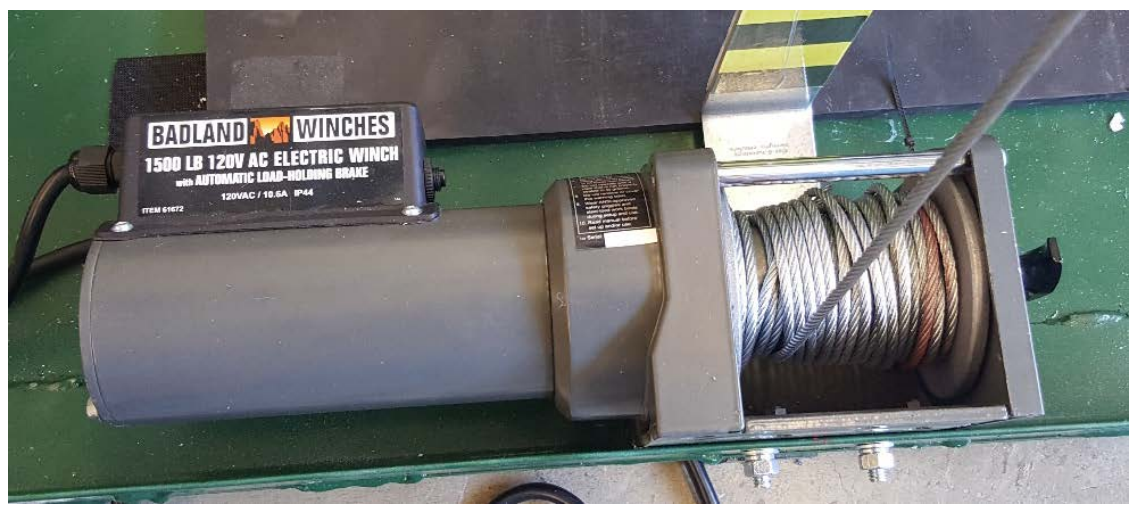

Figure 21. Harbor Freight winch responsible for positioning of lift bar

The winch, shown in Figure 21, is a standard 120 VAC winch available from Harbor Freight. It is rated for 1500lb but is only used to lift the lift bar and carriage which together weigh less than $2 \%$ of the rated load. The only modification made to the winch was the removal of the standard control pendant and wiring it into the control system. The winch is mounted to the angle iron on the base plate. The winch cable is run through pulley attached to the top bar of the frame with a shackle. 


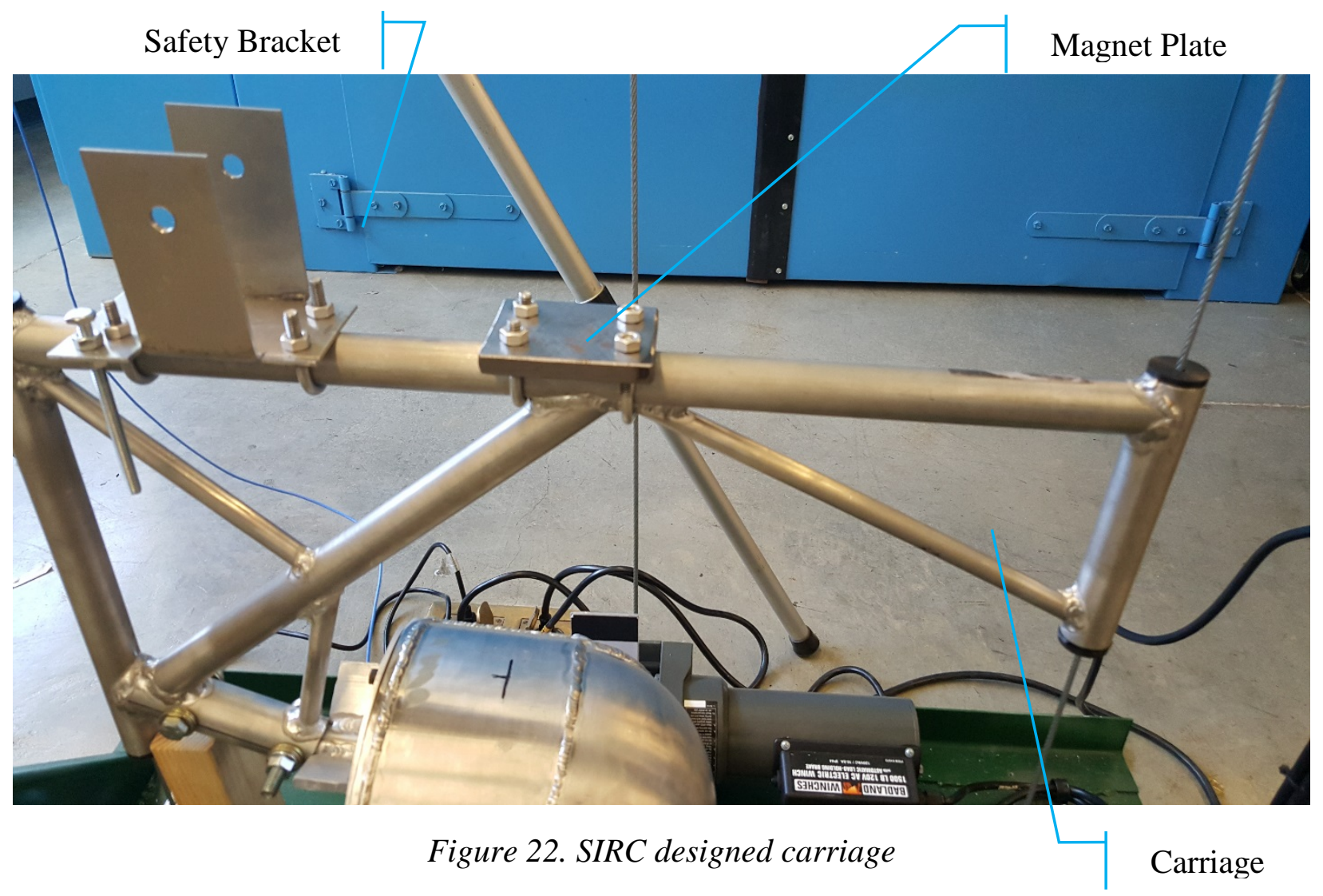

The carriage is the part of the drop tower which actually drops. The carriage holds the headform or any other item being tested. The carriage with the headform attached is shown in Figure 22. The carriage for the most part was designed by SIRC and is made mostly of Aluminum 6061. The SIRC design was chosen because it matches the requirements set forth in the NOCSAE standards and would be the hardest part to change if full certification was desired on the machine. Two parts were added to the carriage. The first is a steel plate attached to the top bar near the middle of the carriage. This plate is what allows the electromagnet to attract the mostly nonferrous carriage. Also attached is the safety bracket. This bracket serves two purposes. The first purpose is the enable a pin to be run though it and the lift bar to ensure the carriage cannot drop even if the electromagnet loses power. This increases the safety for an operator working on the machine. The bracket also is what pushes the limit switch on the lift bar. A screw can be 
adjusted up and down so that the limit switch is activated in the correct spot. Both the added features, the magnet plate and the safety bracket, are attached to the carriage with aluminum u-bolts. The carriage was designed by SIRC and is used on many drop towers so did not need any further analysis of its strength.

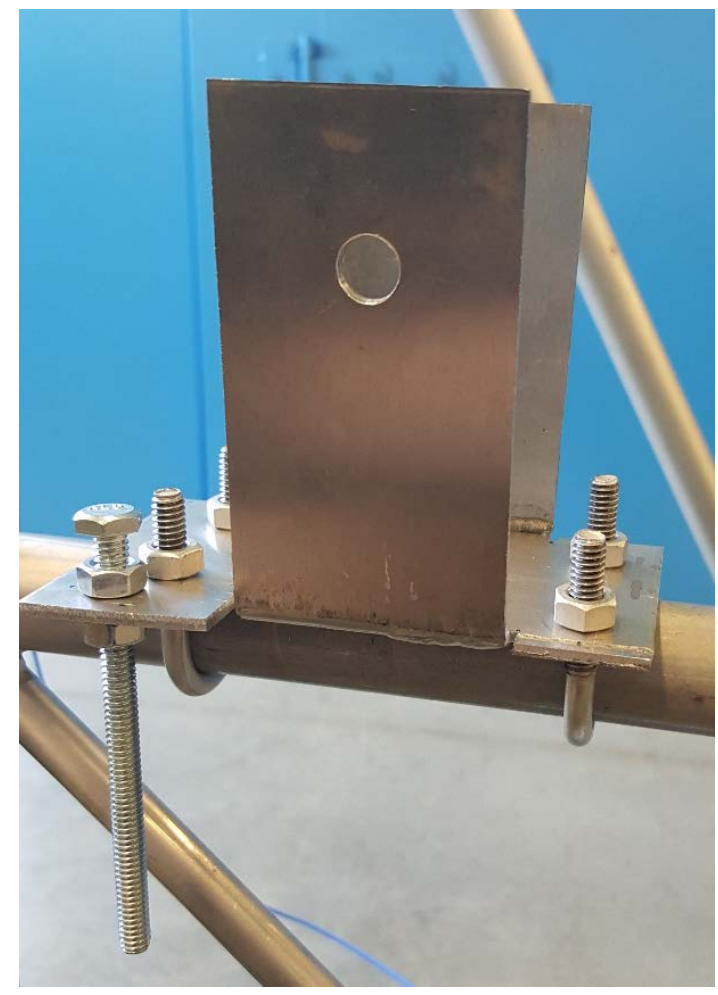

Figure 23. The safety bracket attached to the carriage 


\subsubsection{Headform}

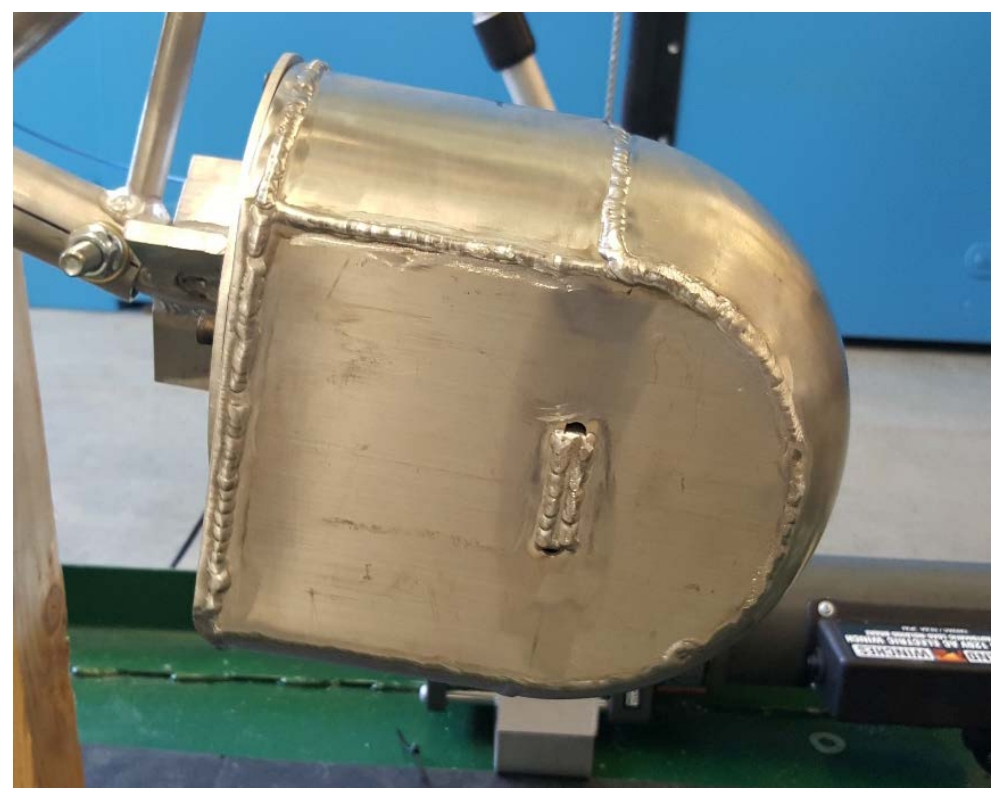

Figure 24. Cal Poly drop tower's headform

The headform is the structure which wears the football helmet. It is the approximate dimensions of a human head. The actual headform that is called out in the NOCSAE standards and used in the SIRC designed drop tower proved to be overly expensive so a work around was designed. The headform that was designed for the Cal Poly drop tower is made out of aluminum. It is specifically made out of a hemisphere, made by metal spinning, pieces of large diameter tubing, and plate. Inside of the headform is a shelf which holds the accelerometer. To align the accelerometer with the head, the shelf was polished so that screw stud could be aligned properly. The headform is designed to be attached with a movable neck joint to allow different surfaces of the helmet to be impacted. The neck is designed by SIRC, called the stem and rotator. These parts were required to be CNC machined and were not completed in time for testing due to manufacturing errors. To act as a stop gap measure, a simple non-articulating stem was 
welded in place. The parts were not completed even at the completion of the testing for this project.

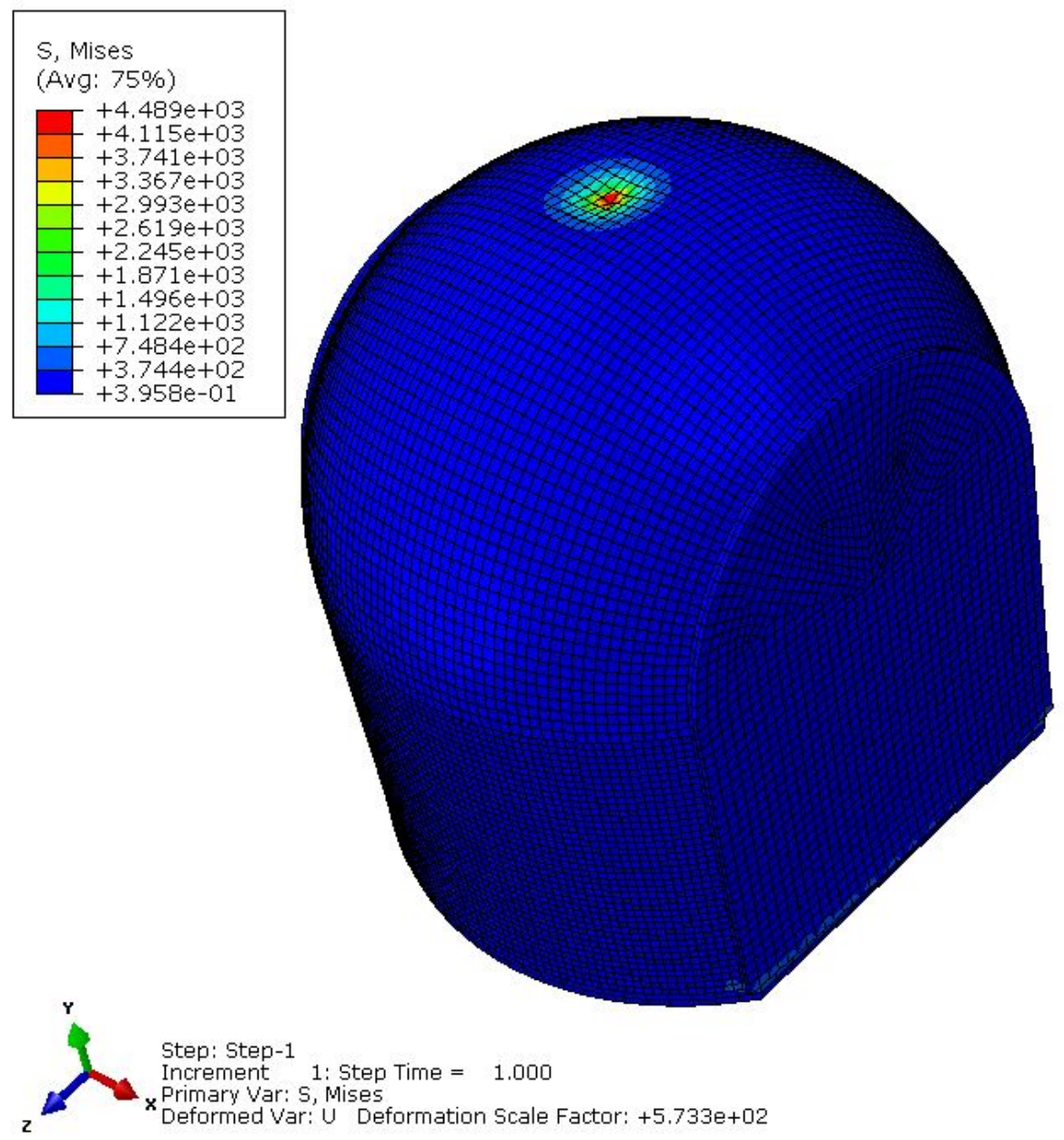

Figure 25. FEM of headform

Since the headform endures the brunt of the impact forces it was analyzed for strength.

The analysis of the headform proved very difficult by hand. Because of this it was decided to use a simple finite element model to study the yield of the headform. The model was built using 3D brick continuum elements with all the pieces of the headform tied together, to simulate the welds. A 50lbf was applied to the center of the hemisphere. This load was applied because it is the load equal to a drop from $17 \mathrm{ft} / \mathrm{s}$ with an impact 
time of 17 milliseconds. The model can be seen in Figure 25. The headform carries a factor of safety of 4 for yield based upon the yield strength of Aluminum 3003. In actuality, the force imparted upon the headform would be less due to the padding in the helmet.

Using the helmet in actual testing has proved that the headform itself has undergone no damage. The only damage is to the mounting stem, which will be discussed later.

\subsubsection{Control System}
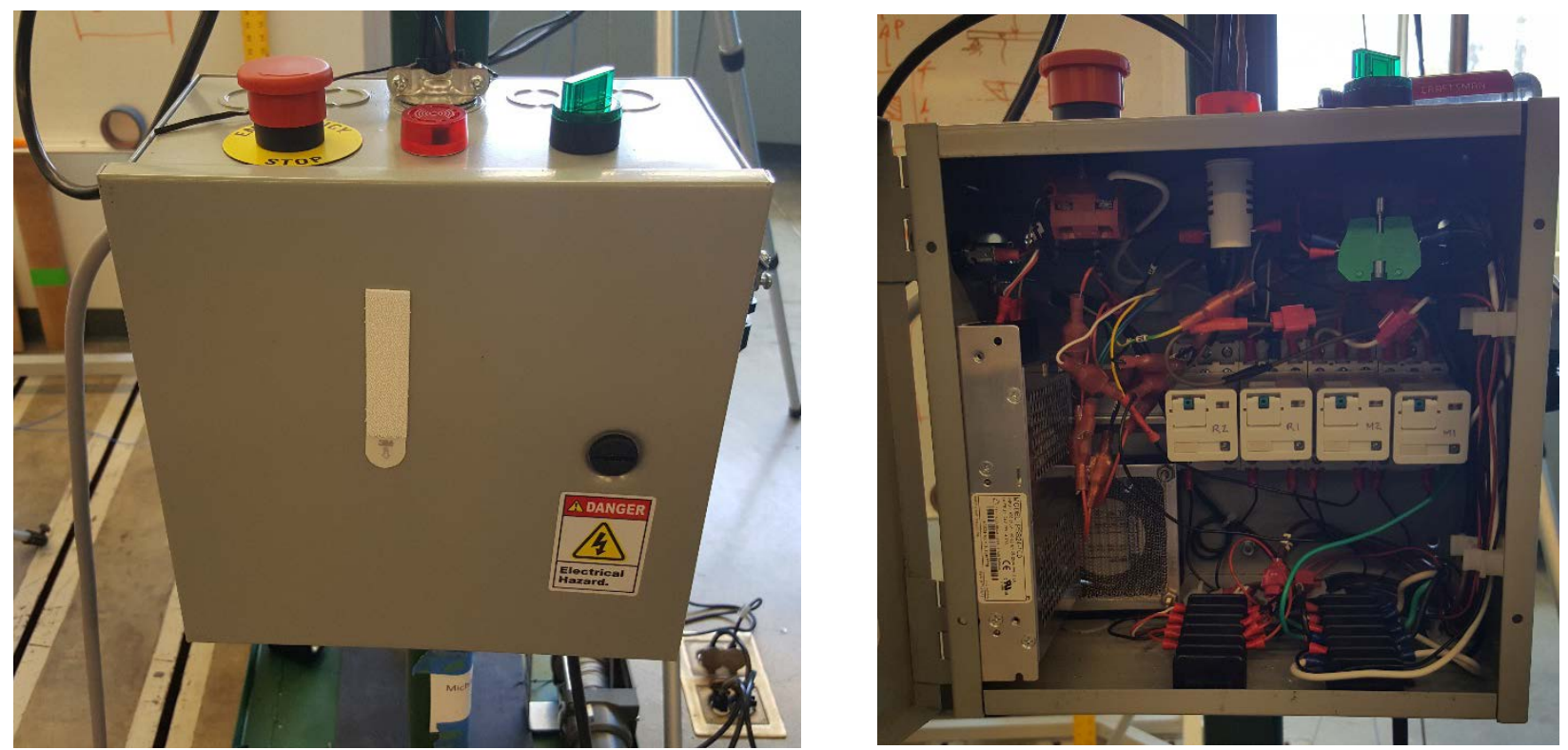

Figure 26. The outside and inside of the control system enclosure

The control system has two functions, each with its own circuit. Both circuits are contained inside an enclosure, show in Figure 26, mounted to the lower frame of the drop tower. The entire machine operates off of a single 120 volt power cord. To cool the enclosure, a 120 volt fan is on at all times while the machine is running. A single power switch activates all circuits in the control system. The system has analog control, using 
three electromagnetic relays. The control system was checked for safety by Ben Johnson, Cal Poly’s supervising electrician.

The first function/circuit is for winch motor control. This system replaces the traditional pendant on the winch to allow all the controls for the system to be encased in one pendant. To replace the pushbuttons of the original pendant, two relays were installed. One relay sends power into the motor one direction to winch the lift bar up and the second relays sends power in the reverse direction to lower the lift bar. The two relays are controlled by a monetary three position, center off rotary switch. When the switch is turned either direction a $24 \mathrm{v}$ signal is sent to activate the relay. Also part of the winch circuit are two limit switches. One limit switch is to stop the downward motion of the lift bar. This is a pushbutton limit switch activated by a screw on the safety bracket of the carriage. The second limit switch stops the upward motion of the lift bar. This limit switch has a metal bar which sticks out and is rotated by the lift bar. This limit switch is attached to the frame with magnets to enable its height to be easily adjusted. Both of these limits switches cut off the signal going to the respective relay shown in Figure 26. For safety, the system's emergency stop button will shut off all power going to the motor disabling it from moving either direction. 


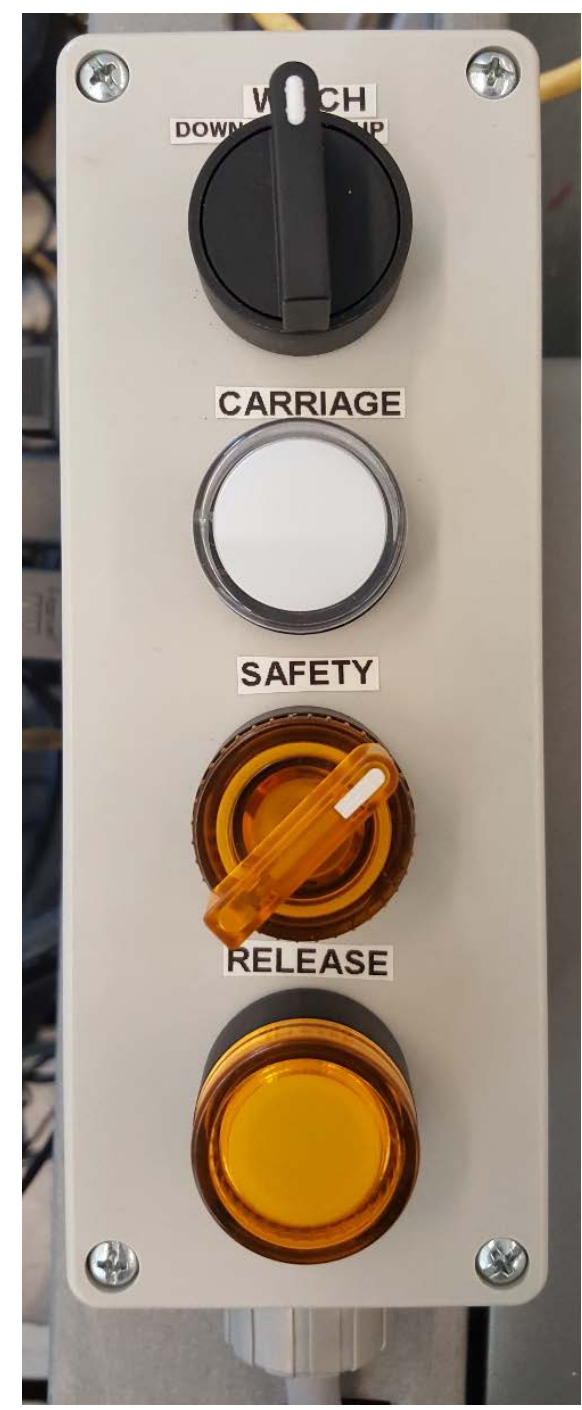

Figure 27. The drop tower control pendant

The second function/circuit is the electromagnet control circuit. In order for the electromagnet to be able to release the carriage, the polarity of the magnet must be reversed. This is because a latent charge builds up in the magnet and is still charged if the power is only shut off. If the power to the magnet were only shut off, instead of the polarity switched, there would be a lag before the carriage was released. The polarity is reversed with a relay. When power is applied to the system the relay applies power to the magnet and it builds a charge in one direction. Once the relay is activated it reverses the 
current flowing to the magnet to reverse the polarity. The electromagnet operates on 24 volt D.C. power. To create the 24VDC supply voltage there is a power supply in the electrical enclosure powered through a fuse with the 120VAC input power. There are several safety systems in place to help prevent inadvertent release of the carriage. These safety features were based on those of the Dynatup drop tester. The first safety feature is a double action release. To activate the relay both a momentary, rotary switch and then a pushbutton must be activated before the 24-volt signal is sent to the relay. Additionally, when the rotary switch is activated a buzzer and safety light turn on to enable both audible and visual indication the carriage is ready to drop. A final safety feature is the machine’s emergency stop button which when activated stops the relay from switching and the carriage from releasing. This allows the carriage to still stay attached to the lift bar without the possibility of its dropping accidently.

The control pendant for the drop tower also has light indicators. When the lowering limit switch is activating a white light, the "Carriage" light, illuminates. This alerts the operator that the carriage is now attached to the lift bar and is ready to be raised. Once the raising limit switch is activated the light on the rotary safety switch will illuminate. When both the white and orange lights are lit the operator now knows the carriage is at the right height. Once the safety switch is turned, the release button is illuminated orange in addition to the safety buzzer and light. 


\subsubsection{Instrumentation}

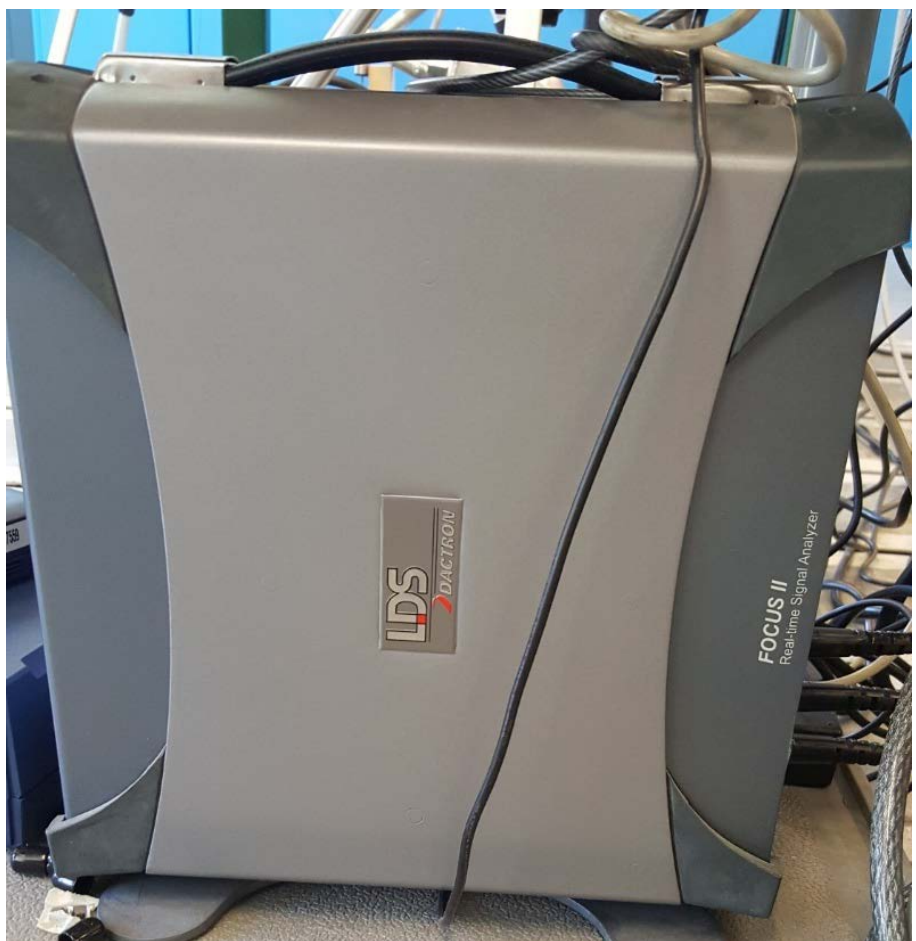

Figure 28. LDS Dactron Focus II DAQ

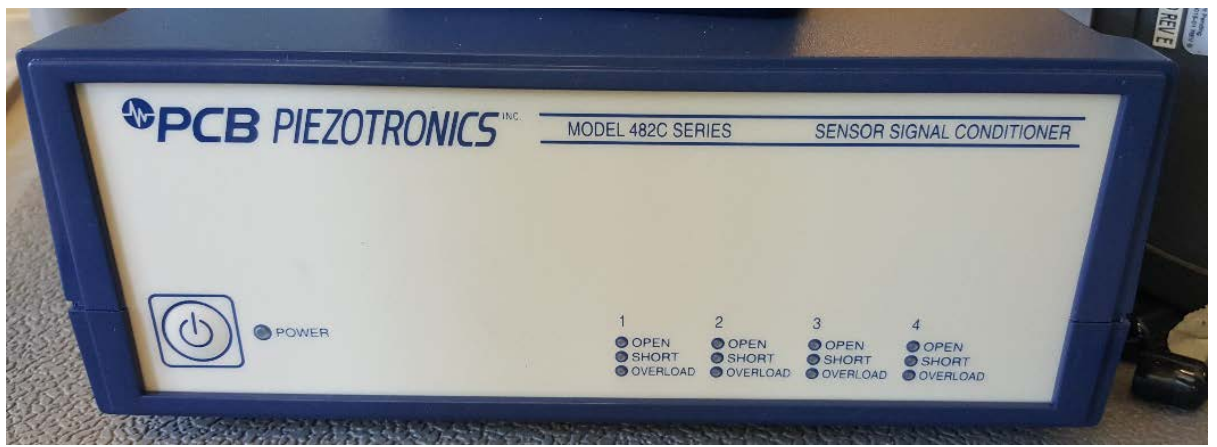

Figure 29. The PCB 482C05 Signal Conditioner

The system's instrumentation is very similar to that used for the small scale testing. The major difference is that the full scale drop tower uses a three axis accelerometer versus the small scale testing's single axis accelerometer. The change to a three axis accelerometer allows the instrumentation to match that required by the NOCSAE standards. [3] Using a three axis accelerometer also allows for varying orientations of the 
headform so that the helmet can be impacted in multiple locations. The accelerometer used is a PCB 356A02. The specification sheet for this accelerometer can be found in APPENDIX F: Specification Sheets. The accelerometer signals are sent through a PCB 482C05 ICP signal conditioner before being recorded by the LDS Dactron data acquisition system, shown in Figure 29 and Figure 28 respectively. The sampling frequency for the full scale testing was 16384 hertz, just like the small scale testing, due to the SAE impact instrumentation specification. [9]

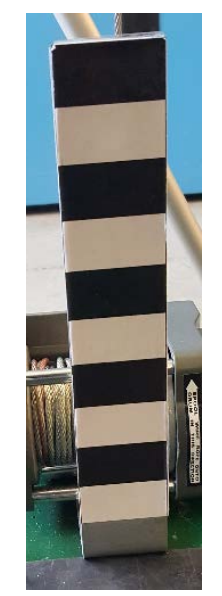

In addition to the accelerometer system required by the NOCSAE standards, a high speed camera was used. [3] The high speed camera was used to determine the actual drop velocities of the helmet as well as to visualize the movement of the helmet. To determine the speed a photoscale was used. The scale is a series of one inch bars attached via magnets to the baseplate, it was positioned directly behind the helmet.

Figure 30. Photoscale for velocity measurement

\subsubsection{Verification}

In order to ensure the drop tower was consistent between tests, a series of verification tests were run. These tests were run using a surplus Cal Poly football team helmet. The helmet was dropped four times from a two foot height and five times from a four foot height. The SI values from these tests are in Table 3. 
Table 3. Drop test machine verification runs

\begin{tabular}{|c|c|c|}
\hline Run & 2ft Drop SI (159 in/s) & 4ft Drop SI \\
\hline 1 & 555.28 & 1422.2 \\
\hline 2 & 681.34 & 1324.0 \\
\hline 3 & 642.34 & 1107.3 \\
\hline 4 & 662.80 & 1360.1 \\
\hline
\end{tabular}

For each height, the standard deviation of SI values was calculated. Dividing the standard deviation by the mean SI value gives a value which can be used as the tolerance for the drop tower. The values for the heights are presented in Table 4. The drop speed variation was negligible.

Table 4. Drop tower tolerances for both test heights

\begin{tabular}{|c|c|}
\hline Drop Height & Tolerance of SI \\
\hline $2 \mathrm{ft}$ & $14 \%$ \\
\hline $4 \mathrm{ft}$ & $10 \%$ \\
\hline
\end{tabular}

\subsection{Test Procedure}

The drop tower was designed with a specific test procedure in mind. During initial testing the test procedure designed for the machine was less practical than thought so a different procedure was used.

\subsubsection{Design Test Method}

The test method that the machine was designed for can be found in Appendix G. 


\subsubsection{Test Method Used}

The test method that was used for the full scale testing was:

1. Apply power to drop tower

2. Apply power to instrumentation
a. Signal conditioner
b. DAQ
c. High Speed Camera
d. Laptops

3. Secure Helmet to headform with duct tape

4. Raise lift bar to correct height

5. Raise carriage by hand, ensure carriage is captured by magnet

6. Measure helmet to impact pad height to ensure correct height is achieved

7. Retreat safe distance and clear area of unnecessary personnel

8. Pretrigger high speed camera

9. Start DAQ

10. Turn Safety Switch

11. Release carriage

12. When impact is heard, trigger high speed camera

13. Stop DAQ

14. Save data and video

15. Readjust helmet

16. Repeat 5-16

17. When testing completed, remove power from all instrumentation and drop tower 


\subsubsection{Test Specimens}

There were two test specimens involved in the full scale testing. These specimens were fabricated using additive manufacturing techniques. The specimens were created by a company found by the sponsor of the project. The company was chosen by the sponsor because it had a significantly lower quote to manufacture the helmets than several other more well-known companies. Unfortunately, this lower price resulted in lower quality. To carry out the testing it was desired to use prototypes manufactured with the Selective Laser Sintering, or SLS, process. The SLS process uses a laser to sinter particles of plastic together to form a complete item. The material used would have been an impact resistant nylon. The main benefit of the SLS process is the fact that it does not need to use support material while printing because the vat of plastic particles acts as its own support material.

The helmets were ultimately manufactured with the Filament Deposition Modeling, or FDM process. This process uses a plastic filament that is extruded into layers. Each layer is built on the next, ultimately building up the final part. This process uses ABS plastic as its material. This method is much cheaper than SLS and is why the sponsor chose a company which used it. There are two main problems with the FDM process, however; the requirement of support material and that the layers are not completely fused to the others. In order for an FDM model to be made support material has to be printed because the FDM printer cannot extrude plastic in midair. The layers not be fused 
together adds places which are weaker between every layer making the prototype highly anisotropic.
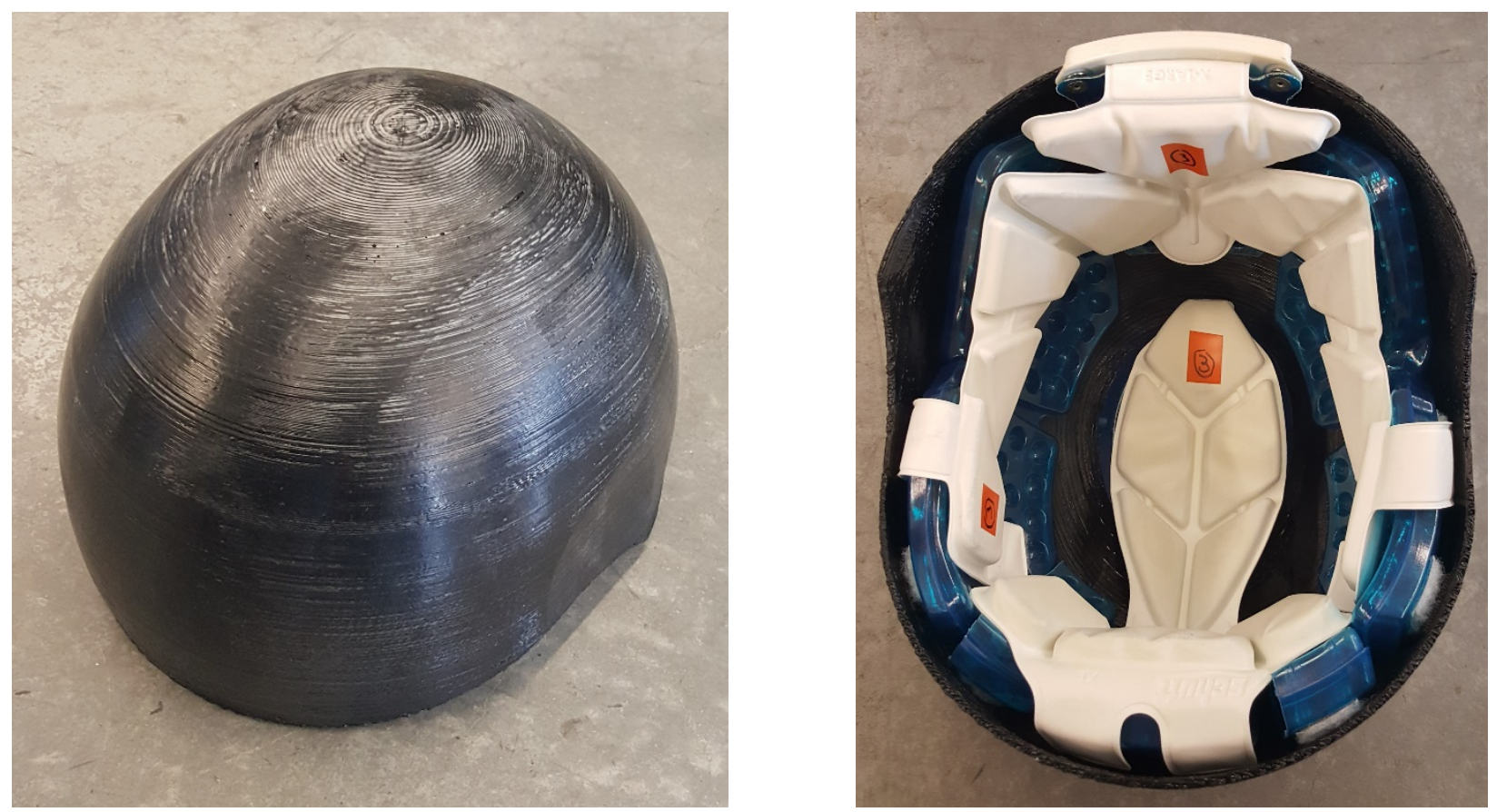

Figure 31. FDM control shell outer surface and inner surface with padding installed The additive manufactured prototypes were just shells of helmets. Since the helmet shells were made out of ABS, not polycarbonate like a real football helmet, a control helmet with no added features was made as well as the experimental. The control helmet, shown in Figure 31, was made to try to eliminate any influences due to material. To fully simulate football helmets, padding had to be added. Padding was removed from a retired Cal Poly football helmet and was attached to the inside surface of the prototype shells. The same padding set was used in both the control and experimental helmet. 
For the machine verification tests a second retired football helmet was used. The football helmet was equipped with the same type of padding used in the control and experimental prototypes. The retired helmet was a Schutt helmet made out of polycarbonate.

The prototype helmets produced unfortunately had several flaws. One was in manufacturing and the other two were due to the problems discussed earlier. During manufacturing the company which manufactured the prototypes added a coating to the outside of the helmet shells to make them stronger and to try to prevent the layers from delaminating. The amount of coating that was used on the experimental and control helmet prototypes were different however. This may have an effect on the results of the experiments. Second, support material was used in the manufacture of the experimental

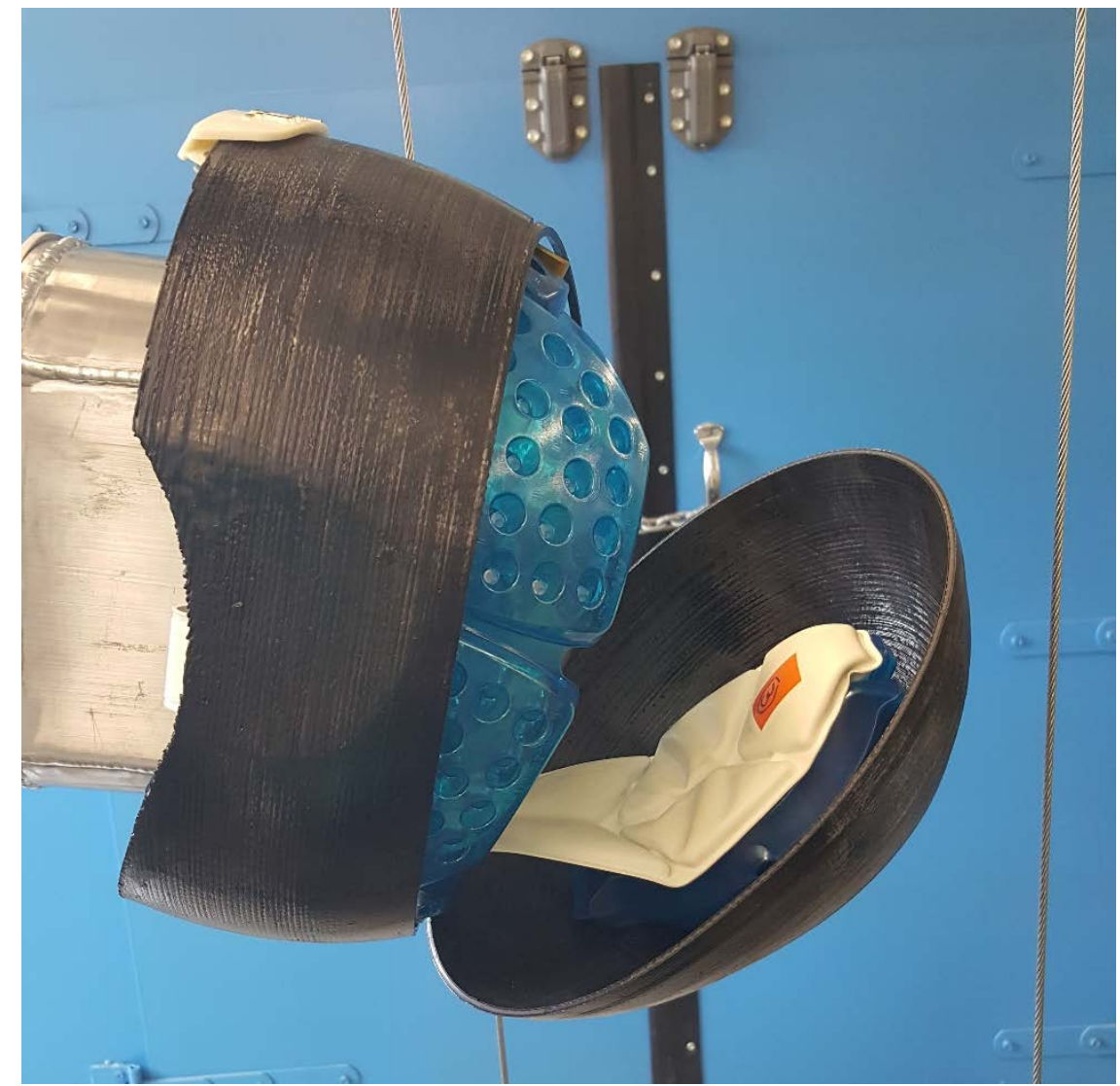

Figure 32. Control helmet after being destroyed by drop testing 
prototype. This support material interfered with the features incorporated into the experimental helmet. Finally, both helmets broke during the impact tests. The helmets peeled apart at the layer boundaries, as seen in Figure 32 for the control helmet. The helmets were able to survive five drops each from 2 feet but both failed when drops were attempted from 4 feet.

\subsection{Drop Tower Observations, Discussion, and Future Work}

After fabrication and assembly was completed on the drop tower, there were several observations and modifications made.

The first observation was that the limit switches were not reliable. Because of the fact that the winch still continues to spool or unspool even after power is removed, the winch would keep moving even though the limits were reached. When the lowering limit switch is reached the winch loses tension and the cable can get tangled. The upper limit switch removes power from the motor when activated, but the lift bar continues another 5 inches. This issue caused the limit switches to not be used for the full scale testing. Since the limit switches, specifically the upper limit switch, were not reliably stopping the system, they were not relied on to provide a consistent drop height. Instead the lift bar was raised to the right height and then the carriage was raised to the lift bar by hand for each drop.

The bolts connecting the hinges to the upper frame were left loose during assembly. This was to allow the cotter pins to be aligned easier. The holes proved to not be perfectly 
inline and a dead-blow mallet is needed to align the holes. Since the pins are taking all the force required to hold the frame in alignment, leaving these bolts loose is not detrimental.

An issue currently present with the drop tower is that the lift bar occasionally is not able to lower smoothly. This is because the lift bar alone does not have enough weight to reliably unspool cable from the winch drum. To combat this, more weight should be added to enable the lift bar to better unspool the cable. Adding weight could help alleviate another issue. The lift bar does not lower horizontal. This does not cause any

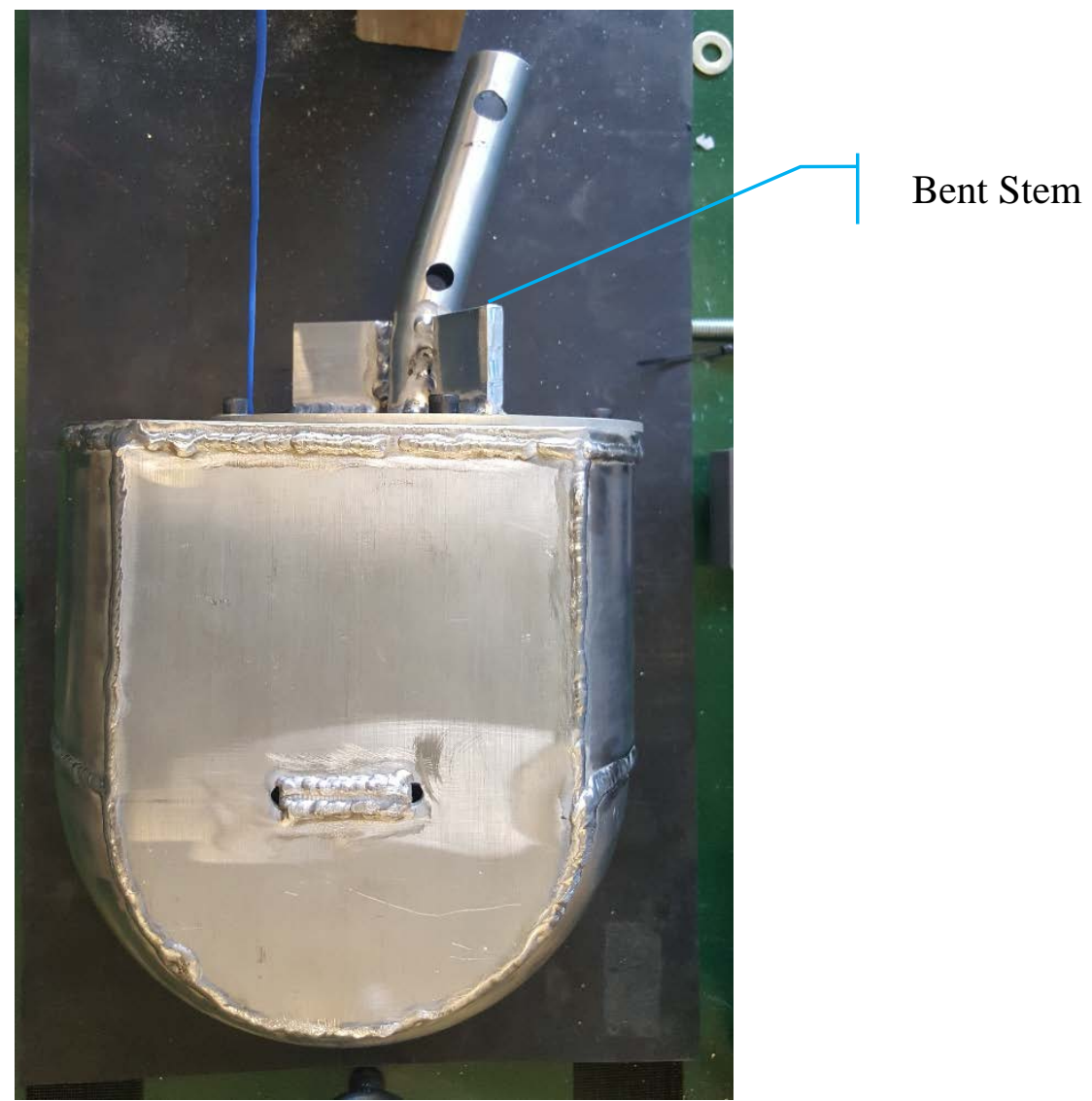

Figure 33. The bent stem attached to the headform 
issues with operation but could be fixed when adding weight to ensure it is balanced on either side of the lift point.

Another issue, only present in the current iteration of the drop tower, is that the mount between the headform and the carriage bent due to the impacts. This is most likely due to the hollow tube used as a stem to replace the actually SIRC designed stem. The bending of the stem may have had an influence on the test results, but no trends were found in the data so it is presumed to not have a noticeable effect. The bent stem can be seen in Figure 33.

To ensure that the tests are more repeatable, the cable tension should be checked. Like earlier stated, checking the cable tension could be accomplished by purchasing a gauge made normally for checking the tension of sailing riggings. The cables for this test were tensioned by feel which could have led to slight errors.

The values that are obtained from the current drop test machine should also be checked against those obtained by outside laboratories for the same helmet. This would allow the machine's value to be trusted more easily. For this specific testing regime it did not matter how the tests compared to tests done on another machine since it was simply comparing two different samples. As long as everything was the same between tests the exact accuracy of the machine is not of great concern. 
To enable full NOCSAE testing to be conducted on the drop tower the headform should be switched to an actual NOCSAE headform. This would enable true NOCSAE tests to be performed and would allow the helmets to fit better on the machine. Additionally, if the correct headform attachment hardware was used the helmets could be impact in all the required spots. The current headform and attachment allows the helmet to be impacted in the front and back but not on the sides.

\subsection{Results and Analysis}

Since the prototype helmets failed during testing, there is not a wealth of data available to analyze. However, a statistical sample was gathered for the 2 foot drop height. 
The data obtained is very different from that of the small scale tests. It required different processing to obtain usable data. The raw data from one of the $2 \mathrm{ft}$ control tests is shown

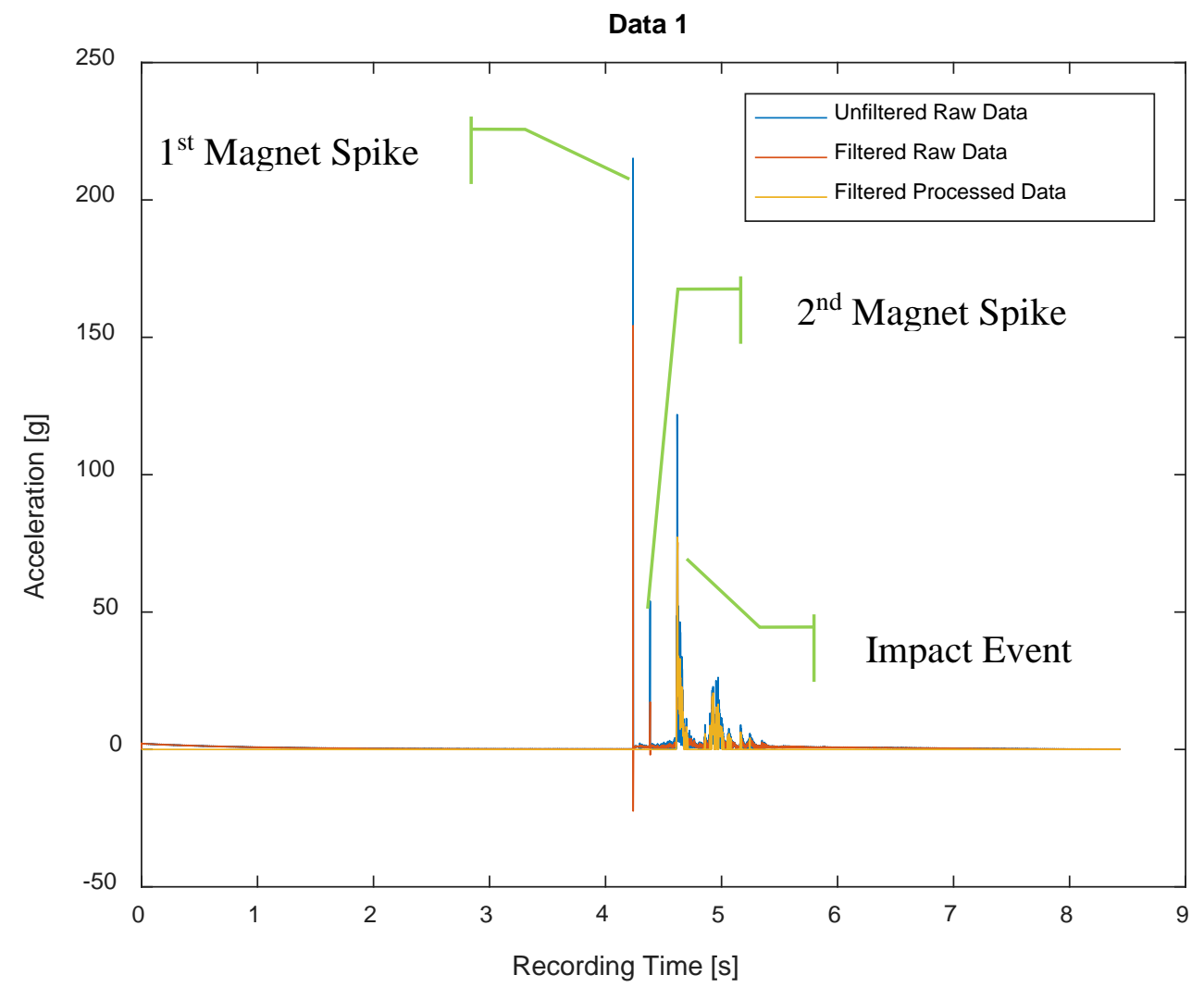

Figure 34. The data from a $2 \mathrm{ft}$ control test

in Figure 34. It can be seen that there are two very high magnitude but short in duration spikes in the beginning of the data. These peaks were discovered to be from the reverse in polarity of the electromagnet release. The second, shorter spike, is from the carriage dropping out of the influence of the electromagnetic field. These two spikes had to be removed from the data in order to find the correct SI value. To remove these spikes the MATLAB program used to analyze the data, which can be found in 
APPENDIX E: Full Scale Testing Analysis Cod, searched for impulse events less than 3 milliseconds and removed them from the data. The graphs were then visually checked to make sure no important spike was removed. The first impulse of actual importance in the data is shown in Figure 35. It can be seen from this plot that the impact event is a lot longer, close to 65 milliseconds, than the small scale tests. This is due to the fact the data is a resultant of multi-axis acceleration. When the headform impacts the impact surface it

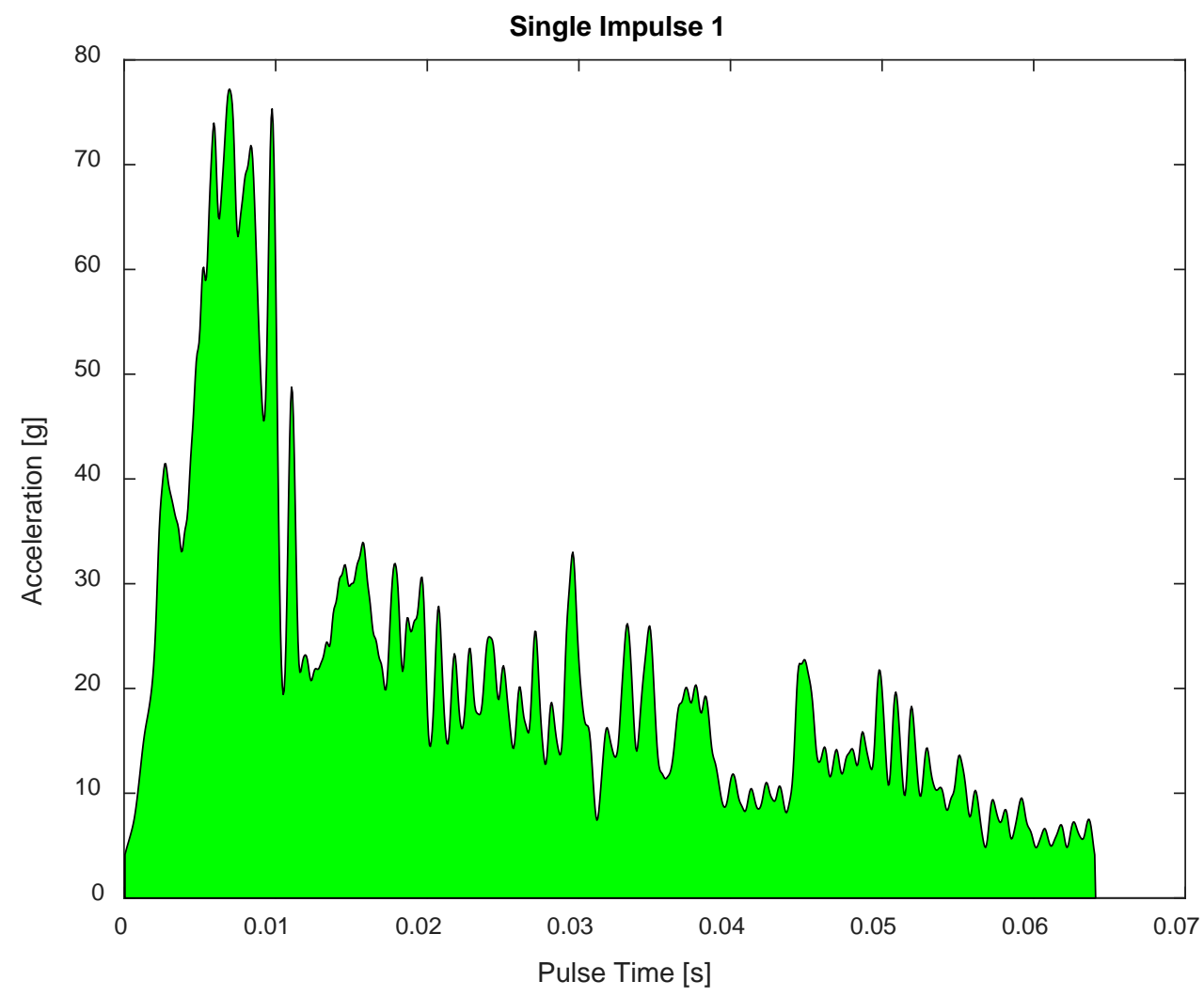

Figure 35. Single impulse (filtered) from full scale control test

undergoes a lot more motion than just the vertical bounce. It shakes back and forth as well as pitches and yaws. All these other aspects of the acceleration add to the acceleration time. In order to cut down on the effects of these other degrees of freedom the data was windowed to the impact time recorded in the high speed camera videos of 
the impacts. These impact times were around 25 milliseconds for the contact between the helmet and the impact pad.

In addition to the windowed and normal SI values it was decided to also use both $\mathrm{HIC}_{36}$ and $\mathrm{HIC}_{15}$, which by definition are also windowed. These values are commonly used in impact tests so they have a lot of credibility in the impact test community. ASTM tests for concussion risk in football helmets by using the max acceleration, to further study the events this criterion was used as well. [11] All the data criteria that were used to analyze the results of the drop tests are shown in Table 5.

Table 5. Results from the full scale testing of the football helmet prototypes

\begin{tabular}{|c|c|c|}
\hline $\begin{array}{c}\text { Concussion Risk } \\
\text { Criteria }\end{array}$ & Control Helmet & $\begin{array}{c}\text { Experimental } \\
\text { Helmet }\end{array}$ \\
\hline $\begin{array}{c}\text { Severity Index } \\
(1200=\text { FAIL })\end{array}$ & 326.90 & 878.52 \\
\hline $\begin{array}{c}\text { Windowed Severity } \\
\text { Index }\end{array}$ & 275.65 & 811.11 \\
\hline HIC 15 & 169.60 & 436.46 \\
$(700=$ FAIL $)$ & 167.85 & 456.13 \\
\hline HIC 36 & $88.92 \mathrm{G}$ & $173.56 \mathrm{G}$ \\
\hline Max Acceleration & & \\
\hline$(300$ G's = FAIL $)$ & & \\
\hline
\end{tabular}


EDC was not used for the full scale tests because of the nature of the data. It was very hard to determine the first two main peaks.

\subsection{Test Specific Conclusions}

The use of full scale prototypes allows us to make a better conclusion about the actual properties and abilities of the experimental design. The results do have to be taken with the same caution as the small scale results; they truly only apply to the current prototypes and an actual helmet may perform differently.

For the small scale testing, statistical tests were used to determine whether the experimental helmets performed better than the control. For the full scale tests statistical tests were not needed because of the great difference in performance between the control and experimental helmets. The results shown for the full scale tests show that the control helmet performs better than the experimental helmet by a factor of 2.5. The five criteria used to study the concussion risk reduction in football helmets all show the same result: the experimental helmet did significantly worse.

Since the five most commonly used impact criteria for impact tests all show the same thing, it is a safe conclusion that the experimental helmet design does not work in the prototype form. The helmet performs worse based on three different organization's criteria. 


\section{CONCLUSIONS}

Now that both the small scale test and the full scale tests are done conclusions can be drawn about the design enhancements imagined by Mr. Bartholomay. While it may seem that the full and small scale results have contradictory results, the differences between the tests can explain that.

With everything known from the full scale tests and the computer modeling conducted separately, the conclusion can be made that the design enhancements provide no reduction to concussion risk compared to a standard football helmet. The design enhancements that were studied in this battery of tests do not seem to work any better than football helmet designs on the market now.

The NOCSAE style drop tower is capable of providing results that are consistent to within a tolerance to be expected from impact events. Impact events, especially those with as many moving parts as a football helmet, have great amounts of error so the tolerance levels of the drop tower are to be expected. The machine still needs to be validated against other laboratories to ensure its accuracy. 


\section{REFERENCES}

[1] B. Burnsed, "A Gray Matter," NCAA Champion, Spring 2015.

[2] B. G. McHenry, "Head Injury Criterion and the ATB," ATB Users' Group, 2004.

[3] NOCSAE, "ND 001-13m15c," 2015.

[4] C. W. Gadd, "Use of a Weighted-Impulse Criterion for Estimating Injury Hazard".

[5] "Helmet FAQ," Snell Memorial Foundation, 2016. [Online]. Available: http://www.smf.org/helmetfaq. [Accessed 20 May 2016].

[6] Xenith, "Xenith Epic," 2016. [Online]. Available: http://www.xenith.com/products/epic-helmet. [Accessed 22 May 2016].

[7] VICIS, "Zero1," 2016. [Online]. Available: http://vicis.co/zero1/. [Accessed 22 May 2016].

[8] NOCSAE, "ND 002-13m15," 2015.

[9] SAE International, "J211-1," 2014.

[10] S. P. Warnert, "Design and Analysis of the Impact Diffusion Helmet Through a Finite Element Analysis Approach," 2016.

[11] ASTM, "F429-10," 2010.

[12] ASTM, "F717-10," 2010.

[13] Schutt Sports, "Vengeance z10," 2016. [Online]. Available: https://www.schuttsports.com/football/vengeance/z10. [Accessed 2 June 2016]. 
APPENDICES

APPENDIX A: DYNATUP FIXTURE ENGINEERING DRAWINGS 


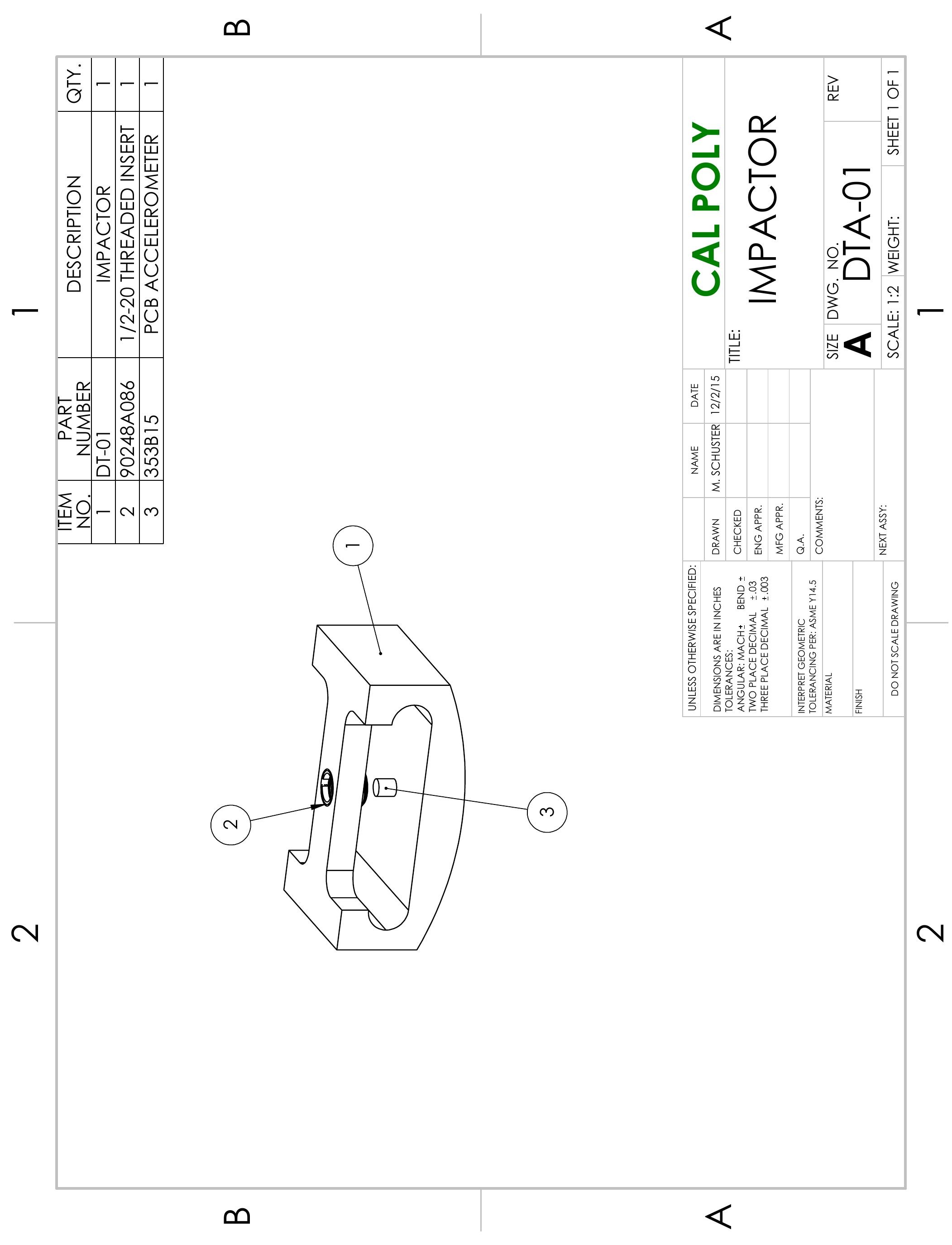




\begin{tabular}{|c|c|c|c|c|c|c|}
\hline$E$ & - & - & - & - & $\nabla$ & 0 \\
\hline 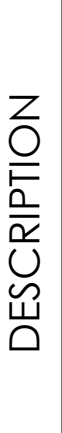 & 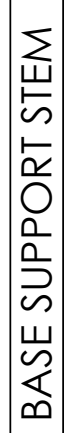 & 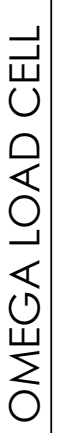 & 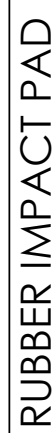 & 足 & 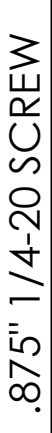 & 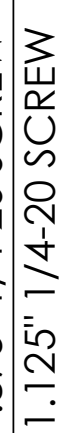 \\
\hline $\begin{array}{l}\bar{z} \\
\frac{\alpha}{\alpha} \\
\frac{\alpha}{\alpha}\end{array}$ & $\begin{array}{l}\mathfrak{\gamma} \\
0 \\
1 \\
\vdots \\
0\end{array}$ & 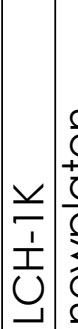 & 它 & & 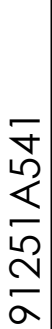 & $\begin{array}{ll}5 \\
4 \\
4 \\
5 \\
2\end{array}$ \\
\hline ○ & & & & & 0 & \\
\hline
\end{tabular}
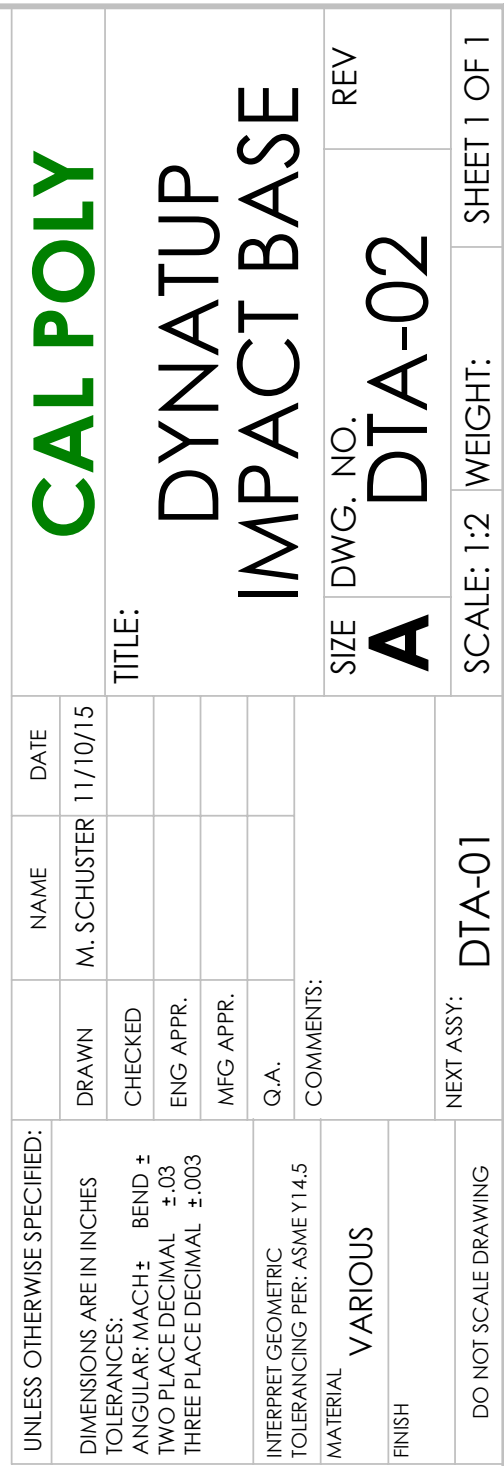

N

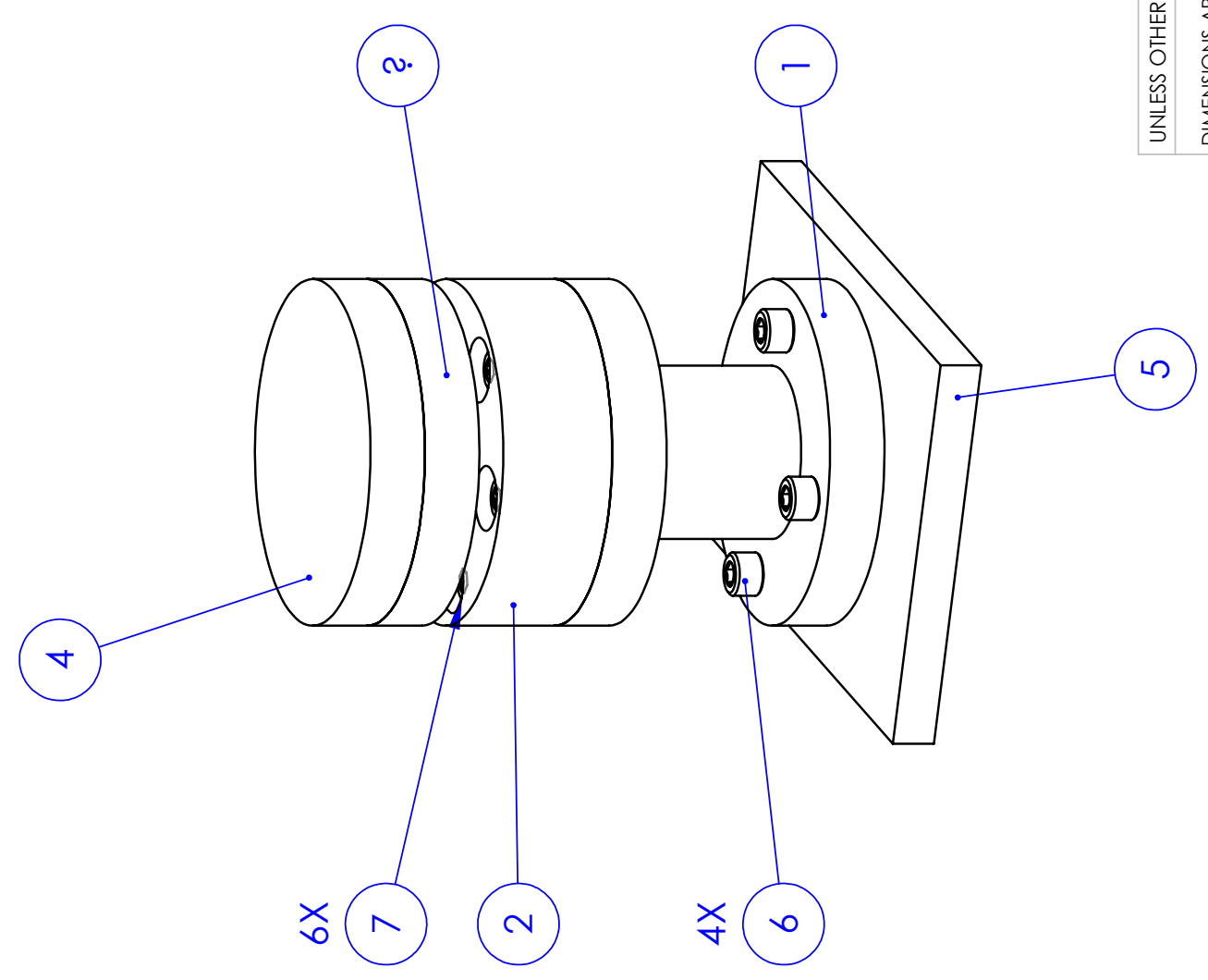




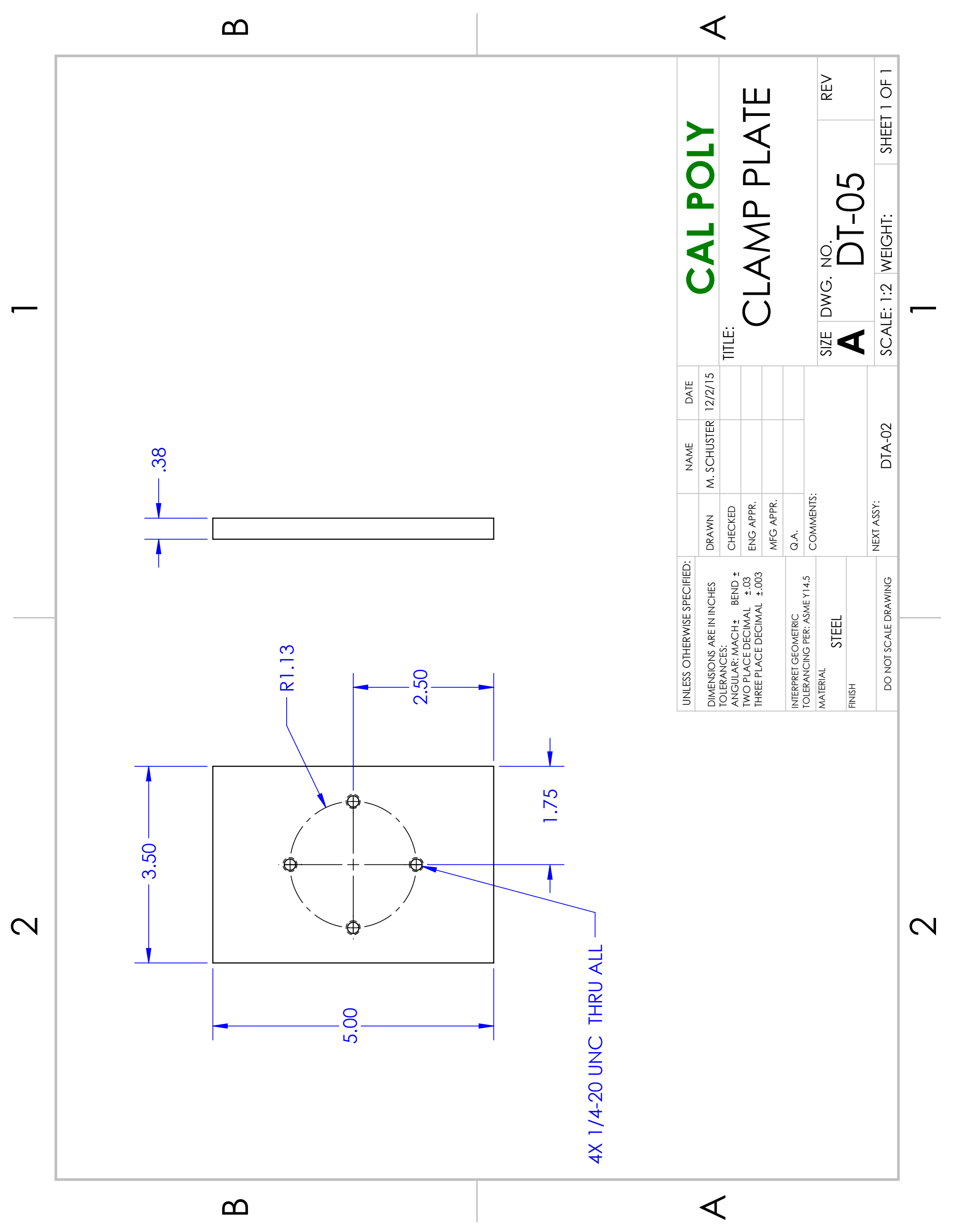



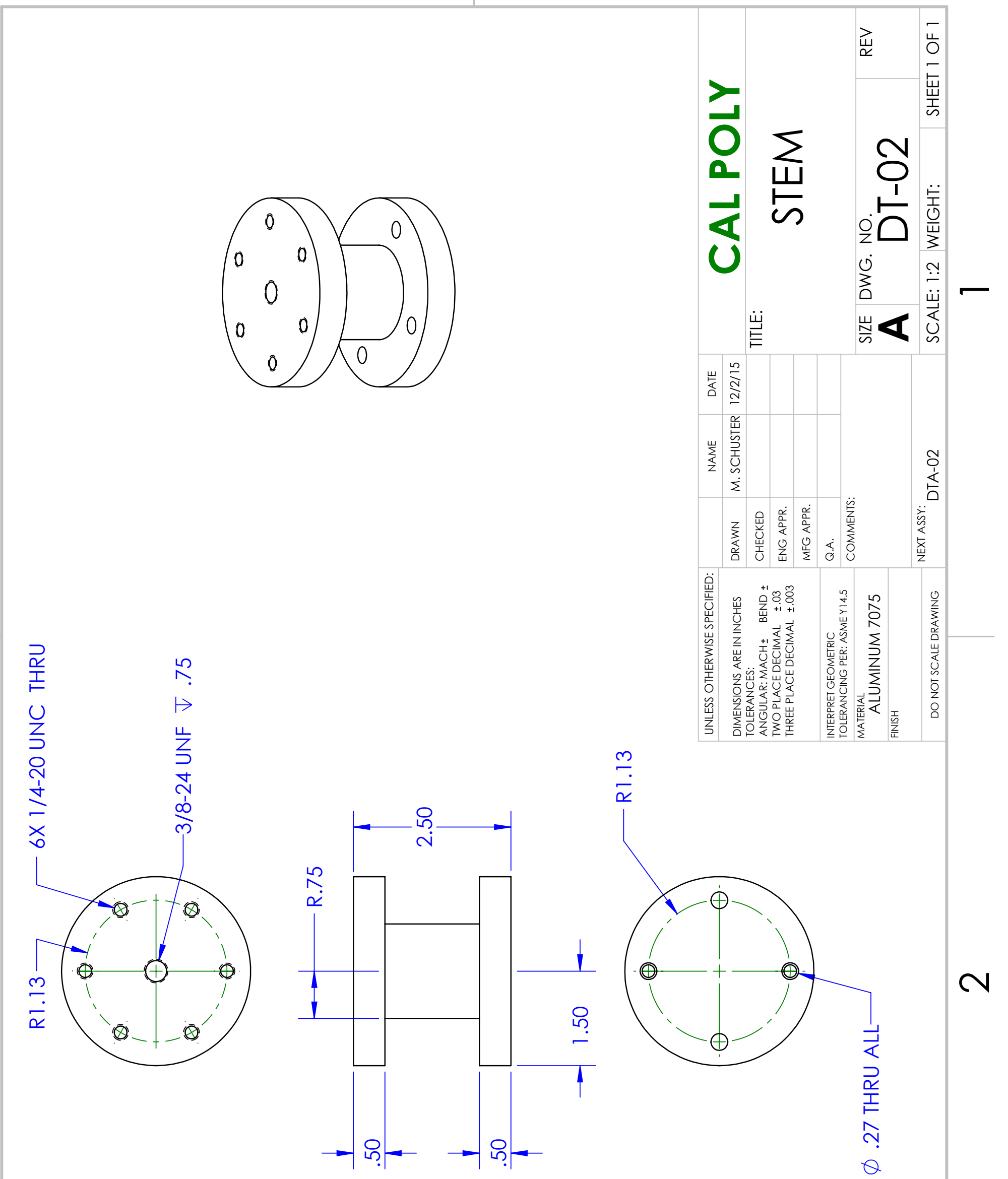

N

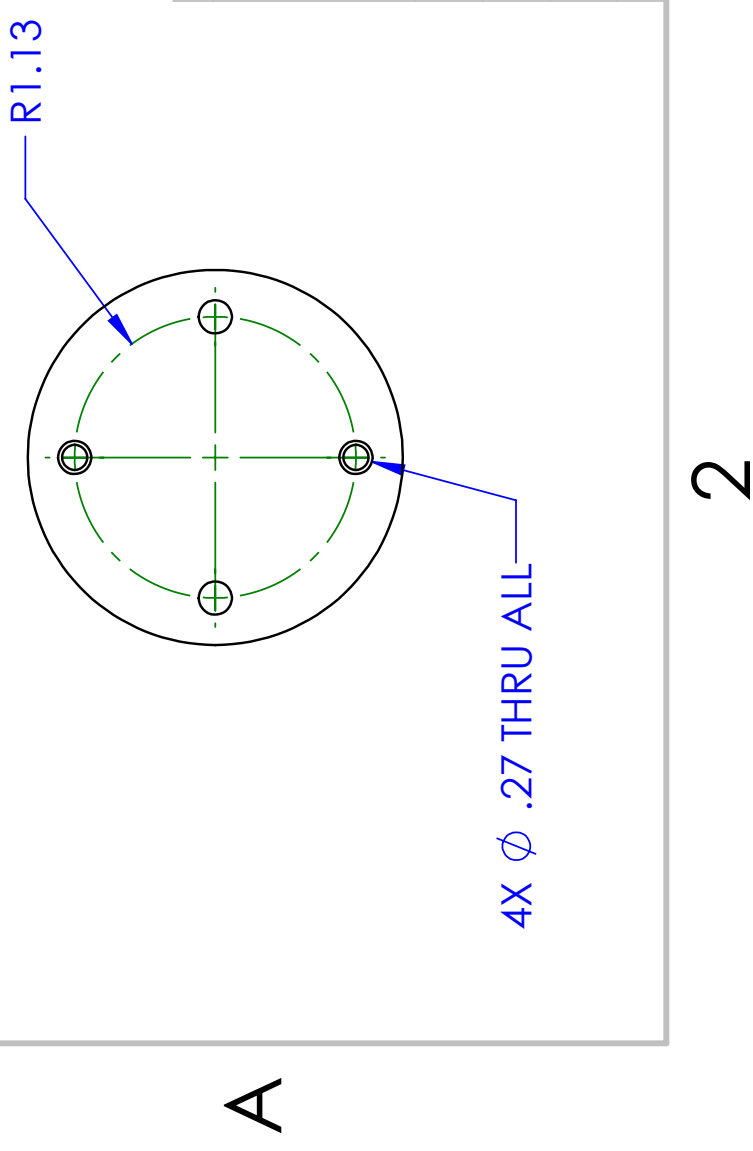




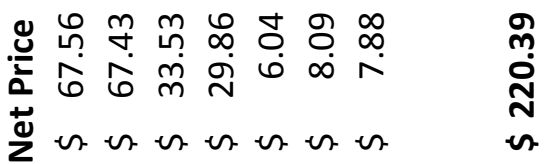

ษ

능

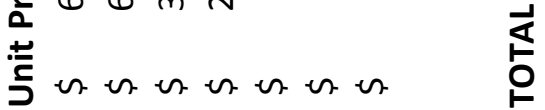

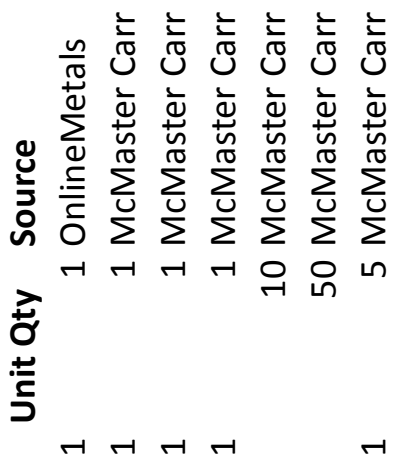

¿
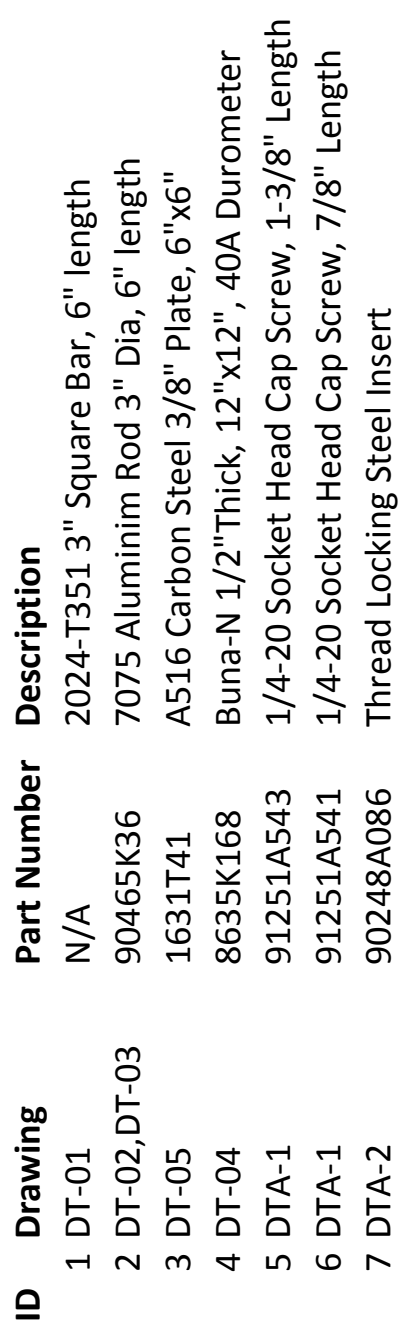
APPENDIX B: SMALL SCALE TESTING ANALYSIS MATLAB CODE 


\section{Table of Contents}

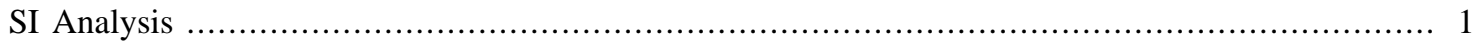

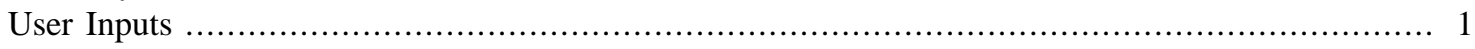

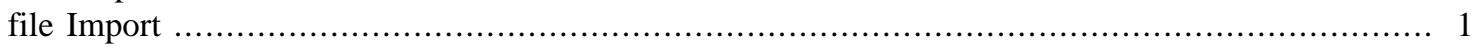

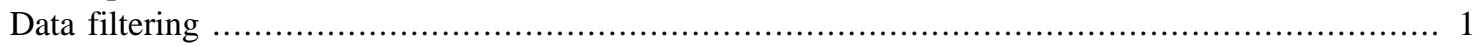

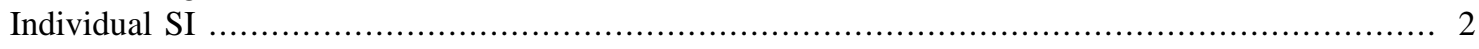

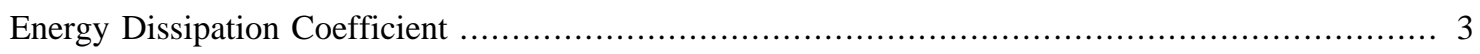

\section{SI Analysis}

Michael Schuster 2/18/2016

analyses data to determine SI

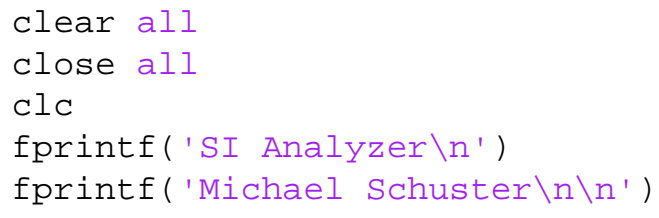

\section{User Inputs}

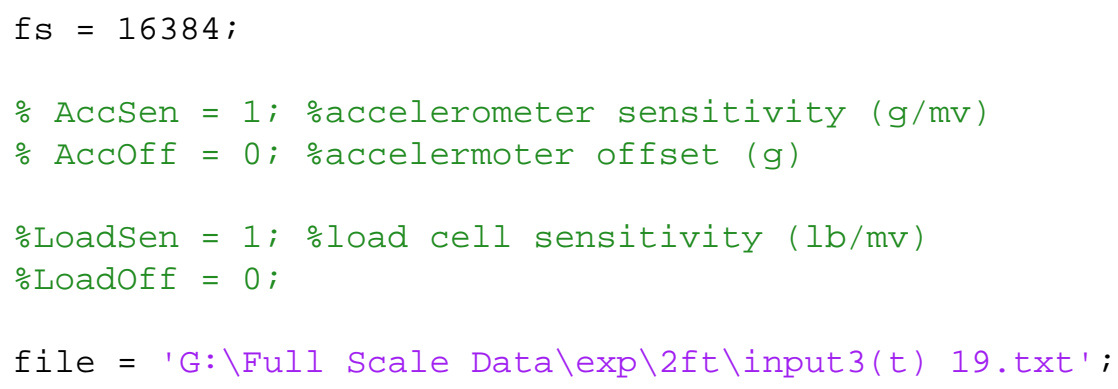

\section{file Import}

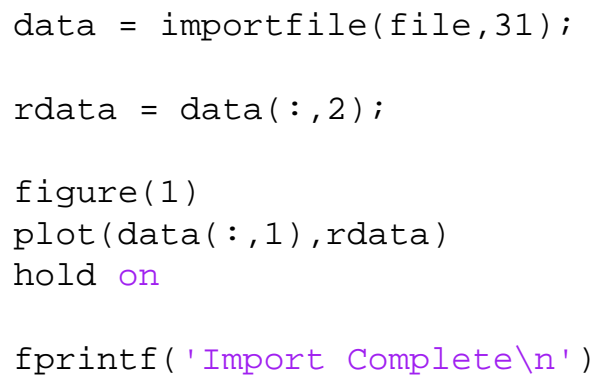

\section{Data filtering}

fdata $=$ butter16(rdata); 
plot (data $(:, 1)$, fdata)

fprintf('Filtering Complete $\backslash n \backslash n$ ')

\section{Individual SI}

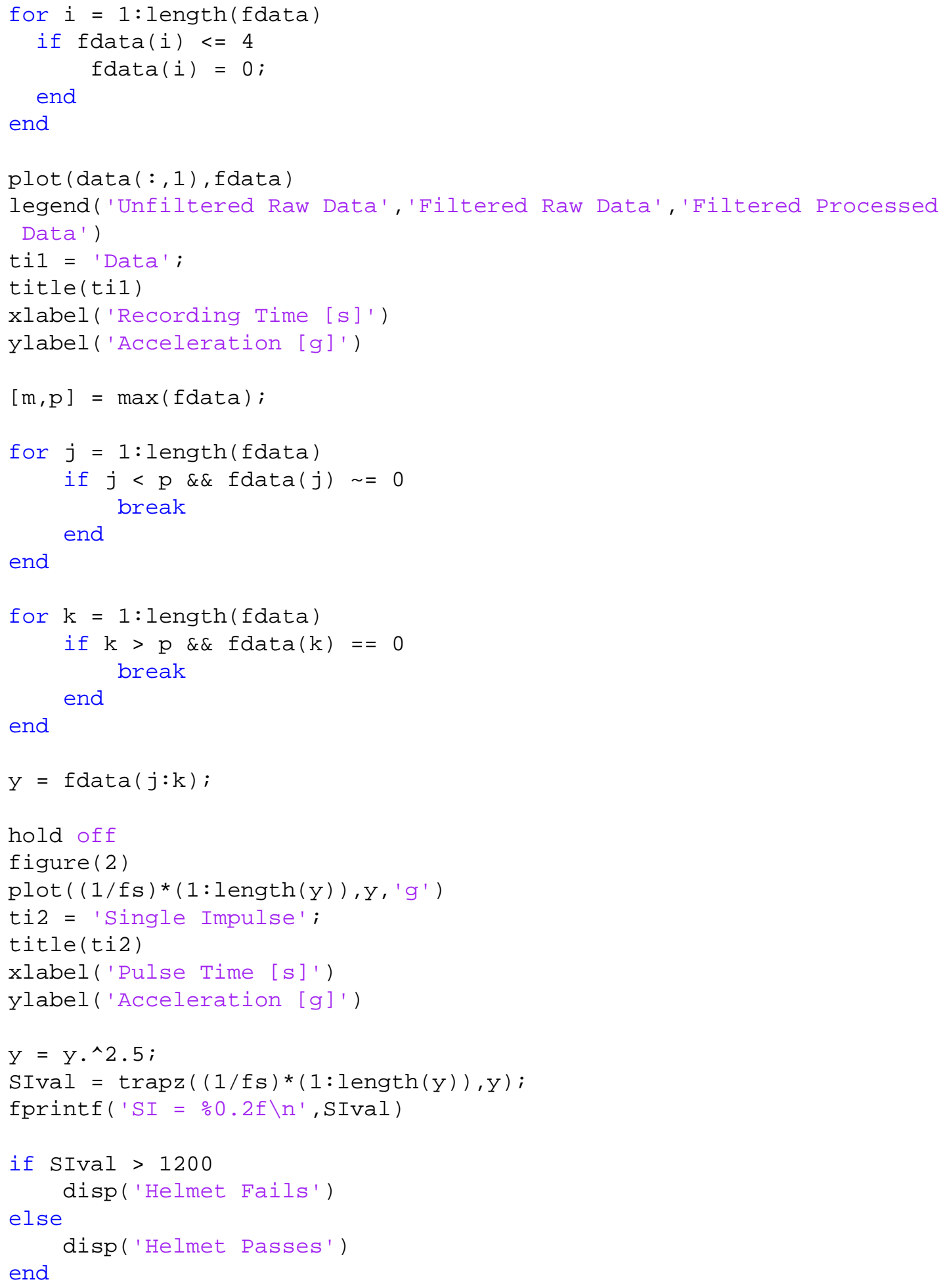




\section{Energy Dissipation Coefficient}

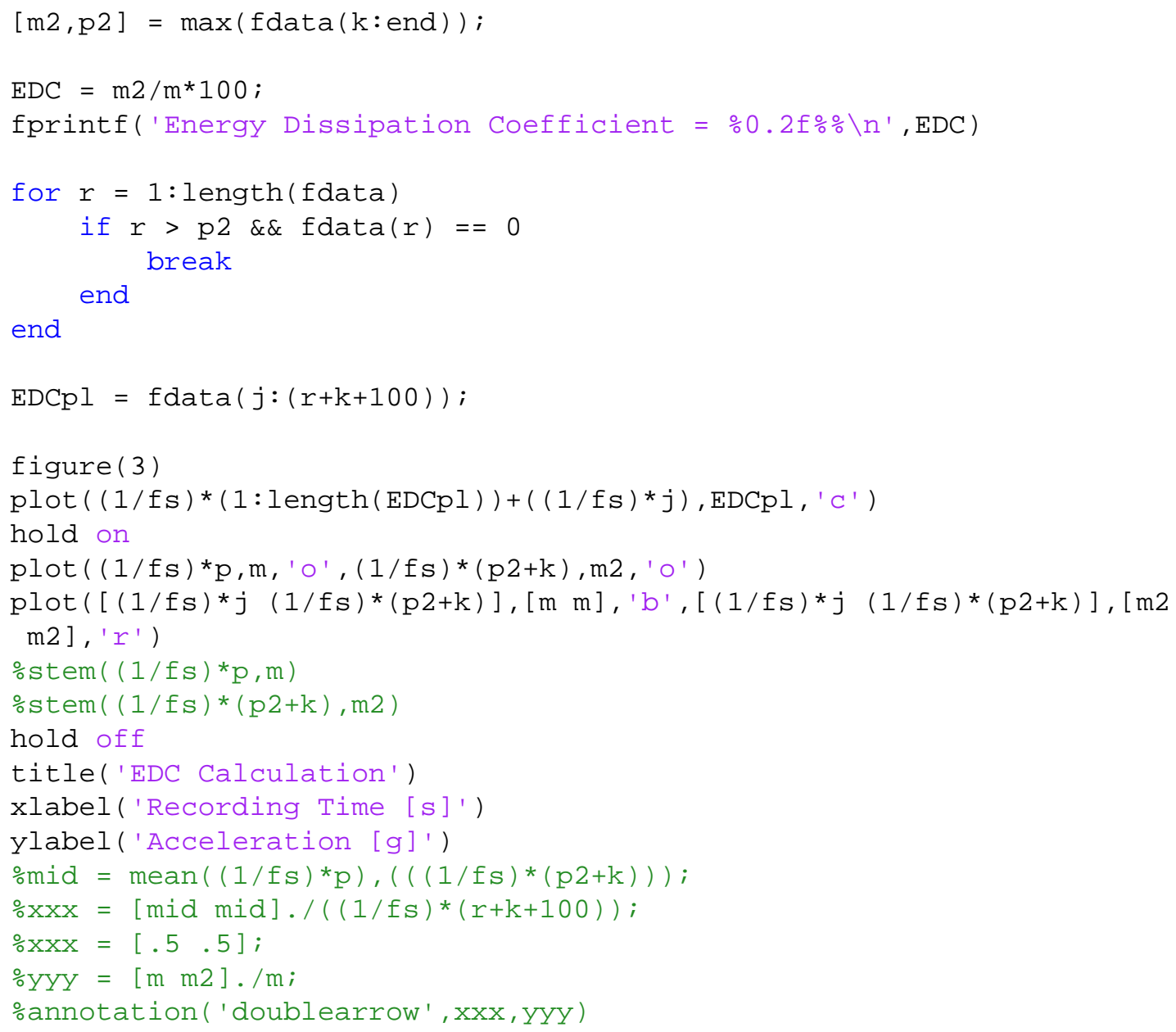

Published with MATLAB® R2016a 


\section{Table of Contents}

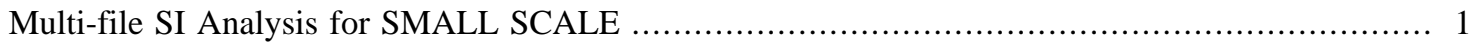

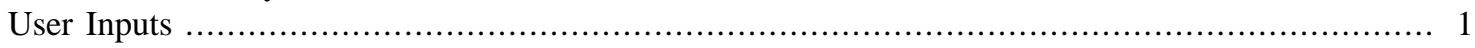

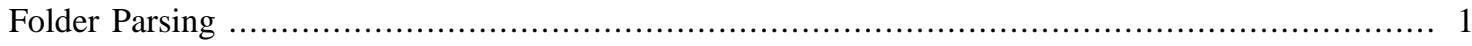

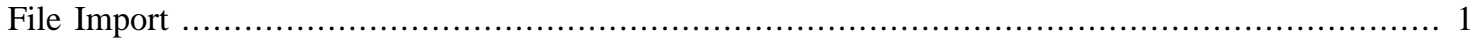

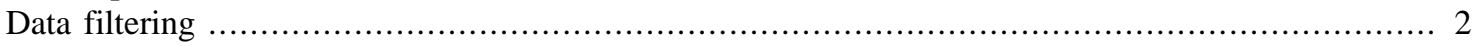

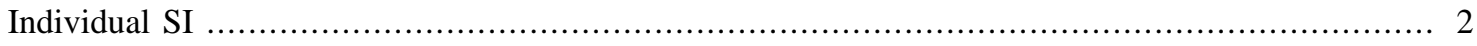

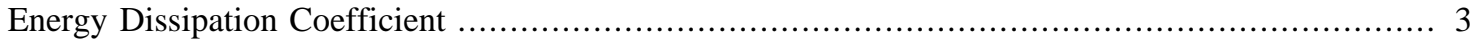

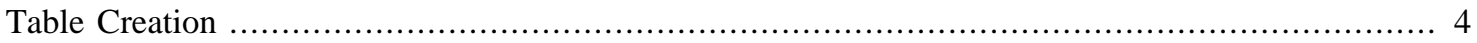

\section{Multi-file SI Analysis for SMALL SCALE}

Michael Schuster 2/18/2016

analyses data to determine SI

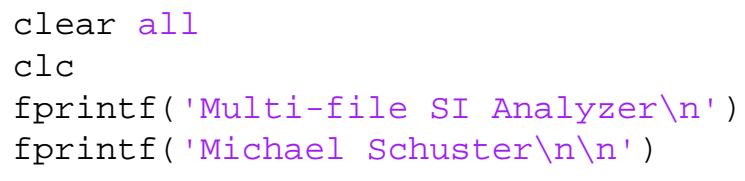

\section{User Inputs}

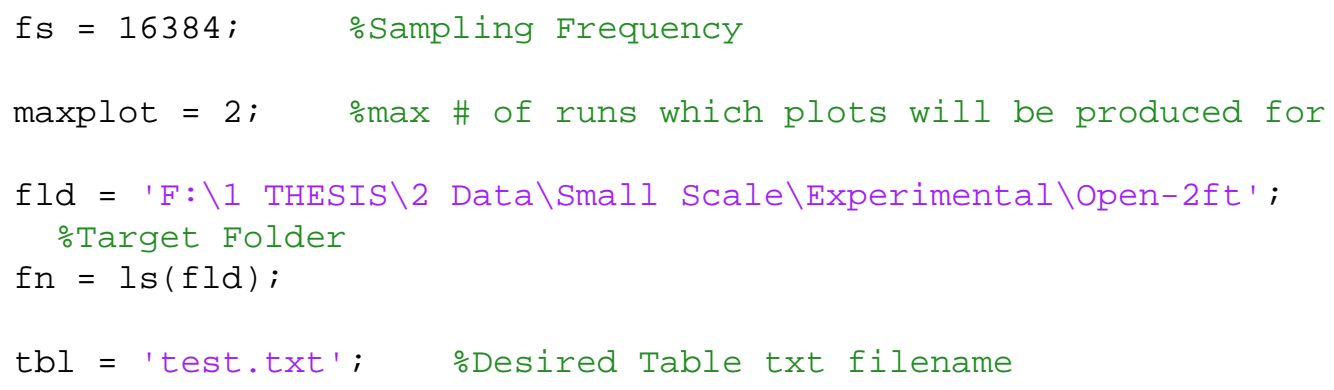

\section{Folder Parsing}

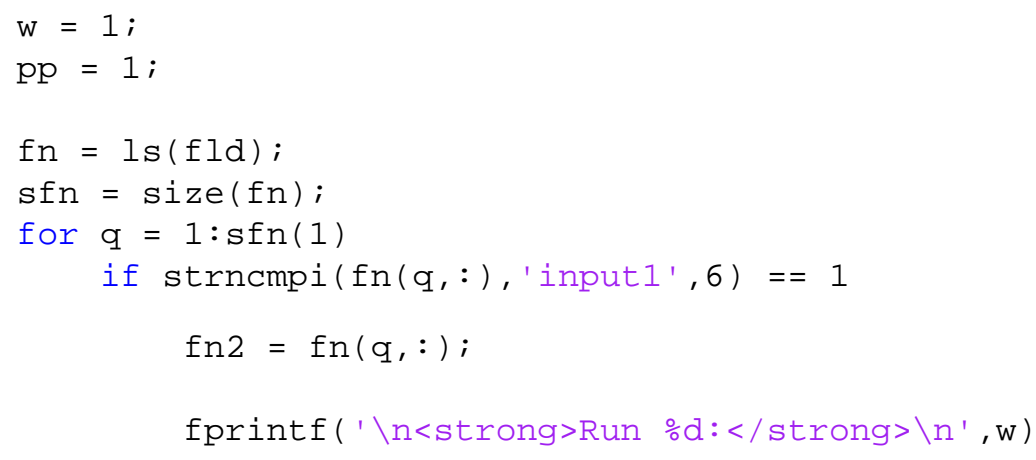

File Import

$$
\text { data }=\text { importfile }\left(\operatorname{strcat}\left(f l d, '^{\prime}, \text { fn2 }\right), 31\right) \text {; }
$$




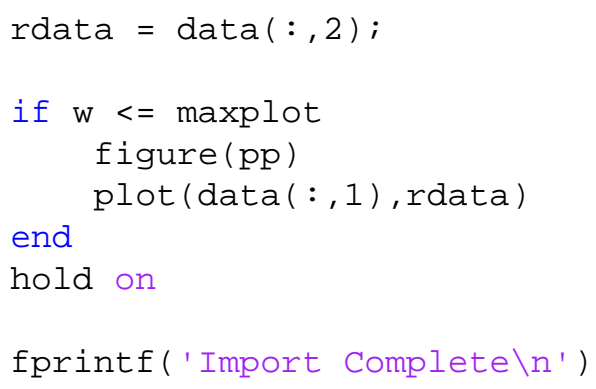

\section{Data filtering}

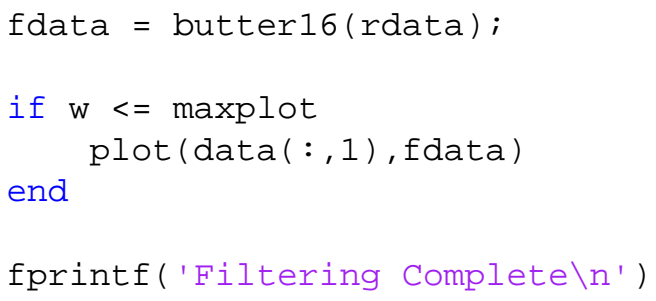

\section{Individual SI}

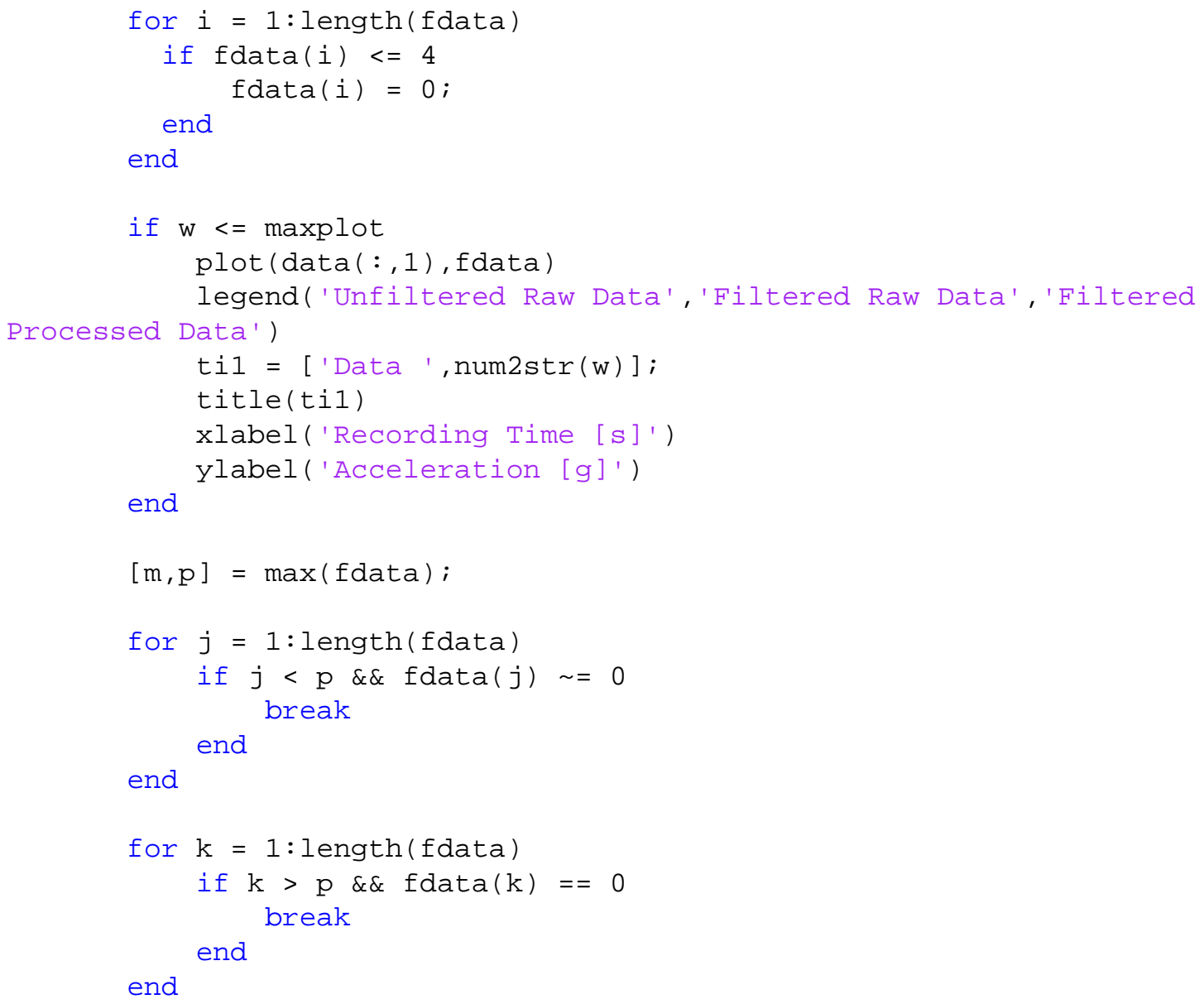




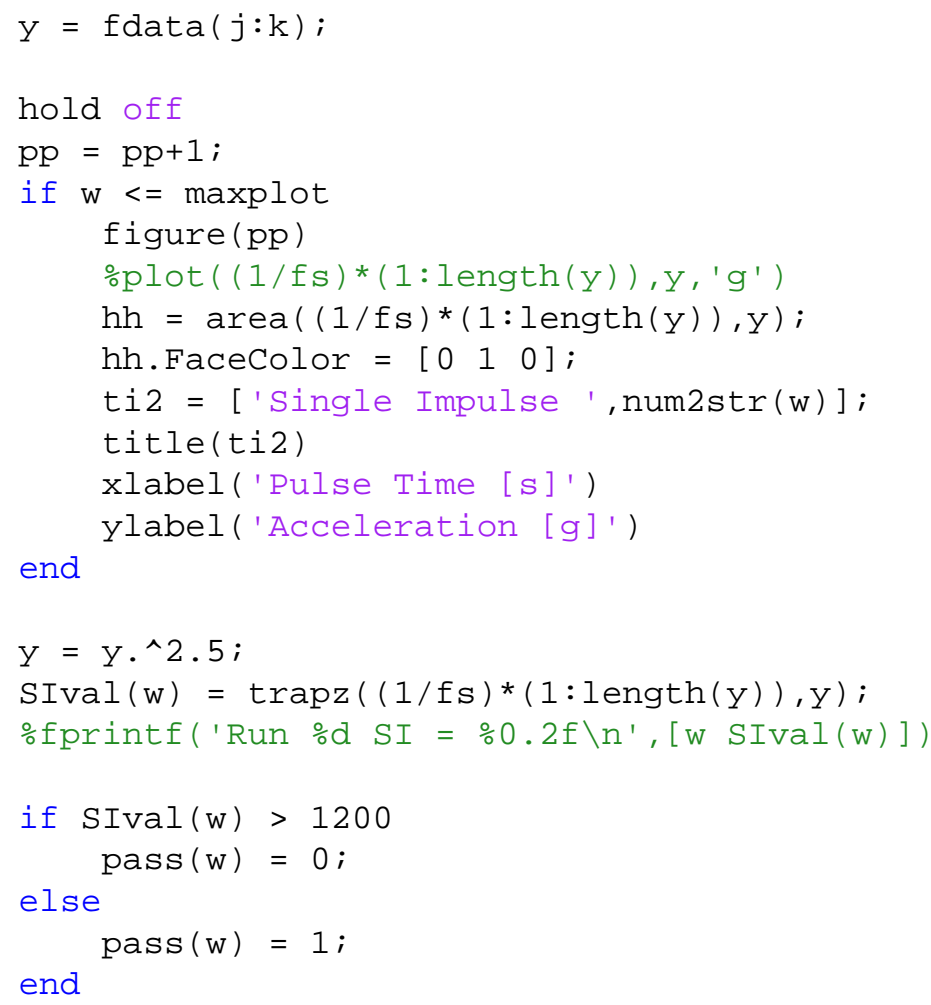

\section{Energy Dissipation Coefficient}

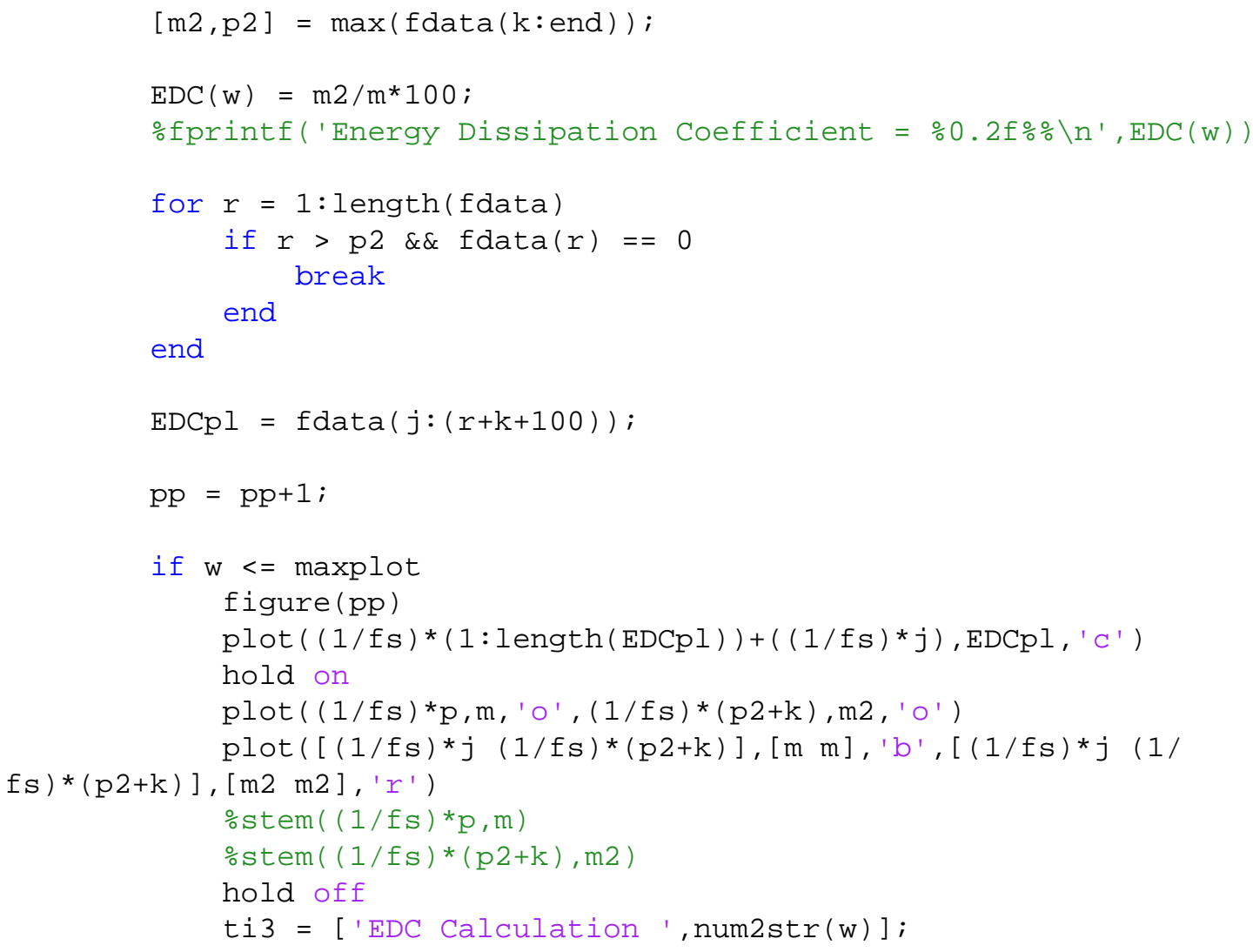




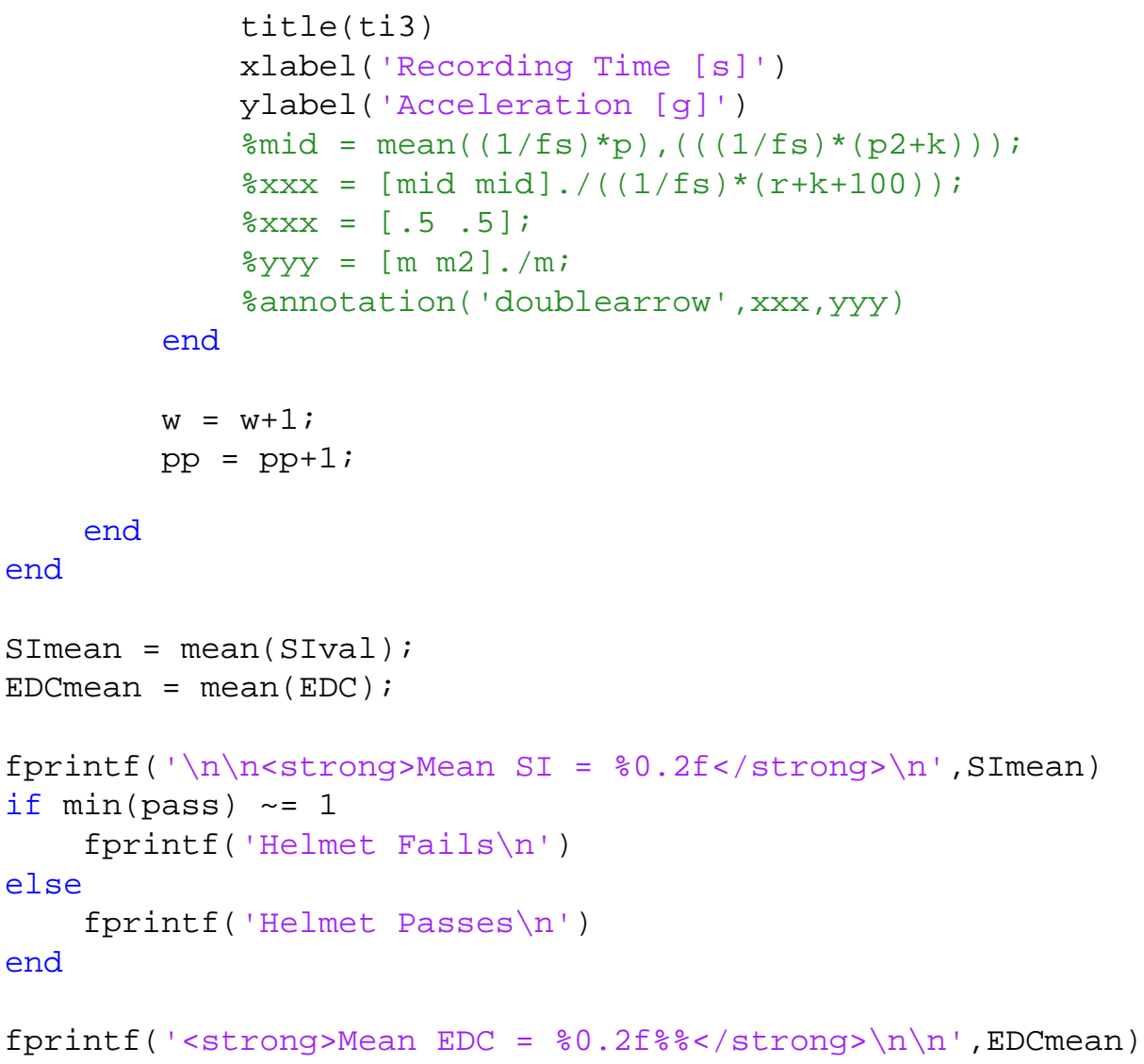

\section{Table Creation}

$\mathrm{T}=$ table([1:(w-1) ] ', SIval',EDC', 'VariableNames', \{'Run', 'SI', 'EDC'\}); disp ( T)

writetable $(\mathrm{T}, \mathrm{tbl})$

disp ('DONE')

Published with MATLAB® R2016a 


\title{
Table of Contents
}

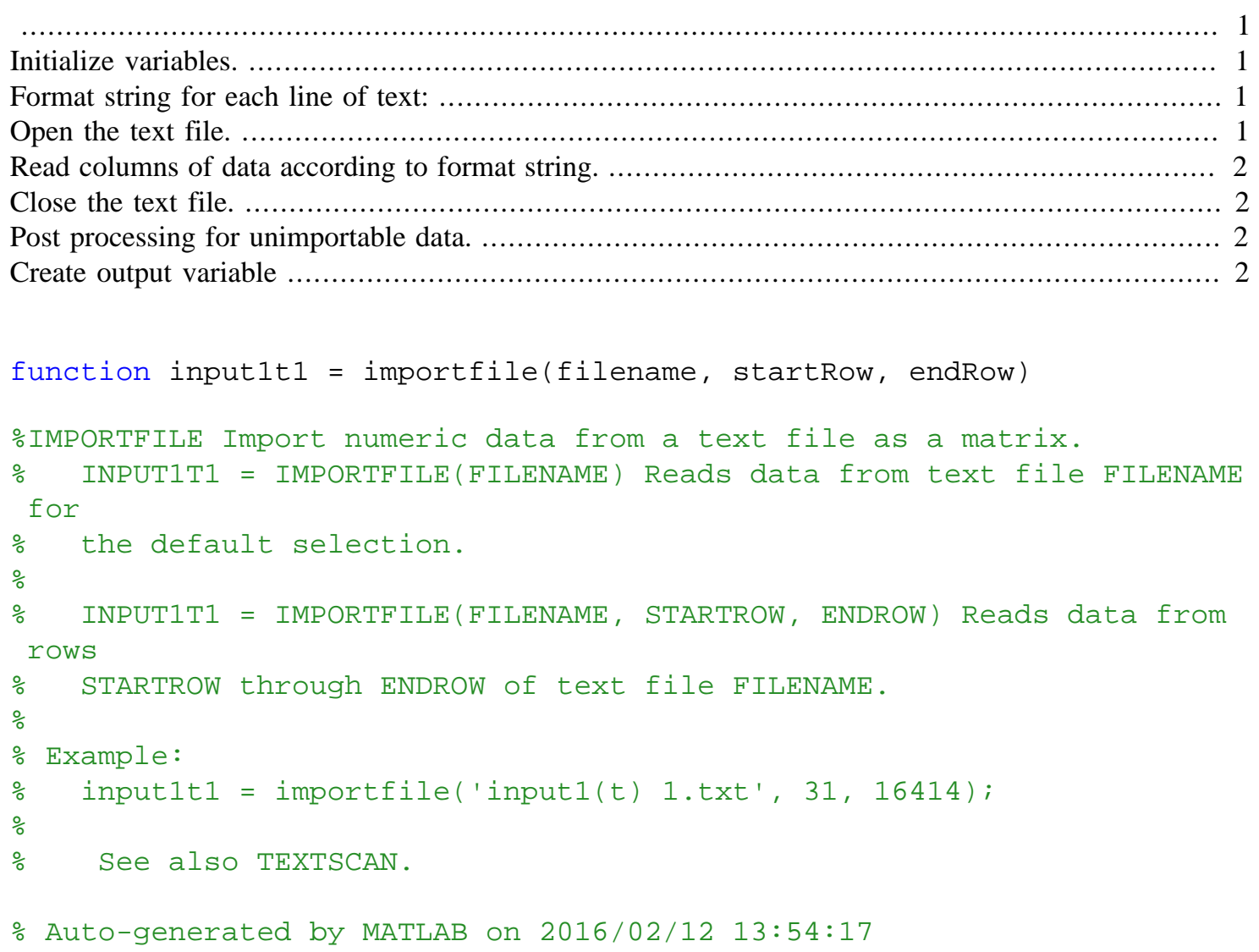

\section{Initialize variables.}

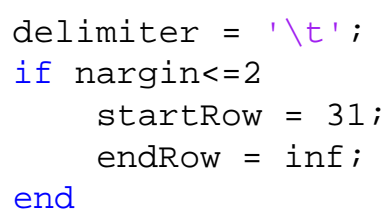

\section{Format string for each line of text:}

\author{
column 1 : double $(\% f)$ \\ \% column2: double (\%f) \\ \% For more information, see the TEXTSCAN documentation. \\ formatSpec $\left.={ }^{\prime} \circ \mathrm{f} \% \mathrm{f} \%\left[{ }^{\wedge} \backslash \mathrm{n} \backslash \mathrm{r}\right]\right]^{\prime}$;
}

\section{Open the text file.}

fileID = fopen(filename, 'r'); 


\section{Read columns of data according to format string.}

This call is based on the structure of the file used to generate this code. If an error occurs for a different file, try regenerating the code from the Import Tool.

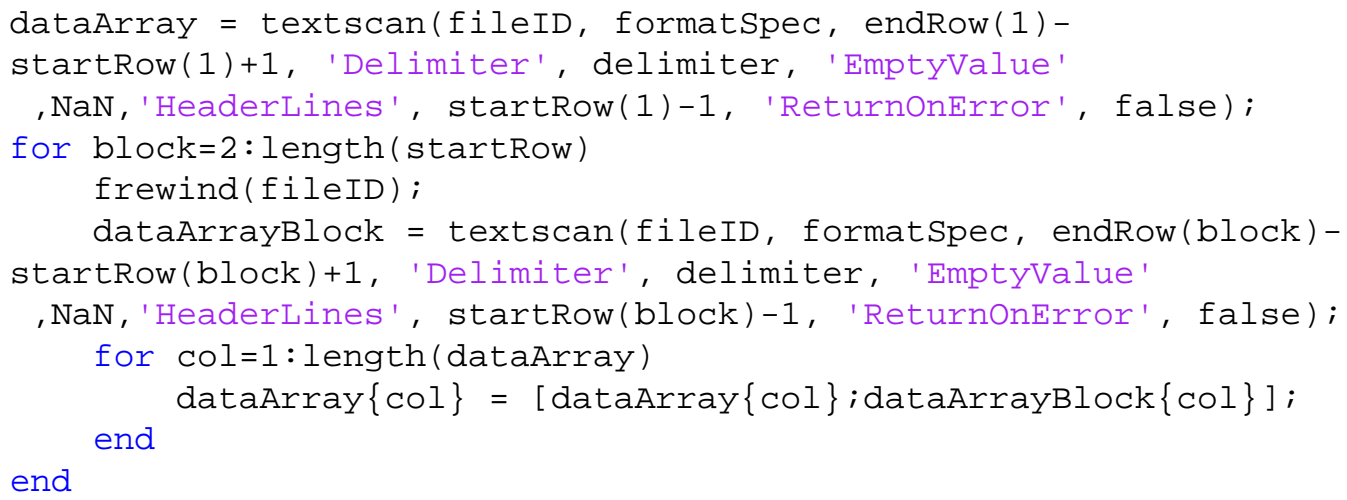

Close the text file.

fclose (fileID);

\section{Post processing for unimportable data.}

No unimportable data rules were applied during the import, so no post processing code is included. To generate code which works for unimportable data, select unimportable cells in a file and regenerate the script.

\section{Create output variable}

input 1 t $1=[$ dataArray $\{1:$ end-1 $\}]$;

Published with MATLAB® R2016a 


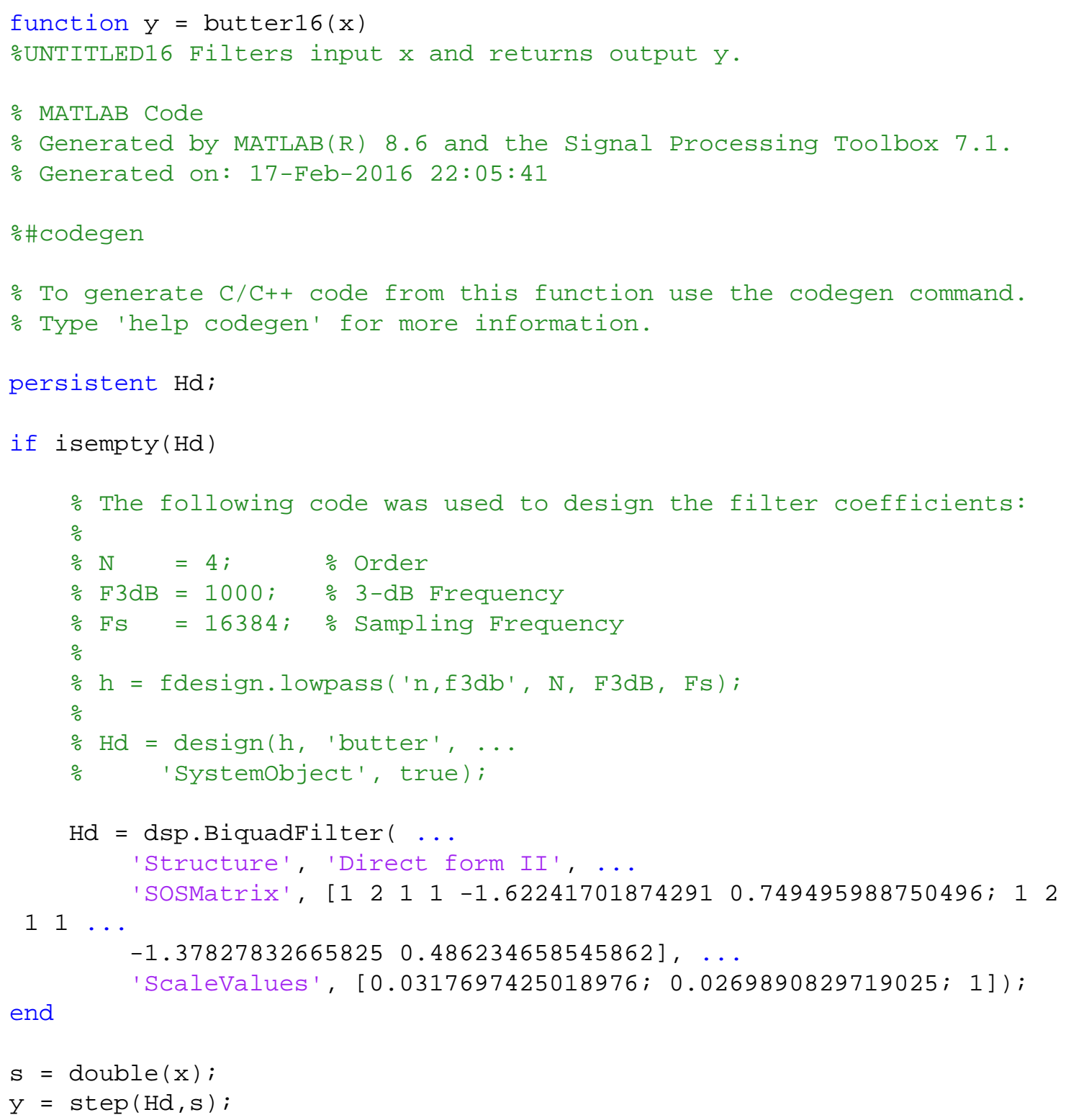

Published with MATLAB® R2016a 
APPENDIX C: SIRC DROP TOWER DRAWINGS 


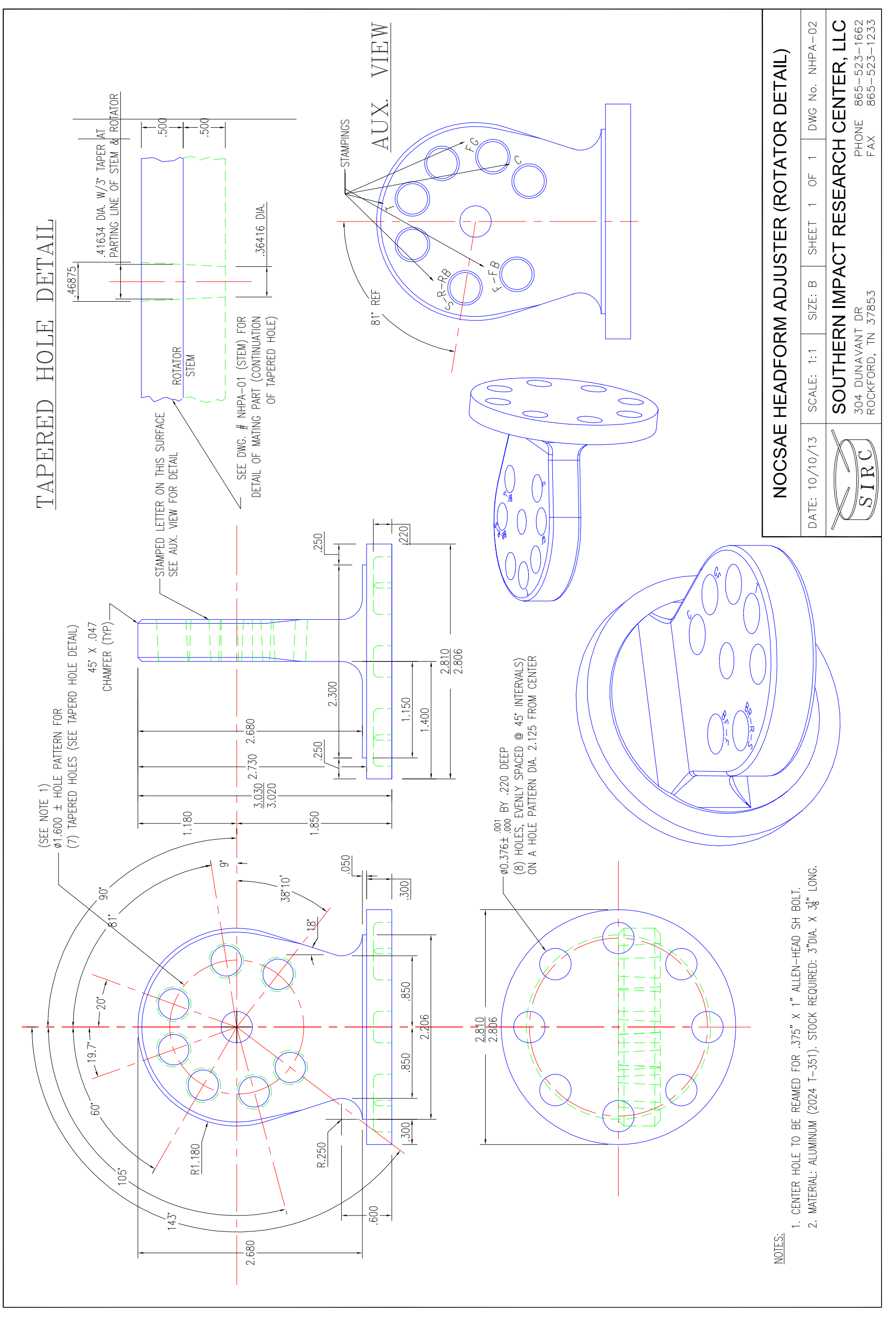



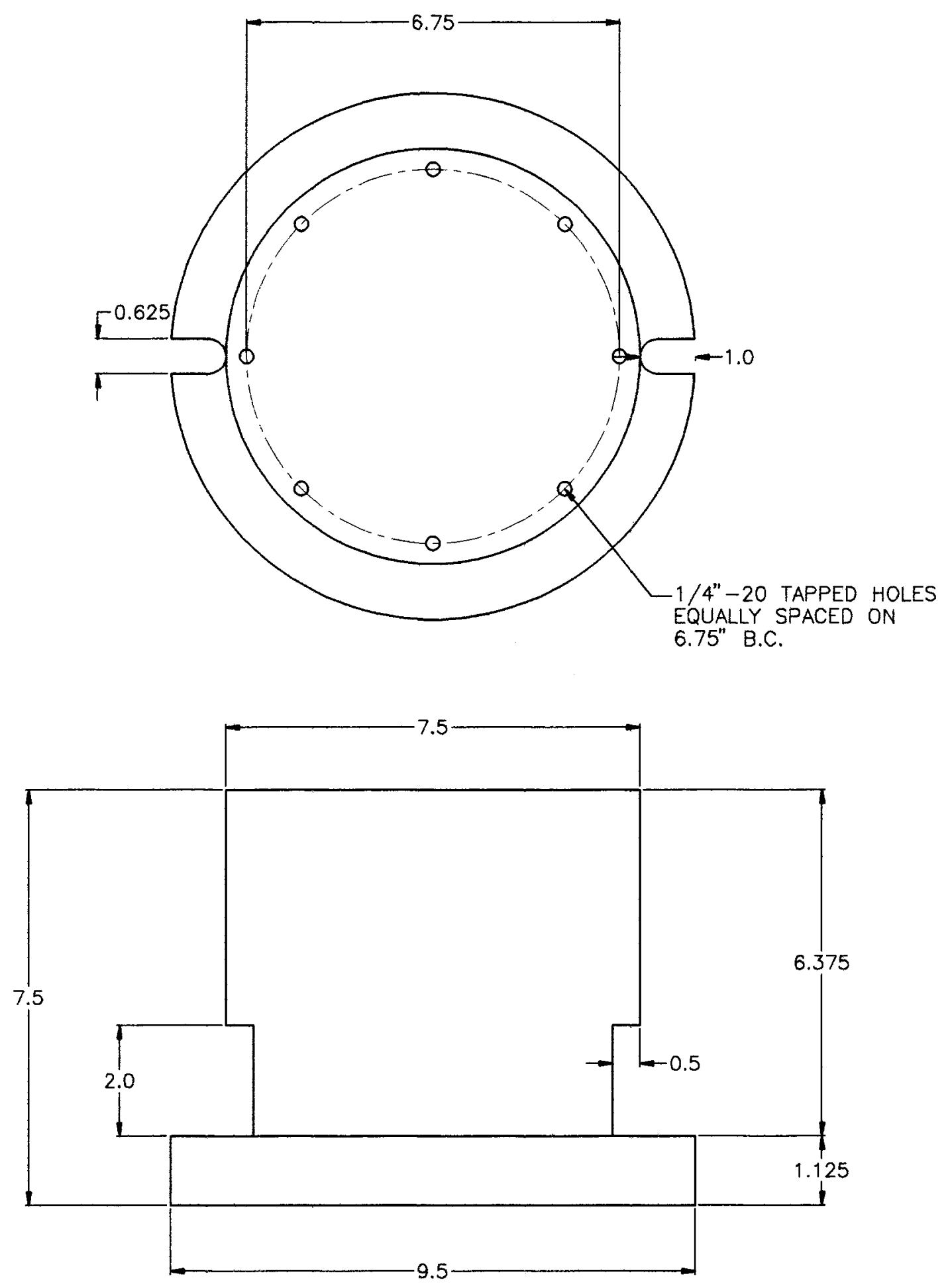

NOTES:

MATERIAL: STEEL $10^{\prime \prime}$ DIA. $\times 7.5^{\prime \prime}$ LONG

TEST ANVIL

\begin{tabular}{|c|c|c|c|}
\hline $2-14-98$ & SCALE: $1: 3$ & SHEET 1 OF 1 & DRAWING: TA-02 \\
\hline
\end{tabular}




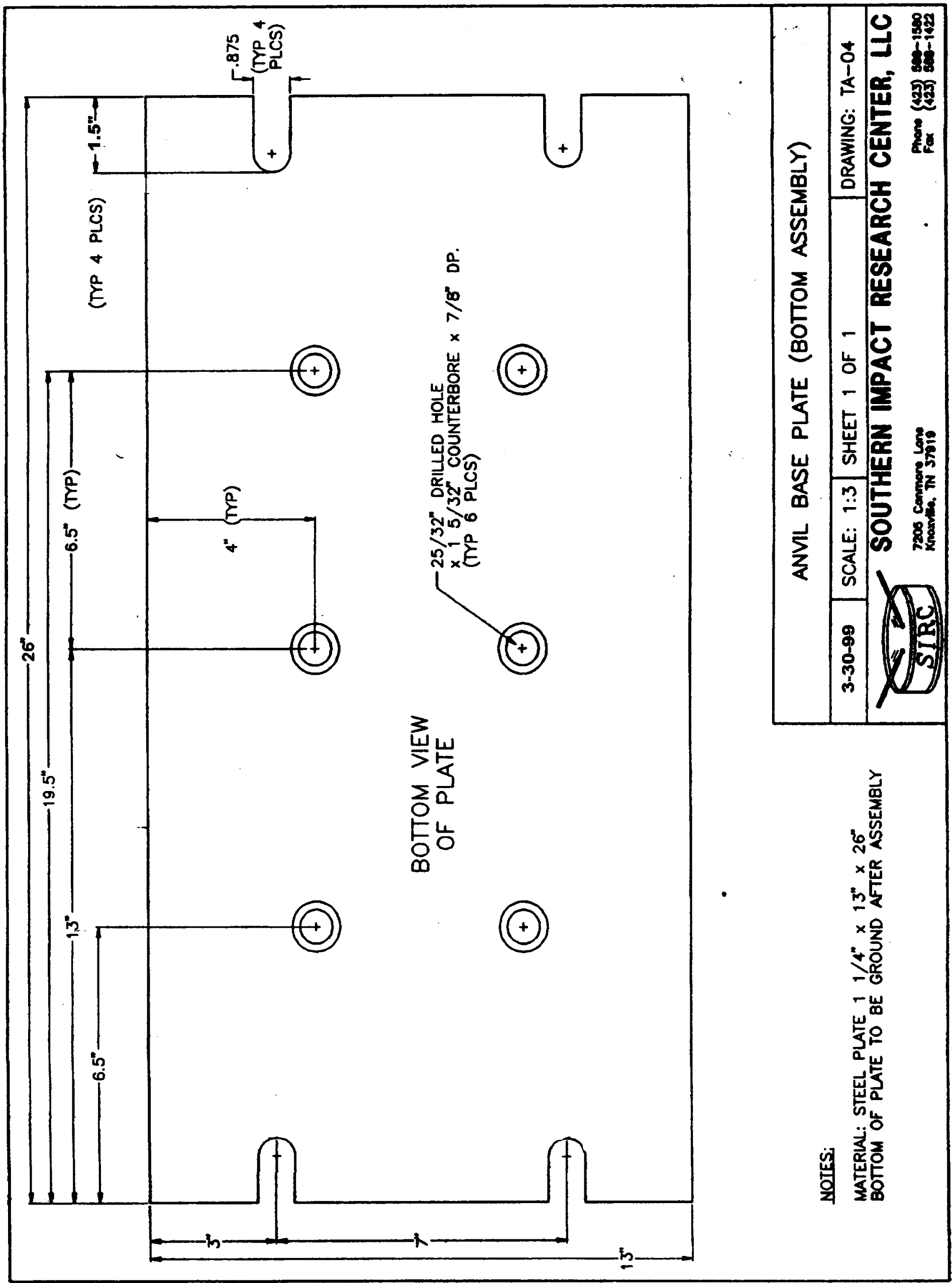




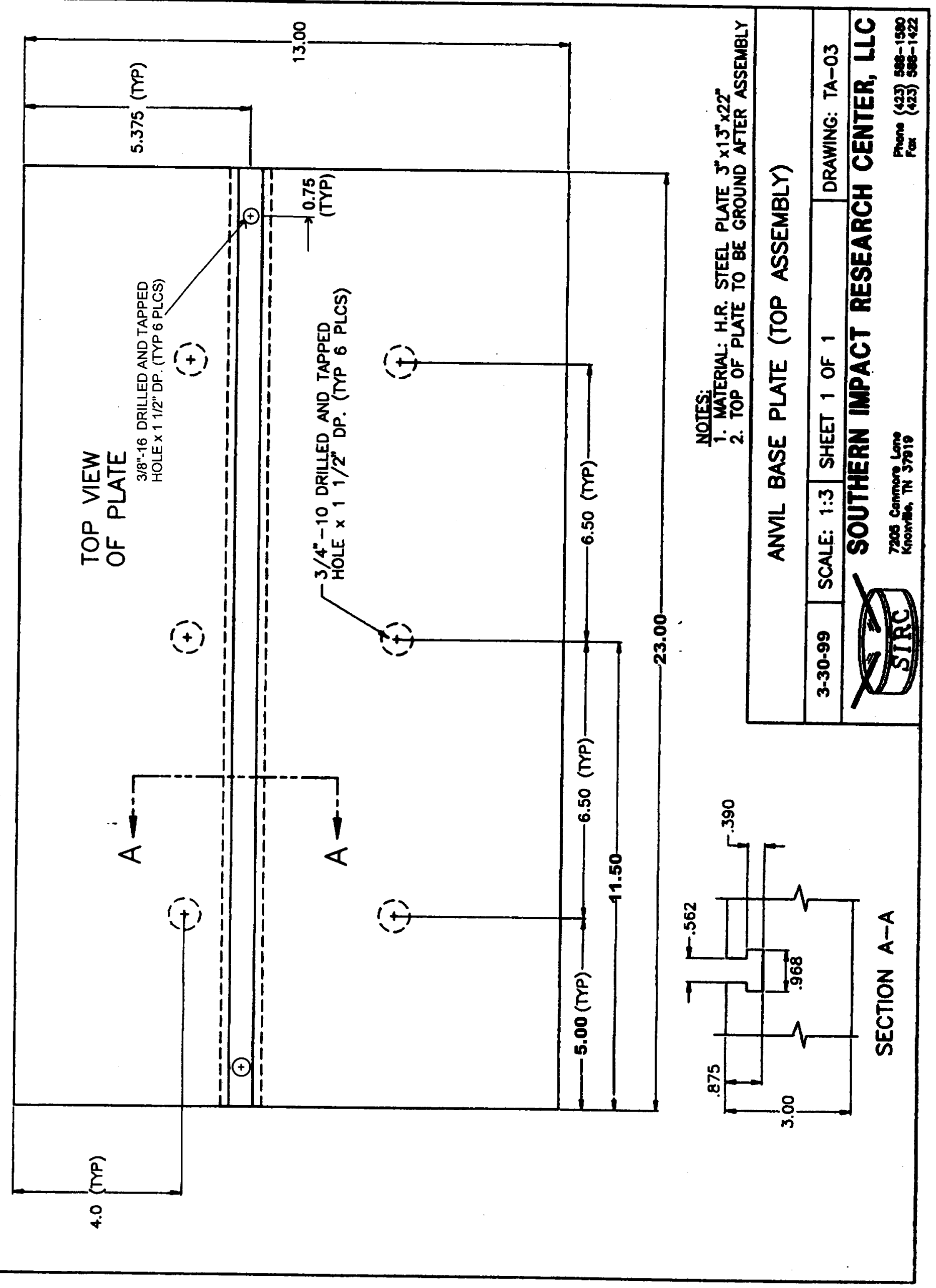



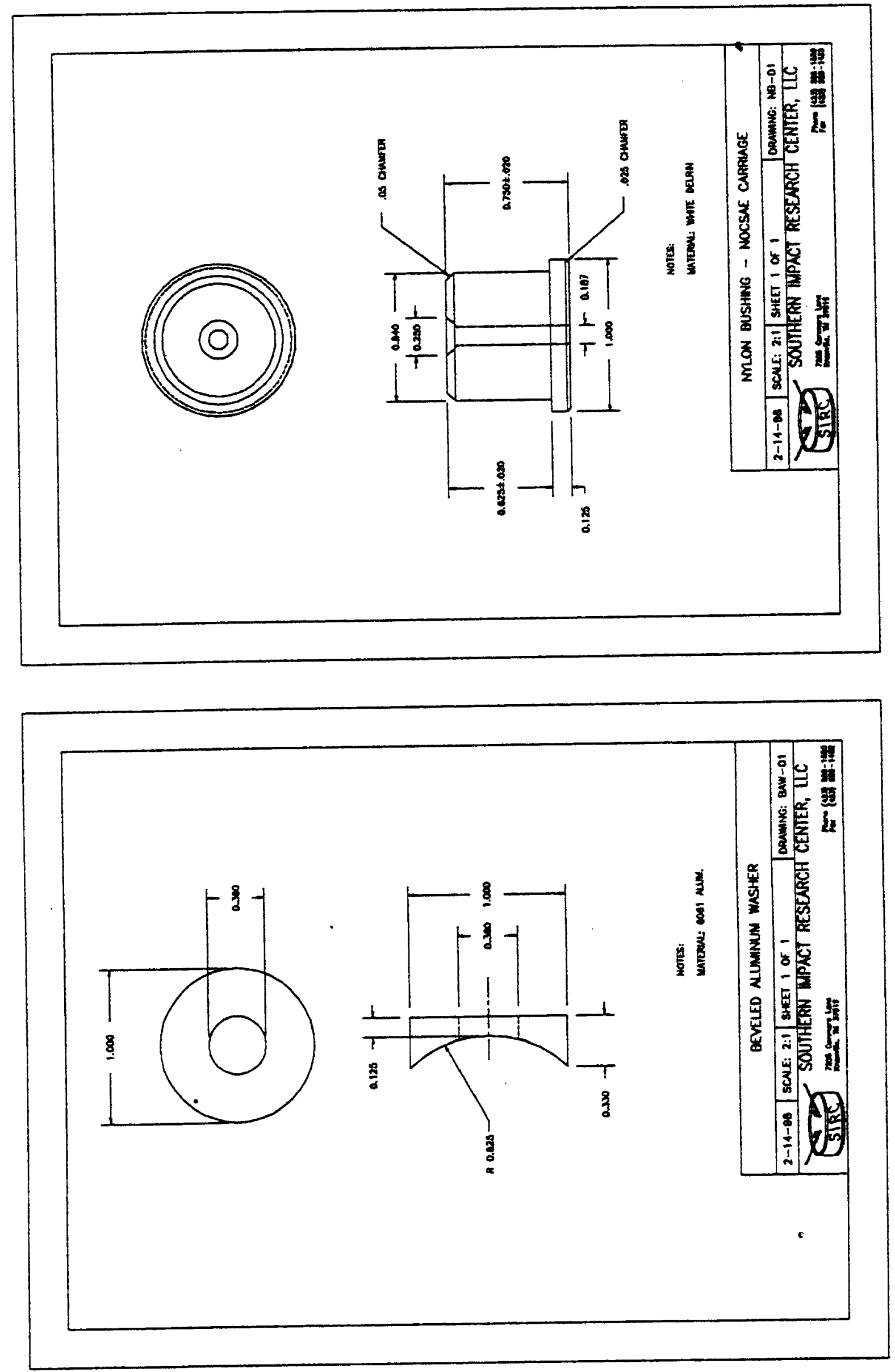

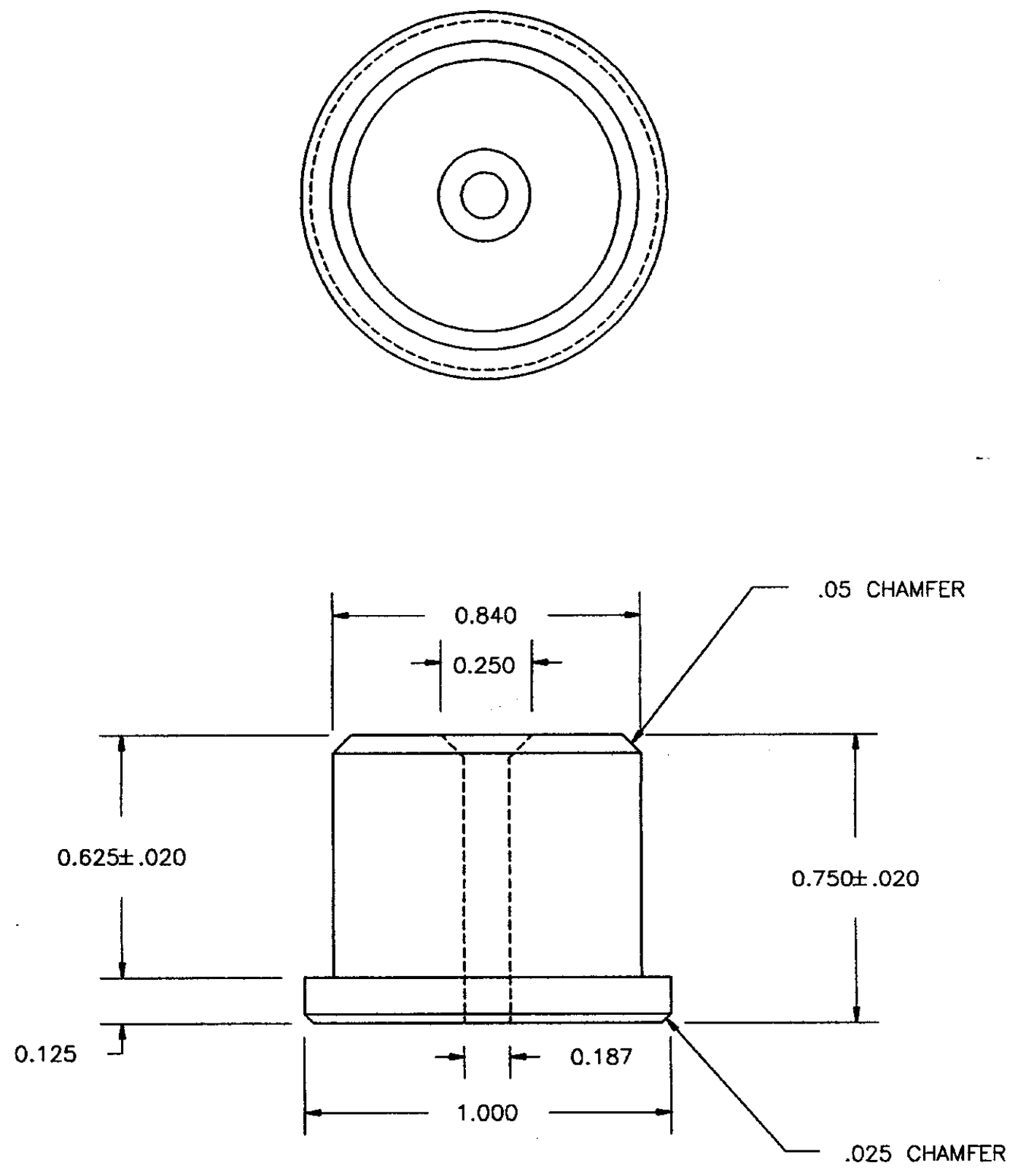

NOTES:

MATERIAL: WHITE DELRIN

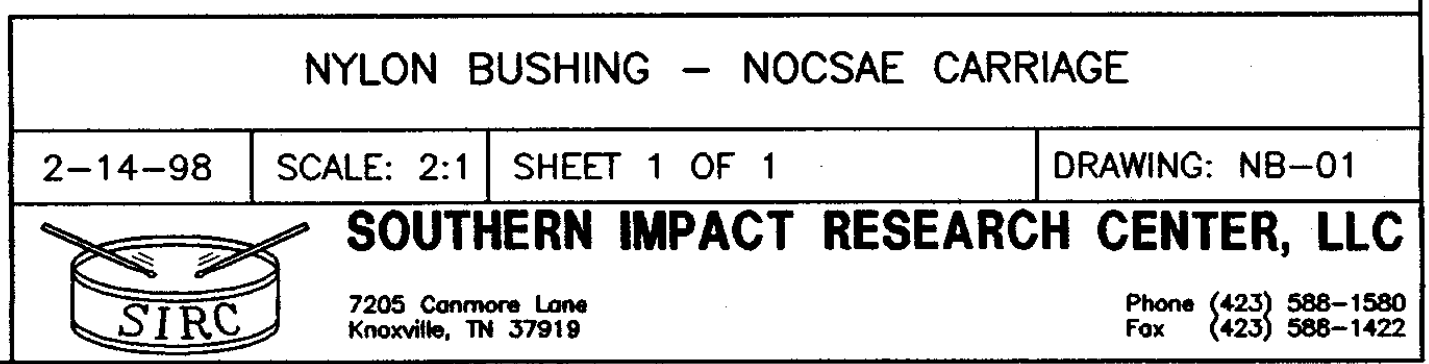




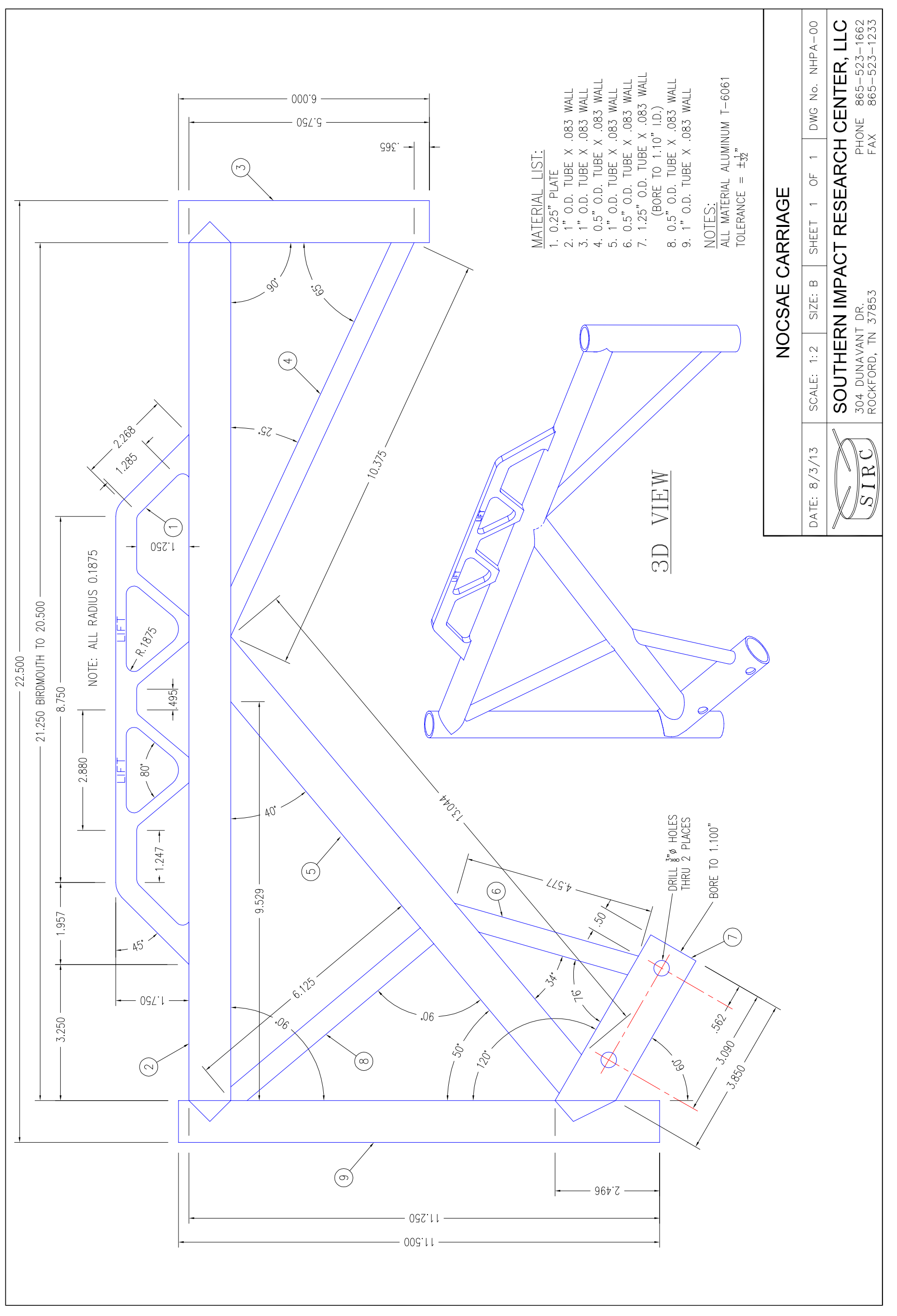




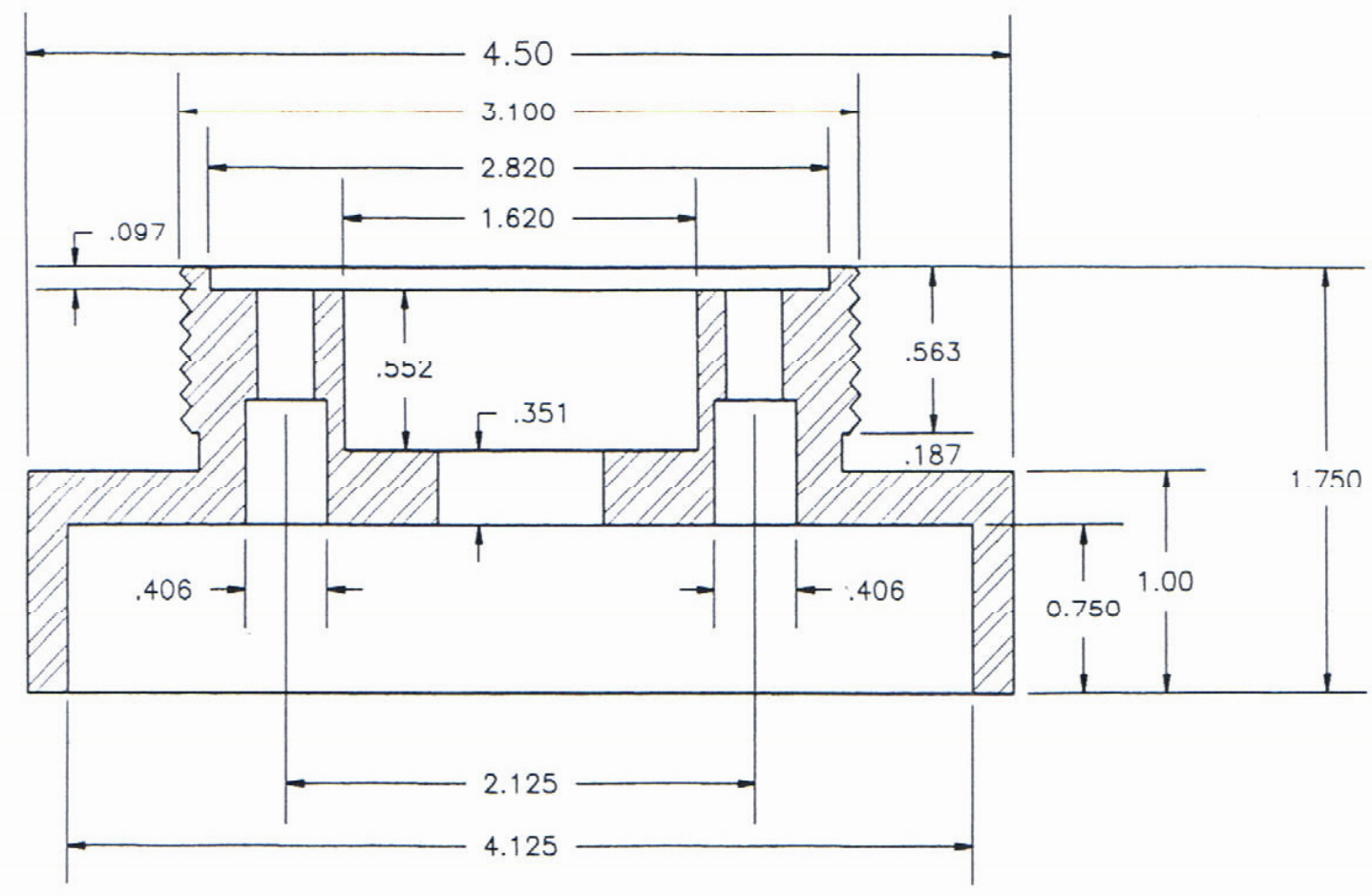

SECTION B-B

NOTES:

1. MATERIAL: 2024 T-351

2. PINS: $\quad 375$ DOWEL PIN $\times 3 / 4 "$ LONG

3. THD. SIZE: $31 / 8^{\prime \prime}-10 \mathrm{TPI}$

4. TOLERANCE: 3 PLACES \pm .0052 PLACES \pm .010

5. FINISH: $\checkmark 125$

6. DEBURR: BREAK ALL SHARP EDGES

\section{HEADFORM COLLAR}

\begin{tabular}{l|l} 
DATE: $11 / 1 / 97$ & SCALE: $1: 1$
\end{tabular}

SOUTHERN IMPACT RESEARCH CENTER, LLC
SIRC
Knoxville, TN 37919




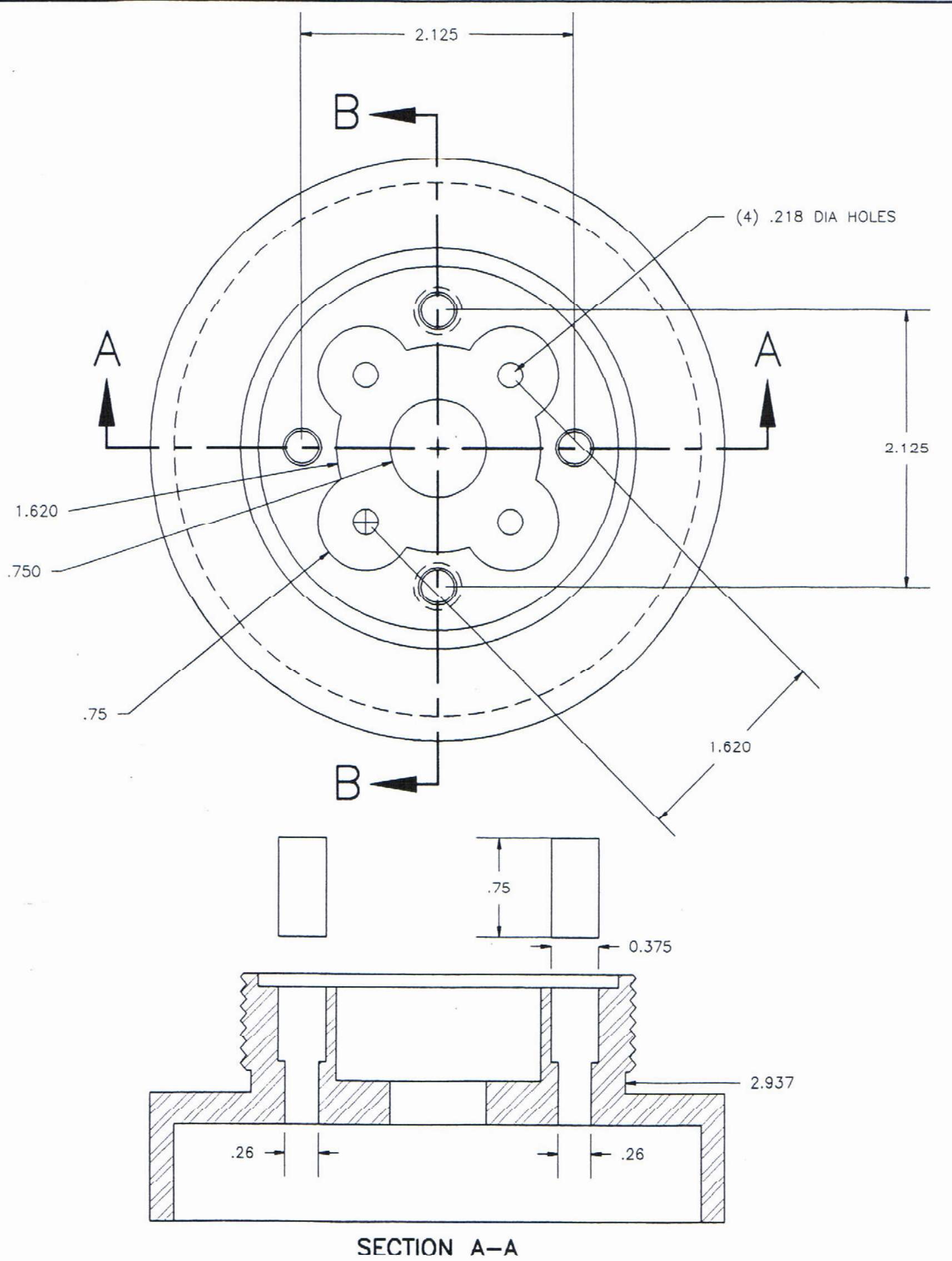



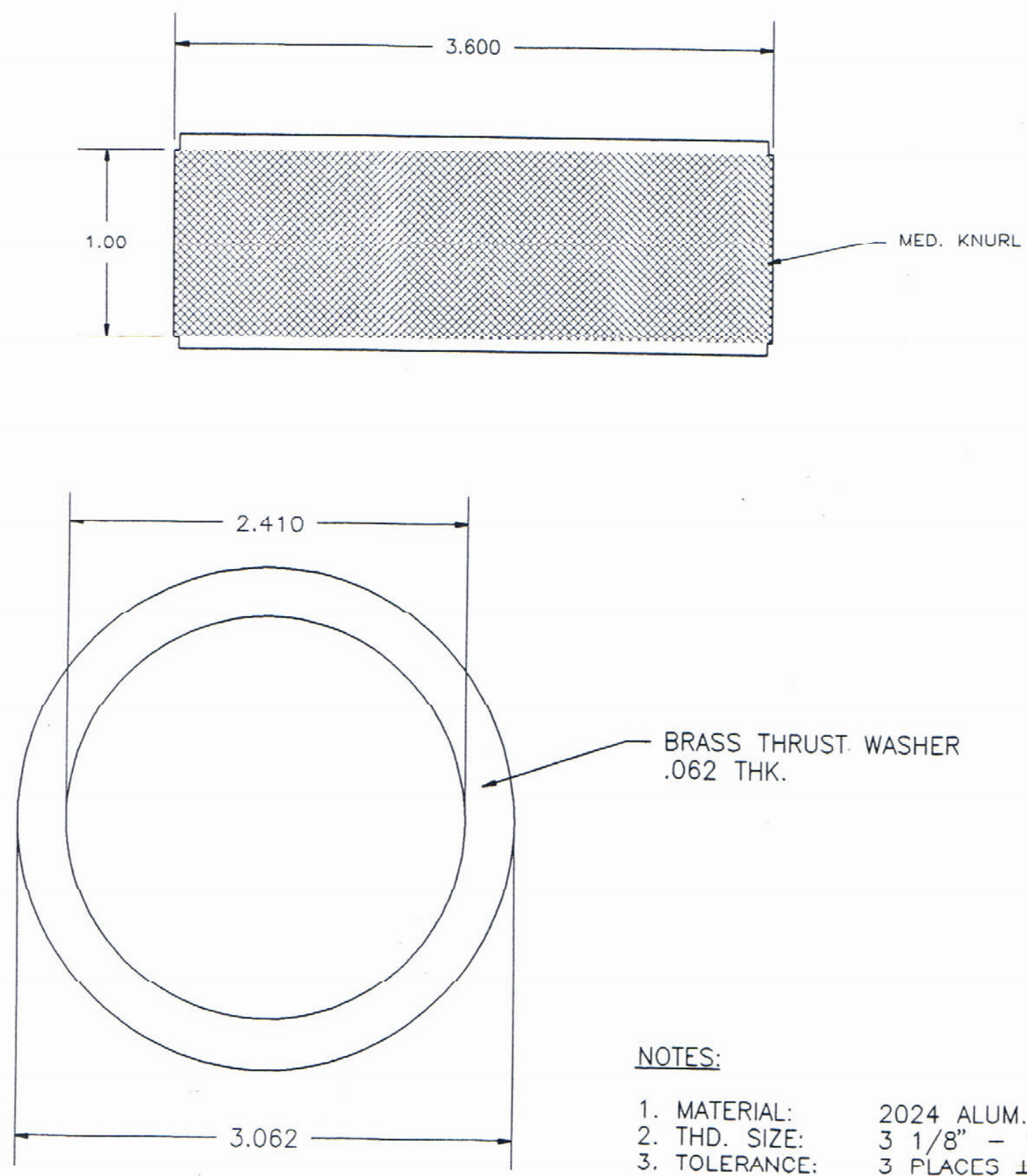

BRASS THRUST. WASHER .062 THK.

\section{NOTES:}

1. MATERIAL: 2024 ALUM.

2. THD. SIZE: $31 / 8^{\prime \prime}-10$

3. TOLERANCE: 3 PLACES $\perp .005$

4. FINISH: $\quad 125$

5. DEBURR: BREAK ALL SHARP EDGES

\section{HEADFORM THREADED LOCK RING}

DATE: $11 / 2 / 97 \quad$ SCALE: $1: 1$

SOUTHERN IMPACT RESEARCH CENTER, LLC 


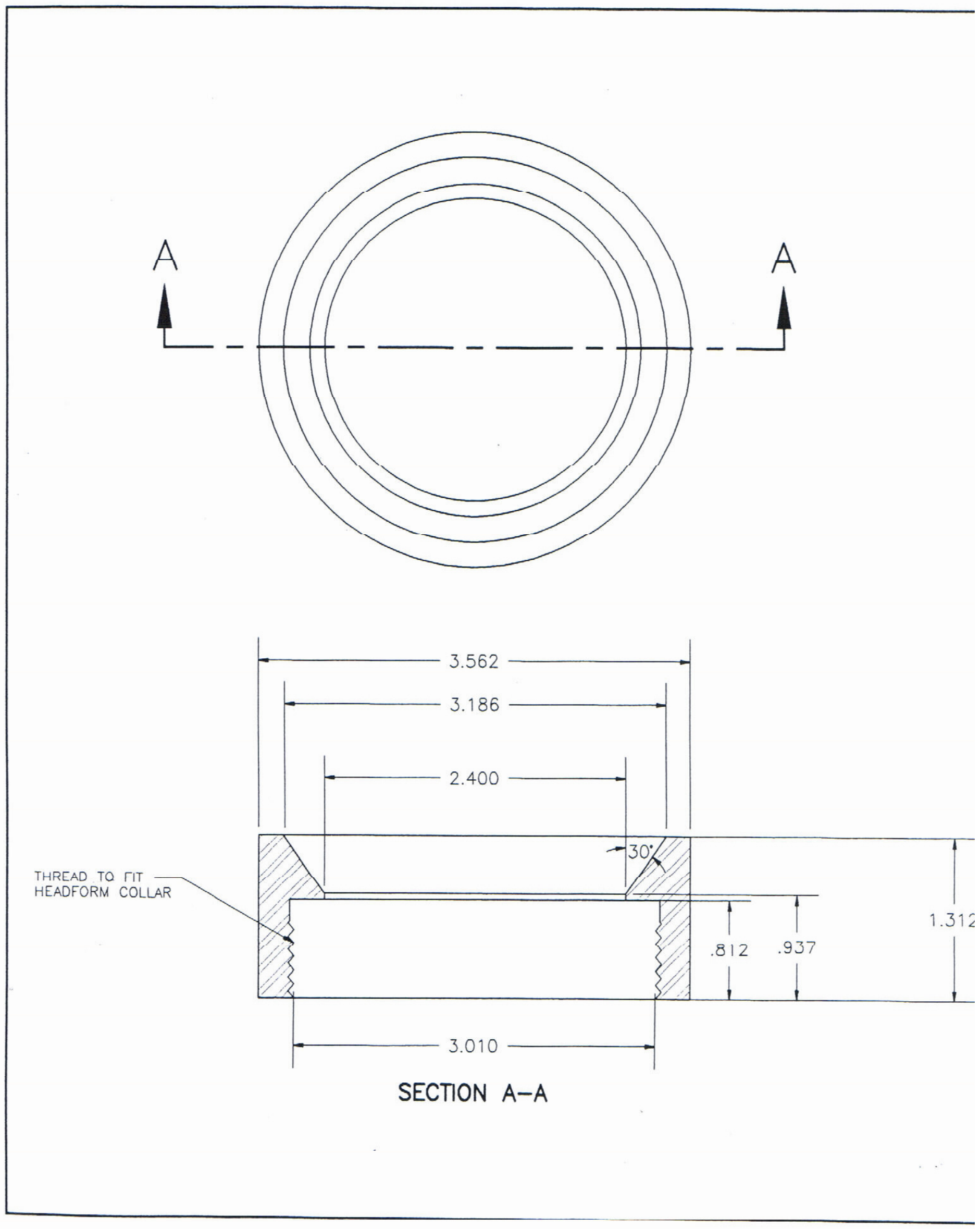




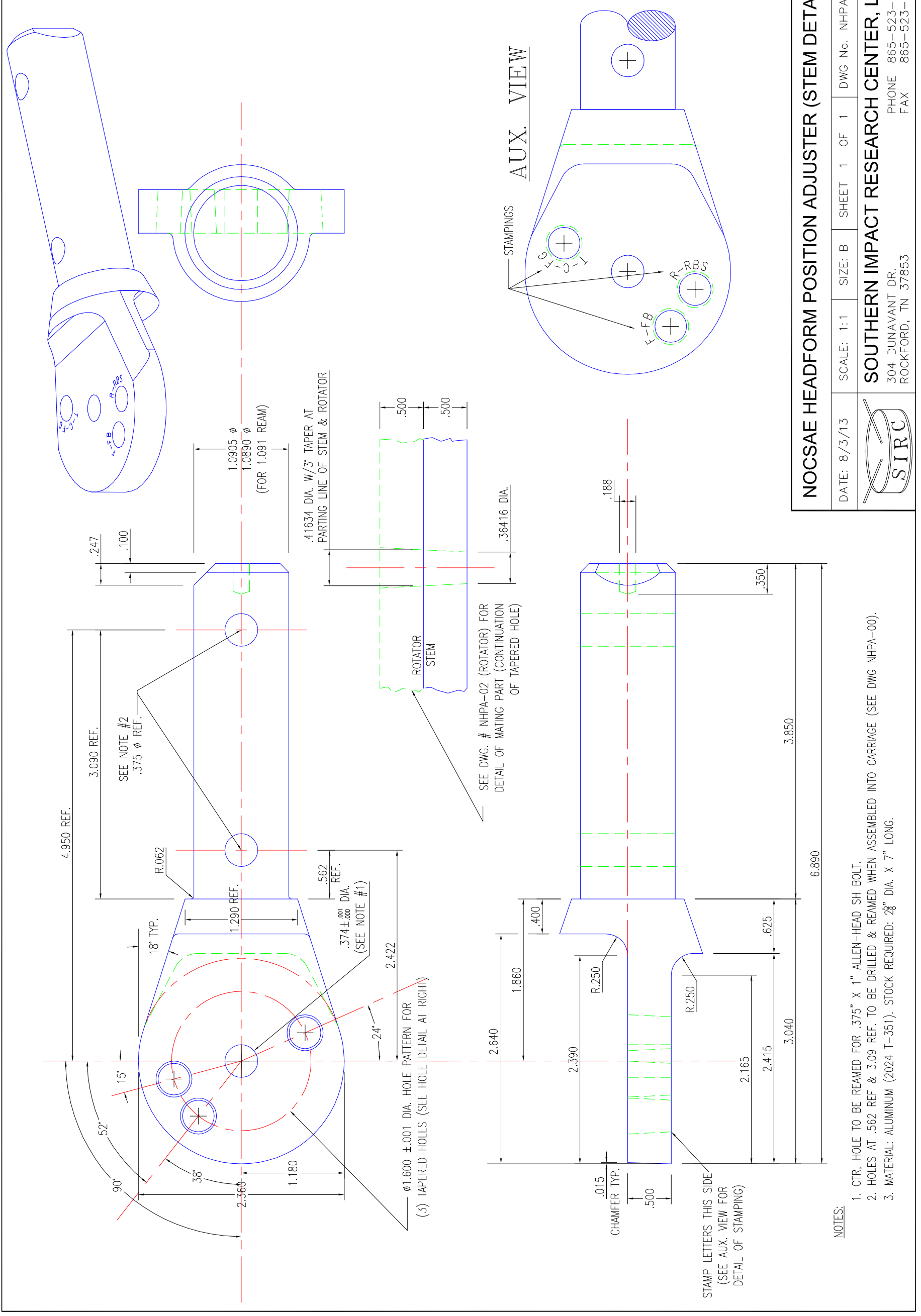




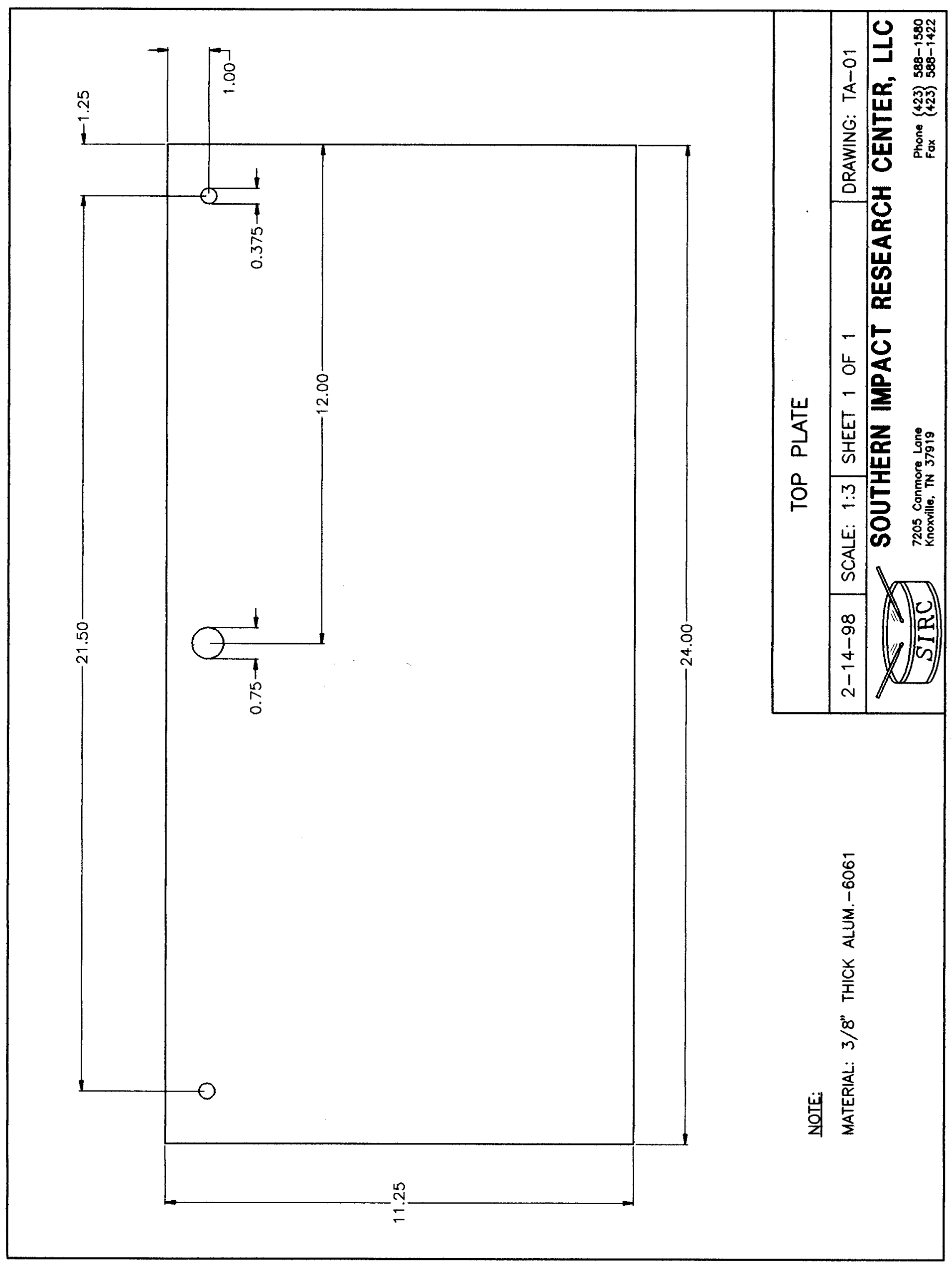


APPENDIX D: HELMET DROP TOWER ENGINEERING DRAWING 

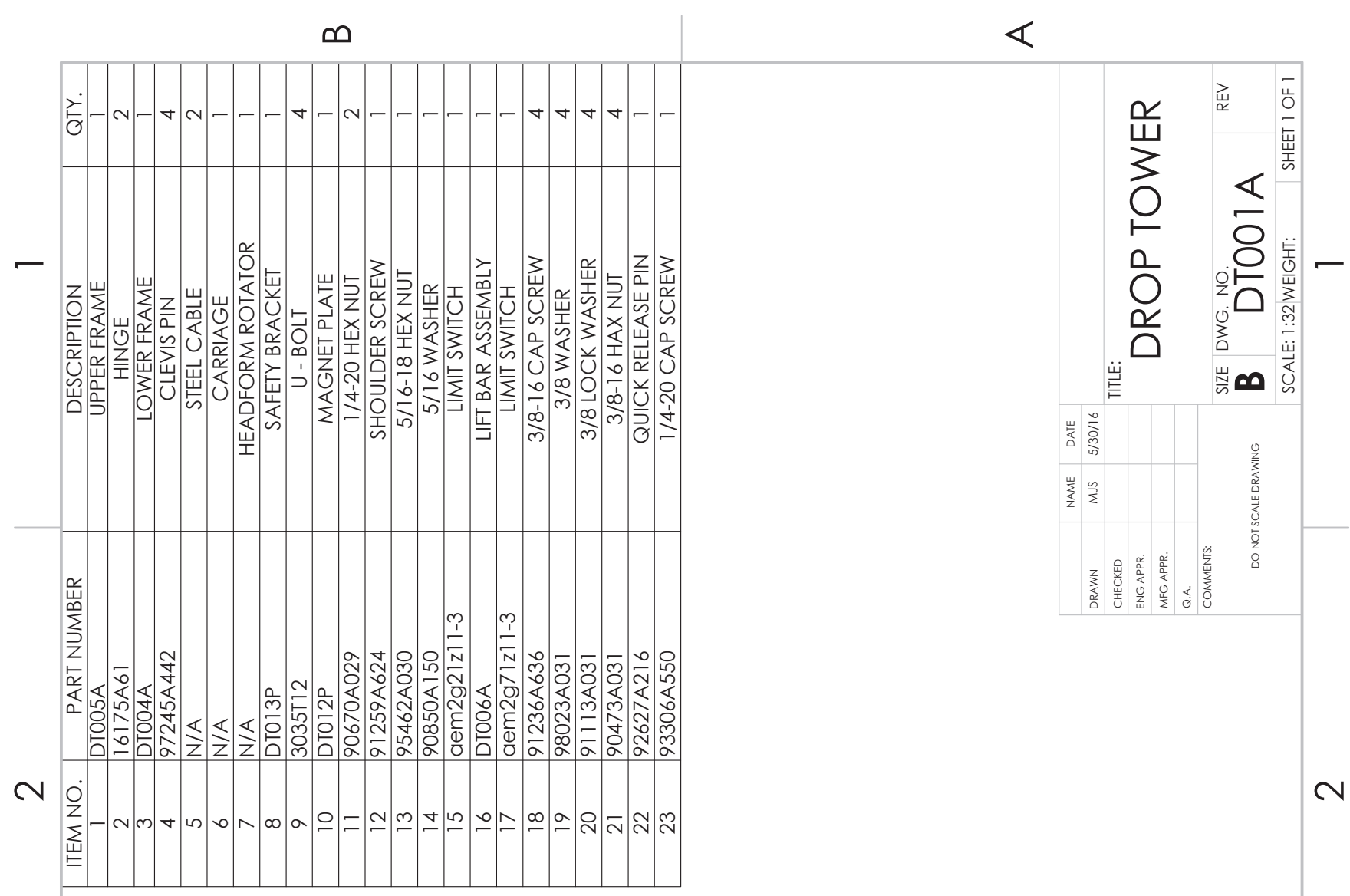

N

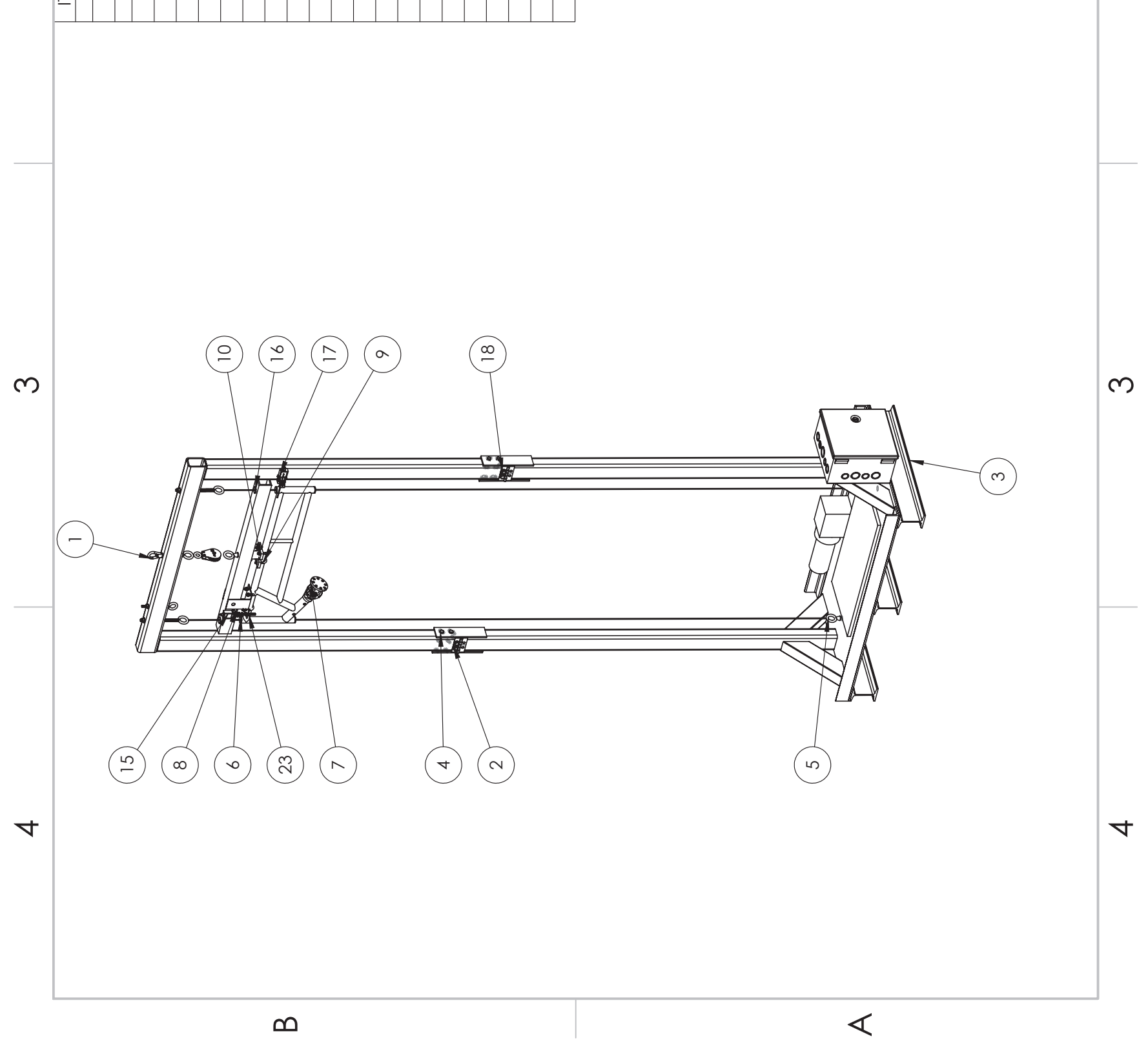




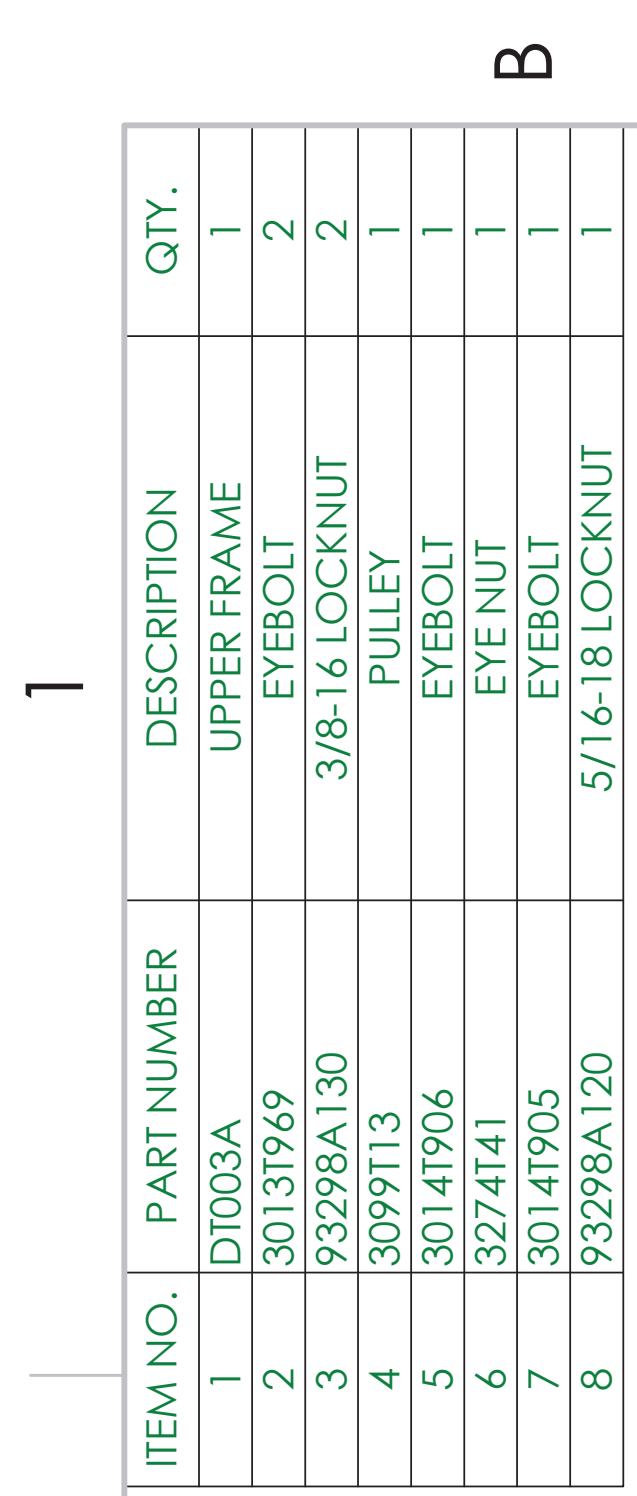

$\varangle$
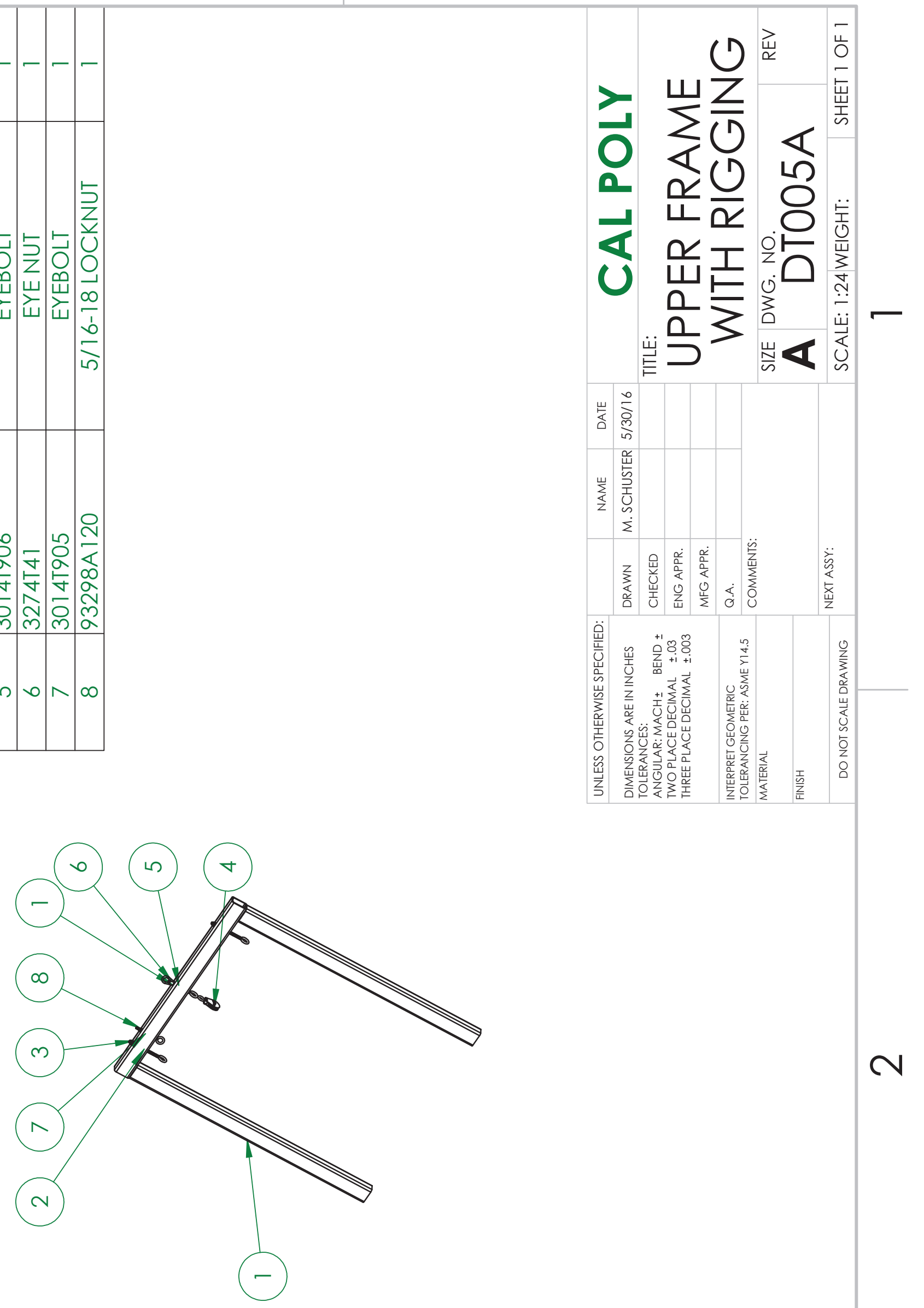


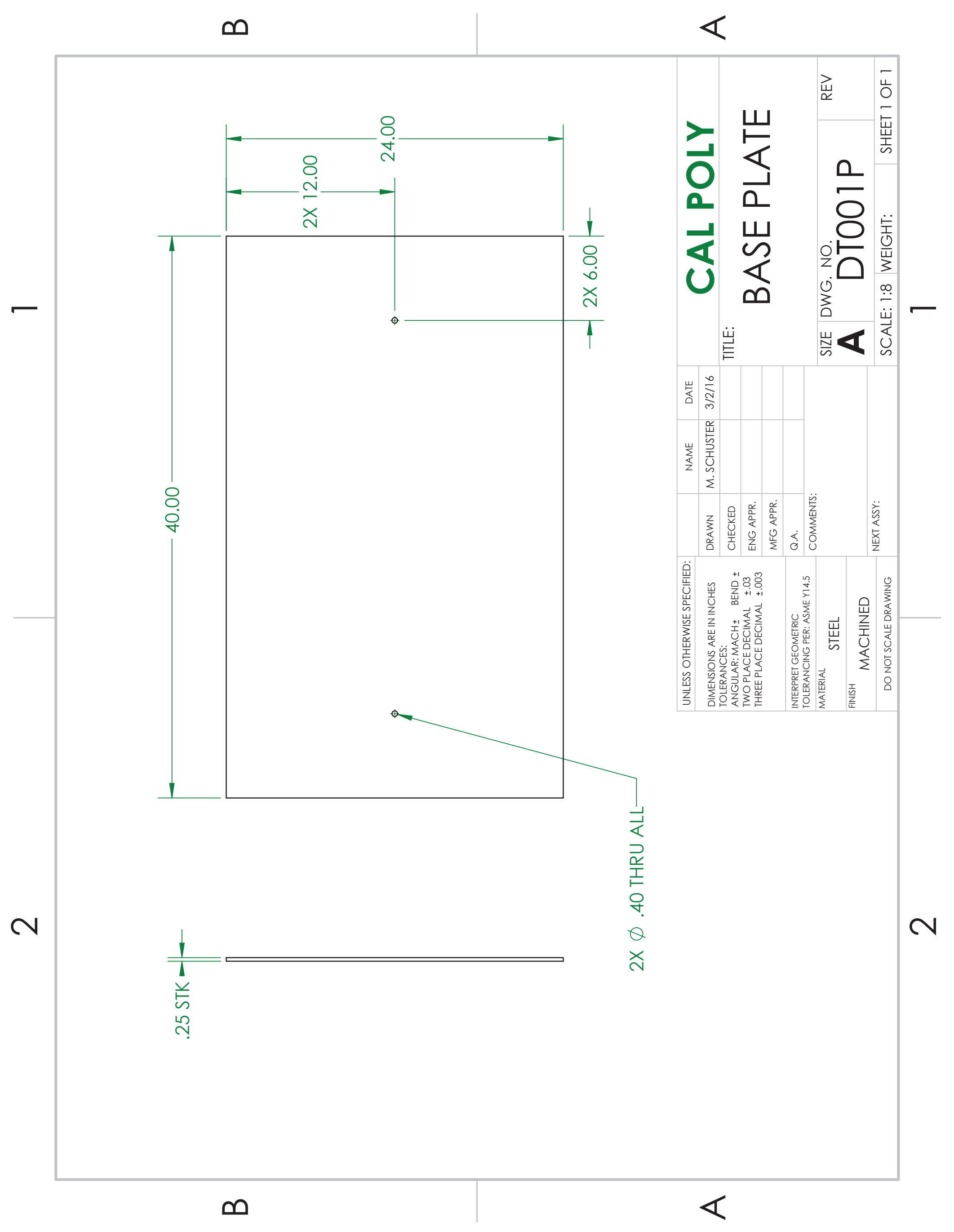



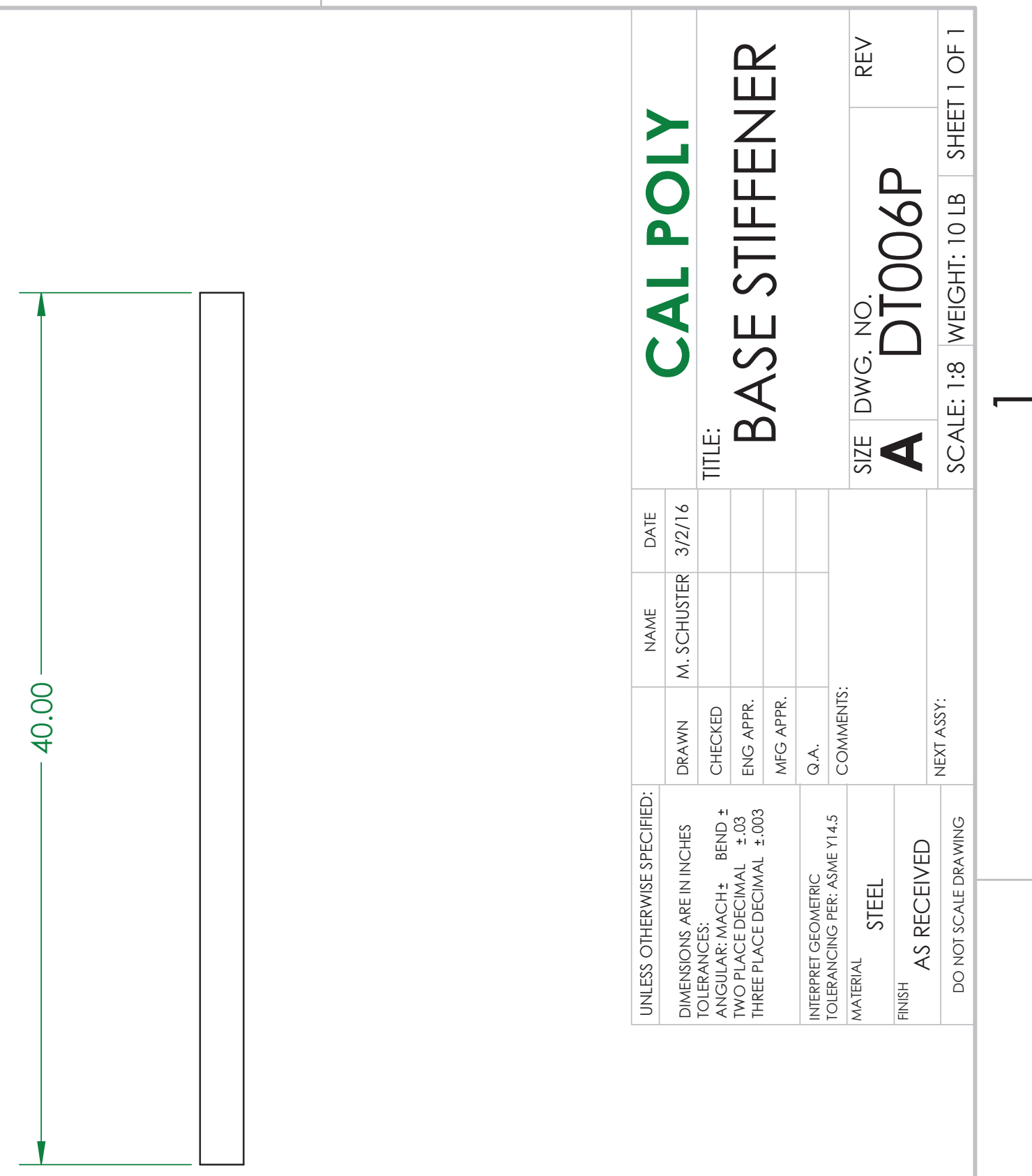

N

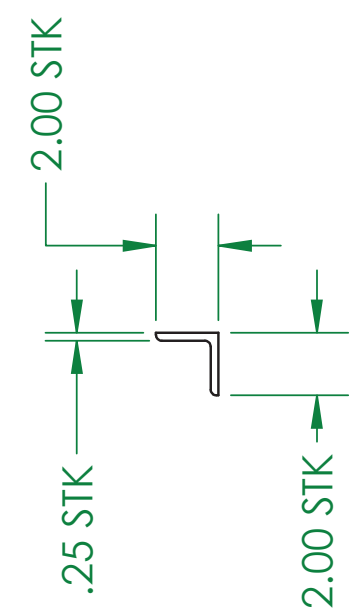




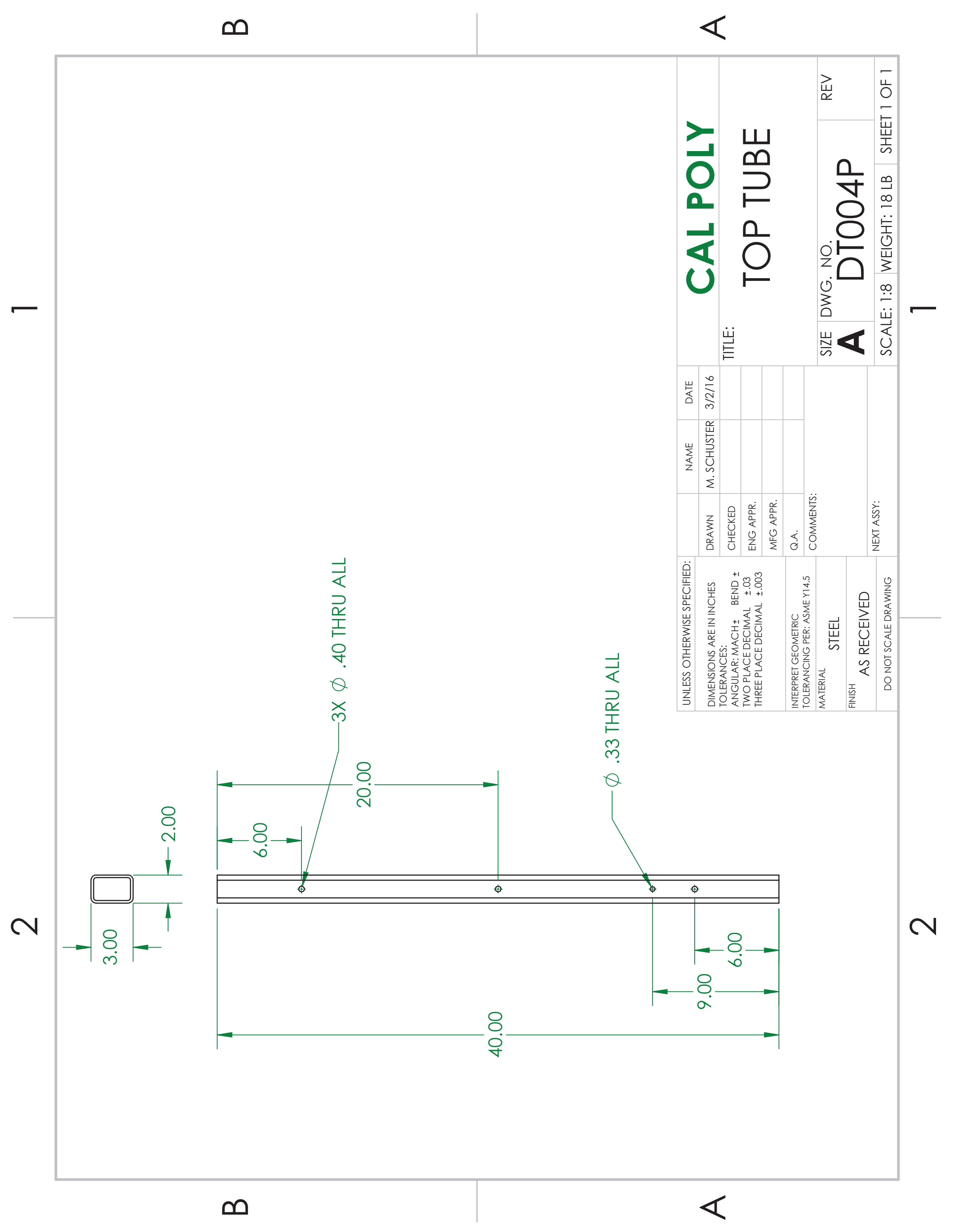



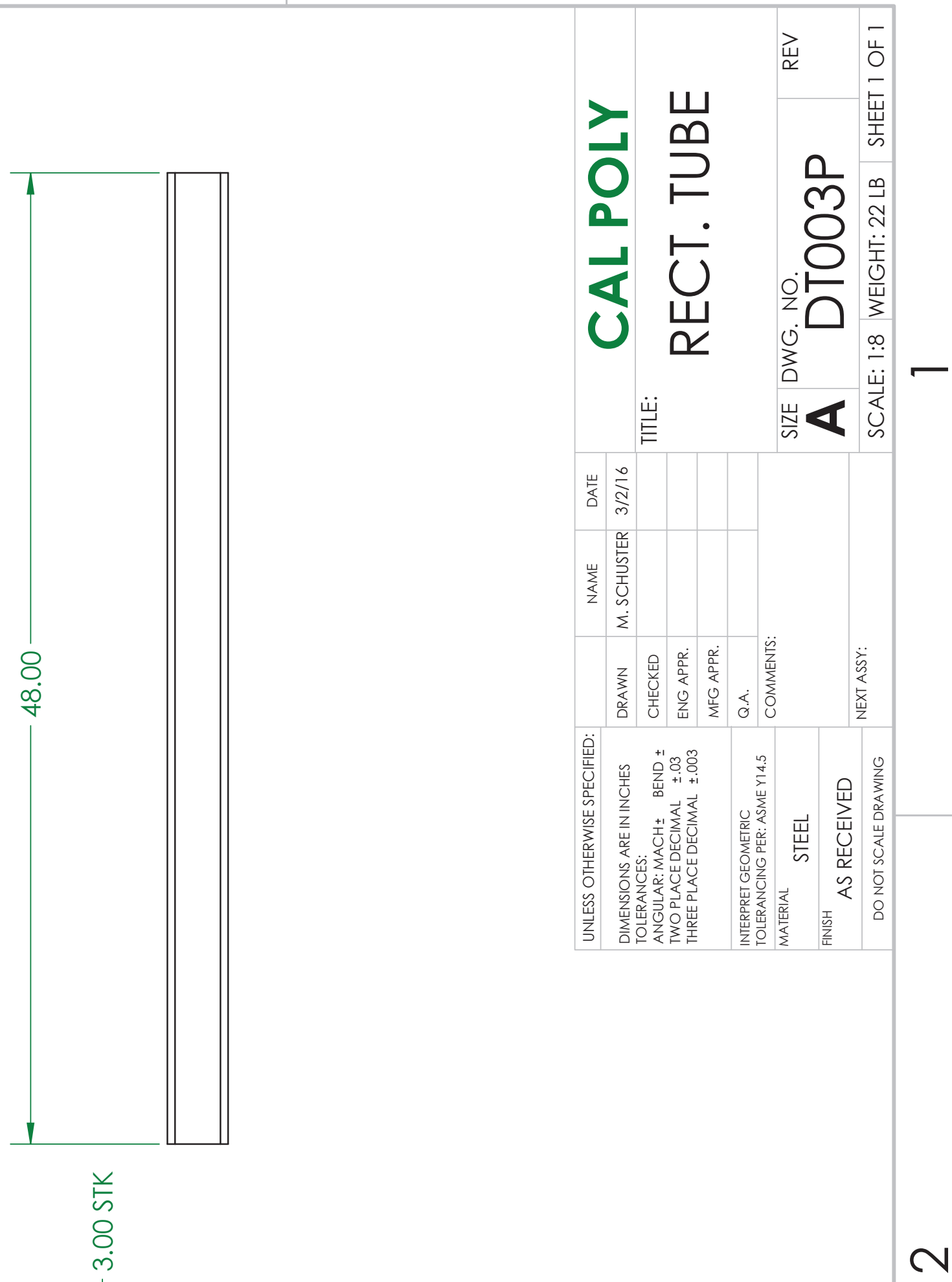

N

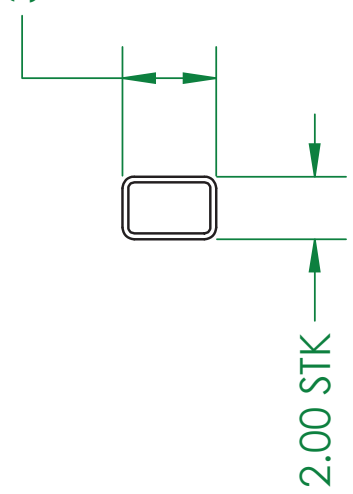



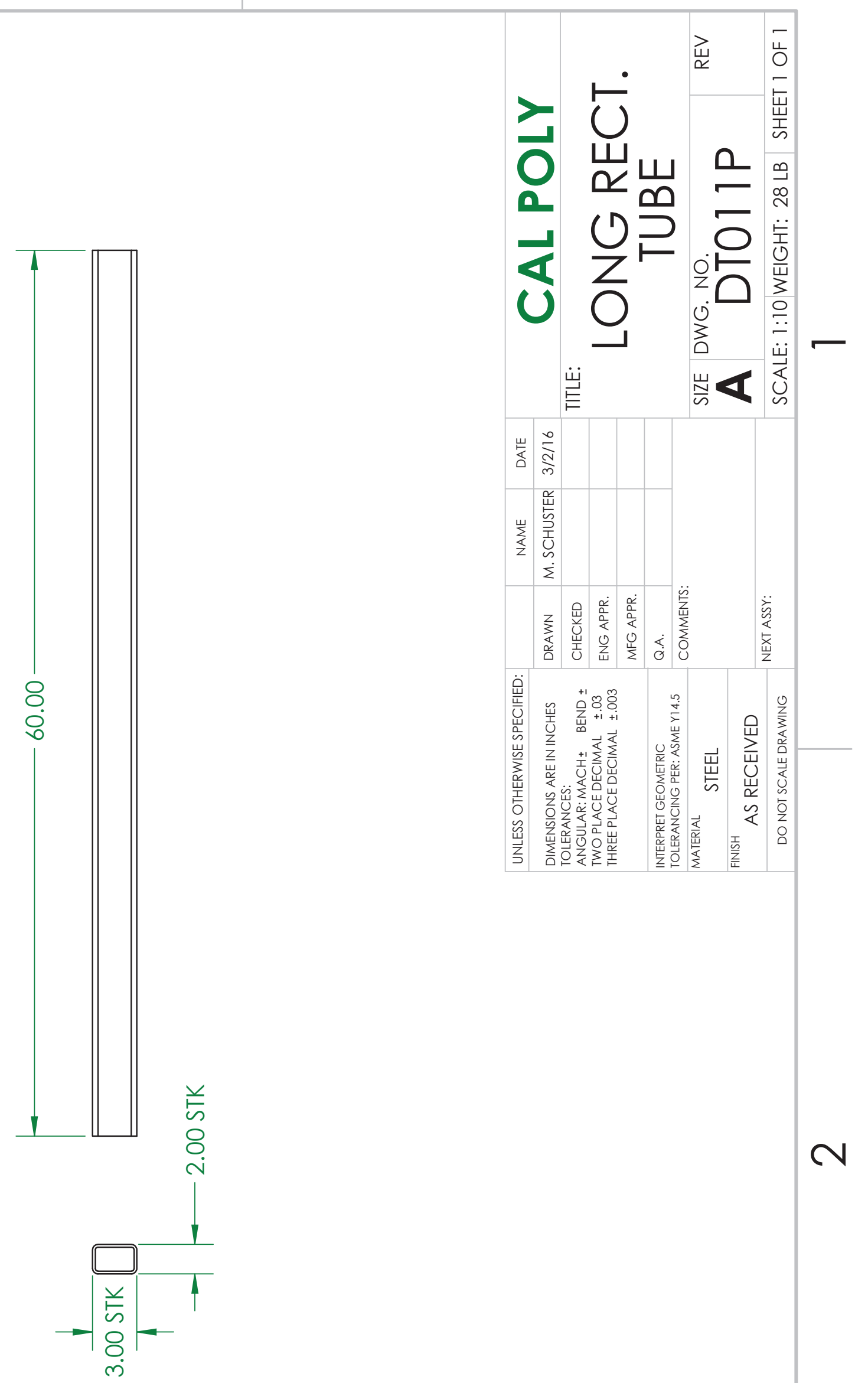


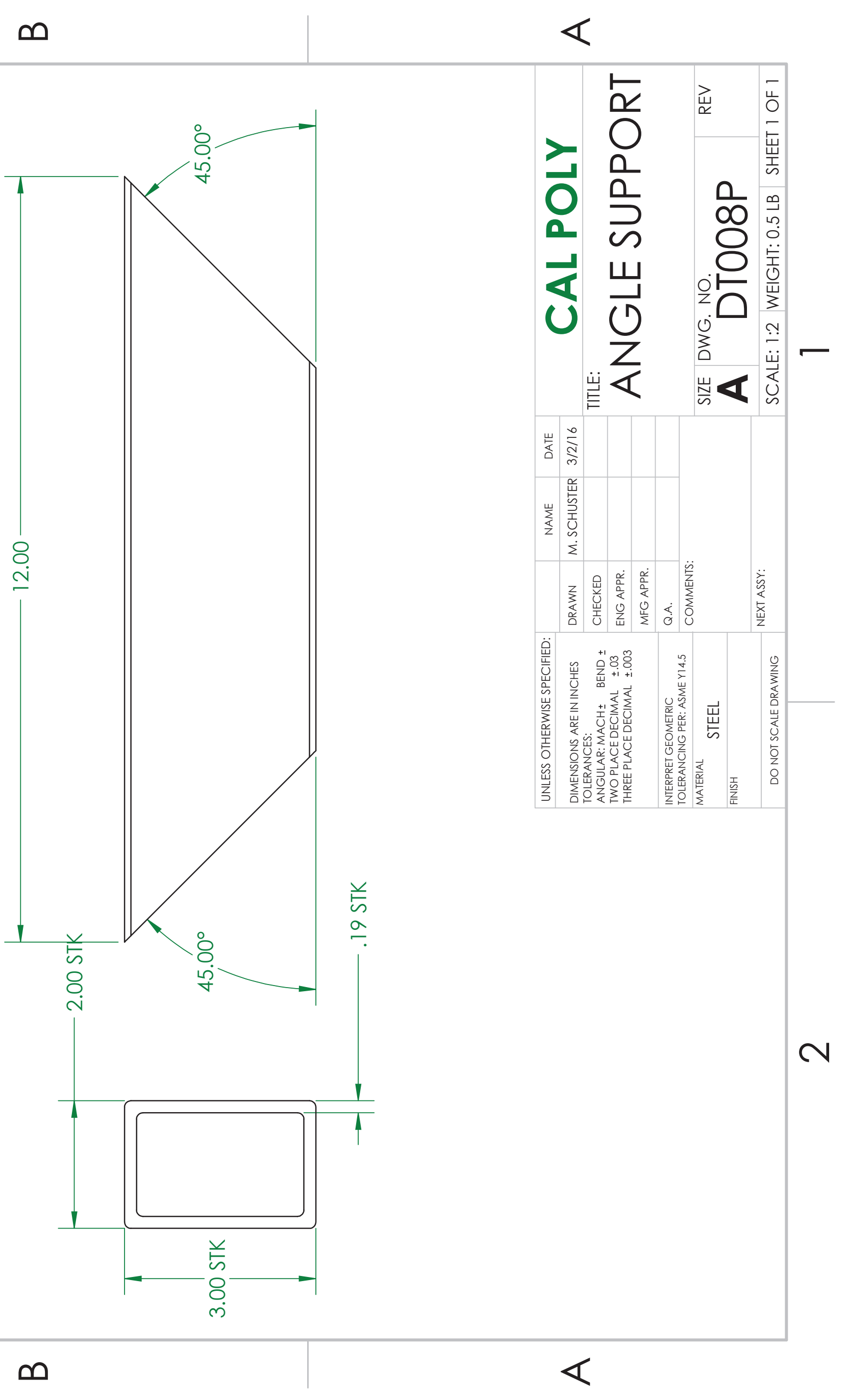




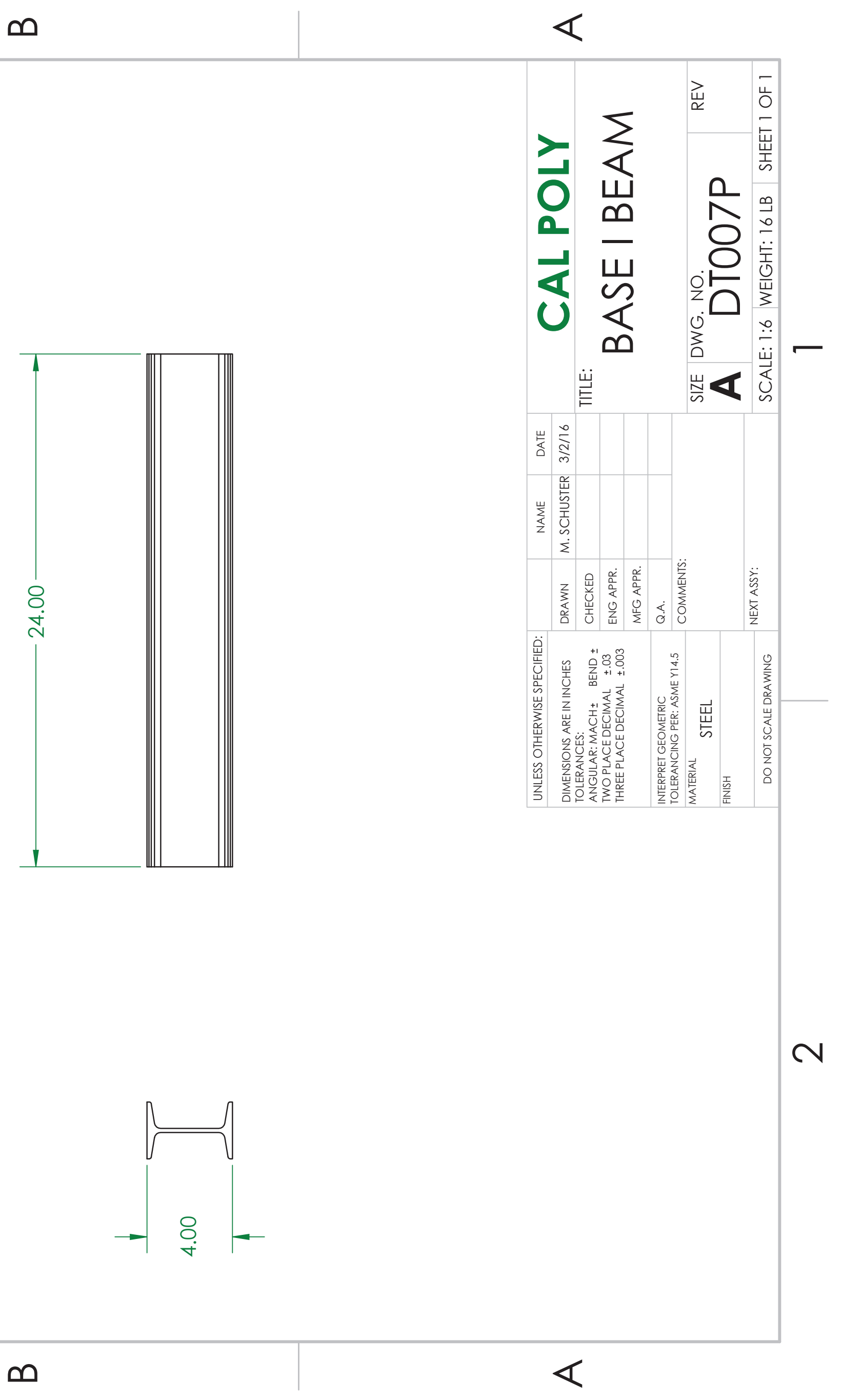



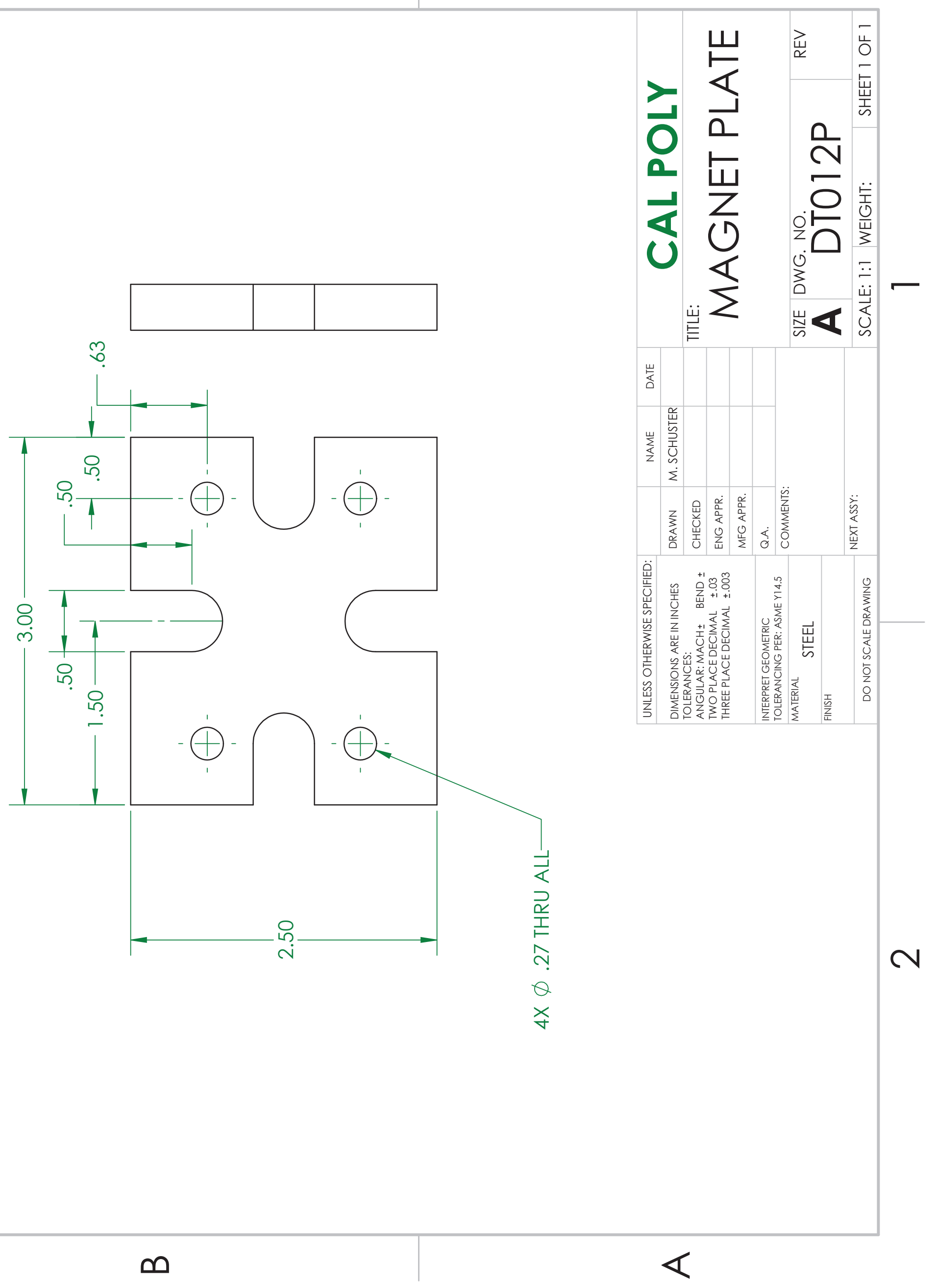

䋆
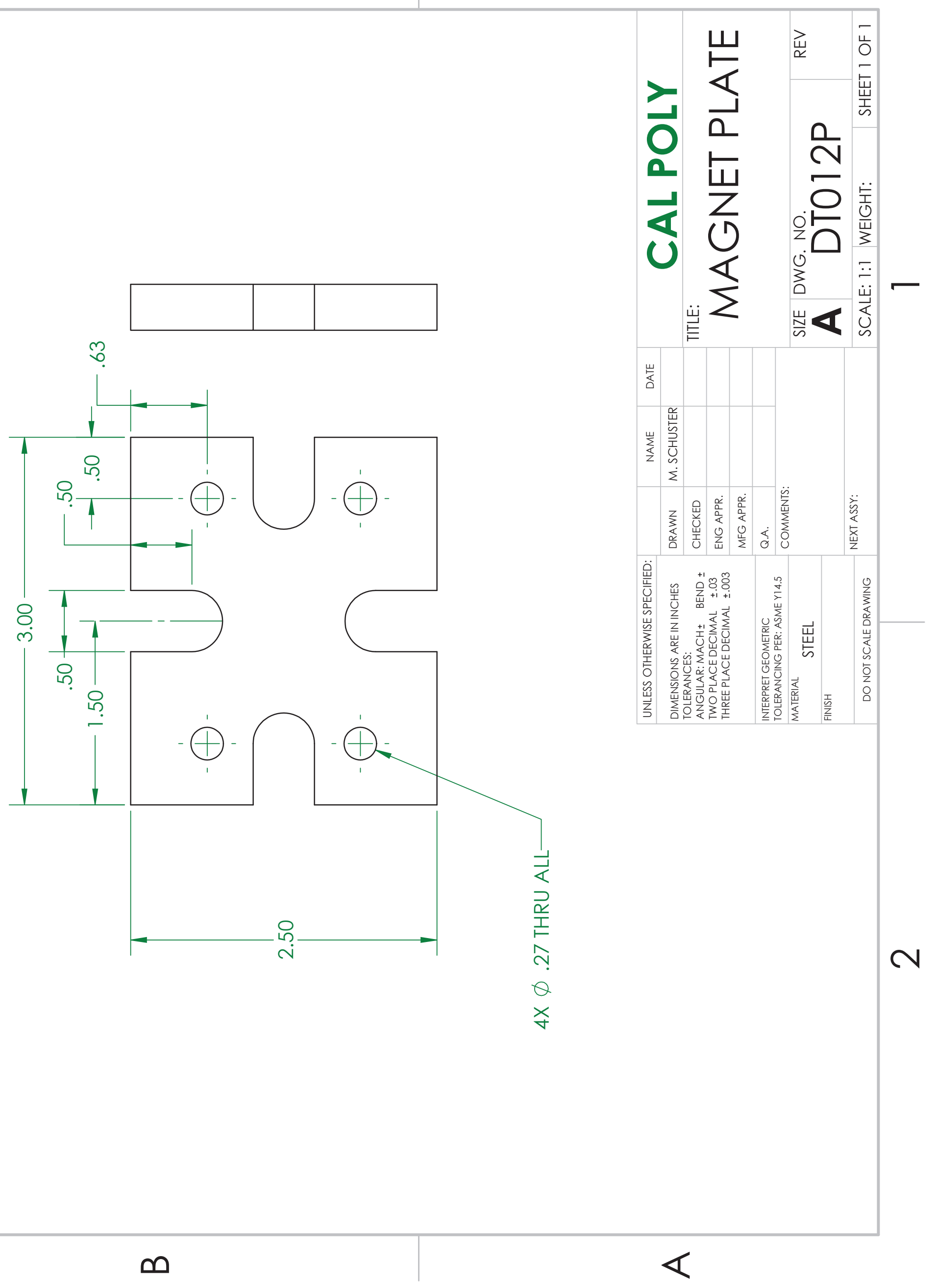

N 


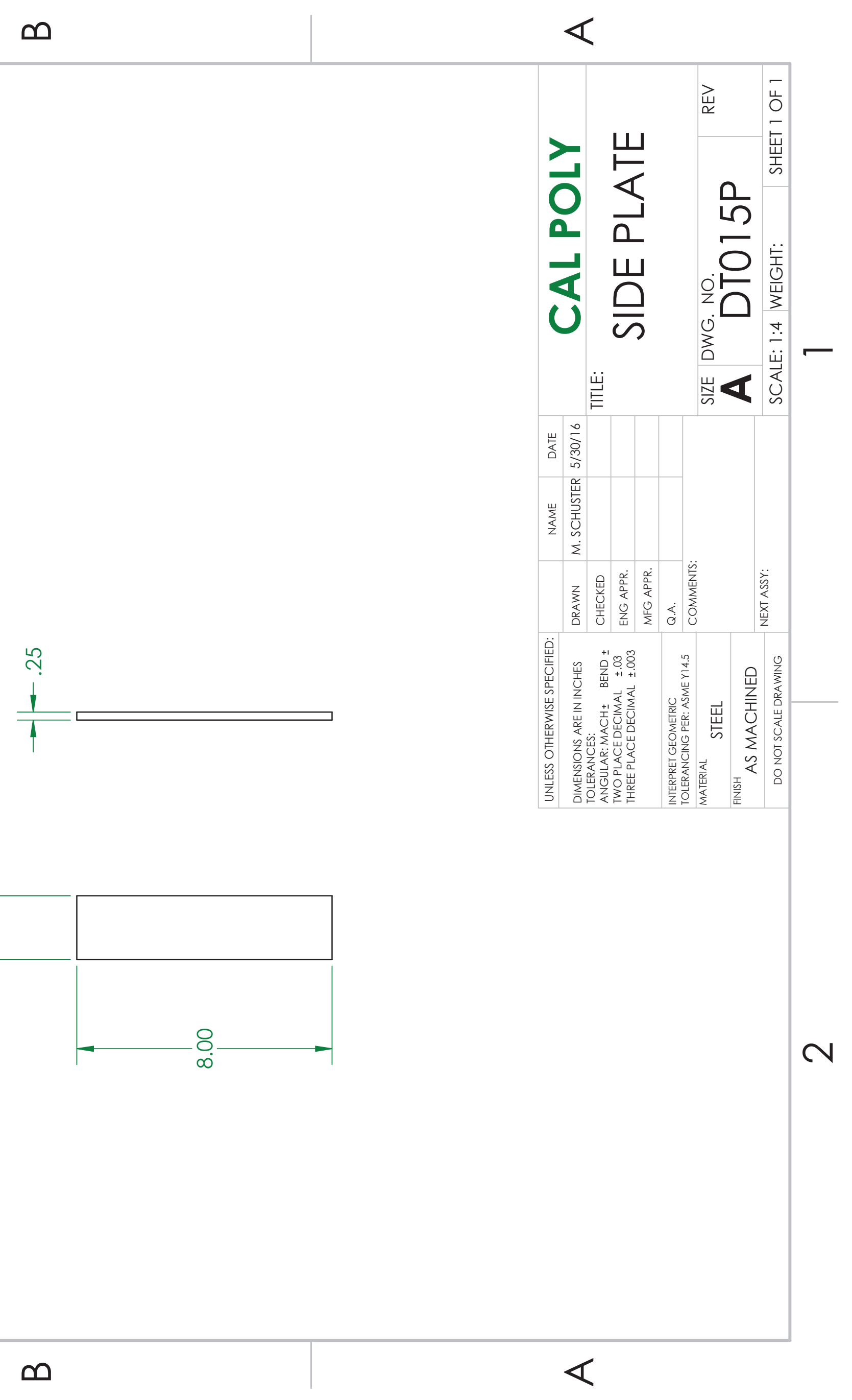




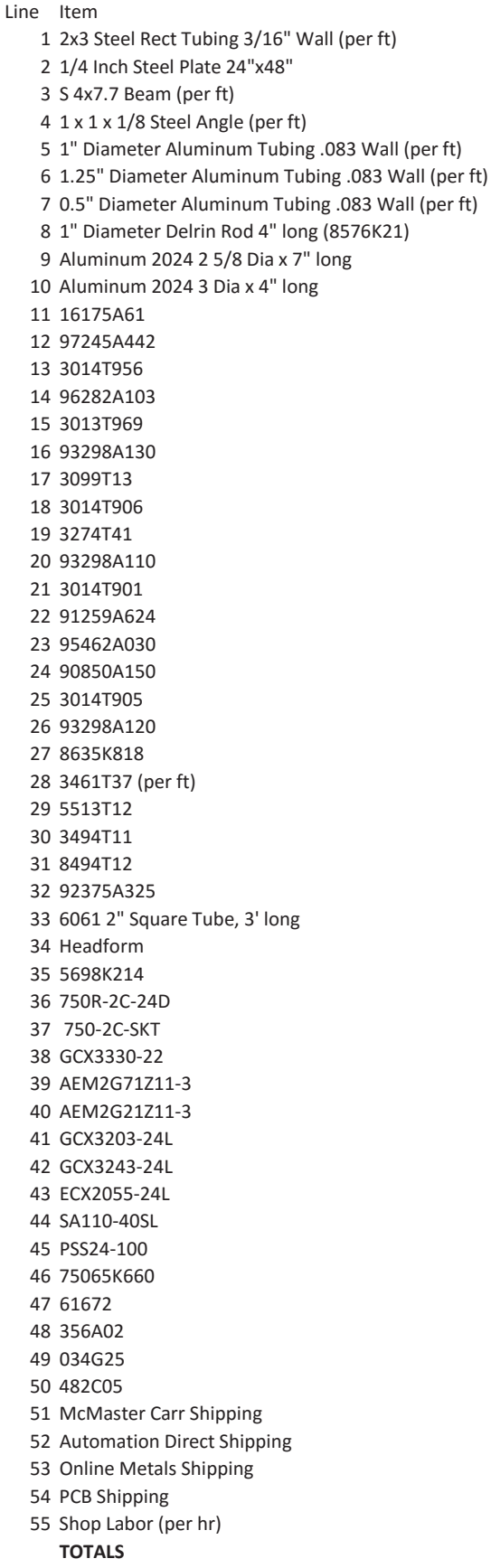

\begin{tabular}{|c|c|c|c|c|c|c|c|}
\hline \multicolumn{2}{|c|}{ Pkg QTY } & \multicolumn{2}{|c|}{ Net Price } & \multicolumn{2}{|c|}{ Price } & \multirow{2}{*}{$\begin{array}{l}\text { Source } \\
\text { B\&B Metals }\end{array}$} & \multirow{2}{*}{$\begin{array}{l}\text { Part } \\
\text { Frame }\end{array}$} \\
\hline 2 & & & & $\$$ & 104.00 & & \\
\hline 1 & & & & $\$$ & 70.00 & B\&B Metals & Frame \\
\hline 6 & & & & $\$$ & 50.00 & B\&B Metals & Frame \\
\hline 7 & & & & $\$$ & 15.00 & B\&B Metals & Frame \\
\hline 7 & & & & $\$$ & 32.52 & Online Metals & Carriage \\
\hline 1 & & & & $\$$ & 10.05 & Online Metals & Carriage \\
\hline 1 & & & & $\$$ & 5.72 & Online Metals & Carriage \\
\hline 1 & & & & $\$$ & 5.06 & McMaster & Bushings \\
\hline 1 & & & & $\$$ & 49.56 & Online Metals & Headform Stem \\
\hline 1 & & & & $\$$ & 40.40 & Online Metals & Headform Rotator \\
\hline 2 & 1 & $\$$ & 4.45 & $\$$ & 8.90 & McMaster & Surface-Mount Hinge \\
\hline 4 & 5 & $\$$ & 7.08 & $\$$ & 7.08 & McMaster & Clevis Pin, 1/2" Dia, 4" Len \\
\hline 2 & 1 & $\$$ & 3.15 & $\$$ & 6.30 & McMaster & Eyebolt with Shoulder, 3/8"-16 \\
\hline 2 & 50 & $\$$ & 6.98 & $\$$ & 6.98 & McMaster & Serrated-Flange Locknut, 3/8"-16 \\
\hline 2 & 1 & $\$$ & 8.23 & $\$$ & 16.46 & McMaster & Eyebolt without Shoulder, 3/8"-16 \\
\hline 2 & 50 & $\$$ & 7.60 & $\$$ & 7.60 & McMaster & Nylon-Insert Flange Locknut \\
\hline 1 & 1 & $\$$ & 11.88 & $\$$ & 11.88 & McMaster & Pulley \\
\hline 1 & 1 & $\$$ & 13.88 & $\$$ & 13.88 & McMaster & Eyebolt with Shoulder, 3/8"-16 \\
\hline 1 & 1 & $\$$ & 6.83 & $\$$ & 6.83 & McMaster & Oval Eye Nut \\
\hline 2 & 100 & $\$$ & 7.48 & $\$$ & 7.48 & McMaster & Nylon-Insert Nonmarring Flange Locknut \\
\hline 2 & 1 & $\$$ & 10.22 & $\$$ & 20.44 & McMaster & Eyebolt with Shoulder, 1/4"-20 Thread Size \\
\hline 1 & 1 & $\$$ & 1.54 & $\$$ & 1.54 & McMaster & Shoulder Screw, 3/8" Dia, 5/16"-18 \\
\hline 1 & 100 & $\$$ & 6.44 & $\$$ & 6.44 & McMaster & Hex Nut, 5/16"-18 \\
\hline 1 & 50 & $\$$ & 8.55 & $\$$ & 8.55 & McMaster & Flat Washer, 5/16" Screw Size \\
\hline 1 & 1 & $\$$ & 13.94 & $\$$ & 13.94 & McMaster & Eyebolt with Shoulder, 5/16"-18 \\
\hline 1 & 50 & $\$$ & 5.45 & $\$$ & 5.45 & McMaster & Flange Locknut, 5/16"-18 \\
\hline 1 & 1 & $\$$ & 53.45 & $\$$ & 53.45 & McMaster & Rubber, $1 / 2$ " Thick, 12 " x 24", 40A Duro \\
\hline 5 & 1 & $\$$ & 0.87 & $\$$ & 21.75 & McMaster & Stainless Steel Wire Rope, 1/8" Dia \\
\hline 4 & 1 & $\$$ & 3.53 & $\$$ & 14.12 & McMaster & Wire Rope Clamp \\
\hline 4 & 1 & $\$$ & 0.63 & $\$$ & 2.52 & McMaster & Wire Rope Thimble \\
\hline 1 & 1 & $\$$ & 5.36 & $\$$ & 5.36 & McMaster & Anchor Shackle \\
\hline 4 & 10 & $\$$ & 10.23 & $\$$ & 10.23 & McMaster & Hairpin Cotter Pin \\
\hline 1 & & $\$$ & 27.83 & $\$$ & 27.83 & Online Metals & Lift Bar \\
\hline 1 & & $\$$ & 500.00 & $\$$ & 500.00 & & \\
\hline 1 & & $\$$ & 49.48 & $\$$ & 49.48 & McMaster & 24V Electromagnet \\
\hline 4 & & $\$$ & 7.75 & $\$$ & 31.00 & Automation Direct & 24VDC Coil DPDT Relay \\
\hline 4 & & $\$$ & 4.25 & $\$$ & 17.00 & Automation Direct & Relay Socket \\
\hline 1 & & $\$$ & 14.50 & $\$$ & 14.50 & Automation Direct & 3 Pos Knob, Return to Center \\
\hline 1 & & $\$$ & 28.00 & $\$$ & 28.00 & Automation Direct & Rod Limit Switch \\
\hline 1 & & $\$$ & 26.00 & $\$$ & 26.00 & Automation Direct & Plunger Limit Switch \\
\hline 1 & & $\$$ & 14.00 & $\$$ & 14.00 & Automation Direct & Pushbutton \\
\hline 1 & & $\$$ & 15.50 & $\$$ & 15.50 & Automation Direct & 2 Pos Knob, Mom \\
\hline 1 & & $\$$ & 8.50 & $\$$ & 8.50 & Automation Direct & LED Indicator \\
\hline 1 & & $\$$ & 13.50 & $\$$ & 13.50 & Automation Direct & 4 Hole Pushbutton Enclosure \\
\hline 1 & & $\$$ & 30.00 & $\$$ & 30.00 & Automation Direct & 24V Power Supply \\
\hline 1 & & $\$$ & 36.21 & $\$$ & 36.21 & McMaster & Enclosure (Relays and P.S.) \\
\hline & & $\$$ & 149.99 & $\$$ & 149.99 & Harbor Freight & Winch \\
\hline 1 & & $\$$ & 895.50 & $\$$ & 895.50 & PCB PiezoTronics & Triaxial Accel \\
\hline 1 & & $\$$ & 211.50 & $\$$ & 211.50 & PCB PiezoTronics & 25' Accel Cable \\
\hline 1 & & $\$$ & 481.50 & $\$$ & 481.50 & PCB PiezoTronics & 4-Channel Accel Power Supply \\
\hline & & & & $\$$ & 50.00 & & \\
\hline & & & & $\$$ & - & & \\
\hline & & & & $\$$ & 24.10 & & \\
\hline & & & & $\$$ & - & & \\
\hline & & $\$$ & 16.00 & $\$$ & 320.00 & & \\
\hline & & & & $\$$ & $3,583.60$ & & \\
\hline
\end{tabular}


APPENDIX E: FULL SCALE TESTING ANALYSIS CODE 


\section{Table of Contents}

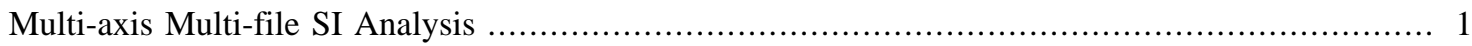

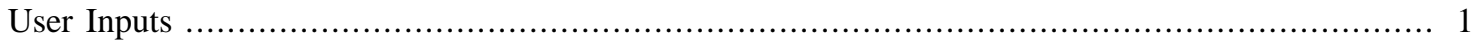

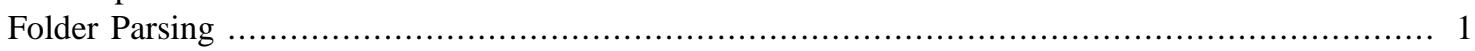

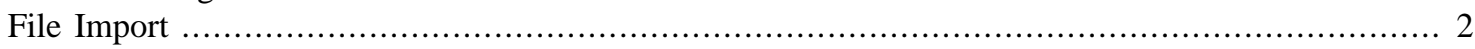

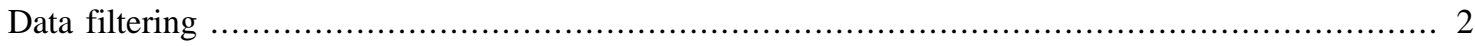

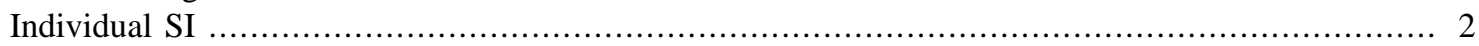

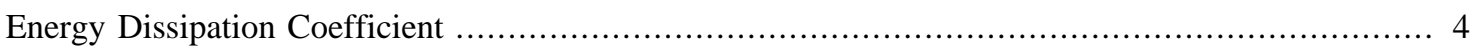

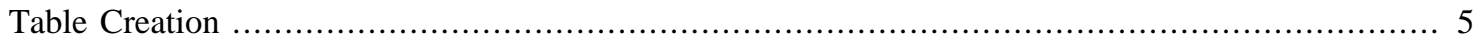

\section{Multi-axis Multi-file SI Analysis}

Michael Schuster 5/24/2016

analyses data to determine SI

clear all

clc

fprintf('Multi-axis Multi-file SI Analyzer $\backslash n$ ')

fprintf('Michael Schuster $\backslash n \backslash n$ ')

\section{User Inputs}

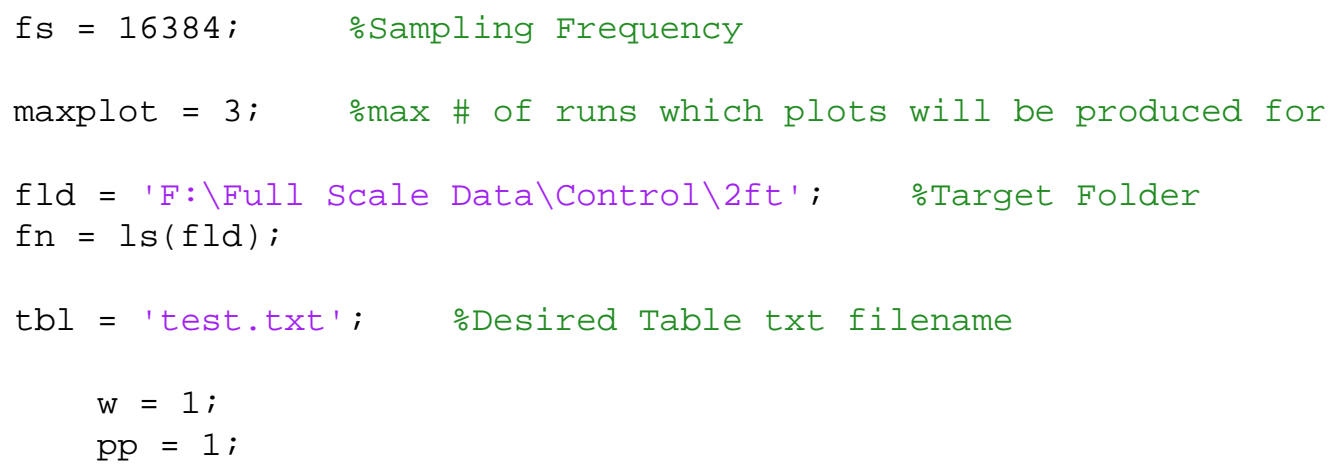

\section{Folder Parsing}

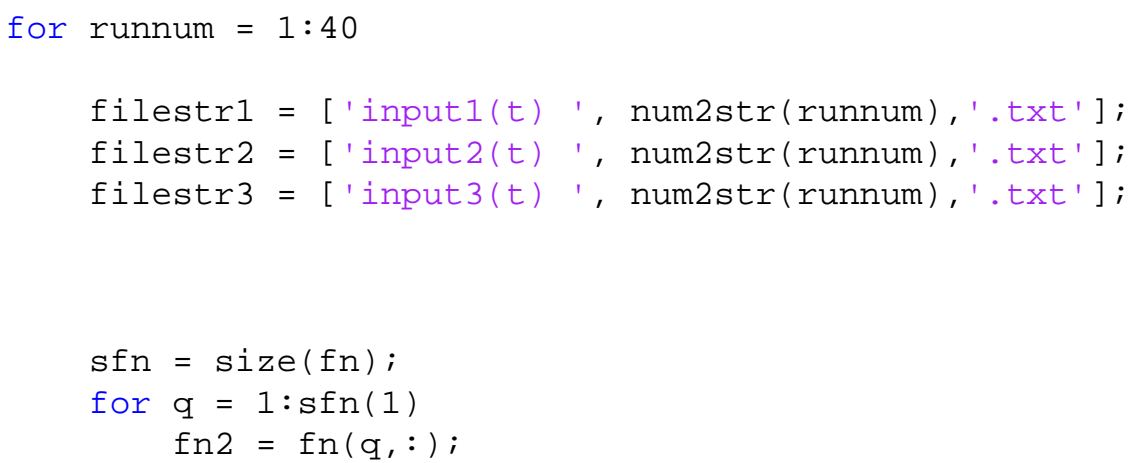


if $\operatorname{strcmpi(fn}(q,:)$, filestr 1$)==1$

\section{File Import}

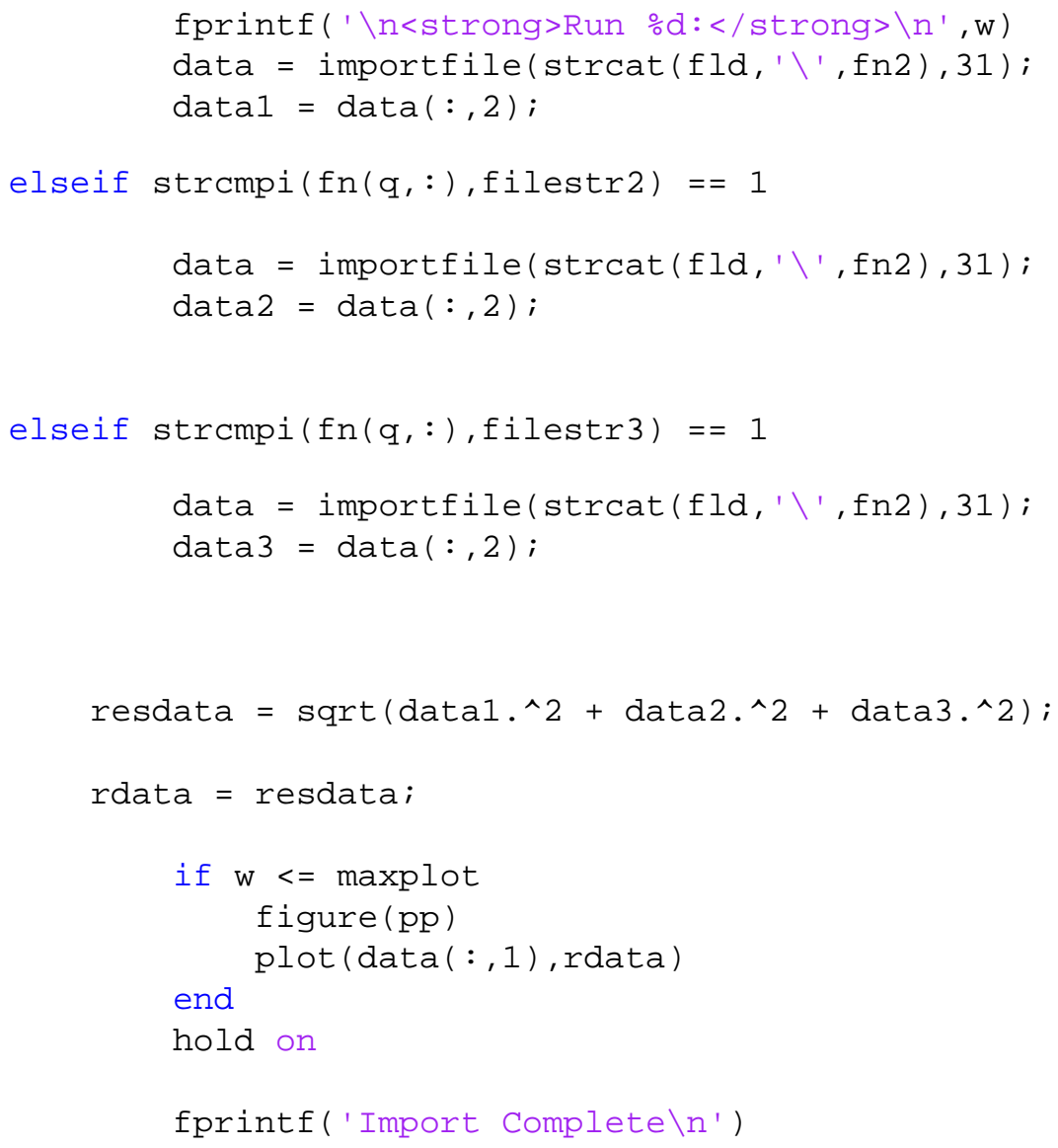

\section{Data filtering}

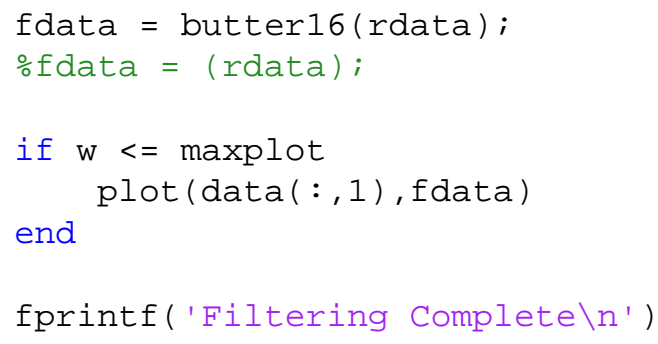

\section{Individual SI}

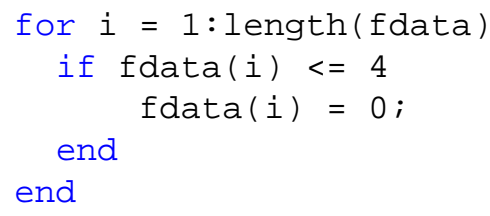




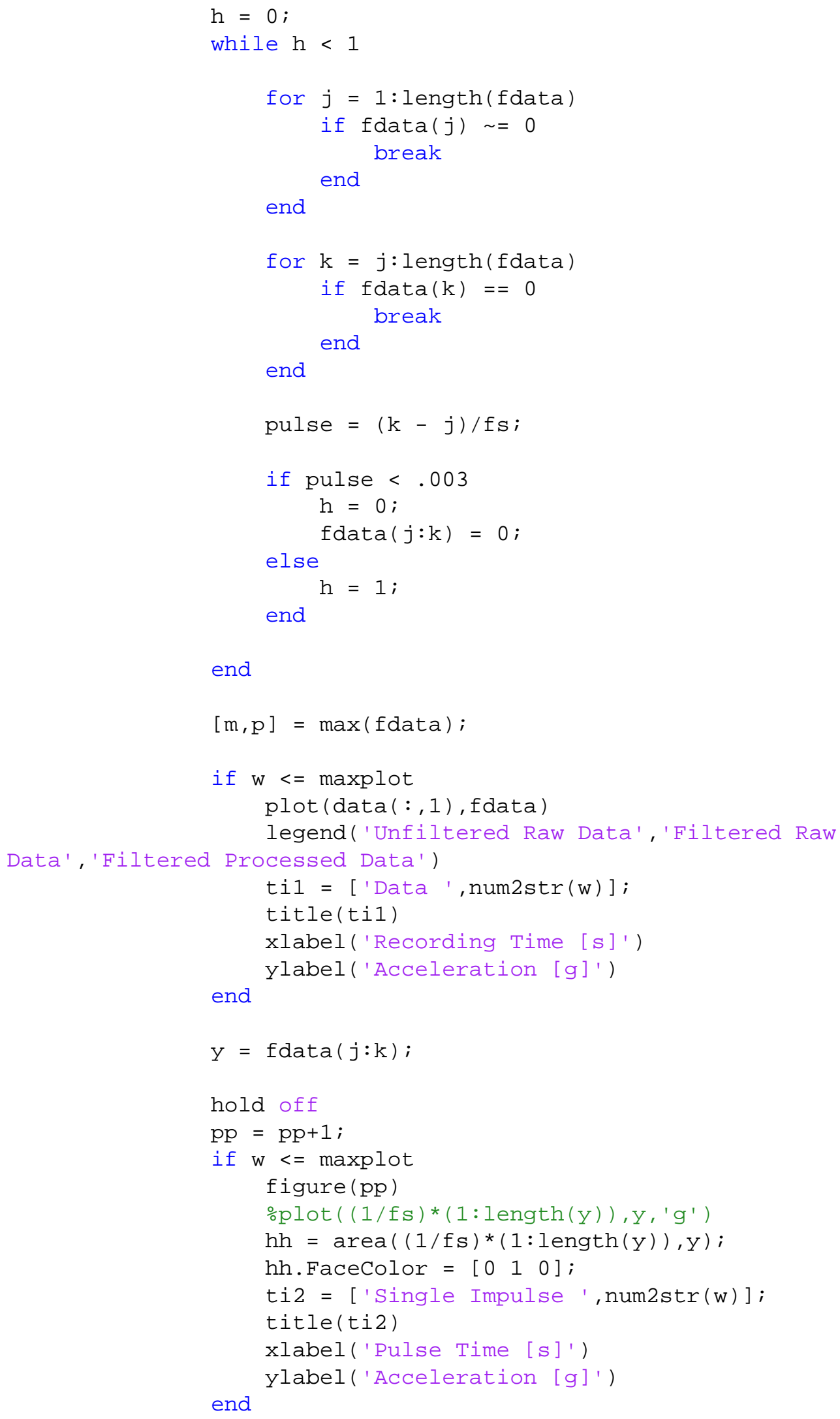




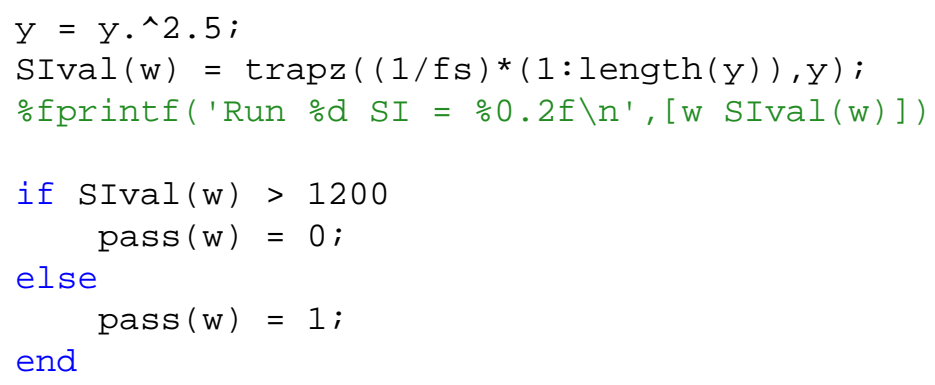

\section{Energy Dissipation Coefficient}

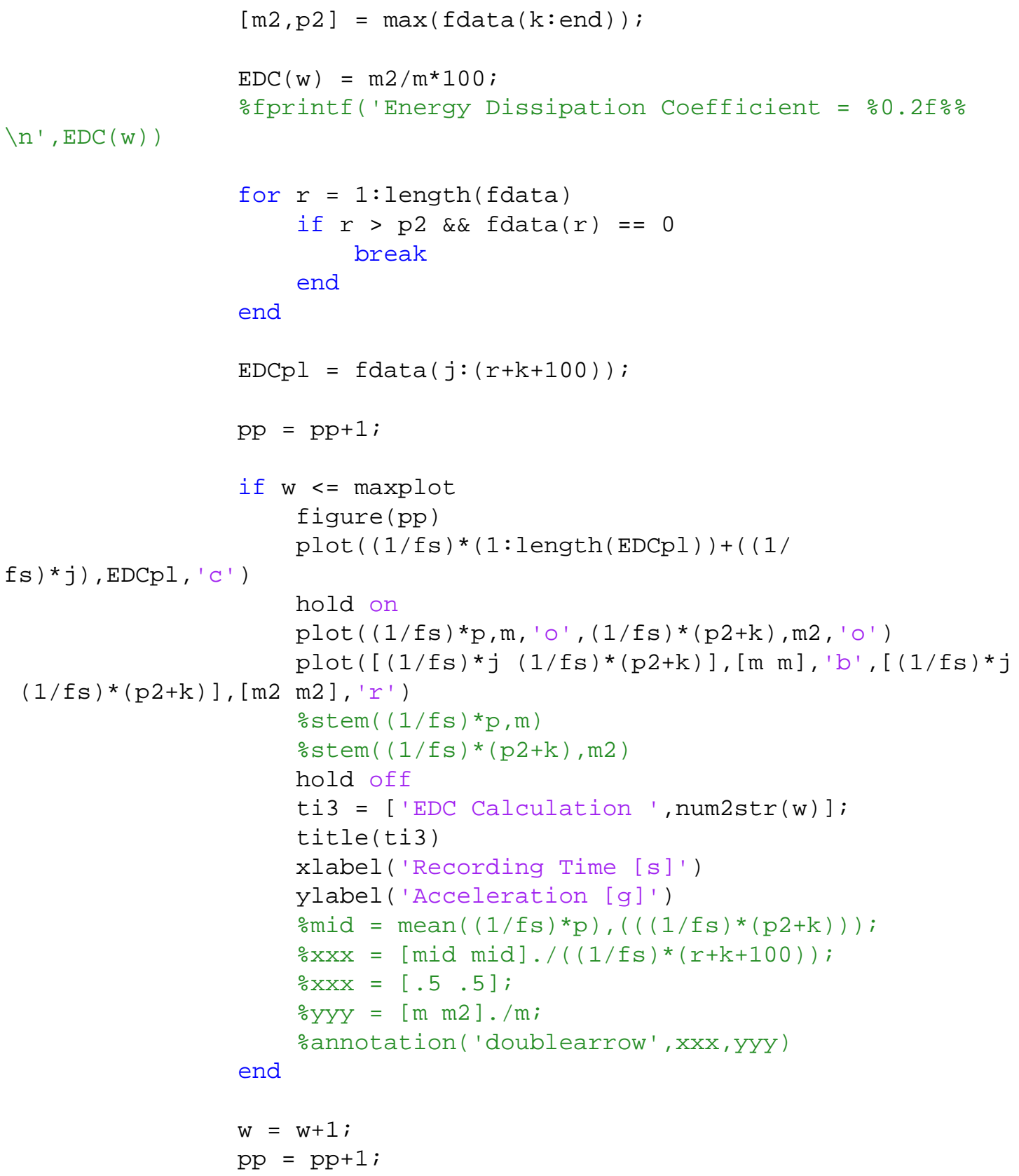


end

end

end

SImean $=$ mean (SIval);

EDCmean $=$ mean $(\mathrm{EDC})$;

fprintf (' $\backslash \mathrm{n} \backslash \mathrm{n}<$ strong $>$ Mean $\mathrm{SI}=\div 0.2 \mathrm{f}</$ strong $>\backslash \mathrm{n}$ ', SImean)

if $\min ($ pass) $\sim=1$

fprintf('Helmet Fails $\backslash n$ ')

else

fprintf ('Helmet Passes $\backslash n$ ')

end

fprintf('<strong $>$ Mean $\mathrm{EDC}=\frac{\circ}{0}$. 2 f\% $\%</$ strong $>\backslash \mathrm{n} \backslash \mathrm{n}^{\prime}$, EDCmean)

\section{Table Creation}

$\mathrm{T}=$ table ([1:(w-1) ] ', SIval',EDC', 'VariableNames', \{'Run', 'SI', 'EDC'\}); $\operatorname{disp}(\mathrm{T})$

writetable $(T, t b l)$

disp ('DONE')

Published with MATLAB® R2016a 


\section{Table of Contents}

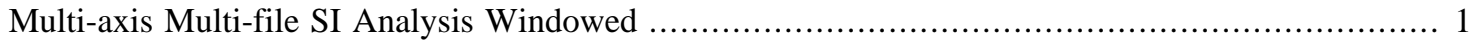

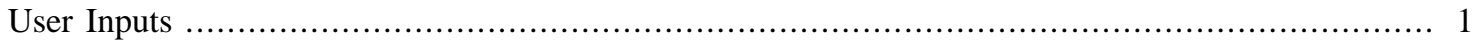

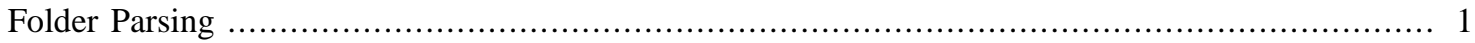

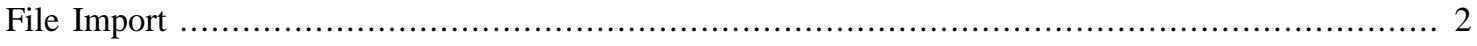

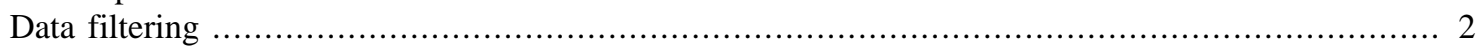

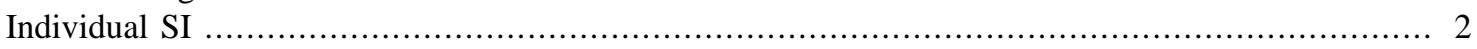

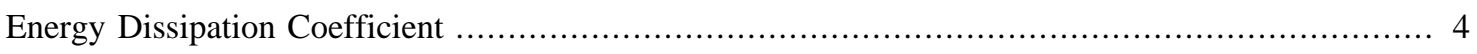

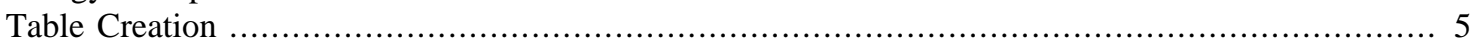

\section{Multi-axis Multi-file SI Analysis Windowed}

Michael Schuster 5/24/2016

analyses data to determine SI

clear all

clc

fprintf('Multi-axis Multi-file SI Analyzer $\backslash n$ ')

fprintf('Michael Schuster $\backslash n \backslash n$ ')

\section{User Inputs}

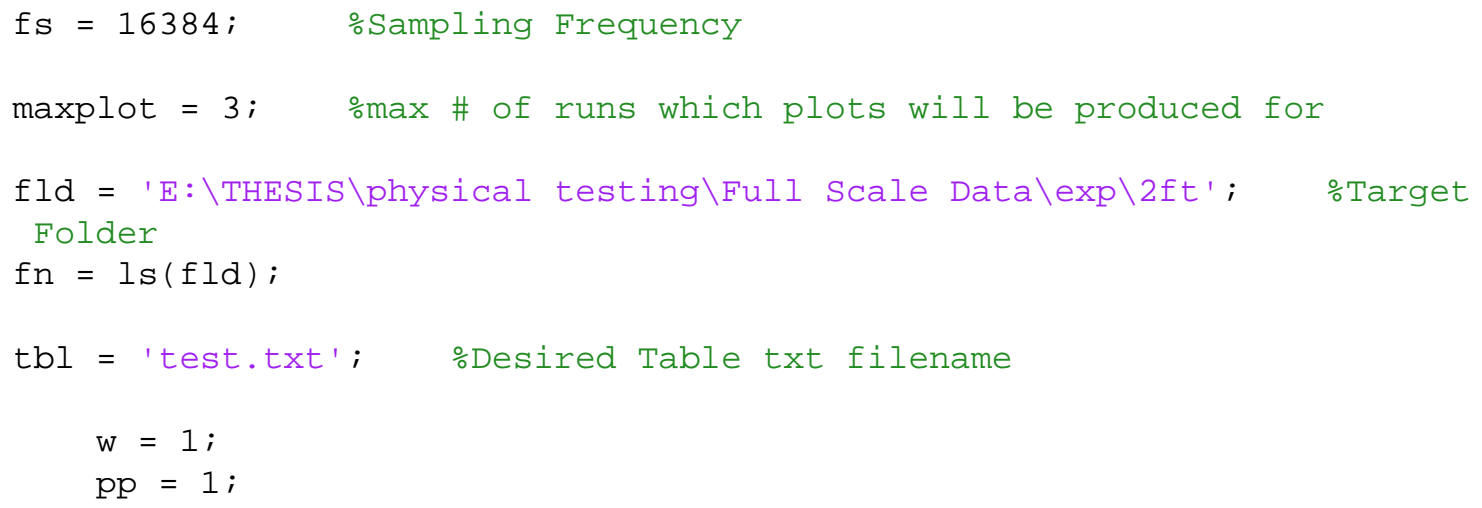

\section{Folder Parsing}

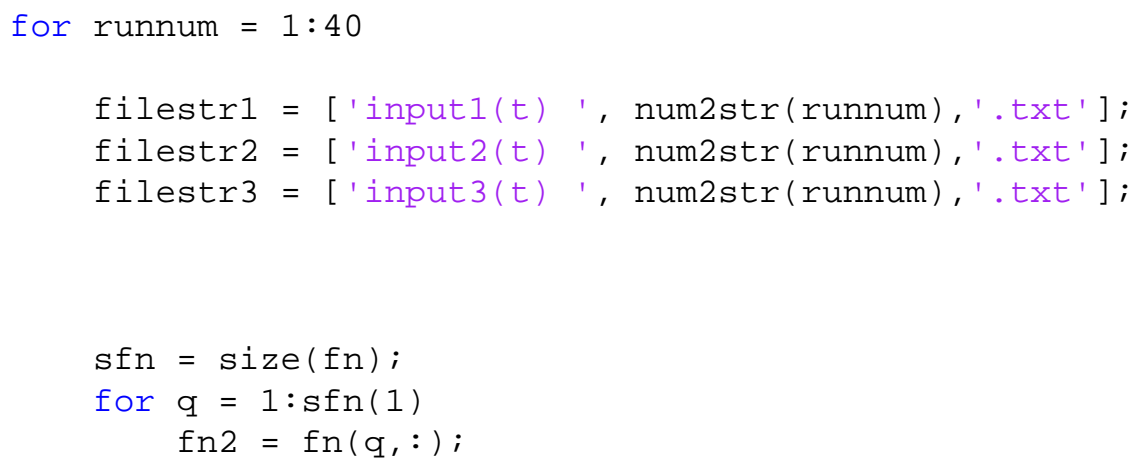


if $\operatorname{strcmpi(fn}(q,:)$, filestr 1$)==1$

\section{File Import}

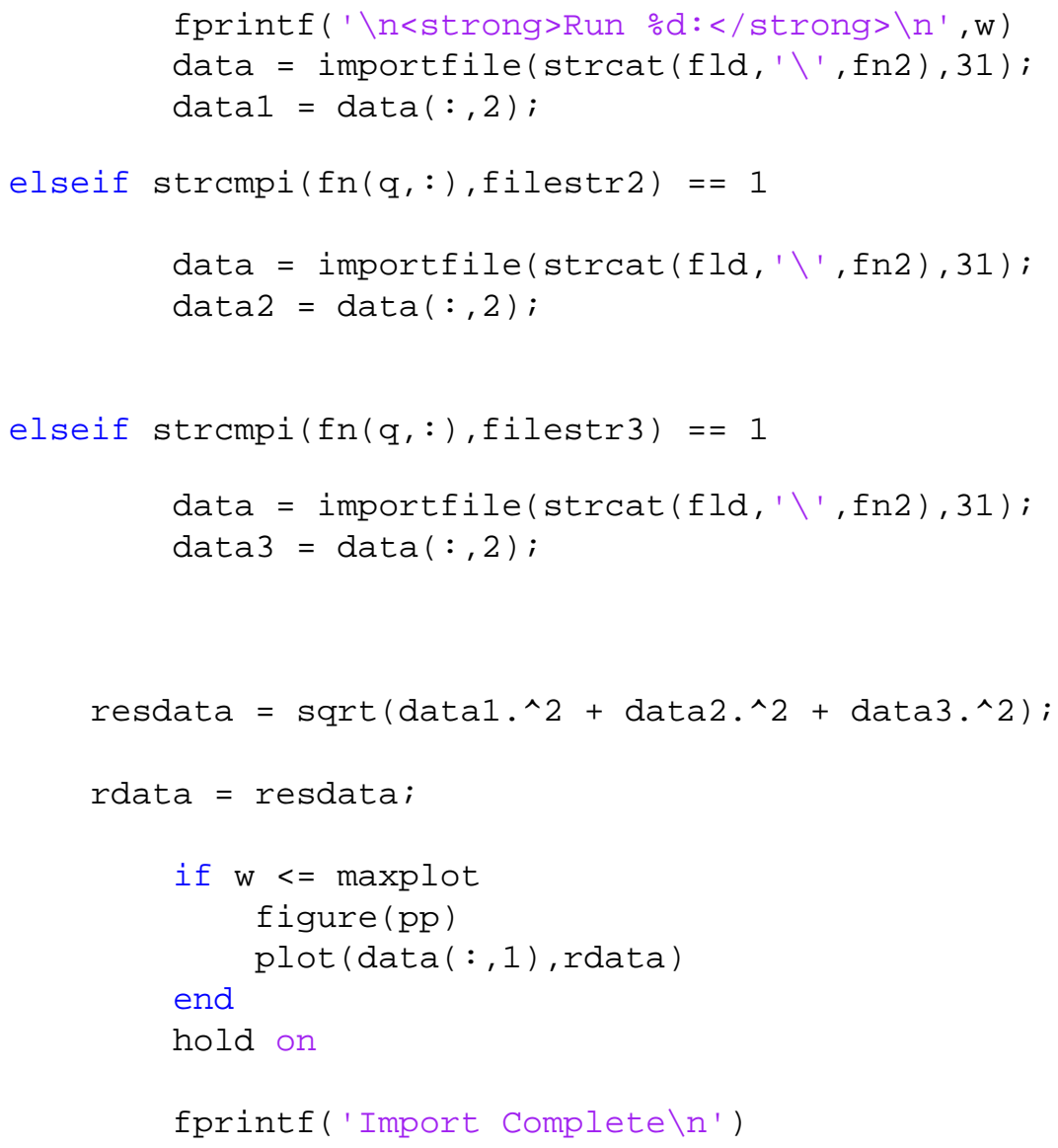

\section{Data filtering}

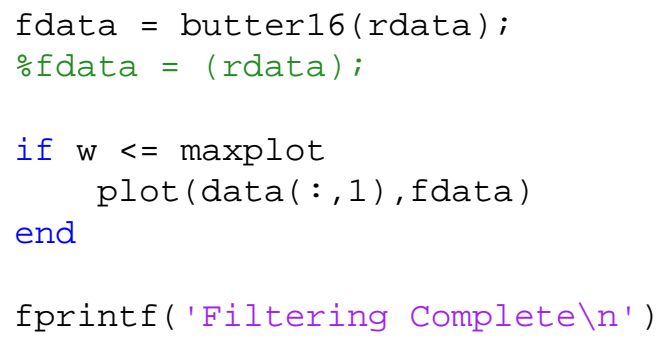

\section{Individual SI}

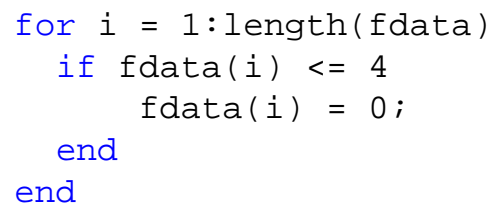




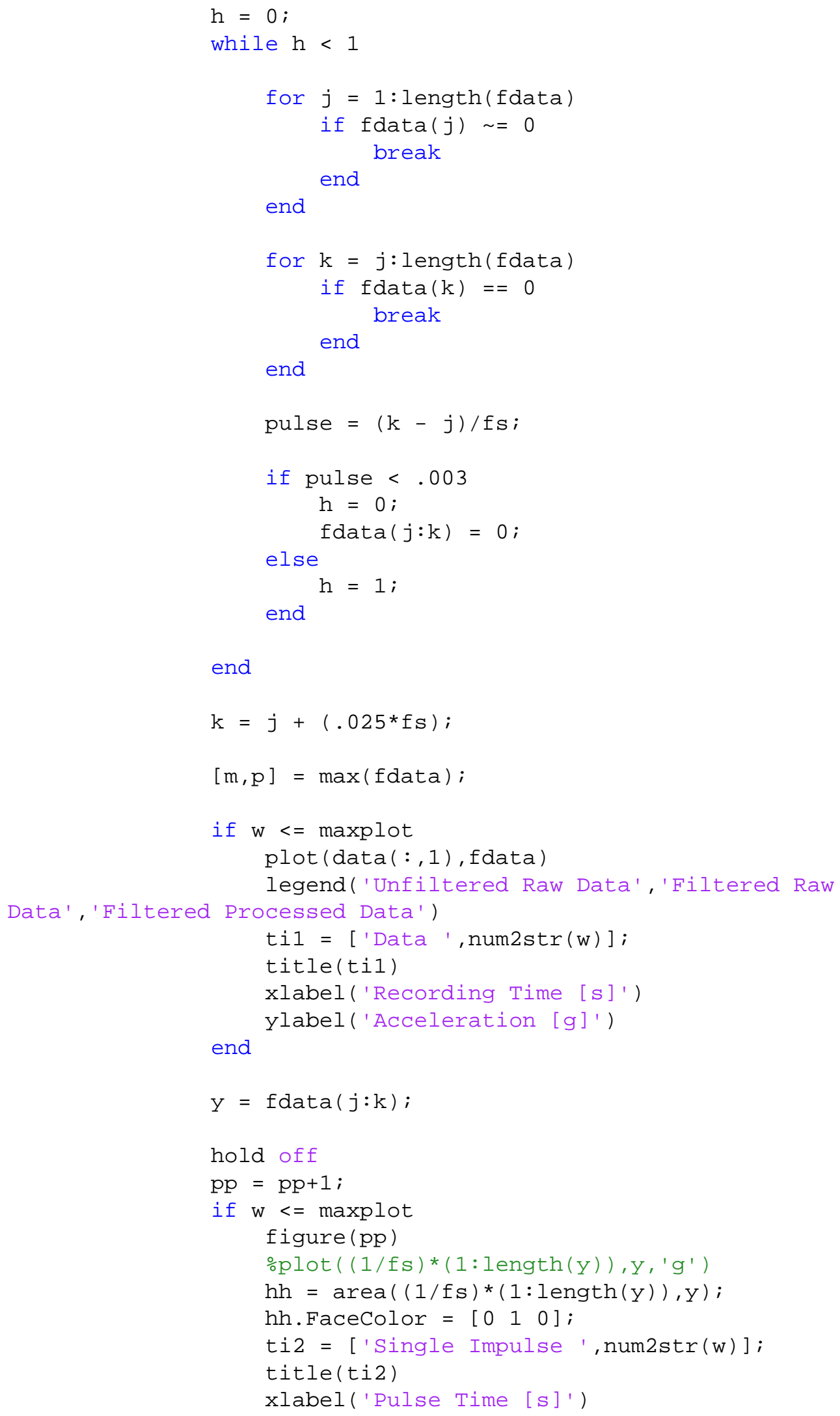




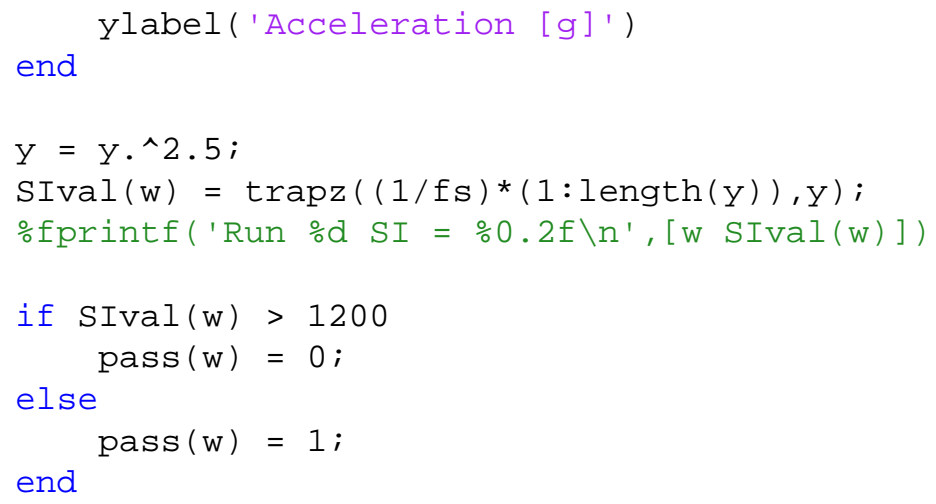

\section{Energy Dissipation Coefficient}

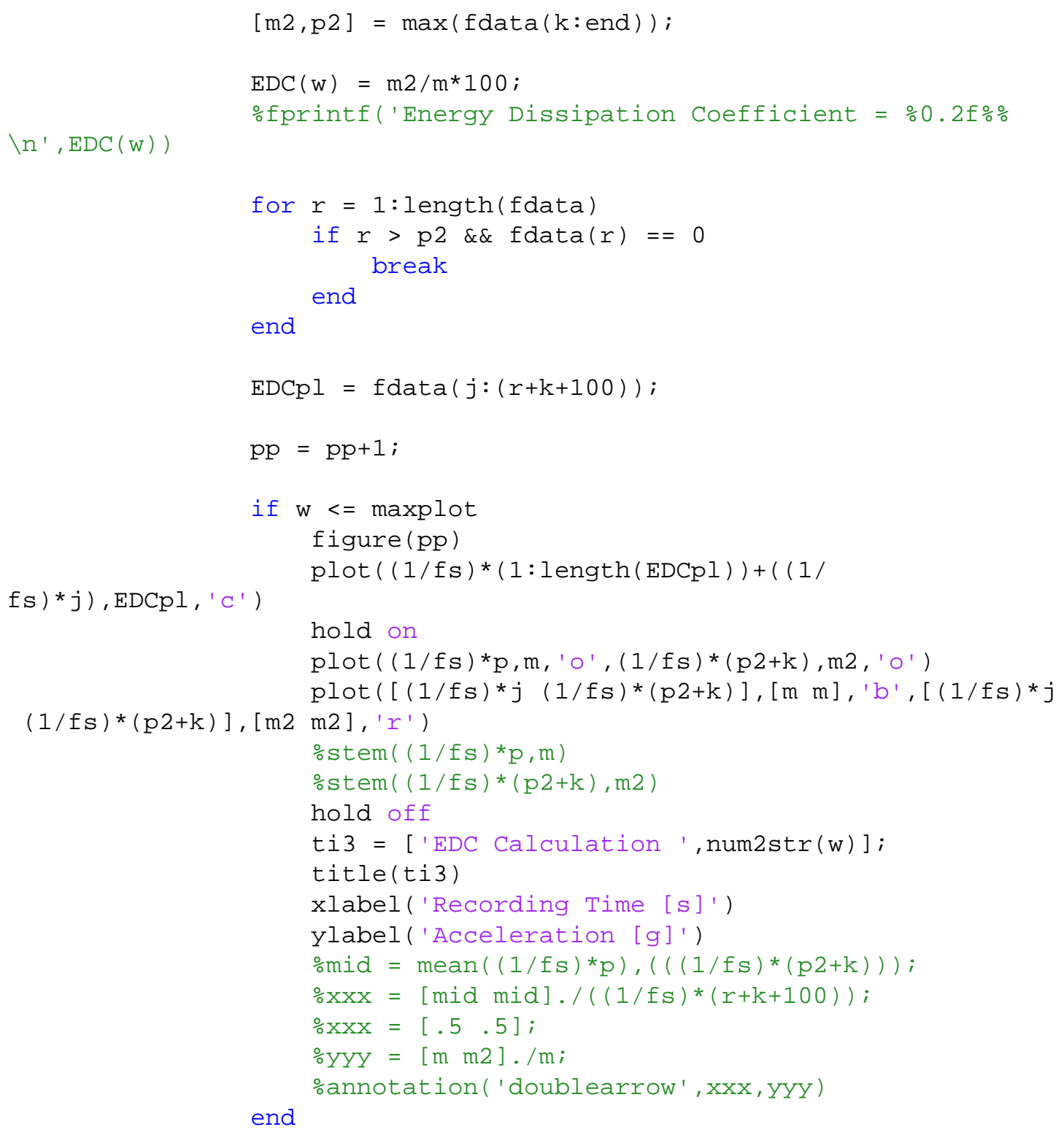




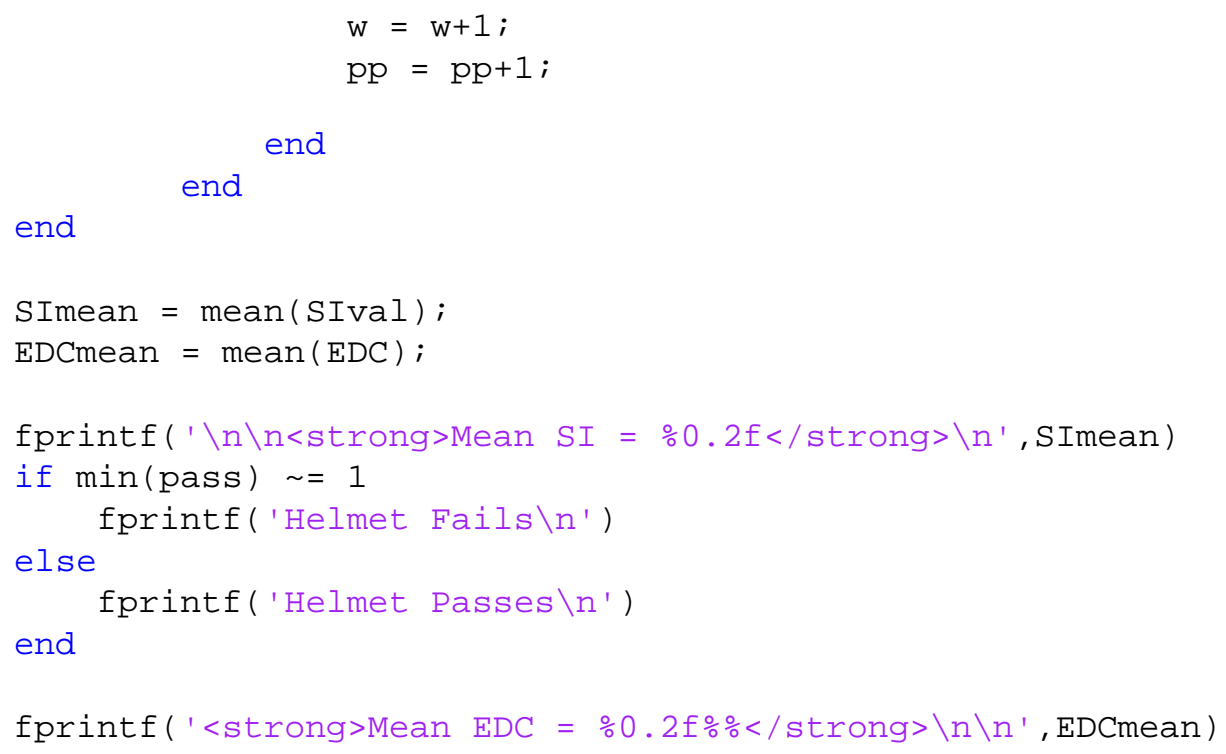

\section{Table Creation}

$\mathrm{T}=$ table([1:(w-1) ]', SIval',EDC', 'VariableNames', \{ 'Run', 'SI', 'EDC'\}); disp (T)

writetable ( $\mathrm{T}, \mathrm{tbl})$

disp ('DONE')

Published with MATLAB® R2016a 


\section{Table of Contents}

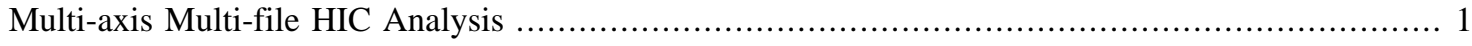

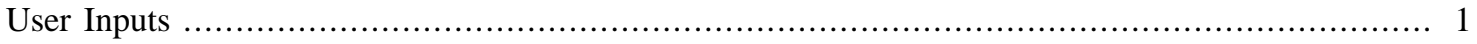

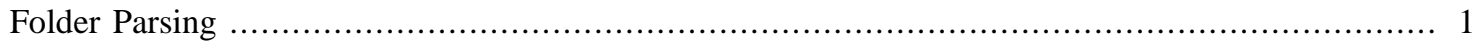

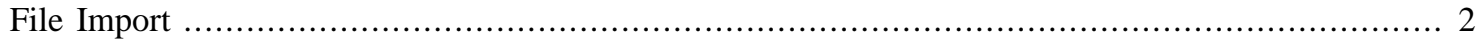

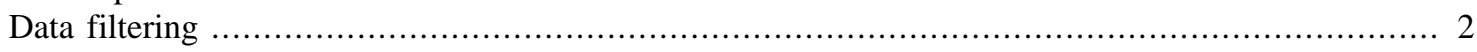

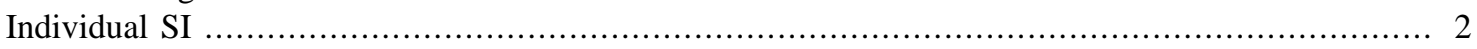

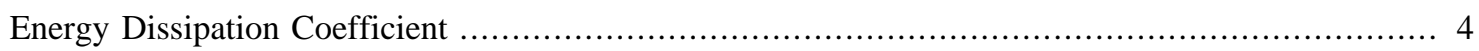

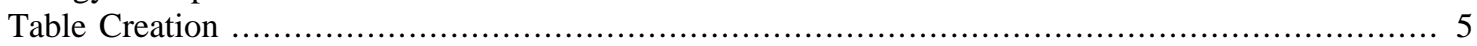

\section{Multi-axis Multi-file HIC Analysis}

Michael Schuster 5/24/2016

analyses data to determine SI

clear all

clc

fprintf('Multi-axis Multi-file SI Analyzer $\backslash n$ ')

fprintf('Michael Schuster $\backslash n \backslash n$ ')

\section{User Inputs}

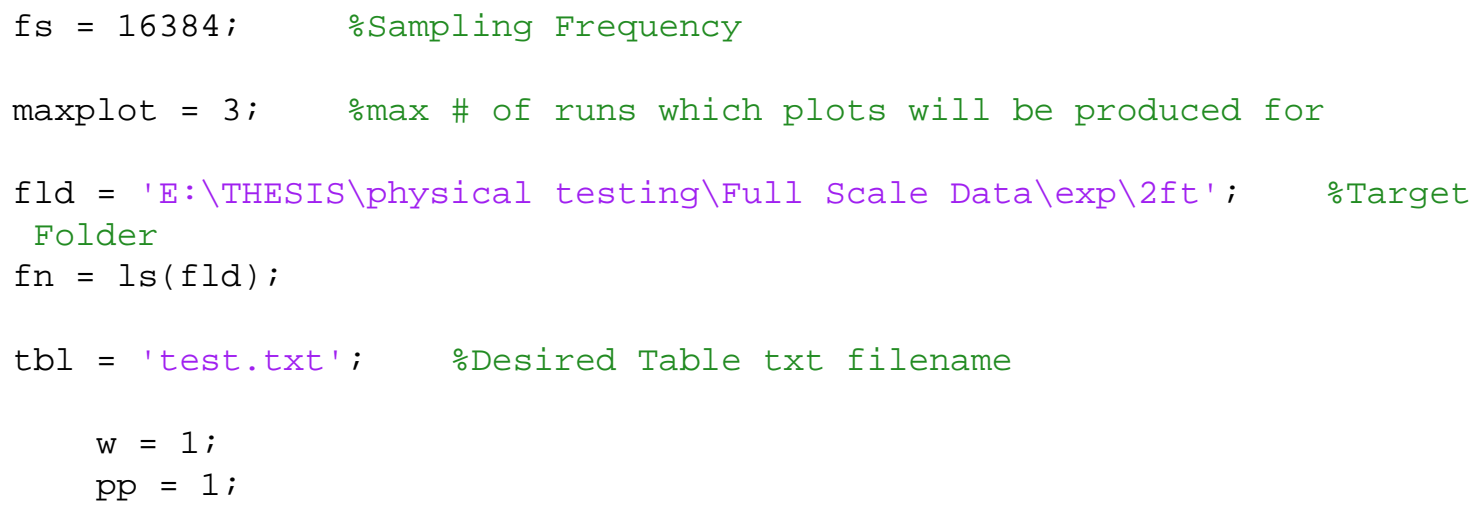

\section{Folder Parsing}

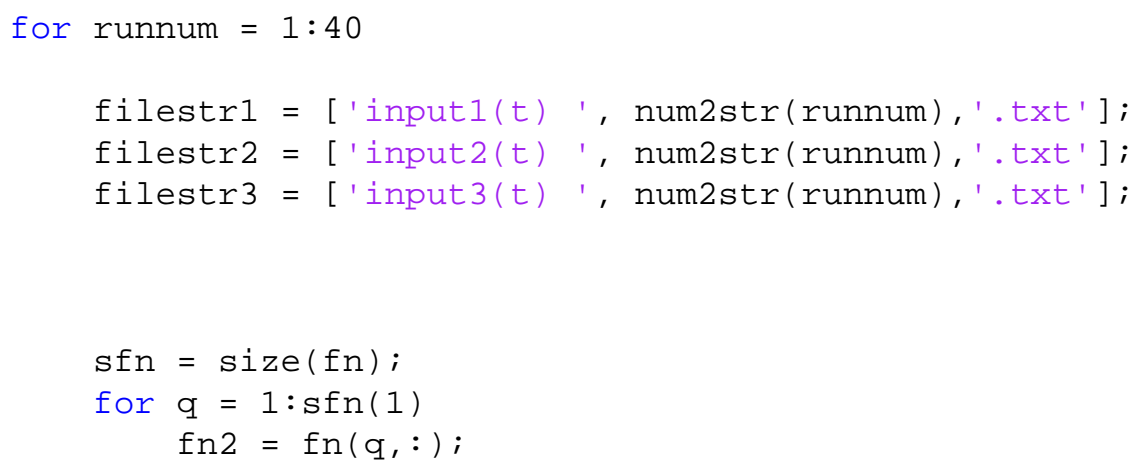


if $\operatorname{strcmpi(fn}(q,:)$, filestr 1$)==1$

\section{File Import}

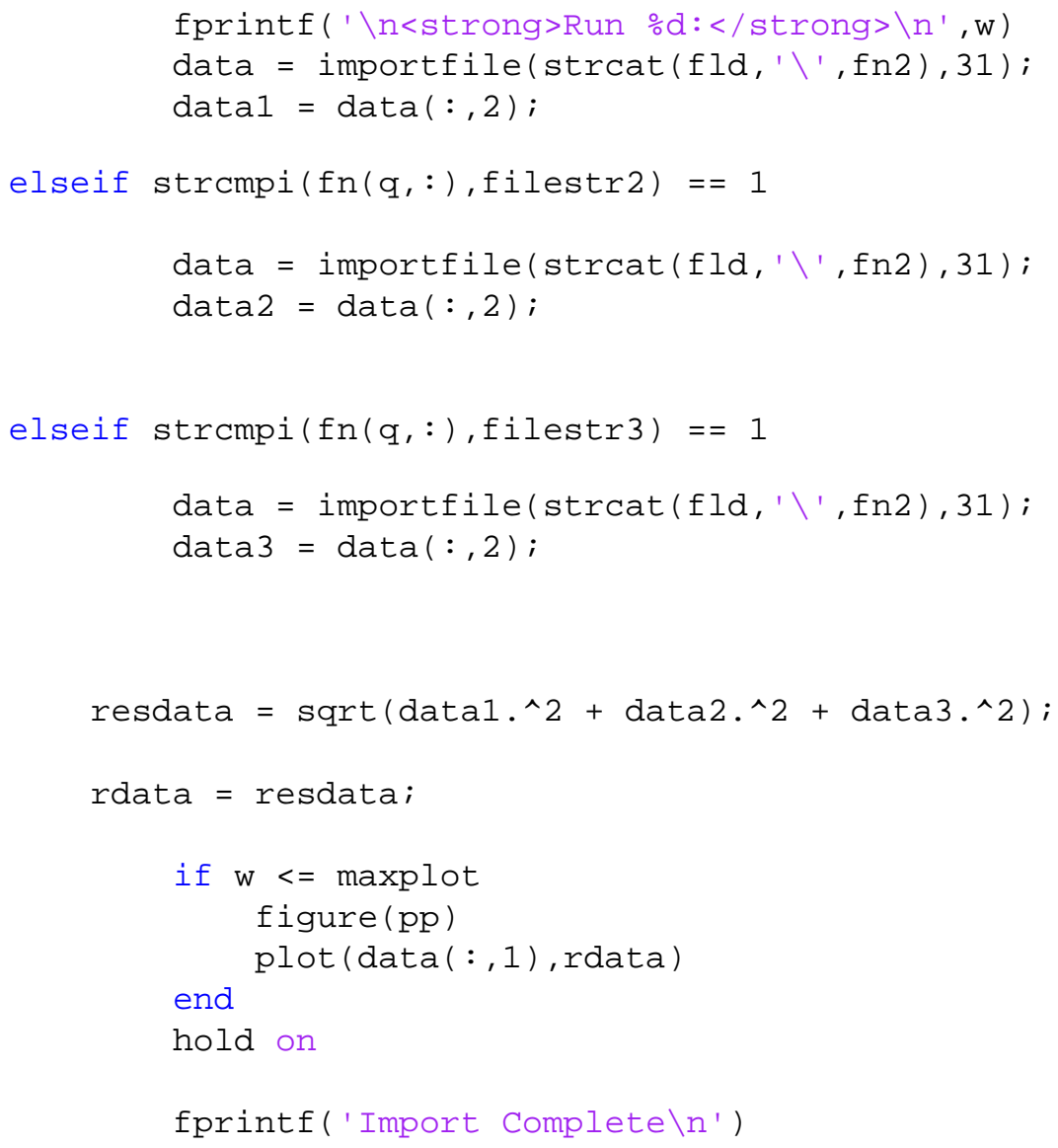

\section{Data filtering}

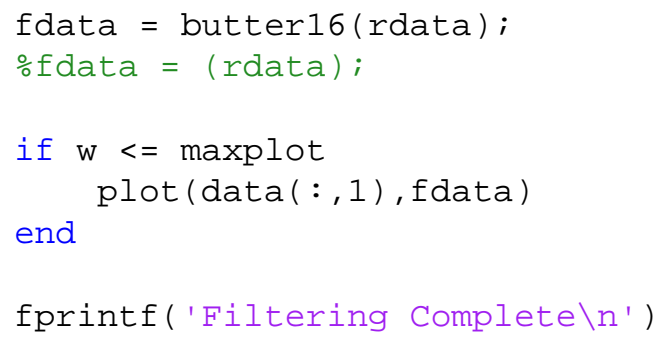

\section{Individual SI}

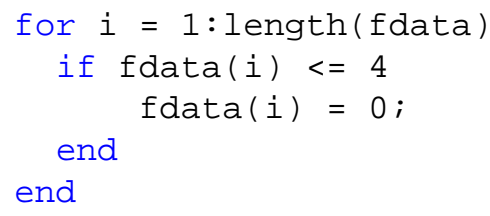




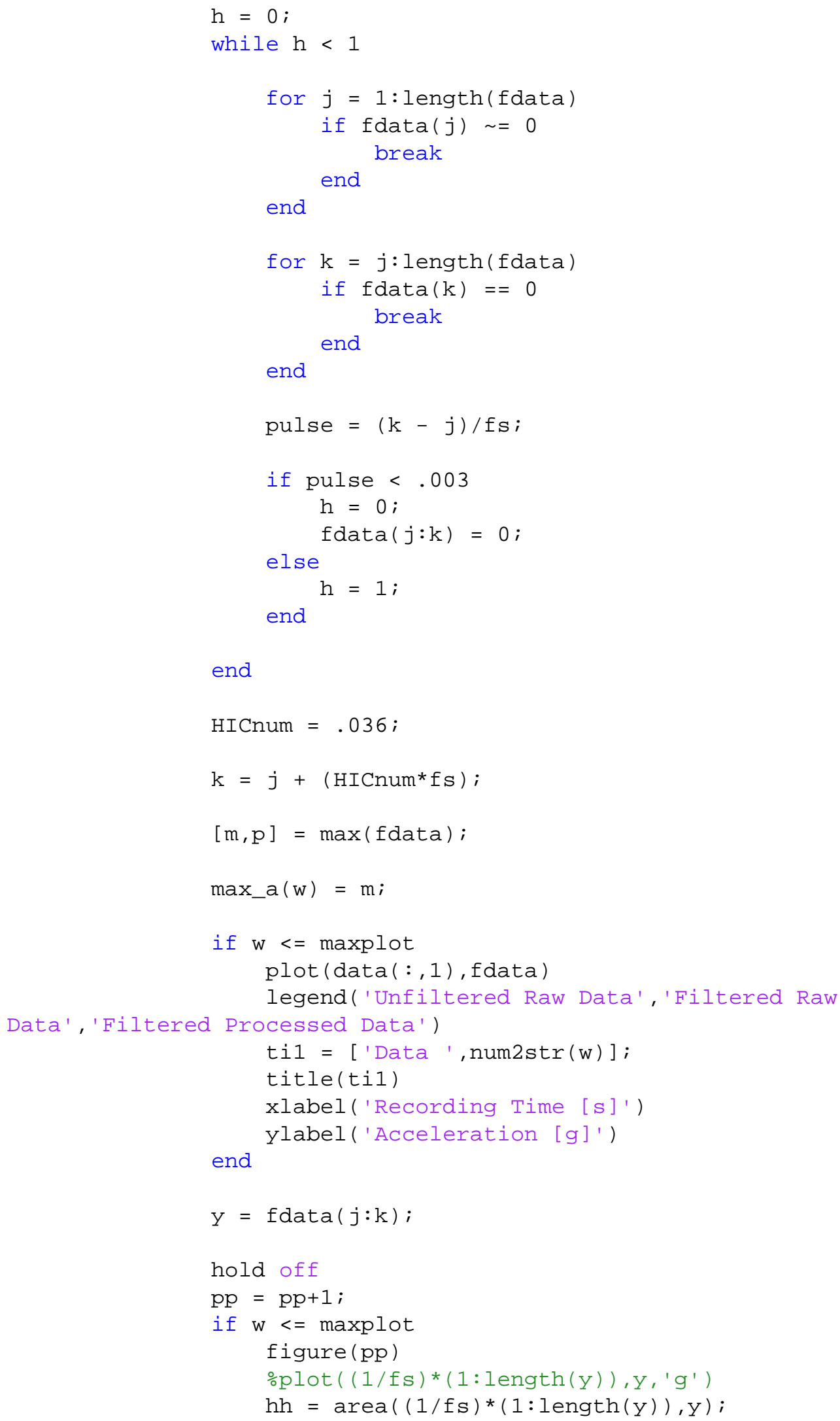




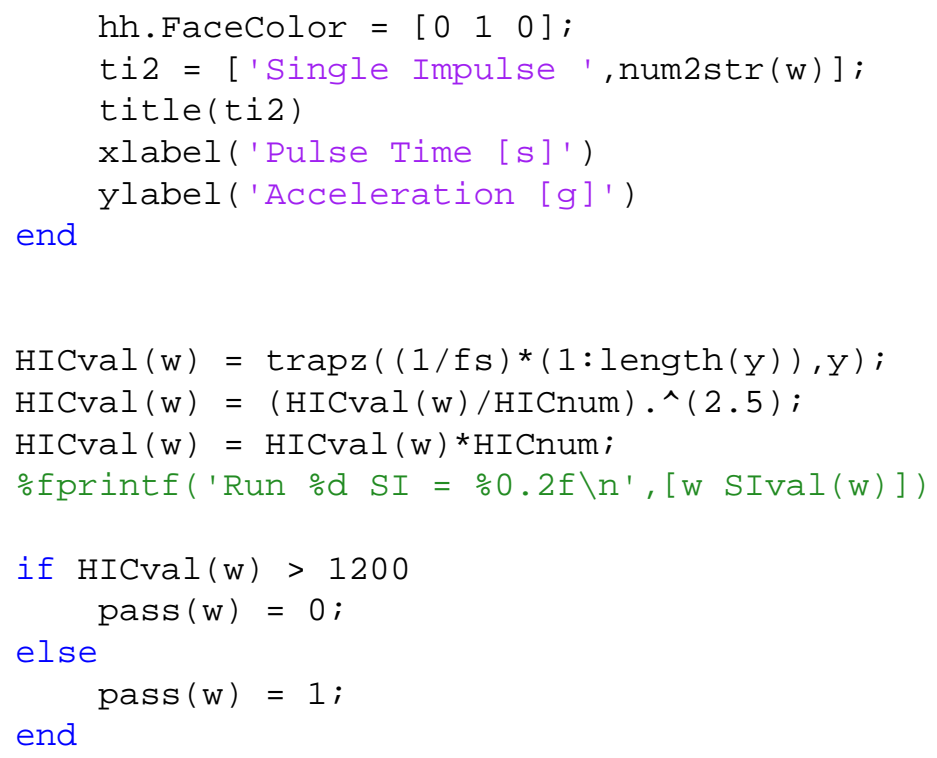

\section{Energy Dissipation Coefficient}

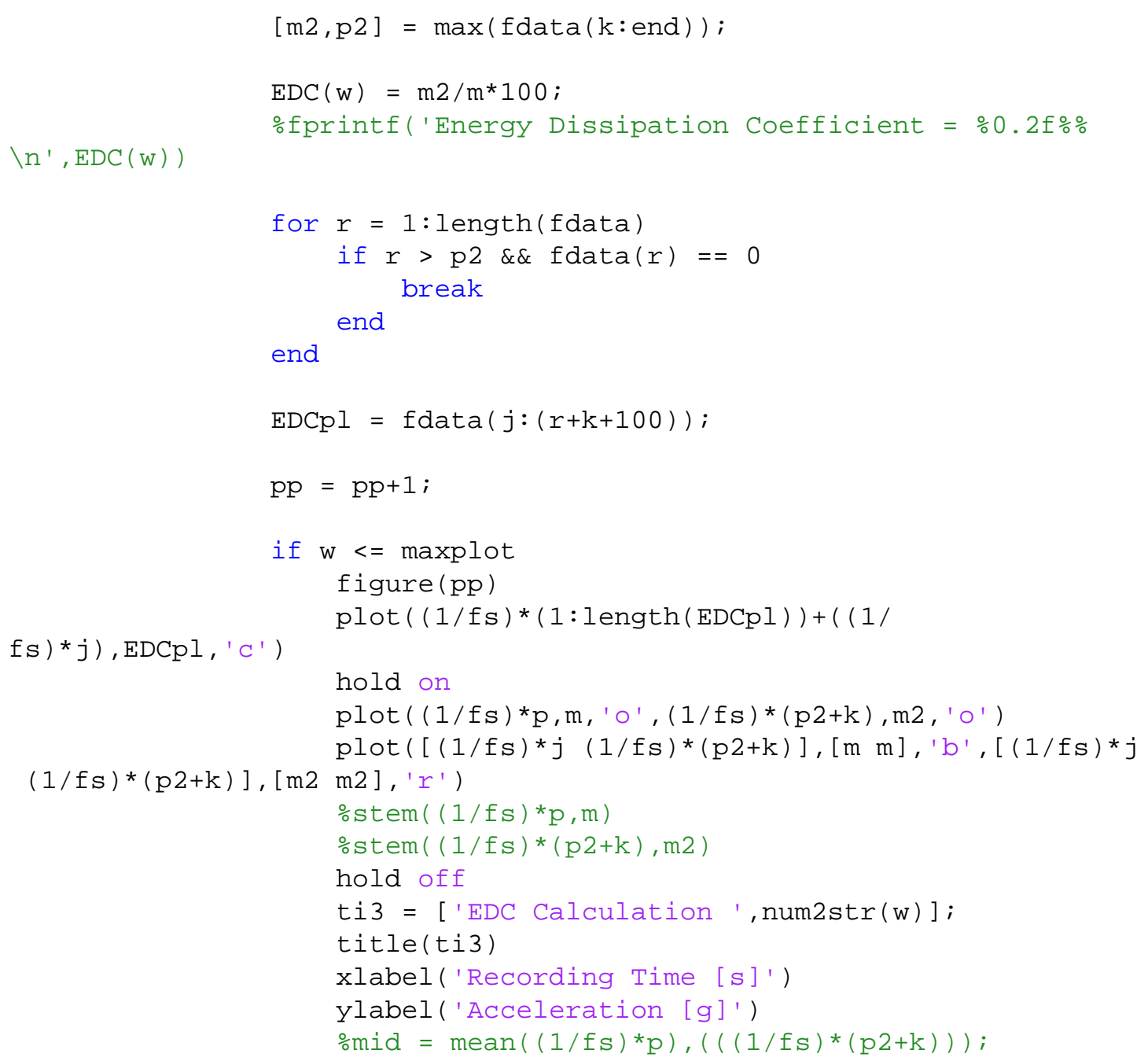




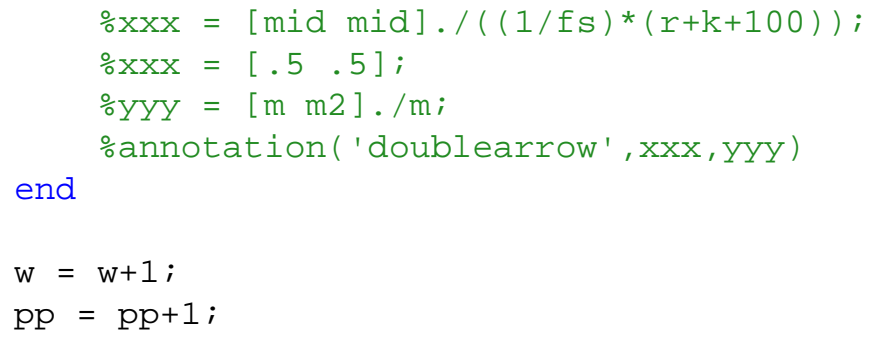

\section{Table Creation}

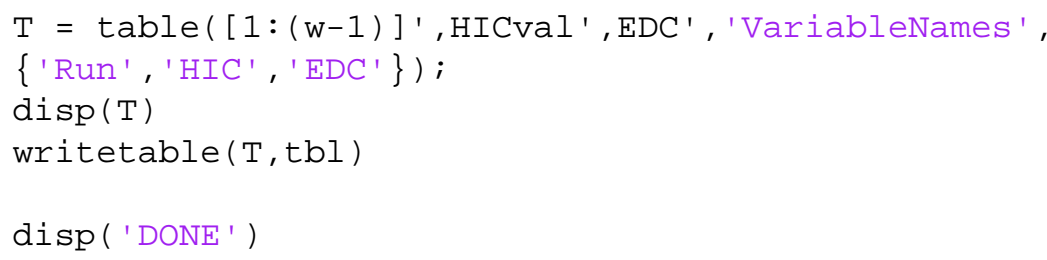

Published with MATLAB® R2016a 


\title{
Table of Contents
}

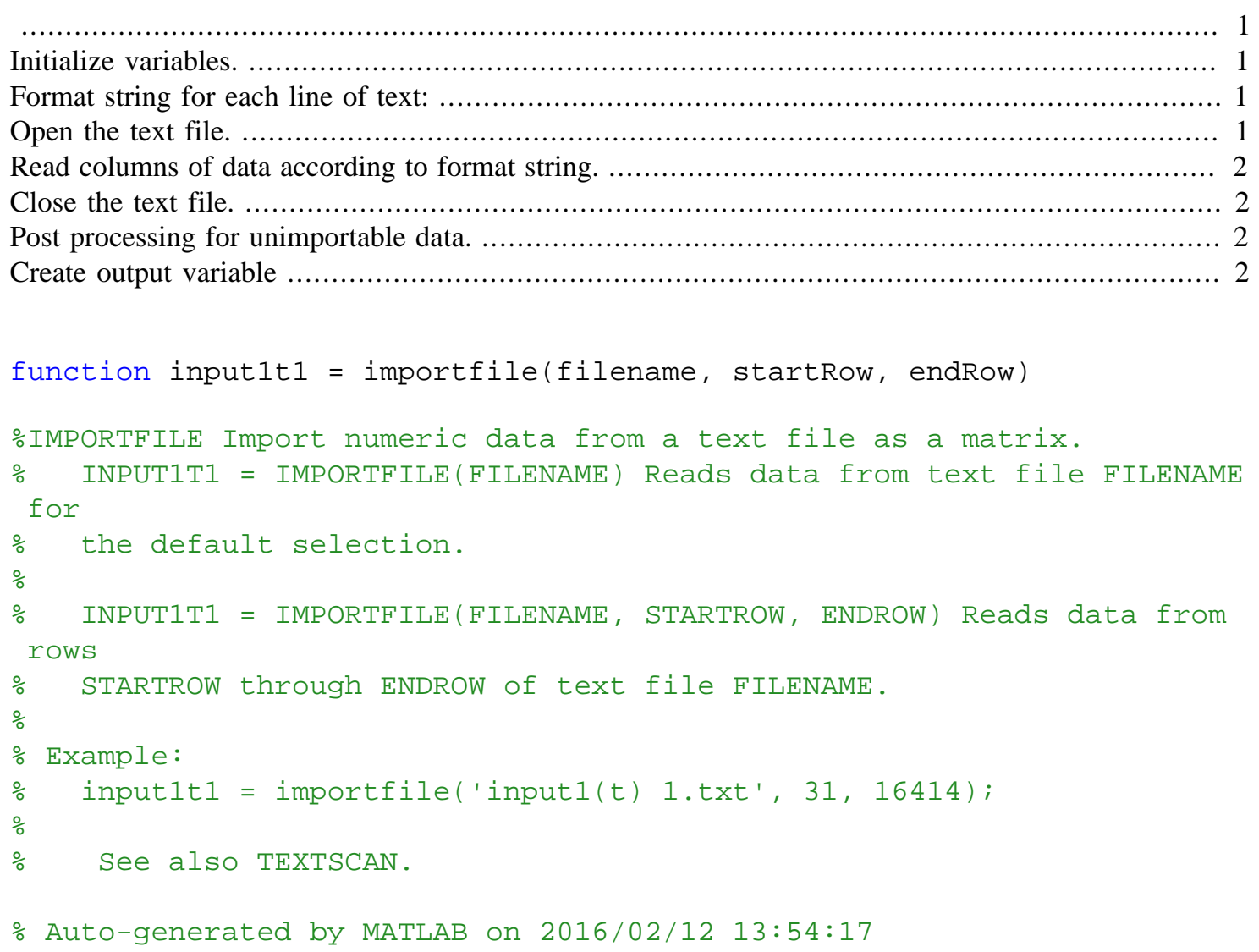

\section{Initialize variables.}

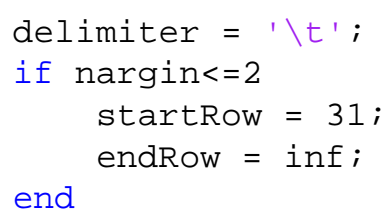

\section{Format string for each line of text:}

\author{
column 1 : double $(\% f)$ \\ \% column2: double (\%f) \\ \% For more information, see the TEXTSCAN documentation. \\ formatSpec $\left.={ }^{\prime} \circ \mathrm{f} \% \mathrm{f} \%\left[{ }^{\wedge} \backslash \mathrm{n} \backslash \mathrm{r}\right]\right]^{\prime}$;
}

\section{Open the text file.}

fileID = fopen(filename, 'r'); 


\section{Read columns of data according to format string.}

This call is based on the structure of the file used to generate this code. If an error occurs for a different file, try regenerating the code from the Import Tool.

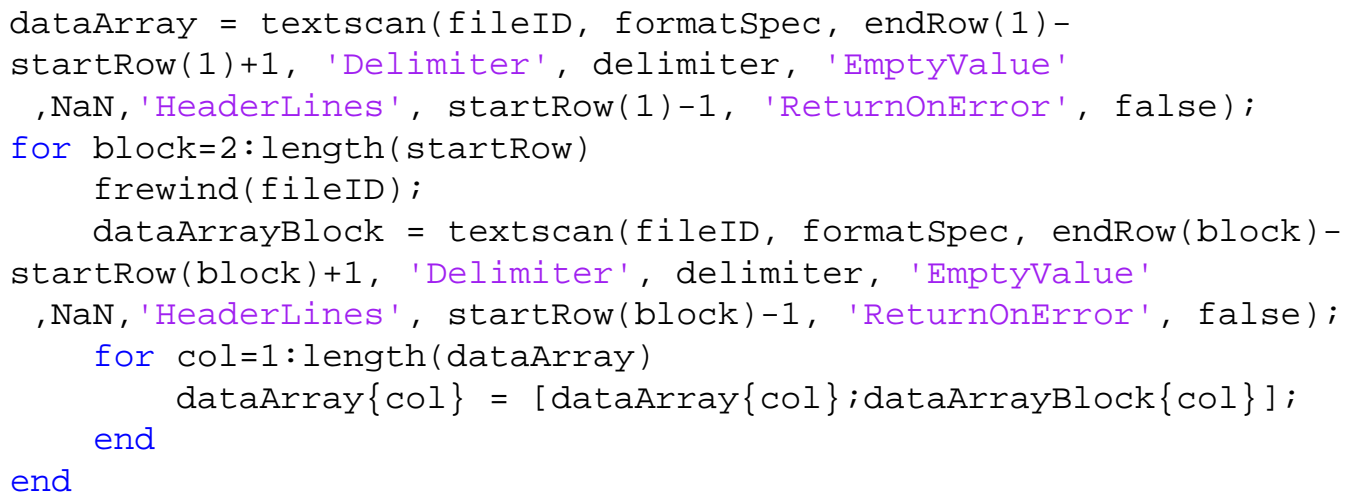

Close the text file.

fclose (fileID);

\section{Post processing for unimportable data.}

No unimportable data rules were applied during the import, so no post processing code is included. To generate code which works for unimportable data, select unimportable cells in a file and regenerate the script.

\section{Create output variable}

input 1 t $1=[$ dataArray $\{1:$ end-1 $\}]$;

Published with MATLAB® R2016a 


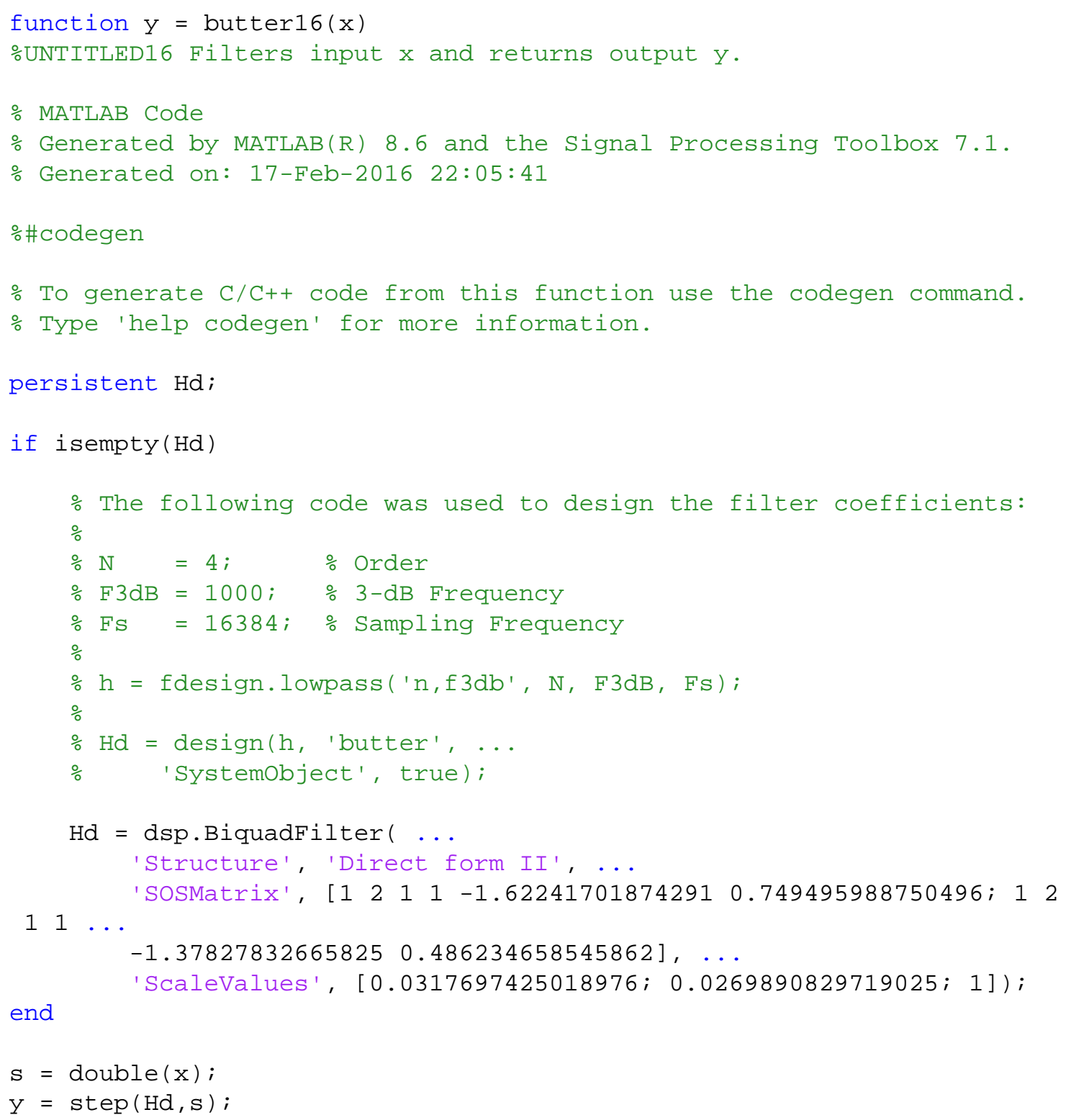

Published with MATLAB® R2016a 
APPENDIX F: SPECIFICATION SHEETS 


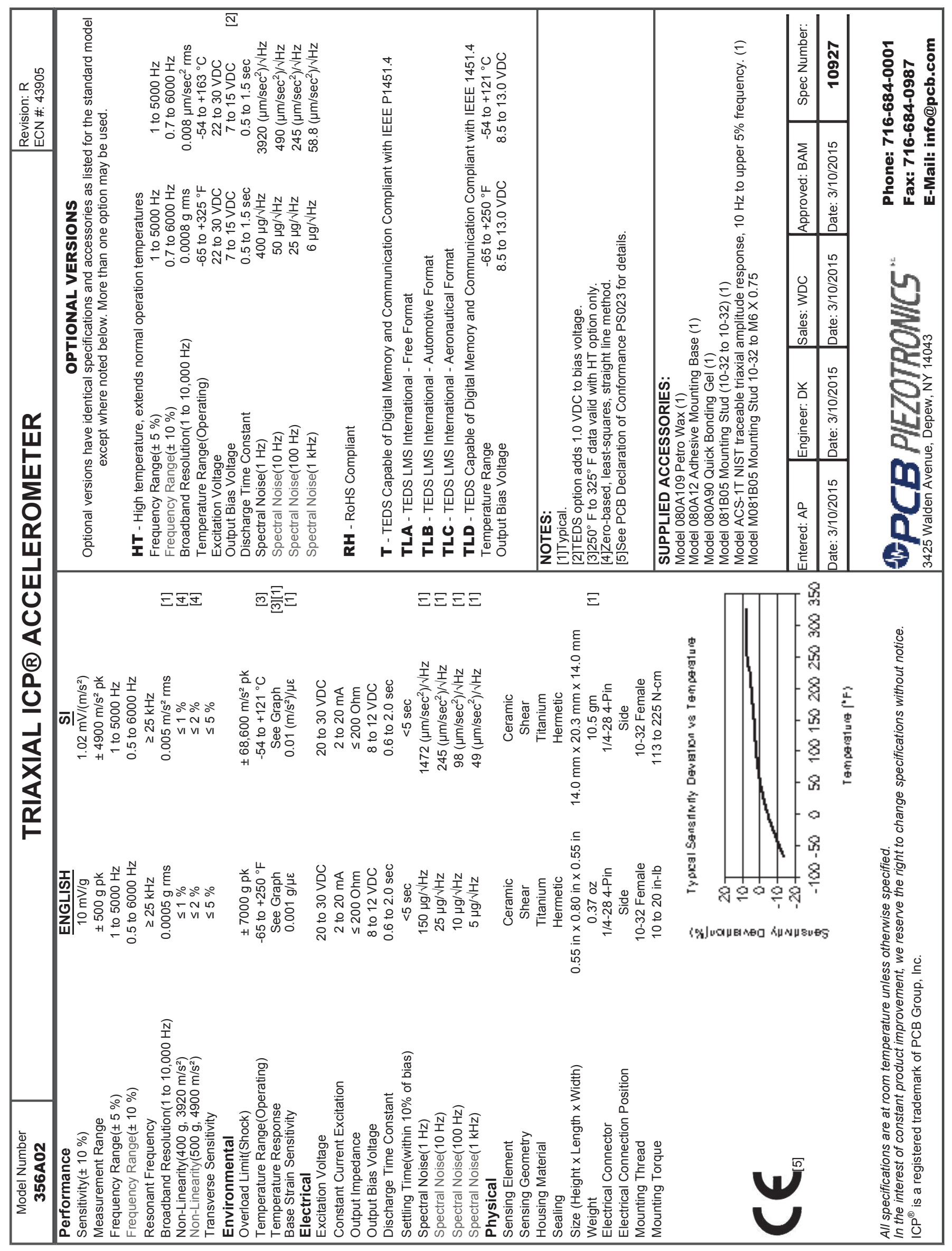




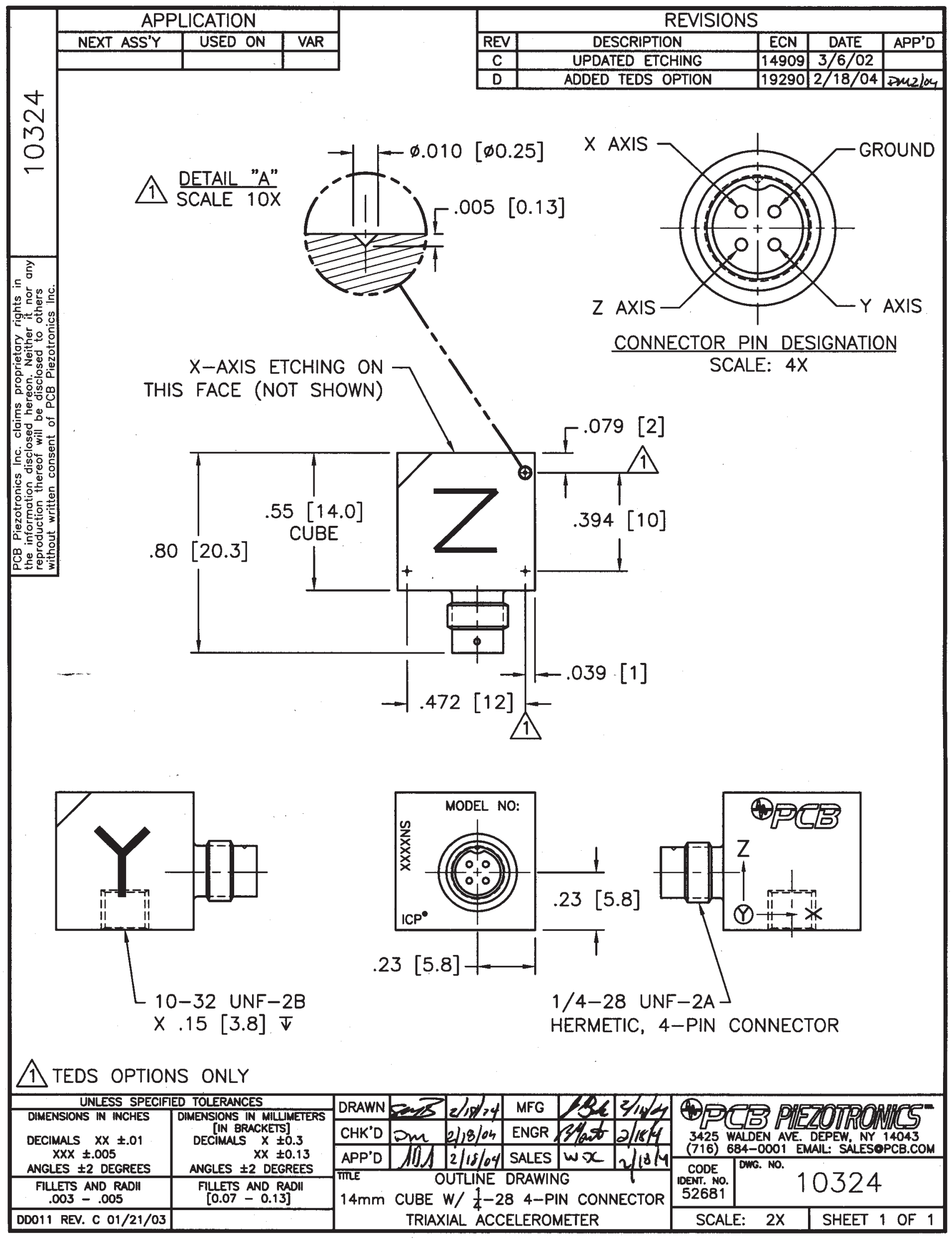




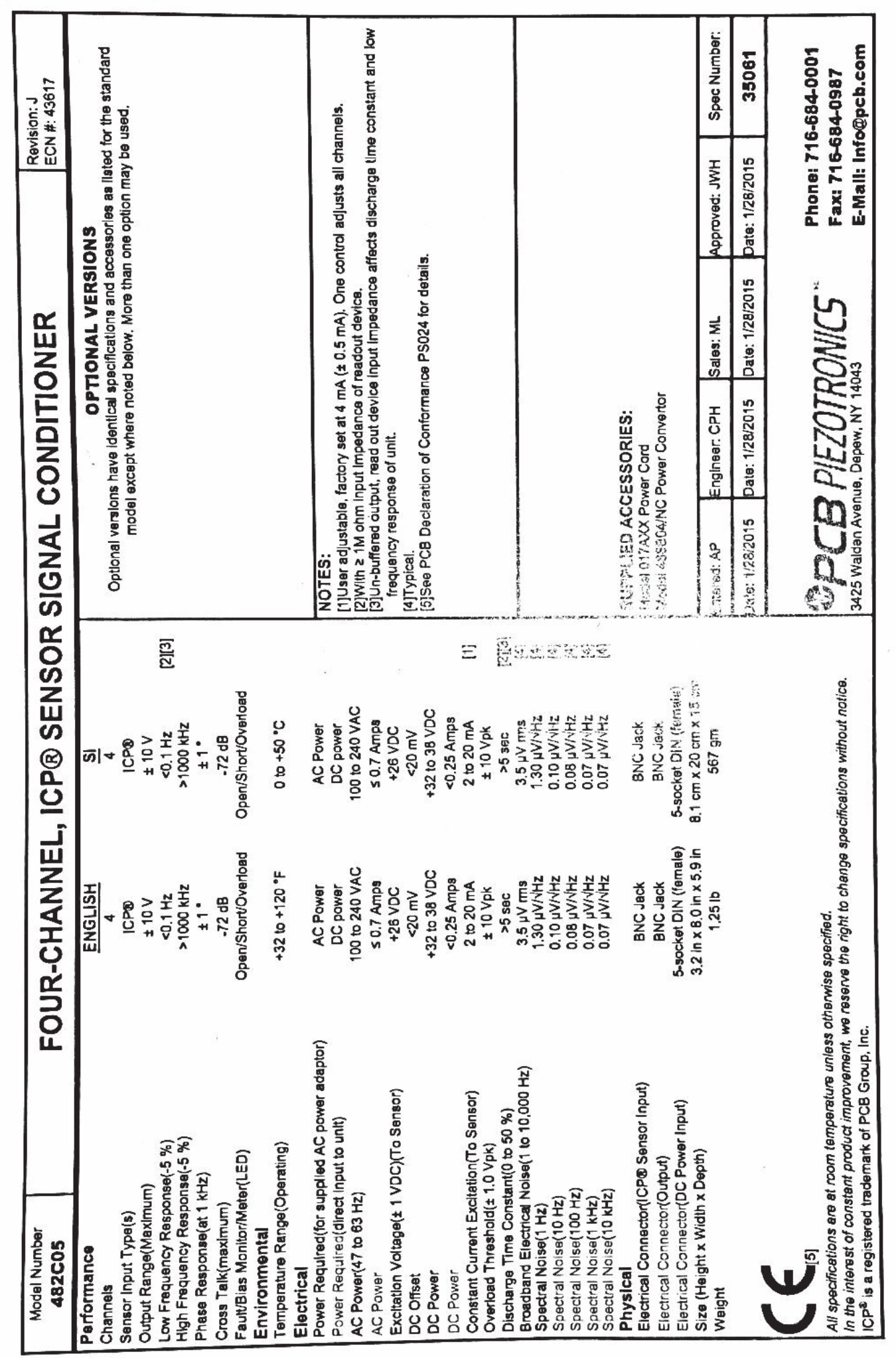




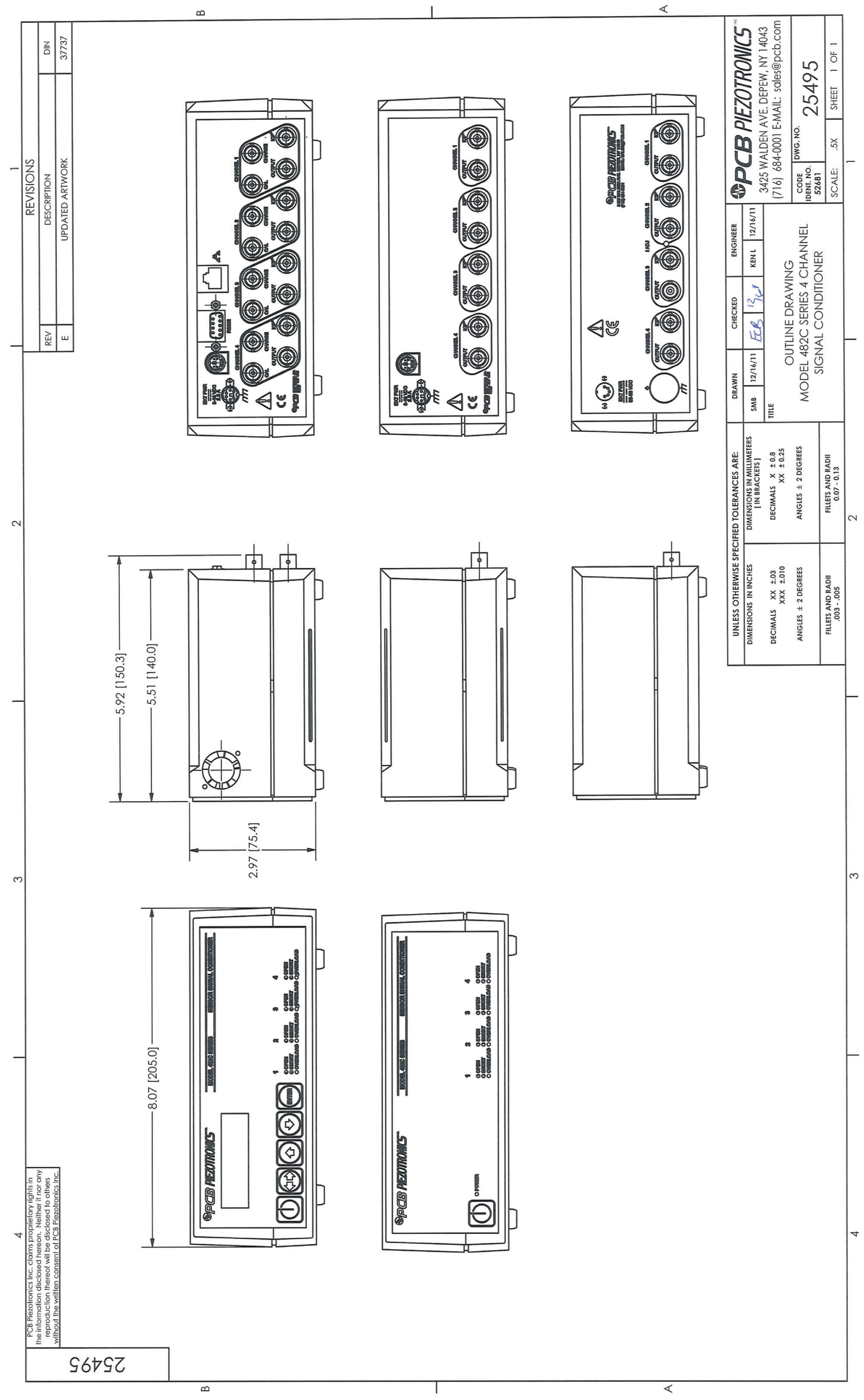


Real-time Analyzer with High-speed USB 2.0 Interface for Vibration and Noise Analysis Dynamic Signal Analyzer 


\section{FOCUS II}

\section{Tech n ic a I S p e c i f ic a t ions}

FOCUS II Real-time Signal Analyzer

2

RT Pro Signal Analysis and Waveform Source Software

3

RT Pro Signal Analysis Series

Modal Data Acquisition, Long Waveform Recorder Option, analyze Anywhere Option, Environmental Data Reduction Option, and Automated Test Option

\section{4}

\section{RT Pro Signal Analysis Series}

Acoustic Analysis Option, Advance Graphics Option,

RMP Spectrum Processing Option, and Realtiem Order Tracking Option

5

RT Pro Signal Analysis Series

Dactron Rotate Option and Dactron Rotate Plus Option 


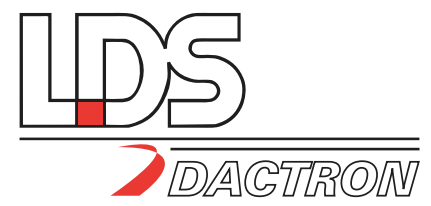

\section{FOCUS II Real-time Signal Analyzer}

FOCUS IT $T^{\mathrm{M}}$ makes any PC an instrument-quality analyzer that is a flexible multi-channel analyzer for noise and vibration analysis. Designed for realtime signal analysis, FOCUS II offers remarkable performance with a measurement dynamic range of $120 \mathrm{~dB}$ and a $42 \mathrm{kHz}$ realtime rate. FOCUS II is a USB device that is fully compliant with USB version 2.0, 1.1, and 1.0 specifications.

Signal analysis applications are available for the range of noise and vibration testing - realtime spectrum analysis, modal data acquisition, realtime octave analysis, order tracking and waterfall analysis, transient capture and SRS analysis. FOCUS // comes standard with four inputs with ICP ${ }^{\circledR}$ sensor power, all housed in an steel/aluminum case with expansion bays to suport up to twenty inputs, a 6 foot (2.8 meter) USB cable, a user manual (on CD), and a one year warranty.

\section{Inputs}

Analog channels

Electronics

Filtering

Frequency range

Voltage range

Signal conditioning

Maximum input

Resolution

Dynamic range

Accuracy

Channel match

Amplitude

Phase

Alias protection

Signal-to-noise

Cross-talk

Total Harmonic

Distortion

Frequency accuracy

\section{Outputs}

\section{Analog channels} Electronics

Filtering

Frequency range

Voltage range

Resolution

Dynamic range

Total Harmonic

Distortion

Output impedance

Maximum current
Four standard, expandable to twenty simultaneous channels. All are differential inputs with $220 \mathrm{k} \mathrm{Ohm}$ impedance. Each input channel has overload detection before both the analog and digital anti-alias filters. Setup allows per channel selection of input voltage range $(0.1 \mathrm{~V}$, $0.3 \mathrm{~V}, 1 \mathrm{~V}, 3 \mathrm{~V}, 10 \mathrm{~V})$, transducer sensitivity, sensor type (e.g., acceleration, force, pressure, etc.), and coupling selections for $\mathrm{DC}, \mathrm{AC}$ (high pass cutoff at $0.7 \mathrm{~Hz}, 3 \mathrm{~Hz}$ or $22 \mathrm{~Hz}$ ), ICP and TEDS

Differential amplifier, programmable gain amplifier, antialiasing filters, and 24-bit Analog to Digital Converter (ADC).

An analog filter plus a $160 \mathrm{~dB} /$ octave linear-phase digital filter prevent aliasing and phase distortion.

Up to $42 \mathrm{kHz}$ analysis frequency (96k samples per second).

\pm 10 Vpeak

Voltage or ICP sensor power (4.7 mA, 30 Vpeak open circuit) and TEDS

\pm 40 Veak without damage

24-bit

$120 \mathrm{dBfs} 110 \mathrm{~dB}$ minimum in FFT mode.

$\pm 0.08 \mathrm{~dB}(1 \mathrm{kHz}$ sine at full-scale)

Within $\pm 0.04 \mathrm{~dB}$

Within \pm 0.5 degree

(from DC to $42 \mathrm{kHz}$, frequency response measurement, both inputs on the same input range, linear average)

$>117 \mathrm{~dB}$ stopband rejection

$>100 \mathrm{~dB}$ (from DC to $1000 \mathrm{~Hz}$ measured with half-fullscale sine wave).

$<-110 \mathrm{~dB}$

$<-105 \mathrm{dBfs}$

Within $0.01 \%$

Waveform Source with 2 output channels.

24-bit Digital to Analog Converter (DAC), with analog and digital anti-imaging filters.

A $160 \mathrm{~dB} /$ octave digital filter plus an analog filter prevent imaging and phase distortion.

Up to $42 \mathrm{kHz}$ output frequency (96k samples per second)

\pm 10 Vpeak

24-bit

$120 \mathrm{dBfs}$

$<-95 \mathrm{dBfs}$

$50 \Omega$

$500 \mathrm{~mA}$ peak

\section{Software}

Operating system Architecture

Applications

\section{Features}

PC configuration

PC expansion

\section{General}

Power

9 to 36 Volts DC

Dimensions

Height

Width

Depth

Weight

Temperature

Humidity requirements.
Windows XP/2000/Me/98

Distributed processing relieves the PC from the burden of realtime processing. True multitasking allows the PC to deliver maximum graphics performance and responsiveness to the user. The software provides both on-line test status and management through text displays, software toggle buttons, and screen displays of multiple time and/or frequency signals.

- Signal Analysis and Waveform Source

- Modal Data Acquisition

- Acoustic Analysis

- Rotating Machinery Analysis

- Transient Capture and SRS Analysis

- Automatic Pass/Fail Testing

- Waveform Recording

- Data Recording

On-line help, consistent management of user defined engineering units, on-line graphics, and test documentation of both measurement parameters and signals through Microsoft Word as printed media or disk files via single click on Icon.

Aluminum and steel case encloses low-noise input/output boards with dual $75 \mathrm{MHz}$ 32-bit floating-point DSP processors per board. Rubber corner guards for shock protection and stabilization. Front panel - BNC connectors for inputs and outputs, 2 color status LEDs. Rear panel - sync connector

From four up to twenty total analog inputs. Expansion by four-input modules.

PC with USB connector. Windows XP/2000/Me/98 Operating System, and Microsoft Word are the only interrupt data acquisition and realtime processing.

$4.5 \mathrm{in.} \quad 11.4 \mathrm{~cm}$

$11.25 \mathrm{in.} \quad 28.6 \mathrm{~cm}$

$10.5 \mathrm{in.} \quad 26.7 \mathrm{~cm}$

$13.5 \mathrm{lbs} \quad 5.85 \mathrm{~kg}$

32 to $122^{\circ} \mathrm{F} \quad 0$ to $50^{\circ} \mathrm{C}$

$10 \%$ to $90 \% \mathrm{RH}$ non-condensing 


\section{RT Pro Signal Analysis and Waveform Source Software}

RT Pro offers powerful data acquisition and realtime signal processing capabilities. "On the fly" changes to the measurement setup allows you maximum flexibility and gives results fast. The Signal Analysis and Waveform Source base package of RT Pro provides comprehensive capabilities for general signal analysis. Optional software packages in the RT Pro Dynamic Signal Analysis Series offer many more application tailored solutions.

\section{Signal Processing Functions}

Time domain

Time capture, auto-correlation and cross-correlation functions, orbit plots, and statistics.

Frequency domain Realtime spectrum analysis, auto-power spectrum, crosspower spectrum, power spectral density, frequency response function, coherence function, Fourier transforms, impulse responses, and ceptstrum.

Amplitude domain Histogram

\section{Realtime Spectrum Analysis}

Realtime rate Dynamic range Frequency range

Zoom

Resolution

Windows

$42 \mathrm{kHz}$ for tri-spectrum analysis with 20 inputs

$120 \mathrm{dBfs}$

$\mathrm{DC}$ to $42 \mathrm{kHz}$ in thirty-six ranges.

Thirty-two spans from $17 \mathrm{~Hz}$ to $10.5 \mathrm{kHz}$; max. upper frequency of $42 \mathrm{kHz}$.

$110,225,450,900,1800$ or 3600 spectral lines*

Hanning, Hamming, Flat-Top, Uniform, Force/Exponential,

Kaiser Bessel, Blackman, Blackman Max. Decay,

Blackman Min. Sidelobe, Bartlett, Tukey and Welch.

\section{Averaging}

Modes

Types

Overlap processing

No. of averages

Frame reject

\section{Time or Frequency}

Exponential, linear, peak hold, peak hold for specified number of averages.

User-defined percentage from $0 \%$ to $99 \%$. Maximum overlap dependent on sampling rate.

1 to 32767 frames

Automatic reject of frames with voltage overloads: manual accept/reject of overloaded frames; manual accept/reject for all frames.

\section{Triggering}

Source

Slope

Level

Pre/post-trigger

Modes

Run modes

Input channel, waveform source signal, digital input, time delay, or free run.

Positive, negative or bi-polar

Percent of full-scale range or voltage level

User selected number of samples; up to the selected frame size before or up to 65535 samples after the trigger point

Automatic or manual

Trigger first frame followed by free run, auto trigger every frame, manual arm every frame.

\section{Transient Capture}

Sampling rates Frame size

Up to 48000 samples per second in twenty-two settings. 256, 512, 1024, 2048, 4096 or 8192 samples*. Deep memory capture adds frame sizes of 16384, 32678, 65536,131072 and 262144

Modes Single frame, multiple frames

\section{Waveform Source}

Signals

Swept-sine, shaped random, shaped burst random, white noise, pseudo-random, burst random, burst chirp, chirp, sine wave, square wave, and triangle wave, impulse chain, arbitrary waveform, and DC level.

* UP to 1800 lines with all functions enabled for all channels. Higher resolutions dependent on the number of functions and channels active.

\section{Measurement Controls}

Measurement and Source Panel toggle buttons and tool bar icons provide easy access to test controls. For added convenience, commonly used commands are accessible via keyboard special function keys.

Controls

Requests

Parameters

Waveform source

Icons

Status displays

\section{Signal Displays}

Unlimited number of display windows in tile or cascade format with click \& drag zoom, user annotation, and cursors.

Window format

Per window choice of single, dual, or four pane formats Each pane can display single or multiple signals overlaid in either time or frequency. Independent choice of color and texture for signals, grids, tick marks, labels, titles, etc.

Scale format

Cursors Linear or logarithmic scales for $X$ and $Y$ axes with automatic or manual scaling.

Single or dual with $\mathrm{X}, \mathrm{Y}, \Delta \mathrm{X}, \Delta \mathrm{Y}, \Delta \mathrm{RMS}$ and $\mathrm{Q}$ value readouts; manual peak marks; automatic peak/valley detection and marks; harmonic and sideband cursor.

Frequency signals Auto-spectrum, cross-spectrum, FFT, power spectrum density, frequency response function, coherence.

Signal formats Bode, magnitude, phase, unwrapped phase, polar, vector (Nyquist), real, imaginary.

Engineering units English/SI/Metric/mixed units for acceleration, velocity, displacement, force, and pressure; user defined.

Normalization Engineering Units (EU), EUpeak, EUrms, EU², EU²/Hz, $\mathrm{EU} / \sqrt{ } \mathrm{Hz}, \mathrm{EU}^{2}-\mathrm{S} / \mathrm{Hz}$; decibels $(\mathrm{dB})$

Frequncy axis Time signals

Amplitude signals

Statistics

$\mathrm{Hz}$ or CPM

Input time histories, auto and cross correlation, and orbit plots.

Histograms

Strip chart output for rms, mean, peak, max and min values of input signals

\section{Signal Calculator}

This feature allows you to create customized signals. All signals are calculated and displayed "live" during testing. Operations include add/subtract/multiply/divide and single/double integration or differentiation, $A, B, C$ weighting, square and square root, mobility and admittance.

\section{Data Export}

RT Pro provides seamless data interfaces to advanced analysis packages.

Binary file formats Dactron, ME Scope, MATLAB, UFF, WAV, Agilent SDF, ASCII file formats $\quad$ UFF, $X-Y$ pair, $Y$ only

\section{Active X Signal Reader}

Active $\mathrm{X}$ API provide access to Dactron binary data files through programs such as Matlab, Labview, Visual Basic, Visual C, etc.

\section{Post-Test Documentation}

Icon for single click generation of data plots and test reports, including measurement parameter listings, test logs, and formatted signal plots, within Microsoft Word. Optional provision for saving documents in Adobe Acrobat PDF format. 


\section{RT Pro Dynamic Signal Analysis Series}

The RT Pro Dynamic Signal Analysis Series offers application software options to meet tomorrow's needs for noise and vibration analysis. As your test demands grow you can add new RT Pro applications to extend the usefulness and power of your system.

\section{Modal Data Acquisition}

(included with RT Pro Signal Analysis and Waveform Source)

FRF \& Coherence Arbitrary assignment of response-excitation pairs from $\begin{array}{ll}\text { Spectrum } & \text { among available inputs. H1 and H2 FRF calculations } \\ & \text { Auto-spectra only or auto-spectra and cross-spectra }\end{array}$ Time Capture $\quad$ Frame size up to 262,144 (using deep memory capture)

Special windows Force/exponential window with user-set start point, flat top points, and damping factor.

Modal coordinates Entry of measurement point, axis and sense in Channel Parameters table or via On-line Coordinate Update table.

Auto-incrementing Automatic updating of roving measurements using pre-set measurement point increment.

Frame reject Automatic reject of frames with voltage overloads: manual accept/reject of overloaded frames; manual accept/reject for all frames.

Modal package Data interface for popular modal analysis packages.

interface

\section{Long Waveform Recorder Option}

This software option enables streaming of long data records. Each record contains gap-free data simultaneously sampled for all active channels. Note that real time signal analysis can be performed during waveform recording.

\section{Max Rate $\quad 96 \mathrm{k}$ samples per second per 20 channel $^{*}$ \\ Data formats Dactron binary, X-Y ASCII, Y-only ASCII, UFF binary, \\ $\begin{array}{ll}\text { UFF ASCII, WAV, Agilent SDF, MTS ATI/AFU } \\ \text { On-line displays } & \text { Input time histories for all inputs; channel status including }\end{array}$ voltage levels and overloads. FFT, autospectra, cross spectra, FRF, coherence statistics, and waterfall displays are also possible \\ Post-processing Via playback in Analyze Anywhere option}

* Maximum throughput rate may be limited by the hard disk access time or CPU loading.

\section{Data Recorder Option}

The Data Recorder option provides a friendly tape recorder user interface for easy and quick data recording for all active channels and includes voice channel annotation via the PC sound card. Data Recorder also provides a quick and seamless transition to data playback and processing via the Analyze Anywhere option. Note that real time analysis cannot be done while using the Data Recorder.

\section{Max Rate \\ Data Formats \\ On-line displays \\ Post-processing \\ Voice Recording \\ 96k samples per second with 20 channels \\ Dactron binary, X-Y ASCII, Y-only ASCII, UFF binary, UFF ASCII, WAV, Agilent SDF, MTS ATI/AFU \\ Input time histories for all inputs; channel status; recording view with summary of index files, recording events and voice records \\ Via playback in Analyze Anywhere option \\ Unlimited number of voice recordings; each voice record up to 10 seconds}

\section{Analyze Anywhere Option}

Display and review data at your desk by using Analyze Anywhere ${ }^{\mathrm{TM}}$. This software can reside on any PC and does not require any Dactron hardware. It offers all of the on-line display features and report generation features available with a Dactron System. Data is easily imported via disk media or across the network.

Data playback allows you to process throughput data collected with Long Waveform Recorder. You can also process data from other data acquisitions systems that export data in either X-Y ASCII, Y-only ASCII, UFF ASCII or UFF Binary format. All of the RT Pro signal processing functions are available for data playback.

\section{Environmental Data Reduction Option \\ Transient Capture}

Sampling rates

Up to 48000 sps in twenty-two settings.

Frame size

$256,512,1024,2048,4096$ or 8192 samples*

Modes

Single frame, multiple frames

Averaging

Exponential, linear, peak hold, peak hold for specified number of averages

\section{Shock Response Spectrum}

SRS analysis

Up to 14 octave range using maxi-max, negative maximum, and positive maximum analysis techniques. User specifies high and low frequency, reference frequency, damping ratio or $\mathrm{Q}$ value, and resolution (1/1, $1 / 3,1 / 6,1 / 12,1 / 24$, or $1 / 48$ )

\section{Automated Test Options}

\section{Pass/Fail Limits and Criteria}

Limits checking

Limits definition

Multiple, simultaneous limit checks on frequency domain, time domain, and amplitude domain signals.

Pass/fail limit criteria may be defined based on:

- User created Limit Tables

- Measured signals resident in memory

- Signals imported from ASCII files

- Synthesized signals generated by using RT Pro's Signal Calculator function

Limit tables

High or low limit curves defined based on breakpoint table; interpolation on linear-linear, log-linear, linear-log, or log-log basis.

Limits import

Limit tables seeded from imported ASCII, UFF or Dactron binary file. ASCll files generated by spreadsheets, MATLAB, or other software.

Limit Scaling

Limit check range

Limit curve scaling by using a user entered value as a multiplying constant or offset value.

Sample by sample checking (time domain) or line by line checking (frequency domain); check range may be the whole range or a user specified range.

Limit threshold

User specified percentage of values outside of limits to trigger fail flag.

Overall limits

Fail detection based on the RMS, maximum, mean minimum, or peak value. (Time and Amplitude Domain only).

Actions on fail flag Display alarm message, sound PC beep, generate test report, and abort measurement. Frequency domain User message strings displayed on test failure.

Auto and cross spectra, $1 / 1$ and $1 / 3$ realtime octave spectra1, Frequency Response Function and coherence, and Shock Response Spectrum (SRS) ${ }^{2}$.

Time domain Time histories, synchronously averaged time records, and auto and cross correlations (overall value limit checking only for correlations).

Amplitude domain
User messages

\section{Test Schedule}

User defined sequence of events that are automatically executed during the test.

Events

Measurement duration (hours, minutes, seconds), limit checking on or off, start or stop the source signal, timed pause, save signals, and generate a test report; logic for sequence loop and nested loops. 


\section{RT Pro Dynamic Signal Analysis Series}

The Acoustic Analysis and Rotating Machinery Analysis options offer advanced on-line processing capabilities. The Acoustic Analysis option provides realtime time-domain octave filtering for $1 / 1$ and $1 / 3$ octave spectra. The Advanced Graphics and RPM Spectrum Processing options provide rpm-based waterfalls, spectrograms, and color contour plots. The Realtime Order Tracking option allows for order analysis of up to 55 orders simultaneously.

\section{Acoustic Analysis Option}

\section{Realtime Octave Analysis}

Method

Standards

1/1 octave bands Conform to ANSI Standard S1.11-1986, Order 7, Type 1-

D, Extended and Optional Frequency Ranges

1/3 octave bands Conform to ANSI Standard S1.11-1986, Order 3, Type 1D, Extended and Optional Frequency Ranges

Frequency ranges

Weighting

Averaging modes

Sound level

detectors

Measurement

period

FFT auto-spectra

Averaging

Resolution

Windows

Frequency signals Barchart display

Time signals

\section{$1 \frac{20 \text { inputs }}{1 \mathrm{~Hz}-16 \mathrm{kHz}}$ \\ $1 \mathrm{~Hz}-20 \mathrm{kHz}$}

Linear, A, B and C selectable

Linear, exponential or peak hold

Peak hold, impulse, fast and slow sound level measurements

From $1.3 \mathrm{msec}$ to 48 hours

Simultaneously measured during realtime octave acquisition.

None, exponential, linear, or peak hold

225,450 or 900 spectral lines

Hanning, Hamming, Flat-Top, Uniform, Kaiser Bessel, Blackman, Blackman Max. Decay, Blackman Min. Sidelobe, Bartlett, Tukey and Welch.

$1 / 1$ and $1 / 3$ octave spectra and auto-spectra

Solid or transparent with multiple signal overlays.

Input time histories, overall level (linear or A weighted) versus time, user-selected octave band level versus time.

\section{Advanced Graphics Option}

(included with RT Pro Signal Analysis \& Waveform Source, Order Tracking and Acoustic Analysis Options)

\begin{tabular}{|c|c|}
\hline $\begin{array}{l}\text { Quantities } \\
\text { Plot formats }\end{array}$ & $\begin{array}{l}\text { Spectra and time histories versus time } \\
\text { Waterfall ( } 3 \text {-D display), waterfall with single pane or dual } \\
\text { pane, and spectrograms or color contour (2-D display) } \\
\text { plots }\end{array}$ \\
\hline \multicolumn{2}{|c|}{ W ater fall An alysis } \\
\hline $\begin{array}{l}\text { Cursors } \\
\text { 3-D Cursor } \\
\text { Synching }\end{array}$ & $\begin{array}{l}\text { Dual axis cursor with trace color highlighted in both axes. } \\
\text { Synchronized cursor positioning for all cursors in all } \\
\text { windows. }\end{array}$ \\
\hline $\begin{array}{l}\text { Display axes } \\
\mathbf{X} \text { axis } \\
\mathrm{Y} \text { axis }\end{array}$ & $\begin{array}{l}\text { Hertz or CPM; linear or log scale } \\
\text { Engineering Units (EU), EU peak, EUrms, EU }{ }^{2}, \quad \mathrm{EU}^{2} / \mathrm{Hz}, \\
\text { EU } / \sqrt{ } \mathrm{Hz}, \mathrm{EU} 2-\mathrm{S} / \mathrm{Hz} \text {; linear, log or } \mathrm{dB} \text {. }\end{array}$ \\
\hline $\begin{array}{l}Z \text { axis } \\
\text { 3-D orientation } \\
\text { Slice plot }\end{array}$ & $\begin{array}{l}\text { Seconds } \\
\text { Viewing angle interactively set by using the mouse } \\
\text { Selectable as X slice, Z slice }\end{array}$ \\
\hline
\end{tabular}

\section{RPM Spectrum Processing Option Tachometer \\ Pulses per rev. \\ Gear ratio \\ RPM range \\ RPM accuracy \\ Level range \\ 1 to 1024 \\ Ratio of two numbers; each from 0.1 to 10000 \\ $1<$ RPM $<300000$ \\ 100 ppm (typical) \\ Programmable from $1 \mathrm{~V}$ to $10 \mathrm{~V}$}

\section{RPM Trigger \\ Level \\ Slope}

RPM plus tolerance setting

Run-up, run-down, absolute value

\section{Run Modes}

RPM Waterfall

Frame size

Spectral lines

Averaging

Overlap

Windowing

Waterfall plots

Low RPM, high RPM, and delta RPM

256, 512, 1024, 2048 or 4096 samples*

$110,225,450,900$ or 1800 lines*

Stable (linear), peak hold, or exponential

User specified from 0 to $99 \%$; maximum overlap dependent on sampling rate.

Hanning, Hamming, Flat-Top, Uniform, Bartlett, Tukey, Blackman, Blackman (4th) Maximum, Blackman (4th) Minimum, and Welch. attributes as per the Advanced Graphics Option.

\section{Real Time Order Tracking Option}

(Includes the Advanced Graphics and RPM Spectrum Processing Options.)

\begin{tabular}{|c|c|c|}
\hline \multirow[t]{2}{*}{$\begin{array}{l}\text { Method } 1 \\
\text { Order span } \\
\text { Order resolution }\end{array}$} & \multicolumn{2}{|c|}{$\begin{array}{l}\text { Realtime digital resampling technique } \\
1 \text { st up to } 320 \text { th order tracked; } 1<\mathrm{RPM}<300000 \\
0.025,0.05,0.1,0.125,0.25,0.5 \text { and } 1.0 \\
\text { Max Order Order Resolution }\end{array}$} \\
\hline & $\begin{array}{l}20 \\
40 \\
80 \\
160 \\
320\end{array}$ & $\begin{array}{l}0.025 \text { to } 1 \\
0.05 \text { to } 1 \\
0.1 \text { to } 1 \\
0.125 \text { to } 1 \\
0.25 \text { to } 1\end{array}$ \\
\hline
\end{tabular}

Number of orders Up to 55 orders simultaneously tracked online.

Amplitude Based on DFT frequency domain extraction of order extraction amplitudes.

Run Mode $\quad$ Run-up, Run-down, and Free run. Selectable number of Runs with automatic rejection of data that violates the Run mode criterion (wrong RPM direction)

Waterfall plots Amplitude vs Order vs RPM; all other attributes as per the Advanced Graphics Option.

Method 2

Order span FFT based amplitude detection

Order resolution

1 st up to 55 th order tracked; $1<$ RPM $<300000$

0.1 to 200th order in post-process mode.

(Maximum useable RPM limited by resolution, tach pulse rate, pulses/rev and averaging used)

Number of orders Up to 55 orders simultaneously tracked online; unlimited orders in post-process mode.

Amplitude

extraction

Selectable based on fixed bandwidth, fixed spectral lines, or frequency rang; additionally, proportional bandwidth (from $1 \%$ to $100 \%$ ) in post process mode.

Waterfall plots

* Maximum resolution depends on the number waterfall spectra, order tracks defined, and channels active. Up to 3600 lines with two channels, 75 spectra per waterfall, and 20 order tracks per channel. 


\title{
RT Pro Dynamic Signal Analysis Series
}

\begin{abstract}
Dactron's Rotate and Rotate Plus options offer advanced and robust post-processing for diagnosing and analyzing noise and vibration problems due to periodic loading from engines, transmissions, drive lines, wheels, belt drives, bearings, turbines, or reciprocating machines such as compressors. All results, both graphics and numeric data, are easily exported to Mircosoft Excel, Word or PowerPoint
\end{abstract}

\section{Dactron Rotate Option}

Dactron Rotate's fast computations with waterfall and color contour plots make it easy to quickly identify trouble areas while the precision of its computed order tracking and flexible cursors help to immediately pinpoint root causes.

\section{Tachometer Analysis}

Tachometer Analysis computes raw or initial estimates of a machine's instantaneous rotating speed from sampled data from a DC or Pulse tachometer signal. A smoothing function fits cubic spline segments to the raw estimate to give a speed curve that is free of tachometer noise and drop out effects.

\begin{tabular}{|c|c|}
\hline $\begin{array}{l}\text { Tach type } \\
\text { Tach conversions } \\
\text { Pulse signal } \\
\text { DC signal }\end{array}$ & $\begin{array}{l}\text { Pulse or DC signal } \\
\text { User specified pulses per revolution } \\
\text { User specified RPM per Volt and RPM at zero Volts }\end{array}$ \\
\hline $\begin{array}{l}\text { Triggering } \\
\text { Slope } \\
\text { Level } \\
\text { Hysteresis } \\
\text { Hold off }\end{array}$ & $\begin{array}{l}\text { Positive or negative } \\
0 \text { to } 10 \text { Volts } \\
0.01 \text { to } 10.0 \\
\text { Programmable minimum time between trigger events }\end{array}$ \\
\hline
\end{tabular}

Smoothed RPM Cubic spline segments provide smoothed estimate of the machine's rotating speed. A unique algorithm removes "outliers" from the raw rpm curve estimate and then recomputes the spline fit. This algorithm makes it possible to generate good speed curve estimates even when using a noisy tachometer signal with pulse dropouts.

Spline segments $\quad 1$ to 99

Shave \% $0 \%$ to $20 \%$

\section{Computed Order Track Analysis}

Order Tracking digitally resamples based on the smoothed machine speed curve providing alias-free order tracks of amplitude and phase versus RPM.

Order numbers Simultaneous calculation of multiple orders; fractional or Tracking Method

Type integer orders

RPM, Time or None

Range and Run Down or Run Up

resolution

User specified Min. and Max. RPM or Sec., RPM or Sec. increment, and Max. number of values

Signal Processing

Filter width $1 \%$ to $100 \%$

Weighting Selectable A, B or C

Signal domain Single or double Integration/ Differentiation

\section{Torsional Analysis}

Based on pulse signals from encoders and flywheels, Torsional Analysis gives an accurate kinematic description of torsional vibrations from single station measurements. This technique processes time domain data, using even relatively coarse measurements, to produce a torsional signature which then is used to make waterfall and order calculations and graphics.

\section{Waterfall Analysis}

Waterfall Analysis uses fast Fourier transforms to calculate an array of FFT spectra. Flexible parameter settings and fast computation and display times provide waterfall plots and color contour displays (spectrograms) for the computed FFT arrays.

\begin{tabular}{|c|c|}
\hline $\begin{array}{l}\text { Quantities } \\
\text { Frame size } \\
\text { Spectral lines }\end{array}$ & $\begin{array}{l}\text { Power Spectral Density (PSD) or Cepstrum } \\
128,256,512,1024,2048,4096,8192,16384 \text { or } 32768 \\
\text { samples } \\
50,100,200,400,800,1600,3200,6400 \text { or } 12800 \text { lines }\end{array}$ \\
\hline $\begin{array}{l}\text { Averaging } \\
\text { Number } \\
\text { Type } \\
\text { Overlap } \\
\text { Windowing } \\
\text { Type } \\
\text { Correction } \\
\text { Weighting } \\
\text { Signal domain } \\
\text { Tracking } \\
\text { Method } \\
\text { Type } \\
\text { Range and } \\
\text { resolution } \\
\text { Cursors } \\
\text { Type } \\
\text { Axis orientation } \\
\text { Display axes } \\
\text { X axis } \\
\text { Y axis } \\
\text { Z axis } \\
\text { 3-D orientation }\end{array}$ & $\begin{array}{l}\text { Selectable from } 1 \text { to } 500 \\
\text { Stable (linear), peak hold, or exponential } \\
\text { User specified from } 0 \text { to } 50 \% \\
\text { Hanning, Hamming, Flat Top, or Uniform } \\
\text { Narrowband (peak) or Wideband (rms) } \\
\text { Selectable A, B or C weighting } \\
\text { Single or double Integration/ Differentiation } \\
\text { RPM, time or none } \\
\text { Run Down or Run Up } \\
\text { User specified Min. and Max. RPM or Sec., RPM or Sec. } \\
\text { increment, and Max. number of spectra } \\
\text { Single, band or harmonic } \\
\text { Constant frequency, order, RPM or time } \\
\text { Hertz or orders; linear or log scale } \\
\text { Linear, log or dB scale } \\
\text { RPM, seconds or number } \\
\text { Viewing angle interactively set by using the mouse }\end{array}$ \\
\hline
\end{tabular}

\section{Dactron Rotate Plus Option}

Rotate Plus provides added features for dealing with problems associated with families of harmonics. Re-sampling in the angle domain isolates information vital to diagnosing harmonic problems found in transmissions, bearing and gears.

\section{Millstrum Analysis}

This analysis is a variation on Cepstrum analysis and it helps you to identify families of harmonics present in your data. This analysis is particularly powerful in picking out families of harmonics that are obscured by noise or harmonics dominated by the amplitudes of unrelated orders.

\section{Order Normalized Spectra}

By resampling data in the angle domain, better order tracking resolution is obtained for data with a large dynamic range and in lower frequency ranges. Resampling in the angle domain at constant intervals maintains the same angle resolution around the governing shaft and gives the same data quality regardless of the shaft speed.

\section{Sideband Cursors}

By overlaying Sideband Cursors on your spectra data you can easily identify families of harmonics embedded in your data.

\section{Bearing Cursors}

These cursors highlight specific bearing problems such as FTF (Fundamental Train Frequency), BSF (Ball Spin Frequency), BPIR (Ball Pass Inner Race), BPOR (Ball Pass Outer Race), and 2xBSF. 


\section{FOCUS II}

Technical Specifications

UK

USA

FRANCE

GERMANY

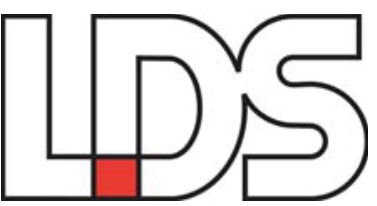

wuw.lds-cjroup.com

AN SPX COMPANY
Tel: +44 (0) 1763242424

Tel: +1 2032657966

Tel: +33 (0) 169112130

Send all E-mail inquiries to:

sales@Ids-group.com 


\section{APPENDIX G: TEST PROCEDURE}

1. Acquire required instrumentation
a. ICP Signal Conditioner with at least 3 channels
b. DAQ with sampling frequency of at least $16 \mathrm{kHz}$
c. High Speed Camera

2. Connect instrumentation to drop tower
a. Accelerometer cables to signal conditioner
b. Signal conditioner to DAQ

3. Apply power to drop tower by turning green power switch on top of control box

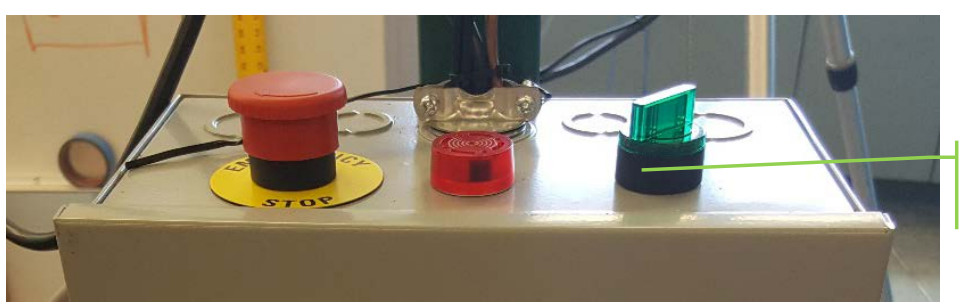

4. Apply power to instrumentation
a. Signal conditioner
b. DAQ
c. High Speed Camera
d. Laptops 
5. Secure Helmet to headform with chinstrap or duct tape

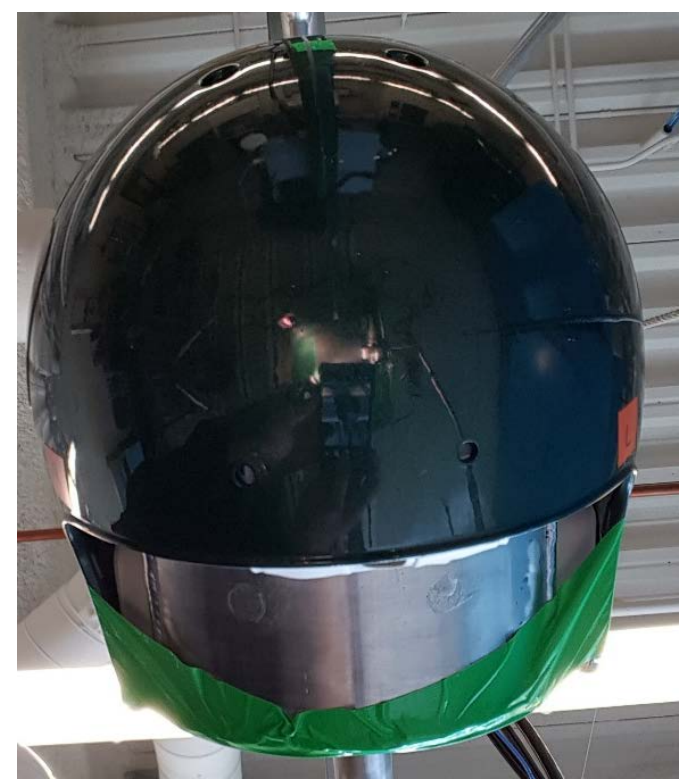

6. Adjust upper limit switch to correct height for required drop height

7. Lower lift bar to capture carriage by turning winch control switch to down

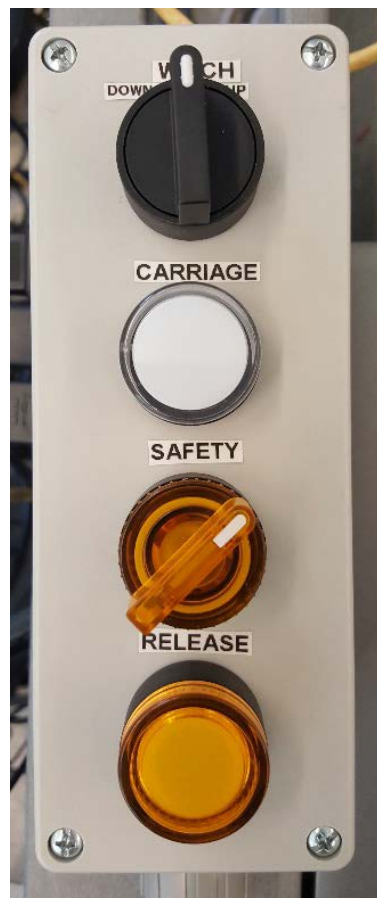

position 
8. Insert safety pin through safety bracket and lift bar

9. Raise lift bar till limit switch activated using winch control switch

10. Measure helmet to impact pad height to ensure correct height is achieved

11. Remove safety pin

12. Retreat safe distance and clear area of unnecessary personnel

13. Pretrigger high speed camera in Phantom Control Software.

14. Start DAQ data recording on DAQ software of choice

15. Turn Safety Switch

16. Release carriage

17. When impact is heard, trigger high speed camera in software

18. Stop DAQ recording

19. Save data and video

20. Readjust helmet

21. Repeat 5-19

22. When testing completed, remove power from all instrumentation and drop tower 
X Axis:

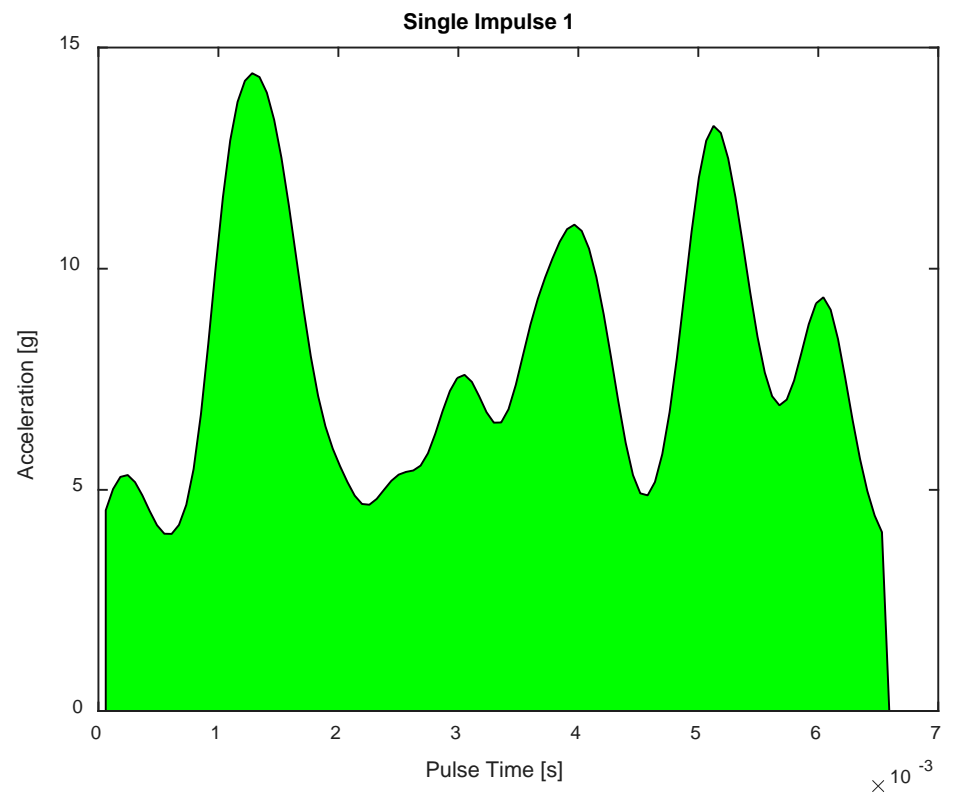

Y Axis:

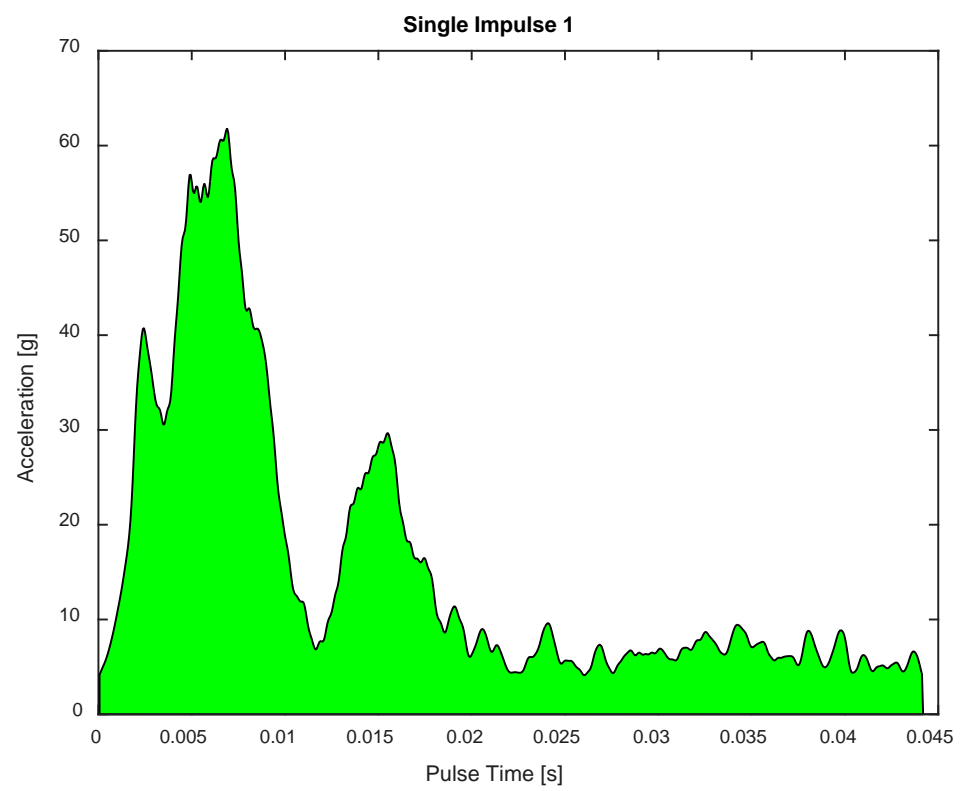


Z Axis:

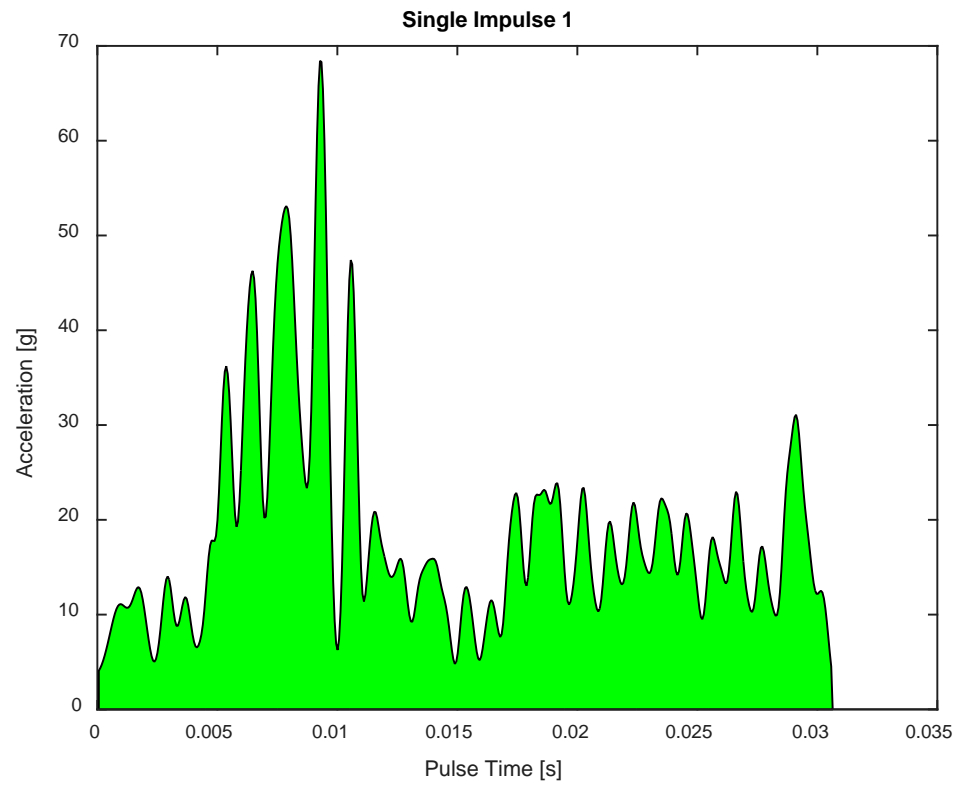

